\title{
Stress and Deformation in Orogenic Wedges with Power-Law Rheology
}

\author{
by \\ Jiyang Liu, B.Sc. (Hons.), M.Sc. \\ A thesis submitted to the Faculty of Graduate Studies and Research \\ in partial fulfillment of the requirements for the \\ degree of Doctor of Philosophy
}

Department of Earth Sciences

Carleton University

Ottawa, Ontario, Canada

March, 1996

(C) Copyright

1996, Jiyang Liu 
National Library

of Canada

Acquisitions and

Bibliographic Services Branch

395 Wellington Street

Ottawa, Ontario

K1A ON4
Bibliothèque nationale

du Canada

Direction des acquisitions et

des services bibliographiques

395, rue Wellington

Ottawa (Ontario)

K1A ON4
The author has granted an irrevocable non-exclusive licence allowing the National Library of Canada to reproduce, loan, distribute or sell copies of his/her thesis by any means and in any form or format, making this thesis available to interested persons.
L'auteur a accordé une licence irrévocable et non exclusive permettant à la Bibliothèque nationale du Canada de reproduire, prêter, distribuer ou vendre des copies de sa thèse de quelque manière et sous quelque forme que ce soit pour mettre des exemplaires de cette thèse à la disposition des personnes intéressées.

L'auteur conserve la propriété du droit d'auteur qui protège sa thèse. Ni la thèse ni des extraits substantiels de celle-ci ne doivent être imprimés ou autrement reproduits sans son autorisation. 
Dissertation Abstracts International is arranged by broad, general subject categories. Please select the one subject which most nearly describes the content of your dissertation. Enter the corresponding four-digit code in the spaces provided.

$$
\text { Geophysics }
$$

\section{Subject Categories}

\section{THE HUMANITIES AND SOCIAL SCIENCES}

\section{COMMUNICATONS AND THE ARTS}

Architecture

Ant History ................................. 0729

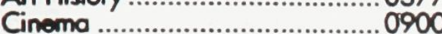

Dance ........................ 0378

Fine Arts ....................................... 0357

Information Science ................... 0723

Journalism ............................... 0391

Library Science ..................... 039

Mass Communications ................ 0708

Music ....................................... 0413

Speech Communication .................. 0459

\section{EDUCATIN}

General ....................................0515

Administration .................................0514

Adult and Continuing ................. 0516

Agricultural ............................. 0517

Bilingual and Multicultural......

Business

Curriculum and Instruction ............ 0727

Earty Childhood ........................ 0518

Elementary ............................. 0524

Finance ............................... 0277

Guidance and Counseling .......... 0519

Healith ....................................... 0680

Higher ................................... 0745

Hemes Economics

Language and Literature ................ 0279

Mathematics ............................ 0280

Music ................................. 0522

Physical ........................... 0523
Theoter ...................................... 0465

Community College ....................... 0275

Industrial .............................. 0521

Philosophy of ......................... 0998

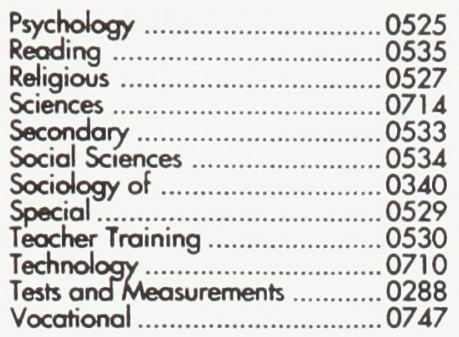

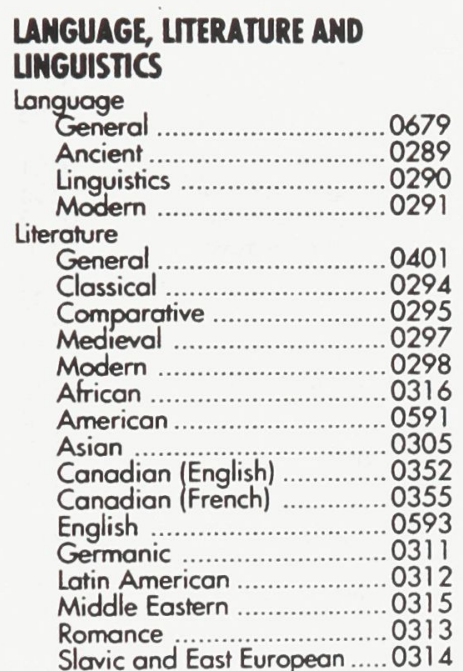

\section{THE SCIENCES AND ENGINEERING}

\section{BIOLOGICAL SCIENCES}

Agriculture

General ............................ 0473

Agronomy ........................... 0285

Animal Culture and

Nutrition ......................... 0475

Animal Pathology ................. 0476

Food Science and

Technology ...................... 035

Forestry and wildalife ..............0478

Plant Culture ........................ 0479

Plant Pathology .................... 0480

Plant Physiology .................. 0817

Range Management ............. 0777

Biology

General ............................. 0306

Anatomy .......................... 028

Biostatistics ........................ 0308

Botany ............................ 0309

Cell Ecology ................................. 0329

Entomology ....................... 0353

.

Limnology .......................... 0410

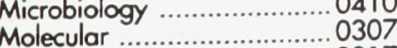

Neuroscience ...................... 0317

Oceanography ................... 0416

Physiology ..........................0433

Radiation

Veterinary Science .................. 0778

Zoology ............................. 0472

Biophysics

General ........................... 0786

\section{EARTH SCIENCES}

Biogeochemistry

Geochemistry

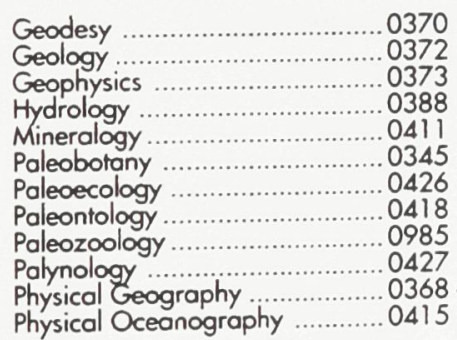

\section{HEALTH AND ENVIRONMENTAL}

\section{SCIENCES}

Enyironmental Sciences .............0768

Health Sciences

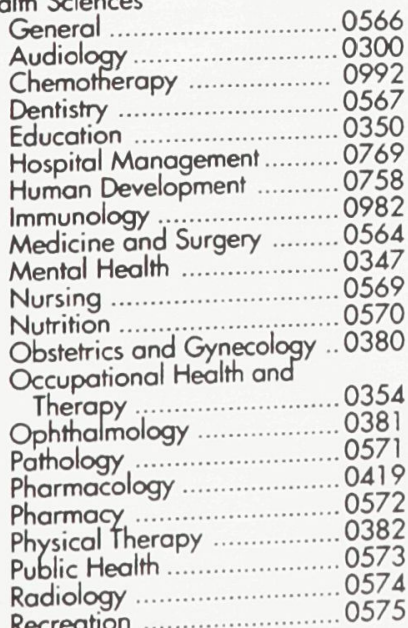

PHILOSOPHY, RELIGION AND THEOLOGY

Philosophy

Religion

0422

General

0318

Biblical Studies .................. 032

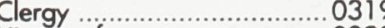

History of .......................... 0320

Philosophy of ...................... 0322

SOCIAL SCIENCES

American Studies 0323

Anthropology

Archoeology ................... 0324

Cultural ............................... 0326

Business Administration

General

0310

Banking

Management

0272

Morketing ........................... 0338

Canadian Studies

Economics

General .....

Agricultural ........................... 0503

Commerce-Business ................ 050

Finance

History

Labor

Folklore

Geography

Gerontology

General

.0508

0511

0358

0366

0351

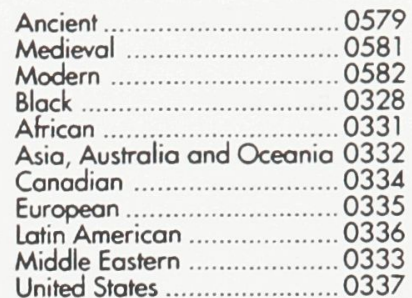

History of Science .................... 0585

Law

Political Science

General

International Low and

0615

Relations

Public Administration

Recreation

Social Work

Sociology

General .......................... 0626

Criminology and Penology ...0627

Demography 0938

Ethnic and Racial Studies ...... 0631

Individual and Family

Studies

0628

Industrial and Labor

Relations

0629

Public and Social Welfare

0630

Social Structure and

Developmen

Theory and Methods

Transportation

0709

0999

0453

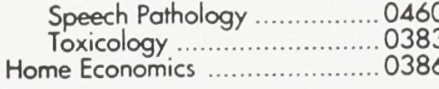

\section{PHYSICAL SCIENCES}

Pure Sciences

Chemistry

General .........

Agricultural

Anolytical

Biochemistry

Inorganic

Nuclear

Prganic.......

Physical

Polymer

Radiation

Mathematics

Physics

General .

Acoustics

Astronomy and

Astrophysics

Atmospheric Science...

Atomic

Electronics and Electricity

Elementary Particles and

High Energ

Fluid and Plasma

Molecular

Nuclear

Optic

Radiation

Solid State

Statistics

Applied Sciences

Applied Mechanics

Computer Science
Engineering

Aerospace

Agricultural

Automotive

Chemical

Civil

Electronics and Electrical

Heat and Thermodynamics Hydraulic

Industrial

Marine

Materials Science

Mechanica

Metallurgy

Mining

Nuclear

Packoging

Petroleum

Sanitary and Municipal

System Science

Geotechnology

Operations Research

Plastics Technology

Textile Technology

\section{PSYCHOLOGY}

General

Behavioral

Clinical

Developmental

Experimental

Industrial

Personality

Physiological

Psychobiology

Psychometrics

Socia

0537

0538

0540

0541

0542

0543

0544

0545

0546

0548

0743

0551

0552

0765

0554

0790

0428

0796

0994

0621

0384

0620

0623

0624

0625

0989 
Nom Dissertation Abstracts International est organisé en catégories de sujets. Veuillez s.v.p. choisir le sujet qui décrit le mieux votre
thèse et inscrivez le code numérique approprié dans l'espace réservé ci-dessous.

\section{Catégories par sujets}

\section{HUMANITES ET SCIENCES SOCIALES}

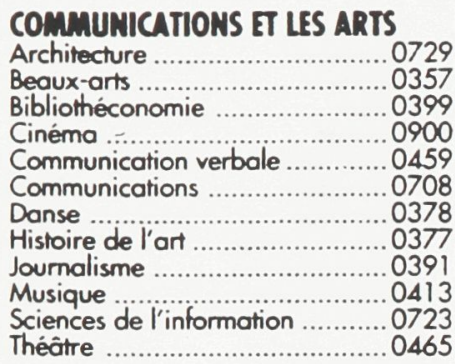

\section{EDUCATION}

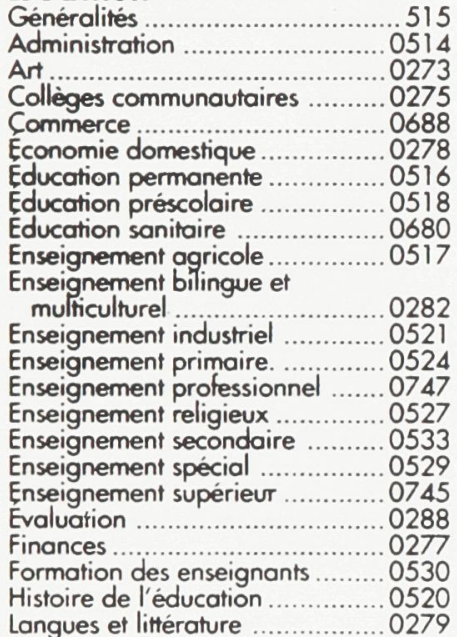

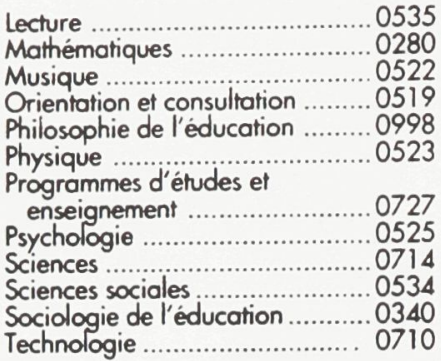

\section{LANGUE, LITTÉRATURE ET} LINGUISTIQUE

Langues

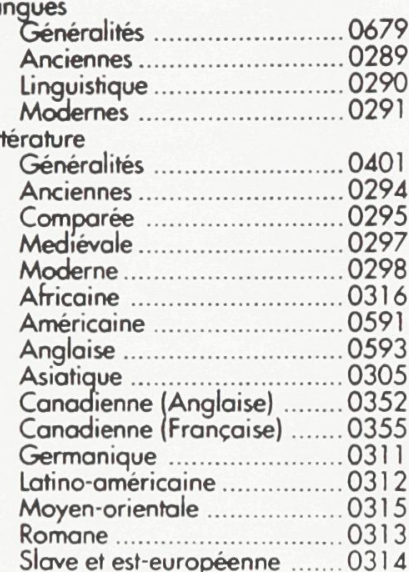

\section{PHILOSOPHIE, RELIGION ET} THEOLOGIE

Philosophie

Religion

Généralités

Clergé

Etudes bibliques

Histoire des religions

Philosophie de la religion

Théologie

\section{SCIENCES SOCIALES}

Anthropologie

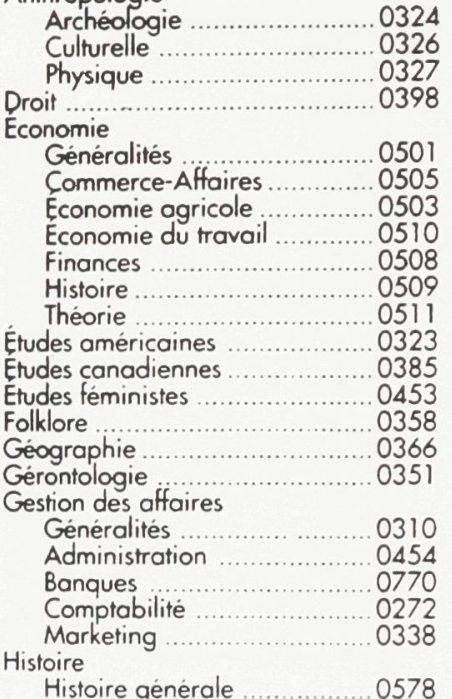

0318

0319

0321

0320

0322
0469

324

0326

398

0501

0505
503

510

509

0511
0323

0385

358

0351

0310

338

0578

\begin{tabular}{|c|c|}
\hline 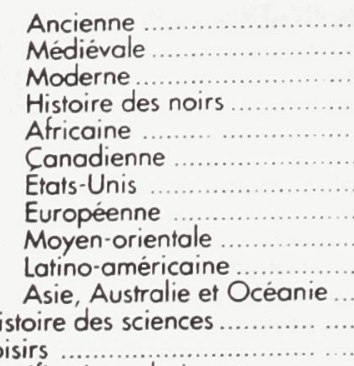 & $\begin{array}{l}058 \\
058 \\
032 \\
033 \\
033 \\
033 \\
033 \\
033 \\
033 \\
033 \\
058 \\
081\end{array}$ \\
\hline $\begin{array}{l}\text { anification urbaine et } \\
\text { régionale }\end{array}$ & \\
\hline $\begin{array}{l}\text { cience politique } \\
\text { Géneralites ............... }\end{array}$ & 0 \\
\hline $\begin{array}{l}\text { Droit et relations } \\
\text { internationales .......... } \\
\text { ciologie }\end{array}$ & \\
\hline $\begin{array}{l}\text { Généralités ...... } \\
\text { Aide et bien-atre social } \\
\text { Criminologie et }\end{array}$ & 0 \\
\hline $\begin{array}{l}\text { établissements } \\
\text { pénitentiaires .............. } \\
\text { Demographie }\end{array}$ & \\
\hline $\begin{array}{l}\text { Etudes de } \mathrm{l}^{\prime} \text { individu et } \\
\text { de la famille }\end{array}$ & .06 \\
\hline $\begin{array}{l}\text { Etudes des relations } \\
\text { interethniques et } \\
\text { des relations raciales ...... } \\
\text { Structure et développemient }\end{array}$ & \\
\hline $\begin{array}{l}\text { fructure ef developpenient } \\
\text { social } \\
\text { Thérie et méthodes. }\end{array}$ & $\begin{array}{l}.07 \\
.03\end{array}$ \\
\hline $\begin{array}{l}\text { Travail et relations } \\
\text { industrielles } \\
\text { nsponts } \\
\text { vail social }\end{array}$ & \\
\hline
\end{tabular}

\section{SCIENCES ET INGENIERIE}

\section{SCIENCES BIOLOGIOUES}

Agriculture

Généralités

Alimentation et rechnologie

alimentaire .................... 0359
Culture

Élture ......................... 0479

Exploitation des péturages ...00777

Pathologie animale .............. 0476

Pathologie végétale ............ 0480

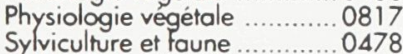

Sylviculture et foune ............ 0478
Technologie du bois ............0746

Biologie

Généralités ....................... 030

A atomie ........................0287

Biologie moléculaire ............. 0307

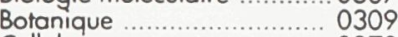

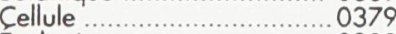

Ecologie ........................ 0329

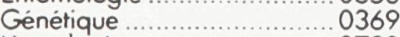

Limnologie .............................. 0793

Microbiologie ................... 0410

Neurologie …....................... 0317

Océanographie …................. 0416

Radiation .......................... 0821

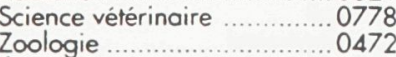

Zoologie

Genéralités

Medicale

0786

0760

\section{SCIENCES DE LA TERRE}

Biogéochimie

Geochimie...

0425
0996

Geodésie

0370

0368

\begin{tabular}{|c|c|}
\hline 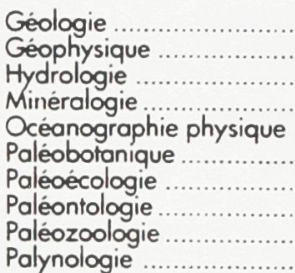 & $\begin{array}{l}.0372 \\
.0373 \\
.0388 \\
.0411 \\
.0415 \\
.0345 \\
.0426\end{array}$ \\
\hline
\end{tabular}

\section{SCIENCES DE LA SANTE ET DE}

\section{L'ENVIRONHEMENT}

Economie domestique ............... 0386

Sciences de l'environnement ....... 0768

Sciences de la santé

Généralités

Alimentation et nutrition ...0570

Audiologie

Chimiothérapie ................ 0300

Dentisterie .......................... 0567

Développement humain ........0758

Enseignement ...................... 0350

Immunologie ...................... 0982

Meisirs ................

thérapie 0354

Médecine et chirurgie ............ 0564

Obstétrique et gynécologie ... 0380

Ophtalmologie ................... 0381

Orthophonie ............................... 0460

Pathologie ........................... 0571

Pharmacie .......... 0572

Pharmacologie .......................... 0419

Physiotherapie ................... 0382

Radiologie ....................... 0574

Santé publique

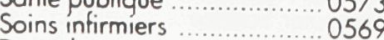

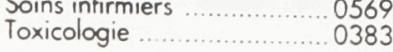

\section{SCIENCES PHYSIQUES}

Sciences Pures

Chimie

Genéralités

Biochimie

Chimie agricole

Chimie analytique

Chimie minerale

Chimie nucléaire

Chimie organique

Chimie pharmaceutique

Physique.

PolymCres

Radiation

Mathematiques

Physique

Genéralités

Acoustique.

Astronomie et

astrophysique

Electronique et électricite

Fluides et plasma

Météorologie

Optique

Particules (Physique

nucléaire)

Physique atomique

Physique de l'état solide

Physique moleculaire ....

Physique nucléaire

Radiation

Statistiques

Sciences Appliqués Et

Technologie

Informatique

génierie

Agricole

Automobile
Biomédicale

Chaleur et ther

modynamique

Condifionnemen

(Emballage)

Génie aérospatia

Génie chimiqu

Génie électronique et

électrique

Génie industrie

Génie mécanique

Génie nucléaire

Ingénierie des systämes

Meconique navale.

Métallurgie

Science des materiaux

Technique du pétrole

Technique minière

Techniques sanitaires ef

municipales

Technologie hydraulique

0607

0759

0608

0752

0798

0748

0611

0609

0610

0756

0463

Geotech appliquee

echnologie

$$
\text { (Technologie) }
$$

\section{PSYCHOLOGIE}

Generalités

Psychobiologie

0984

Psychologie clinique

Psychologie du comportement

Psychologie du développement

Psychologie expérimentale

Psychologie industrielle.

Psychologie physiologique

Psychologie sociale

Psychometrie
0541

0549

0538

0542

0546

0548

0552

0790

0547
0743

0794

0765

055

0554

0545

.0346

0795

0796
0794

0621

0625

0349

0622

0384

0623

0624

0989

0451 


\section{Abstract}

Within the framework of plate tectonic theory, orogenic belts are interpreted as forming at convergent margins as a result of subduction and collision of two plates. This thesis deals with stress and deformation in orogenic belts from two different perspectives: (1) brittle, dislocation-type propagation of thrust faults along the base of wedge-shaped elastic thrust sheets; (2) stresses and deformation of orogenic wedges with generalized nonlinear creep rheology. Both treatments are analytical and quantitative from a continuum mechanics approach.

On the scale of a single thrust sheet, an Airy stress function solution is derived for the stress field in an elastic wedge-shaped overthrust block subject to horizontal forces at the rear and at the front. From this stress solution the state of stress on the basal thrust (lower boundary of the block) is obtained. Both normal and shear stresses on the basal thrust are affected by the rear pushing and front buttressing forces and by the slopes of the upper and lower boundaries. For nonlinear components of external horizontal forces exerted on the vertical edges of the block, and/or nonzero slopes of the upper and lower boundaries, the normal and shear stresses vary systematically with position along the basal thrust. The ratio of shear to normal stresses along this plane (the "frictional function" of the thrust) can be compared with the friction coefficient to determine where slippage is likely to occur. Since the frictional function is a function of position, the strength is exceeded only on part of the thrust (near the rear for most values of the parameters). The assumption of simultaneous displacement of the whole thrust sheet on the basal plane is therefore not realistic. Even simple static models such as the present one indicate that the motion on thrust faults must be envisaged in terms of progressive, consecutive "dislocation-type" slippage. 
On a larger scale, in order to model some important features commonly associated with the development of orogenic wedges, an analytical solution for deformation and stress in a two-dimensional plane strain incompressible block with power-law bulk rheology is developed. This solution involves material rheology, stress boundary conditions at the rear of the block, and a function $C(x, y)$ representing the deviation of vertical normal stress from lithostatic overburden. If the latter is assumed to be zero, a well accepted approximation in modelling of various geodynamic processes, a simplified solution can be obtained for a linear longitudinal strain rate. This solution is expressed in terms of a group of integration constants and a one-dimensional function of depth representing the normal stress in excess of the lithostatic on the rear boundary. Both constants and function are explicitly computable from material rheology and assigned boundary conditions. Analytical comparison and numerical computations show that this linear longitudinal strain rate solution is a two-dimensional extension of a classic onedimensional solution derived by Nye (1957) for ice sheets. Also, some semi-quantitative models for orogenic wedges (e.g. Platt, 1986) can be shown to be special cases of this solution. Numerical implementation of the solution suggests that: (1) the dynamics of orogenic wedges varies with material rheology, temperature, and boundary conditions; (2) the depth distribution of the nongravitational normal stress at the rear boundary of the wedge required to maintain a prescribed deformation pattern varies mainly with wedge rheology; (3) wedges with viscoplastic rheology (i.e. a top plastic layer overlying a nonlinear viscous layer) are able to deform with different longitudinal patterns, including compression near the front and extension near the back of the wedge, if acted upon by suitable forces.

The models presented in this thesis are necessarily drastic simplifications of reality. However, they yield two results of general interest and applicability. The first is that slippage along the basal thrust of an overthrust block propagates along the fault, and is not synchronous along its whole length. The second is that orogenic wedges with linearly 
viscous, power-law viscous, and plastic rheologies can show stress and deformation patterns in reasonable agreement with observation, under tectonic forces and kinematic boundary conditions simulating a variety of tectonic styles. 


\section{Acknowledgments}

The completion of this thesis would have been impossible without the help and cooperation of various people and institutions. My very sincere gratitude goes to my supervisor, Professor G. Ranalli, for his support and advice throughout the whole program. His guidance and encouragement have been essential.

Dr. M. K. Paul of the Geological Survey of Canada is thanked for his contribution to the development the graphic processing program used in this thesis, which is a modification of a plotting code kindly provided by Dr. J. Melosh of the University of Arizona. Professor J.-C. De Bremaecker of Rice University and Dr. A. Yin of the University of California at Los Angeles offered valuable suggestions for the improvement of Chapter 3. I have also benefited from numerous discussions with Drs. A. Lambert, T. James, and K. Wang of the Geological Survey of Canada and Drs. Z. Yin, Z. Yue, and Mr. Z. Deng at Carleton University.

This thesis was financially supported by NSERC grants to Professor G. Ranalli and by Carleton University Scholarships to myself.

Most importantly I wish to thank my wife Jian Song and my daughter Shirley for their unwavering support, their love and sacrifices. My final thanks are extended to my parents for their persistent encouragement. 


\section{Original Contribution}

This thesis contains only the results of the research conducted by the candidate, under the guidance of his supervisor. Chapter 3 has been published in a slightly different form (see footnote on page 32). The candidate is the senior author of this paper, and the supervisor's (second author) contribution has consisted of advice and cooperation in the writing of the paper. 


\section{Table of Contents}

Title Page

Acceptance Sheet

Abstract

Acknowledgments

iii

Original Contribution

vi

Table of Contents

List of Figures

List of Tables

Chapter 1 Introduction

Chapter 2 Orogenic Wedge Models: A Review 4

2.1 Introduction 4

2.2 Orogenic belts, foreland fold-and-thrust belts, and accretionary prisms

2.3 Major characteristics of orogenic wedges 12

$\begin{array}{ll}2.4 \text { Previous work } & 12\end{array}$

2.4.1 Models for individual thrust sheets 13

$\begin{array}{lll}\text { 2.4.2 Continuum models for orogenic wedges } & 14\end{array}$

2.5 Rheology of orogenic wedges 28

Chapter 3 Stresses in An Overthrust Sheet and Propagation of Thrusting

Along Its Base

3.1 Introduction 32

3.2 State of stress within the thrust sheet 35

3.3 State of stress on the basal thrust 47

3.3.1 Nonlinear component of horizontal compression 48

3.3.2 Upper and lower slopes $\quad 50$

3.4 Discussion: initiation and development of thrusting 53

3.5 Very large wedges and the effect of fluid pressure 56

Chapter 4 Two-Dimensional Incompressible Power-law Flow 
4.1 Introduction 61

4.2 Model geometry and rheology 61

4.3 Basic equations 65

4.4 Derivation of solutions 66

$\begin{array}{lll}\text { 4.4.1 Stress solution } & 67\end{array}$

$\begin{array}{lll}\text { 4.4.2 Strain rate solution } & 70\end{array}$

$\begin{array}{lll}\text { 4.4.3 Velocity solution } & 71\end{array}$

4.5 Determination of functions $I(x)$ and $H(y) \quad 72$

4.6 The function $C(x, y)$ in the case of Newtonian rheology 77

Chapter 5 A Linear Longitudinal Strain Rate Solution For Power-Law Rheology 86

$\begin{array}{lll}5.1 & \text { Introduction } & 86\end{array}$

5.2 Model geometry and rheology 87

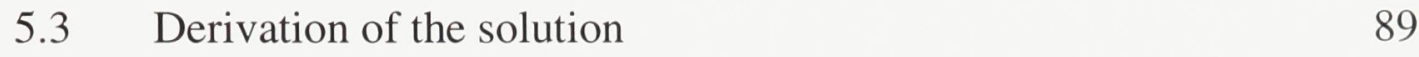

$\begin{array}{lll}\text { 5.3.1 Stress solution } & 89\end{array}$

$\begin{array}{lll}\text { 5.3.2 Strain rate solution } & 90\end{array}$

$\begin{array}{lll}\text { 5.3.3 Velocity solution } & 92\end{array}$

5.4 Implementation of analytical solution 99

5.4.1 General considerations 99

5.4.2 Wedge segmentation 100

5.4.3 Mutual dependence of the function $F(y)$ and the strain rate field 101

5.4.4 Solution implementation 103

5.4.5 Flowchart and numerical considerations 105

5.5 Comparison between two-dimensional and one-dimensional solutions 109

5.6 Comparison between the two-dimensional power-law solution and Platt's model 136

Chapter 6 Orogenic Wedges With Viscous, Power-law, and Plastic Rheologies 140

$\begin{array}{lll}6.1 & \text { Introduction } & 140\end{array}$

6.2 Models: geometry, composition and temperature $\quad 140$

6.3 Orogenic wedges with linearly viscous and power-law rheologies 
6.4.1 Low-temperature plastic upper layer

6.4.2 Effects of model parameters on the dynamics of orogenic wedges with plastic upper layer $\quad 162$

6.4.3 Orogenic wedges with various deformation patterns 176 6.4.3.1 Extensional wedge 180

6.4.3.2 Compressional wedge 184

6.4.3.3 Wedge with rear extension and frontal compression

Chapter 7 Conclusion

7.1 Main results

7.2 Discussion 


\section{List of Figures}

Figure 2.1 World map, showing the location of some major orogenic belts 5

Figure 2.2 Cross section across a model composite orogenic belt 5

Figure 2.3 Cross sections of four foreland fold-and-thrust belts and accretionary wedges 8

Figure 2.4a Three cross sections of the Alpine orogen 9

Figure 2.4b Geological cross section through the Himalayas 11

$\begin{array}{lll}\text { Figure 2.5 A simplified accretionary wedge model } & 15\end{array}$

$\begin{array}{lll}\text { Figure 2.6 Schematic diagram illustrating a bulldozer wedge } & 15\end{array}$

Figure 2.7 Notation and positive sense of stress components in the ice sheet model 24

Figure 2.8 Depth distributions of dimensionless stress, velocity, and displacement from Nye's one-dimensional theory for different stress exponent $n$

Figure $3.1 \quad$ Geometry of wedge-shaped thrust sheet under horizontal compression 36

Figure 3.2 Principal stress trajectories within the wedge-shaped thrust sheet subject to nonlinear horizontal compression on the rear vertical edge 45

Figure 3.3 Deviation of vertical normal stress from lithostatic values for selected model parameters

Figure $3.4 \quad$ (a) Normal and (b) shear stresses along the lower boundary of a rectangular block for four different sets of force boundary conditions

Figure $3.5 \quad$ (a) Normal and (b) shear stresses along the lower boundary of a wedge-shaped block for linear boundary forces and four different sets of parameters

Figure $3.6 \quad$ (a) Normal and (b) shear stresses along the lower boundary of a wedge-shaped block subject to nonlinear boundary forces for four different sets of parameters

Figure 3.7 Variation of frictional function along the basal thrust

Figure $3.8 \quad$ (a) Effective normal stress, (b) shear stress, and (c) frictional function along the basal boundary of a 100-km long wedge-shaped thrust sheet

Figure 3.9 Minimum pore fluid factor $\lambda$ as a function of rear thickness of the 
thrust sheet

Figure 4.1 Simplified two-dimensional orogenic wedge model

Figure 4.2 Boundary conditions along the basement AB 82

Figure 5.1 State of stress along the wedge rear boundary 91

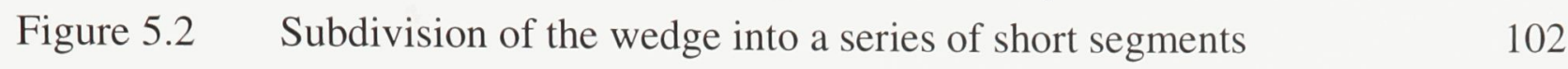

Figure 5.3 A short segment of wedge with assigned velocity boundary conditions on top and basal shear stress on bottom

Figure 5.4 A simplified flowchart for the implementation of the solution to equations (5.30) for each individual wedge segment

106

Figure 5.5 Parallel-sided rectangular block with power-law rheology and basement slope $\alpha$

Figure 5.6 Stress and deformation within the block for case A (Table 5.3)

Figure 5.7 Stress and deformation within the block for case B (Table 5.3)

Figure 5.8 Stress and deformation within the block for case C (Table 5.3)

Figure 5.9 Stress and deformation within the block for case D (Table 5.3)

Figure 5.10 Stress and deformation within the block for case E (Table 5.3)

Figure 5.11 Stress and deformation within the block for case F (Table 5.3)

Figure 5.12 Simplified wedge model

Figure 6.1 An orogenic wedge model parameterized by surface slope $(\alpha)$, basal slope $(\beta)$ and width $(\mathrm{L})$

Figure 6.2 Stress and deformation within the wedge for case A1 of Table 6.2

Figure 6.3 Stress and deformation within the wedge for case B1 of Table 6.2

Figure 6.6

A general power-law creep model $\left(\dot{\varepsilon}_{E}=\lambda \sigma_{E}^{\prime n}\right)$ with variable stress

\section{Figure 6.7} exponent $n$ and corresponding stress-strain rate graphs 


\section{List of Tables}

Table $3.1 \quad$ Values of model parameters for rectangular block with dimensions $\mathrm{L}=10 \mathrm{~km}, \mathrm{H}=1 \mathrm{~km}$ and density $\rho=2800 \mathrm{~kg} \mathrm{~m}^{-3}$ subject to nonlinear boundary forces

Table $3.2 \quad$ Values of model parameters for wedge-shaped block with dimensions of $\mathrm{L}=10 \mathrm{~km}$ and $\mathrm{H}=1 \mathrm{~km}$ subject to linear boundary forces

Table 3.3 Values of model parameters for wedge-shaped block with dimensions $\mathrm{L}=100 \mathrm{~km}, \mathrm{H}=10 \mathrm{~km}$ subject to nonlinear boundary forces and $\lambda=0.75$ on the basal thrust

Table 4.1 Steady state power-law rheology and deformation patterns

Table 5.1 Physical significance of function and constants appearing in equations (5.30)

Table 5.2 Comparison between Nye's one-dimensional solution and the present two-dimensional solution

Table 5.3 Values of model parameters for rectangular block with length $\mathrm{L}=250 \mathrm{~km}$, depth $\mathrm{H}=40 \mathrm{~km}$ and constant density $\rho=2800$ $\mathrm{kg} \mathrm{m}^{-3}$ over a basement with slope angle $\alpha=3^{\circ}$

Table 6.1 Steady-state power-law creep parameters for crustal materials (Ranalli \& Murphy, 1987; data modified from Kirby, 1983)

Table 6.2 Model parameters for orogenic wedges with width $\mathrm{L}=200 \mathrm{~km}$ and top and basal slopes $\alpha=3^{\circ}$ and $\beta=6^{\circ}$ (constant density $\rho=2700 \mathrm{~kg} \mathrm{~m}^{-3}$ )

Table 6.3 Parameters for viscoplastic wedges with $\mathrm{L}=200 \mathrm{~km}$ and top shortening rate increasing towards the front

Table 6.4 Parameters for an anorthosite wedge with $\mathrm{L}=200 \mathrm{~km}, \alpha=3^{\circ}$ and $\beta=6^{\circ}$, subject to a geothermal gradient of $15 \mathrm{~K} \mathrm{~km}^{-1}$ 


\section{Chapter 1}

\section{Introduction}

Orogenic belts provide the geological record of mainly compressive plate tectonic activity, including subduction of one plate beneath another, and collision between crustal masses, such as two continents, a continent and an island arc, or a continent and an oceanic plateau. Structurally, orogenic belts display a variety of styles, but they always include roughly wedge-shaped regions (in cross section), with or without bilateral symmetry, verging towards the foreland. These wedge-shaped regions are termed orogenic wedges.

Within the framework of plate tectonic theory, orogenic belts are interpreted as forming at convergent margins as a result of subduction and collision of two plates. Thus study of an orogenic belt provides vital information for the understanding of the interaction between two tectonic plates. There are basically two different points of views in studying the dynamics of orogenic wedges: on a smaller scale, foreland thin-skinned thrusts are envisioned as rigid blocks moving on discrete faults; on a larger scale, both thin- and thick- skinned wedges are considered as a single, mechanically continuous, deforming body. Considerable progress has been made in the past three decades, regarding the dynamics of orogenic wedges and their tectonic evolution. However, there are still many problems which are not well understood. This thesis deals with stress and deformation in orogenic wedges from two different perspectives: (1) brittle, dislocationtype propagation of thrusting along the base of a single thrust sheet; (2) dynamics of orogenic wedges as a whole with generalized creep rheology. Both treatments are analytical and quantitative from a continuum mechanics approach. 
Chapter 2 provides a review on orogenic wedges, including tectonic background, mechanical and dynamic characteristics, and outlines some of the major problems which are dealt with in this thesis.

An elastic solution is derived in Chapter 3 to study the stresses in an individual wedge-shaped thrust sheet. This solution is used to justify the progressive, consecutive "dislocation-like" propagation of the basal thrust, and consequently provides a quantitative explanation for the mechanical paradox of overthrusting.

Chapters 4, 5, and 6 are devoted to the study of orogenic wedges with a generalized power-law creep rheology. A two-dimensional solution for a wedge-shaped block is derived in Chapter 4. Stress, strain rate and velocity fields are expressed explicitly in terms of the bulk rheology of wedge material, the force boundary condition at the wedge's rear boundary, and a two-dimensional function representing the deviation of vertical normal stress from the lithostatic pressure. A special case for a wedge with linear Newtonian rheology is discussed in detail to show how to determine deviations from lithostatic conditions from other boundary conditions.

A two-dimensional analytical solution is given in Chapter 5 for an orogenic wedge with nonlinear steady-state rheology and a longitudinal strain rate varying linearly with distance from the rear of the wedge. Stress, strain rate, and velocity components are functions of bulk rheology, temperature distribution, and arbitrary constants determinable from dynamic and/or kinematic boundary conditions. Several cases dealing with parallelsided blocks are discussed in detail, and the results are compared with one-dimensional solutions.

Chapter 6 presents computational results for several wedge-shaped block cases based on the two-dimensional solution derived in Chapter 5. These cases are classified into two groups based on the rheological characteristics of the wedge: the first group deals with 
wedges with viscous and power-law rheologies; the second group considers wedges with top plastic and bottom power-law rheologies.

Final discussion and conclusions about this two-dimensional power-law orogenic wedge model form the main part of Chapter 7. Also included in this chapter is a brief discussion on the possible further extensions of the analytical study of orogenic wedges reported in this thesis. 


\section{Chapter 2}

\section{Orogenic Wedge Models: A Review}

\subsection{Introduction}

This chapter begins with a brief description of orogenic belts and wedges within the framework of plate tectonics. Then, the major developments in the evolution of our understanding about the dynamics of orogens are reviewed. Next, the rheological characteristics of orogenic wedges are discussed. Finally, the objectives of this thesis are outlined at the end of the chapter.

\subsection{Orogenic belts, foreland fold-and-thrust belts , and accretionary prisms}

Orogenic belts, coinciding with some of the Earth's great mountain chains, represent the geological record of mainly compressive plate tectonic activity. This activity includes subduction of an oceanic plate beneath a continental or oceanic plate, as well as collision between two continental plates (including an island arc, or an oceanic plateau). Orogenic belts are zones of intense deformation ( $c f$. Suppe, 1985 for a general discussion). Active orogenic belts are localized along plate boundaries and commonly along continental margins, apparently reflecting a fundamental instability of continental margins relative to oceanic and continental interiors. Figure 2.1 shows the location of some major Phanerozoic orogenic belts, chiefly the American Cordillera, and the Appalachian, Caledonide, and Alpine-Himalayan systems. 


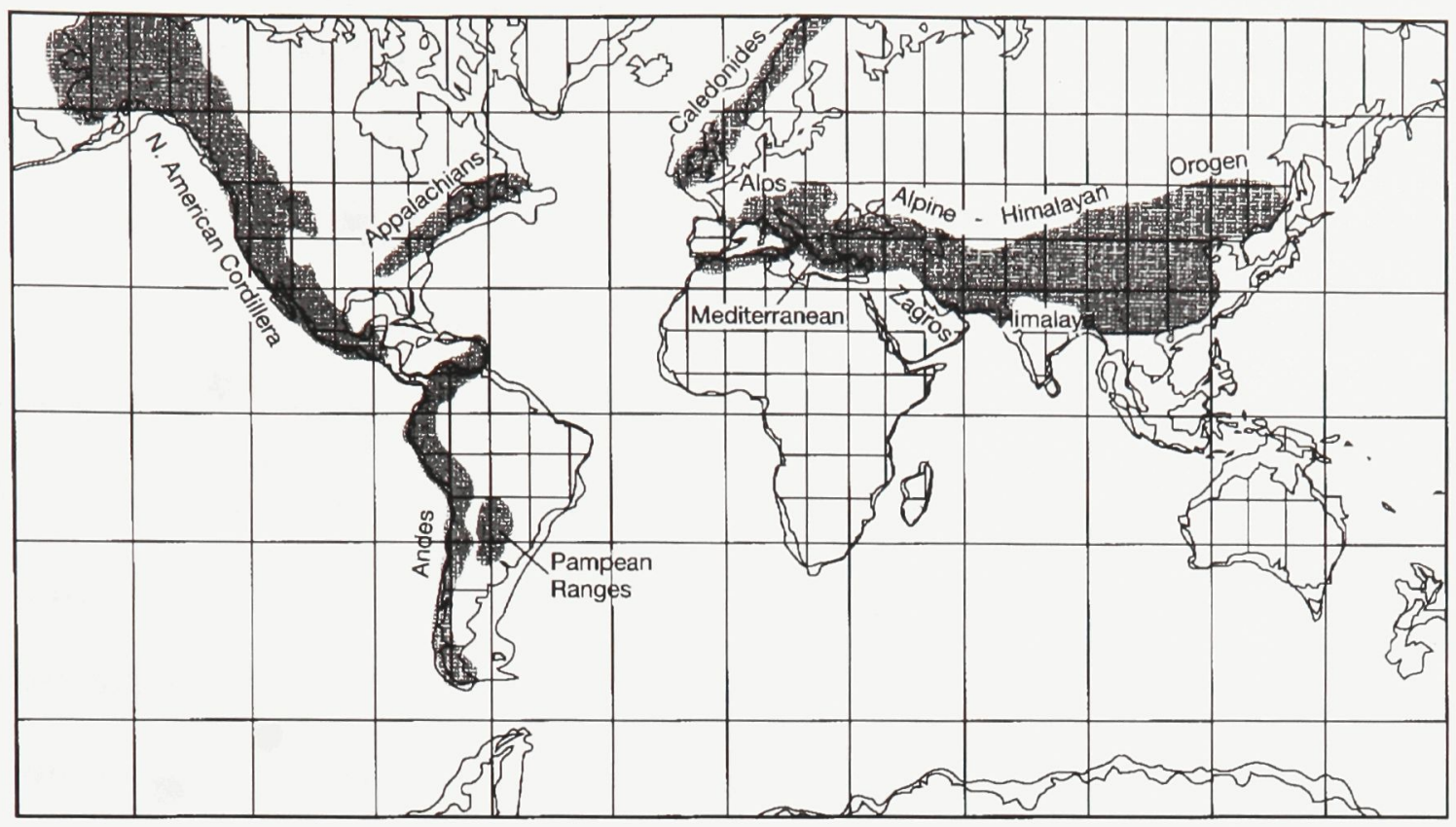

Figure 2.1 World map, showing the location of some major orogenic belts (from Twiss and Moores, 1992).
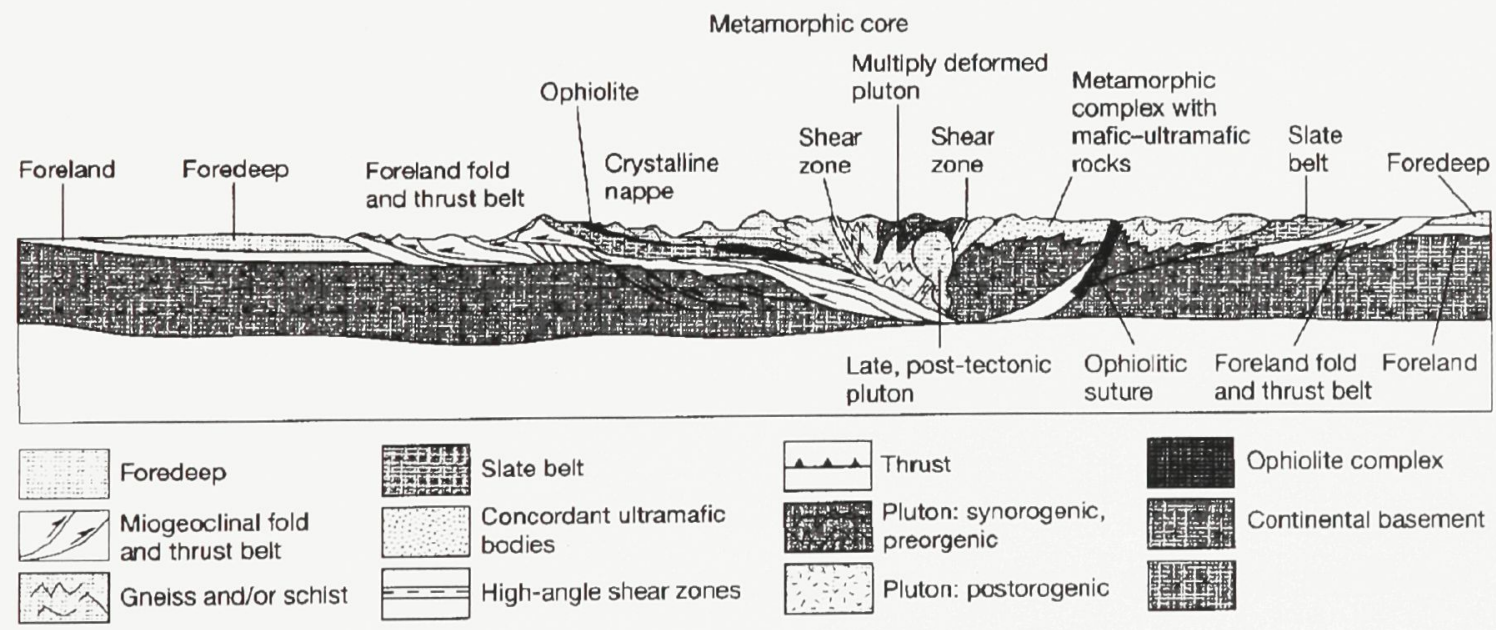

Figure 2.2 Cross section across a model composite orogenic belt (from Twiss and Moores, 1992). 
Although significant variations can be found among cross sections of different orogenic belts ( $c f$. Twiss and Moores, 1992), they have a number of features in common: a rough bilateral structural (but not geometrical) symmetry; a foreland or undeformed plate on either side; outer foredeeps; fold and thrust belts; sutures marked by ophiolitic rocks; and an internal crystalline core zone of metamorphosed and deformed sedimentary and volcanic rocks, mafic-ultramafic complexes, and granite plutons (Figure 2.2).

Because of the complex structural character of orogenic belts, it is natural to consider only simplified models, for instance the wedge-shaped area (in cross section) between the foreland and the centre of the belt. This part of the orogenic belt is often referred to as the orogenic wedge (Pavlis and Bruhn, 1983; Platt, 1986; Dechesne and Mountjoy, 1992; Royden, 1993; Yin, 1993).

As parts of orogenic belts, foreland fold-and-thrust belts are belts in which the tectonic style is dominated by overthrusting, i.e., tectonic emplacement of sheets of allochthonous rocks over autochthonous material (Boyer and Elliott, 1982; Price and Cosgrove, 1990). They are 'foreland' because they usually occupy an exterior position (relative to the direction of tectonic transport) with respect to the structural and metamorphic culmination of the orogen. Foreland fold-and-thrust belts usually lie on the continental side of the orogen. Accretionary prisms, forming above oceanic subduction zones, are deformed wedge-shaped sedimentary masses between the front trench line and the rear outer arc high ( $c f$. Fowler, 1990 for detailed description on subduction zones and accretionary prism).

In this thesis, the term orogenic wedge is used in a general sense for both thin- and thick-skinned wedges, while thin-skinned orogenic wedge is reserved for foreland foldand-thrust belts not affecting the basement, and accretionary prisms.

Typical examples of foreland fold-and-thrust belts and accretionary prisms include: 
(a) the Barbados accretionary complex, located to the east of the Lesser Antilles island arc, where $65 \mathrm{Ma}$ oceanic lithosphere of the North American plate is subducting beneath the Caribbean plate (Westbrook, 1982; Behrmann et al., 1988) (Figure 2.3a);

(b) the Taiwan fold-and-thrust belt produced by the subduction of the Eurasian plate beneath the Philippine Sea Plate (Suppe, 1980)(Figure 2.3b);

(c) the southern Canadian Cordillera fold-and-thrust belt formed during the late Jurassic and Cretaceous, and involving several interrelated processes including accretion of outboard terranes to the western margin of North America and protracted compression and crustal thickening (Price, 1981; Brown et al., 1992)(Figure 2.3c).

(d) the southern Appalachians deformed during the late Carboniferous to Permian Alleghenian orogeny (Figure 2.3d).

According to Pavlis and Bruhn (1983), thin-skinned and thick-skinned orogenic wedges may represent different stages of the evolution of orogenic belts in subducting regions. Thin-skinned wedges, such as the Barbados accretionary prism, could be the early product of subduction, when the whole complex is in its initial stages of development and consists mainly of the accretionary prism. With the growth of the subduction complex, the horizontal and vertical dimensions of the wedge increase. Thickskinned wedges are more ductile and metamorphosed than thin-skinned wedges. Some typical thick-skinned orogenic wedges include:

(a) the Alpine orogen, a consequence of the convergence between Europe and Adria, a small plate that includes the continental lithosphere underlying Italy and the Adriatic Sea (Polino et al., 1990)(Figure 2.4a);

(b) the Himalayan orogen developed during the collision of the Indian plate with Eurasia (Burg and Chen, 1984; Burchfiel and Royden, 1985; Burchfiel et al., 1992; Harrison et al., 1992)(Figure2.4b). 


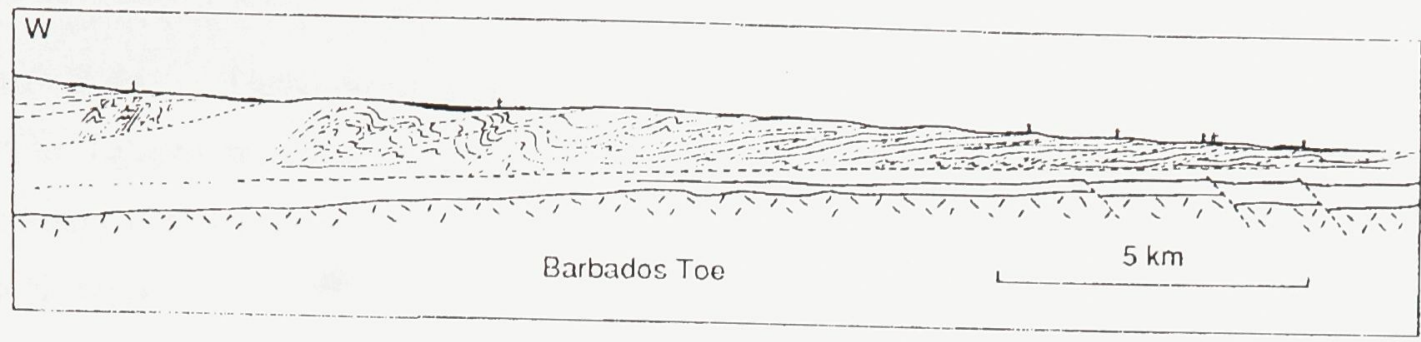

(a)

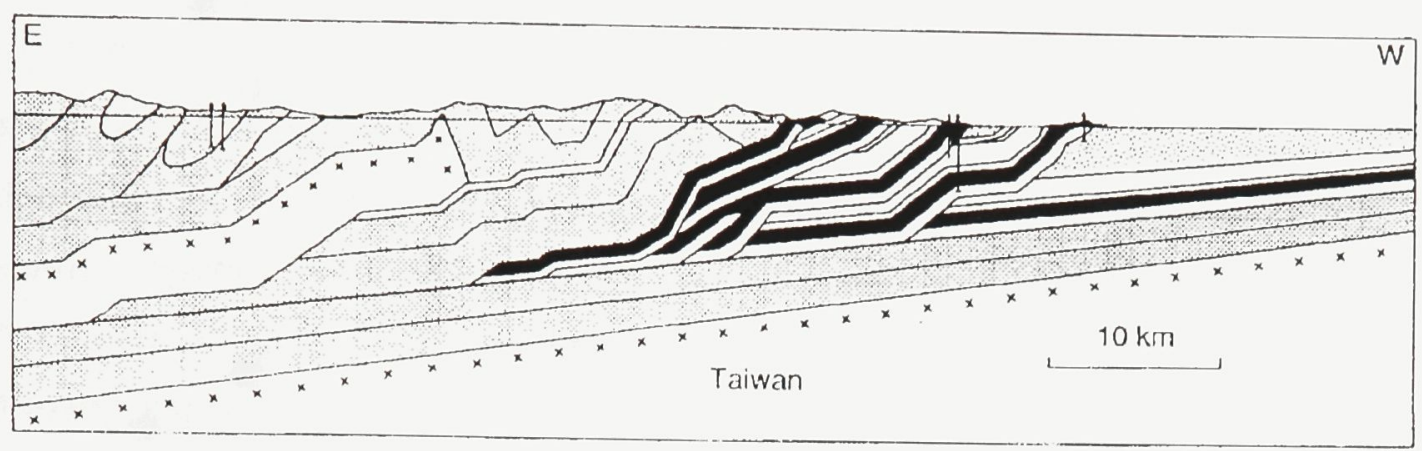

(b)

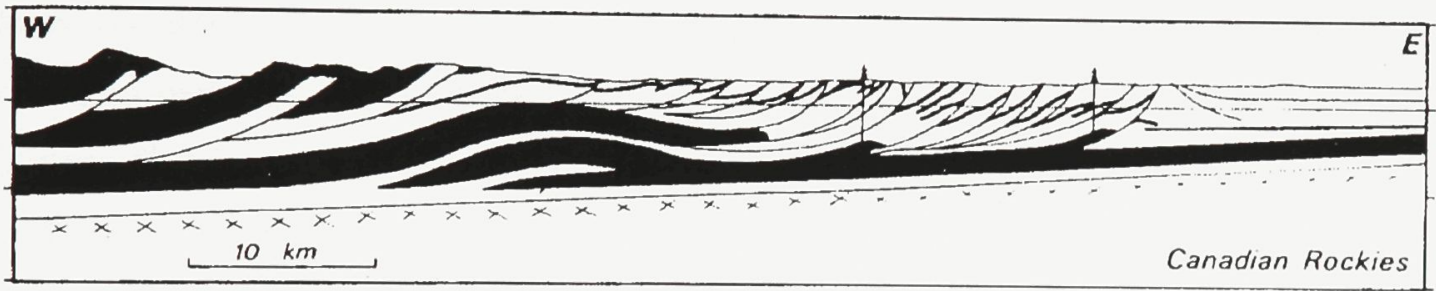

(c)

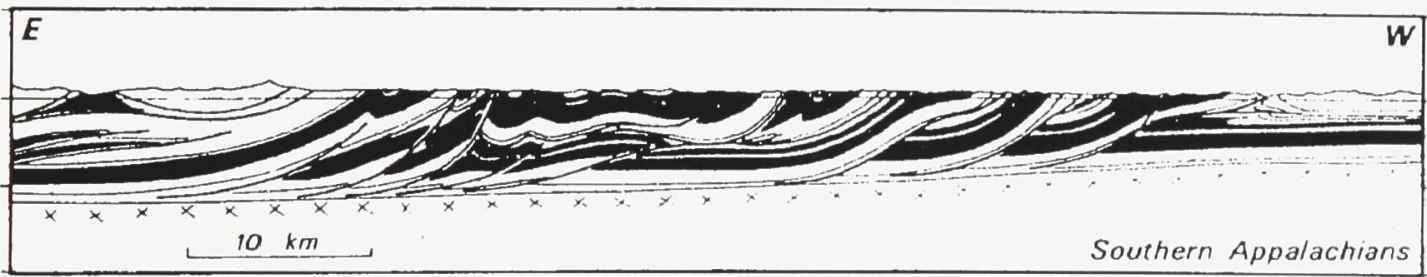

(d)

Figure 2.3 Cross sections of four foreland fold-and-thrust belts and accretionary wedges: (a) Barbados accretionary wedge; (b) Taiwan foreland fold-and-thrust belt; (c) Canadian Rockies; (d) Southern Appalachians. No vertical exaggeration (from Dahlen, 1990). 
Figure 2.4a Three cross sections of the Alpine orogen: (1) the eastermost Austrian Alps, (2) the Tauern window, and (3) the central Alps. AD: Adula nappe; AU: Austroalpine basement and cover nappe system of the eastern Alps; BE/RE/TW: Bernstein, Rechnitz and Tauern windows; CA: Canavese zone; GO/TV/AAR/MB/BE/AG: Gotthard, Tavetsch, Aar, Mont Blanc, Belledonne and Argentera Helvetic-Dauphinois basement units; HE: Utrahelvetic, Helvetic and Dauphinois cover units; HF: Helmintoid flysch of the French-Italian Alps; LPN: Simplon-Tessin lower Penninic nappes; NCA: northern calcareous Alps, Austroalpine; P: Prealpine décollement nappes; PA/GL/MA/PI/AN: Platta-Arosa, Glockner, Malenco-Avers, Piedmont and Antrona ophiolitic units; UP/TN/SU/MR/GP/DM: Upper Penninic, Tauern, Suretta, Monte Rosa, Gran Paradiso, and Dora Maira basement nappes; SA: southern Alps; TA/SB/PN: middle Penninic Tambò, Grand St. Bernard/Briançonnais and Pinerolese nappes; VA: Valais (North-Penninic) ophiolitic and flysch units; PF: Penninic thrust front; PL: Periadriatic (Insubric) fault system. 1: European foreland; 2a: Eastern Austroapline Eoalpine very low grade metamorphism; 2b: Eastern Austroapline Eoalpine greenschist and amphibolite facies metamorphism; 3: ophiolitic units, related metamorphic flysch and mélange; 4: flysch décollement units, mostly Cretaceous; 5a: Tertiary European molasse; 5b: Tertiary Po plain molasse; 6: Periadriatic plutons, mostly Oligocene (from Polino et al., 1990). 

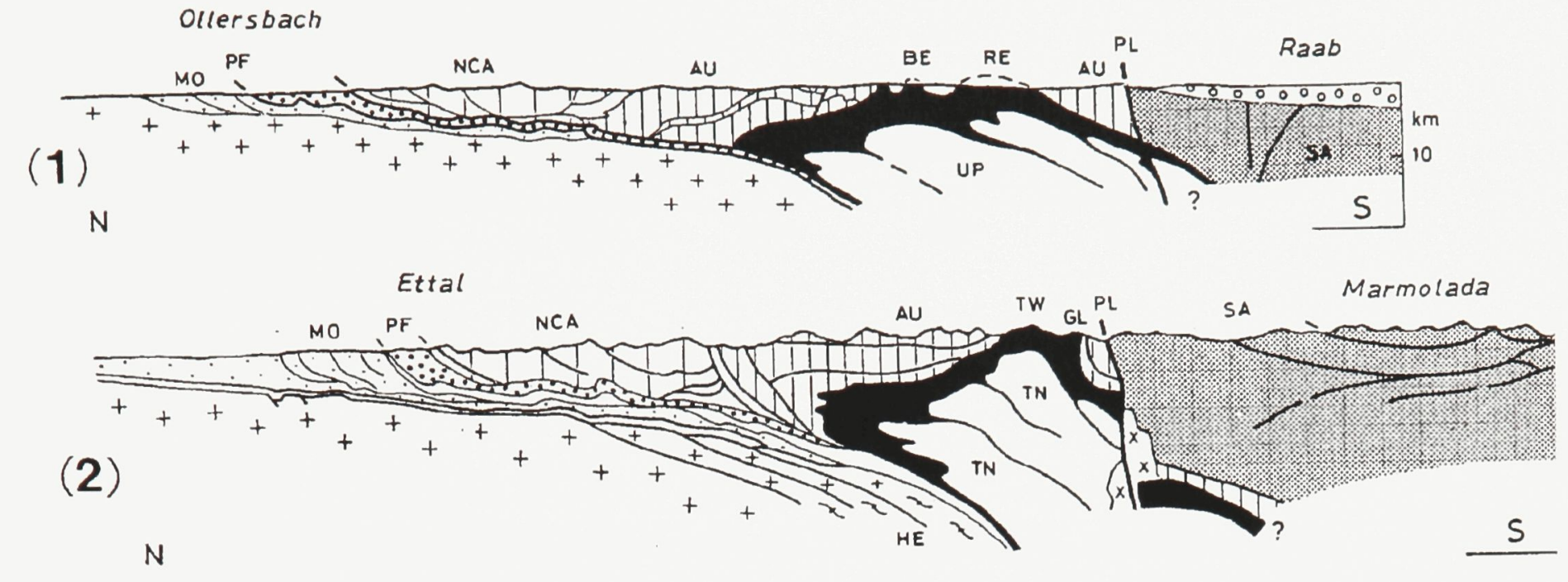

(3)
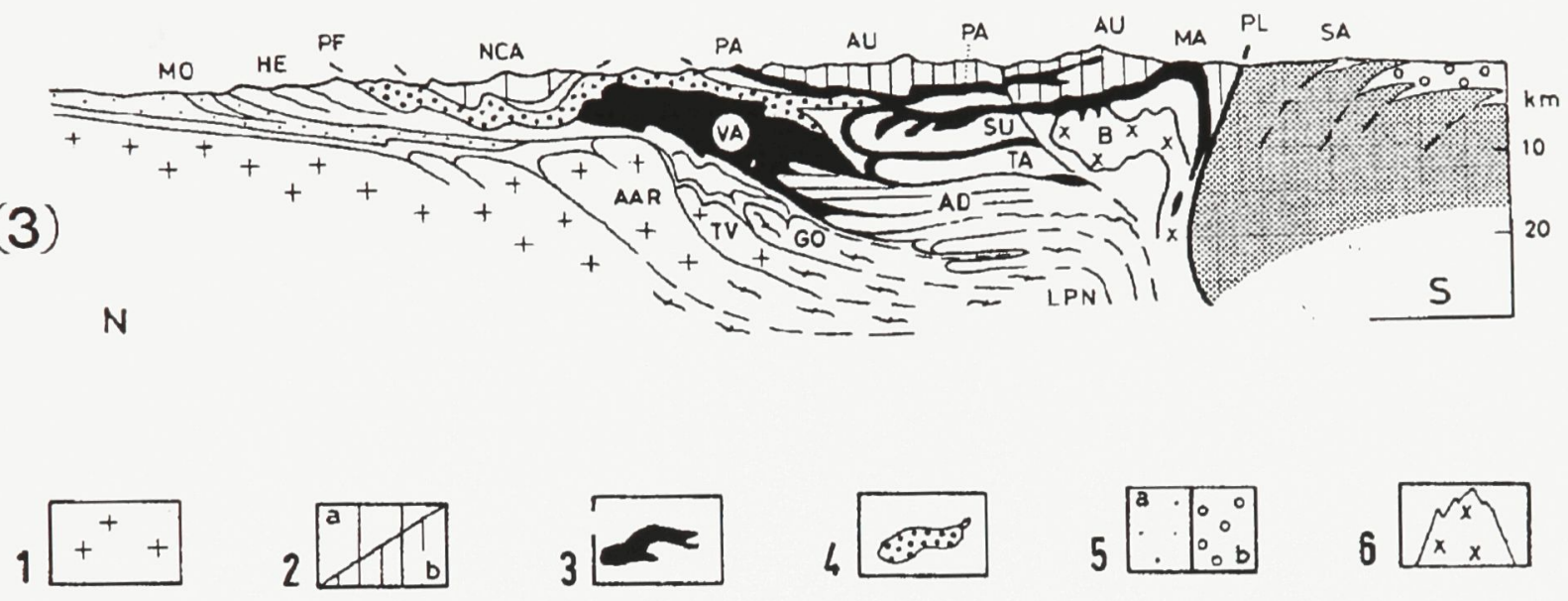

${ }_{0}\left[\begin{array}{ll}x_{2} x \\ x\end{array}\right.$ 


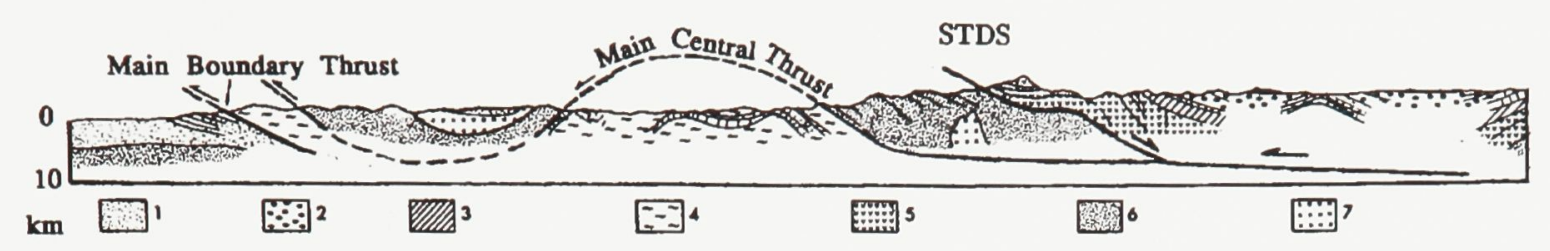

Figure 2.4b Geological cross section through the Himalayas, simplified from Gansser (1964) and Lyon-Caen and Molnar (1983). STDS: south Tibetan detachment system of Burchfiel et al. (1992). 1: Tertiary and Quaternary sedimentary rocks; 2: Mesozoic Indus flysch; 3: Paleozoic sedimentary rocks of Greater Himalaya; 4: Paleozoic sedimentary rocks of Lesser Himalaya; 5: upper Precambrian and lower Paleozoic sedimentary rocks; 6: Precambrian basement; 7: Himalaya leucogranites. 


\subsection{Major characteristics of orogenic wedges}

The main characteristics of thin-skinned orogenic wedges are (McClay, 1992; Ranalli, 1995):

(a) Wedge-shaped geometry, thicker at the rear, which is the direction of provenance of the thrust sheets;

(b) Deformation beginning earlier at the rear than at the front, with a "piggy-back" mode of transport, in which the lowest thrust plane is the most recent and consequently the highest thrust sheets have travelled the farthest;

(c) Lateral shortening and vertical thickening as the main deformation characteristics;

(d) A weak basal layer (e.g., evaporites, high pore fluid pressure) sometimes (but not always) occurring between the thrust belt and the basement.

Thick-skinned orogenic belts may additionally display the following features (Pavlis and Bruhn, 1983; Platt, 1986,1987, 1993):

(a) Coeval development of compressional structures at the thin-skinned foreland "front" and extensional structures at the thick-skinned "rear" towards the hinterland;

(b) Exhumation of high pressure-low temperature metamorphic rocks near the rear of the wedge, while convergence is continuing;

(c) Increasing ductility of the rock material towards the rear low corner of the orogenic complex, due to higher temperature.

\subsection{Previous work}

Several models (numerical and analytical) have been proposed to analyze the deformation processes in orogenic wedges. Conceptually, these models can be divided into two groups: the first group focuses on the stress and deformation in individual thrust 
sheets; the second group assumes that the entire wedge (thin-skinned and/or thickskinned) behaves as a single, mechanically continuous, unit, and studies its overall evolution.

\subsubsection{Models for individual thrust sheets}

One of the most debated questions in modelling the movement of thrust sheets deals with the famous "mechanical paradox of overthrusting" (Reade, 1908; Smoluchowski, 1909). This paradox can be illustrated by studying a rectangular block resting on a horizontal base ( $c f$. Suppe, 1985; Ranalli, 1995). Assuming that the block is of uniform density $\rho$, pore fluid factor (ratio of pore fluid pressure to lithostatic pressure) $\lambda$, height $h$ and length $l$, and that the underlying base has cohesion $S_{0}$, frictional coefficient $\mu_{0}$ and pore fluid factor $\lambda_{0}$, consideration of force balance allows estimation of the maximum length of the thrust sheet that can move as a coherent unit. This is given by

$$
\frac{l_{\max }}{h}=\frac{2 C+[R(1-\lambda)+\lambda] \rho g h}{2 S_{0}+2 \mu_{0} \rho g h\left(1-\lambda_{0}\right)}
$$

where $R$ is the stress ratio between the maximum and minimum compressional stresses, and $C$ is the uniaxial compressive strength of the block, related to the block's cohesion $S$ and frictional coefficient $\mu$ as $C=2 S\left[\left(1+\mu^{2}\right)^{1 / 2}+\mu\right]$. If the parameters of the block are the same as those at its base, the right-hand side term in equation (2.1) is about 3 (cf. Suppe, 1985). This result strictly limits the maximum length of overthrust sheets (physically, this is a consequence of the fact that the pushing force that can be applied at the rear is limited by the strength of the block). To explain the observations of overthrusts with lengths of the order of tens or hundreds of kilometers and thicknesses of only a few kilometers, therefore, very high pore fluid pressures and/or weak layers are required at 
their bases. When evidence for such pressure or weak layers is lacking, the occurrence of large overthrusts is difficult to explain. This is the so-called 'mechanical paradox' for overthrusts.

In Chapter 3, following a suggestion by Price (1988), we offer a solution to the paradox based on the idea that the thrust propagates with finite speed and does not slide at the same time throughout its length. An Airy stress function solution is derived for incomplete boundary conditions, and computational results from this solution show that the ratio of basal shear stress to basal normal stress decreases from the rear to the front of thrust plane for wedge-shaped thrust blocks, which means that movement of a large coherent overthrust sheet may be realized by a progressive, dislocation-type thrust faulting along its base.

\subsubsection{Continuum models for orogenic wedges}

A fundamental concept in these models is that the wedge behaves as a single, mechanically continuous, dynamic unit (Platt, 1986). This was argued explicitly by Price (1973a) who suggested that the wedge as a whole is macroscopically ductile in that it does not lose its overall mechanical integrity during deformation.

Most previous studies have focused on the dynamics of thin-skinned orogenic wedges which are generally less than $100 \mathrm{~km}$ in width and 10 to $15 \mathrm{~km}$ in depth at the rear (as an order of magnitude). All the models proposed in these studies have been trying to account for the deformation of the wedge in terms of the following parameters:

(a) the overall geometry of the wedge (i.e., top and basal slopes; see Figure 2.5);

(b) its internal mechanical strength; and

(c) the mechanical strength of the material between the wedge and the underlying basement. 


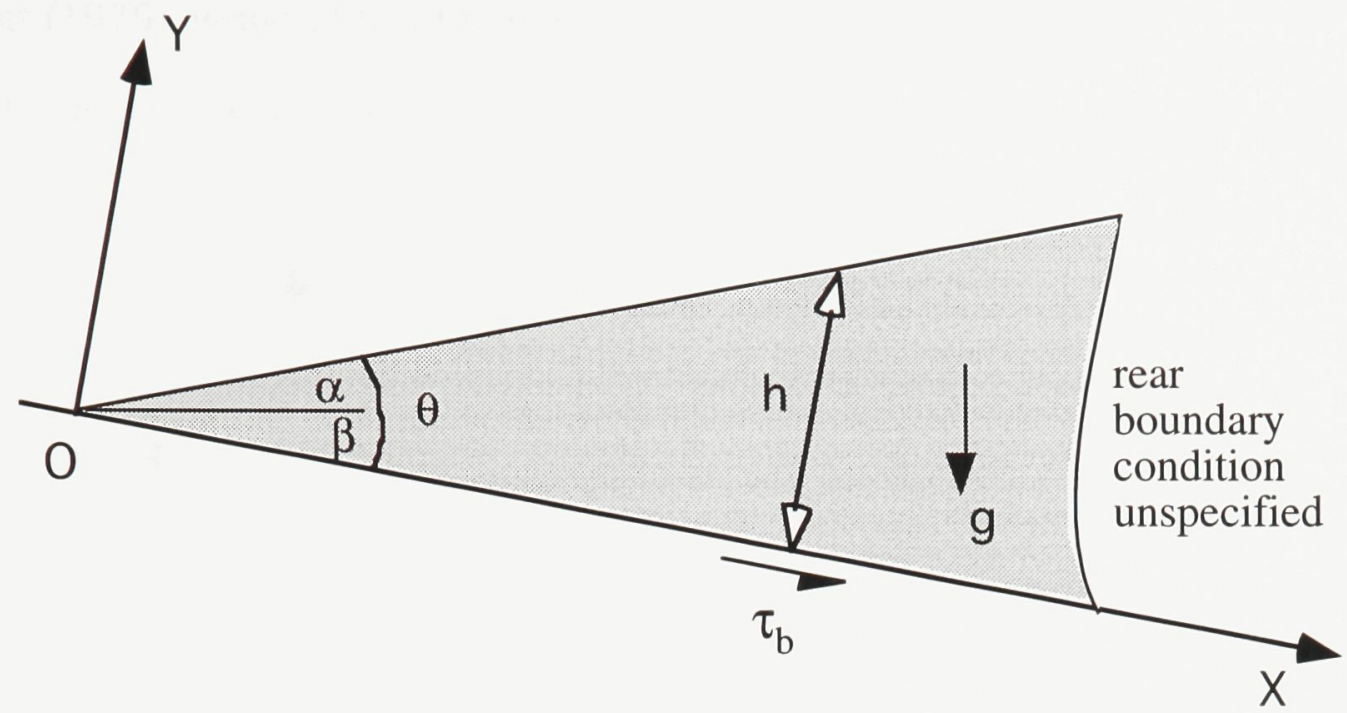

Figure 2.5 A simplified accretionary wedge model (Platt, 1986): $\alpha$ and $\beta$ are the top and basal slopes, respectively; $\theta(=\alpha+\beta)$ is the taper of the wedge; $h$ is the thickness of the wedge and $\tau_{b}$ is the shear stress at the base of the wedge.

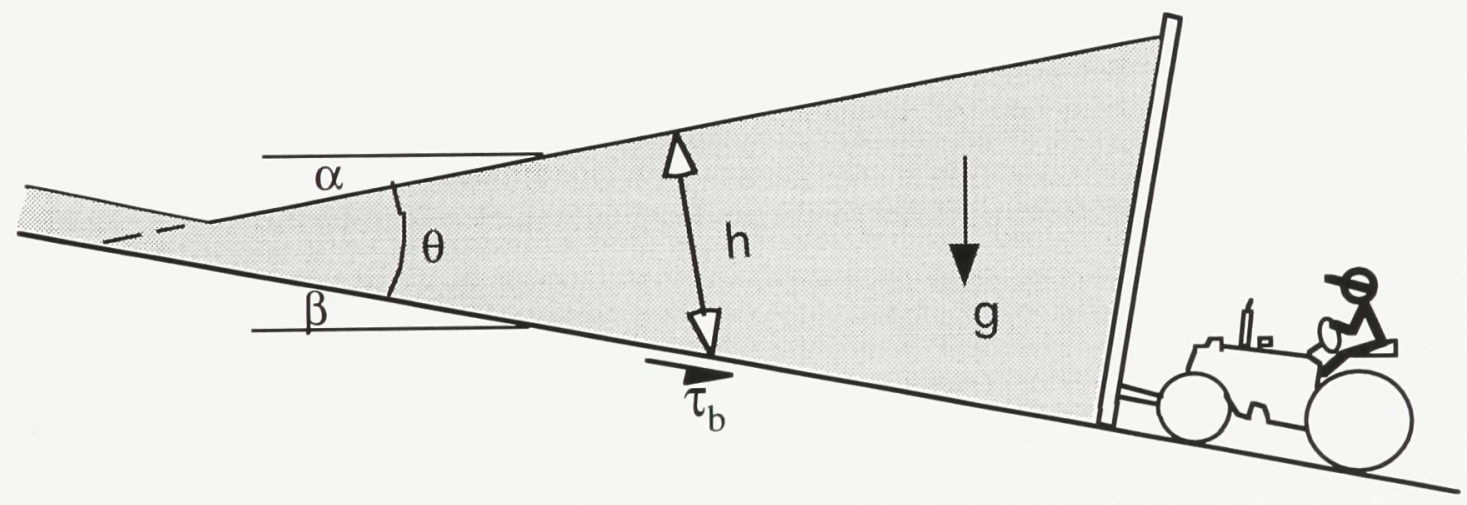

Figure 2.6 Schematic diagram illustrating a bulldozer wedge (from Dahlen, 1990). $\alpha$ and $\beta$ are the top and basal slope angles, respectively; $\theta(=\alpha+\beta)$ is the taper of the wedge; $h$ is the local thickness of the wedge at the location where $\tau_{b}$ is the shear stress at the base of the wedge. (Note that the definition of $h$ varies slightly with the choice of coordinate system.) 
Elliott (1976) assumed that the material in the wedge is relatively weak and unable to sustain deviatoric stresses of more than $20 \mathrm{MPa}$ on a geological time scale. Based upon this assumption, the deformation of wedge can be considered analogous to that of a glacier, and at equilibrium the basal resistance to the sliding flow of the orogenic wedge is balanced by the gravitational shear stress in the longitudinal direction

$$
\tau_{b}=\rho g h \alpha
$$

where $\tau_{b}$ is the shear stress at the base, $\rho$ the density of the wedge material, $g$ the gravitational acceleration, $\alpha$ the surface slope, and $h$ the local thickness of the wedge (see Figure 2.5). This is in perfect analogy with the flow of a glacier or ice-sheet (Paterson, 1981). According to equation (2.2), the wedge material spreads under the action of gravity. This gravity spreading model, however, is not by itself applicable to the analysis of foreland fold-and-thrust belts since it does not account for the pervasive shortening observed in thrust belts.

Contrary to the simple gravity spreading model, Chapple (1978) assumed that the wedge material is strong enough to stand a longitudinal stress of up to $200 \mathrm{MPa}$, and that there exists a relatively weak layer along the base of the wedge. By treating the wedge and the underlying weak basal layer as continua with perfectly plastic rheology, and assuming both wedge and weak layer to be at the yielding point throughout the whole block, the following expression can be derived relating the basal shear stress $\left(\tau_{b}\right)$ to the wedge's taper (i.e., surface slope $\alpha$ and basement slope $\beta$ ) and the plastic strength of the material $(K)$

$$
\tau_{b}=\rho g h \alpha+2 K \beta
$$


The basal shear stress, $\tau_{b}$, equals the plastic strength of the weak basal layer and can be expressed in terms of the strength of the wedge material as

$$
\tau_{b}=\chi K
$$

Thus, $\chi$ is equal to the ratio of the yield strength of the weak basal layer to that of the wedge. Equations (2.3) and (2.4) show that the resistance to sliding $\left(\tau_{b}\right)$ is balanced by two driving forces: gravity-induced lateral spreading and tectonic compression (the solution is strictly valid only for small surface and basement slopes). The most important conclusion from this model is that the wedge-shaped geometry is a consequence of internal dynamics. If the force applied at the rear of the wedge is counteracted by the resistance to sliding on its base, a forward topographic slope (and therefore a greater cross-section at the rear) is needed to prevent the longitudinal stress from exceeding the strength of the material. If the wedge is insufficiently tapered (that is, the sum of topographic and basal slopes is too small), it shortens internally and thickens at the rear until it is able to transmit the force from behind towards the front. This implies that the wedge material is at the yield point throughout. Equations (2.3) and (2.4) also show that the critical taper $\theta(=\alpha+\beta)$ decreases with decreasing strength of the basal layer, i.e., thin, long wedges require a weak base, while a strong base results in a larger critical taper. As a first approximation this model satisfies the characteristics of thin-skinned foreland belts.

The plastic model described above applies to thin-skinned wedges where the temperature is not sufficiently high to induce steady-state viscous flow. Whereas plastic flow does not begin until the stress level reaches some critical value, viscous flow is produced by any stress, no matter how small. 
Besides the perfect plasticity used in Chapple's model, another rheology applicable to low-temperature supracrustal thin-skinned wedges is the frictional Coulomb-Navier criterion (Jaeger and Cook, 1969; Paterson, 1981; Ranalli, 1995). This rheology, modified for the effects of pore fluid pressure, is assumed in the popular critical taper wedge model (Davies et al., 1983; Dahlen, 1984; Dahlen et al., 1984; Zhao et al., 1986; Dahlen, 1990). This model is basically analogous to a wedge of snow or sand in front of a moving bulldozer (Figure 2.6). Analogously to the plastic model, the state of stress within a critically tapered wedge is assumed everywhere on the verge of Coulomb-Navier failure, and an expression for the critical taper is derived in terms of material parameters. The essential premise of the critical taper model implies that (a) a critical wedge grows self-similarly; and (b) the wedge shortens or extends to regain the critical state if the taper becomes subcritical or supercritical. There are several expressions for the critical frictional taper, depending upon whether the effects of cohesion and/or pore fluid pressure are considered or not. In the simplest case, where the cohesion of wedge material is ignored and the internal friction coefficient is assumed to be constant within the wedge, the critical taper can be approximated as

$$
\alpha+\beta=\frac{1-\sin \phi}{1+\sin \phi}\left(\mu_{b}+\beta\right)
$$

where $\alpha$ and $\beta$ are the top and basal slope angles, respectively, $\phi$ is the angle of internal friction, and $\mu_{b}$ is the coefficient of basal friction. For typical values $\phi \approx 35^{\circ}$ $(\tan \phi \approx 0.7)$ and $\beta \approx 3^{\circ}-5^{\circ}$, the critical taper is $\alpha+\beta \approx 11^{\circ}-12^{\circ}$ if the basal frictional coefficient is equal to that of the material within the wedge. The critical taper decreases to $6^{\circ}$ if the angle of basal friction is about half of that of the material within the wedge.

The frictional critical taper model has gained a great deal of popularity because of its simple and concise expression relating the overall geometry of a supracrustal wedge to 
material parameters which are in principle measurable (e.g., cohesion, coefficient of friction and pore fluid pressure). This model has been applied to several well-studied thrust-and-fold belts and the predicted and measured observables are in reasonable agreement (Davies et al., 1983; Dahlen, 1984; Dahlen et al., 1984; Zhao et al., 1986).

The overall dynamics of the plastic model and of the frictional model are similar. Both models describe a dynamically stable state: once the critical taper of the wedge is reached, the wedge is everywhere at failure. As a matter of fact, the similarity between these two models is also evident from the expression linking the basal shear stress to the gravitational stress associated with topographic slope and the material properties of the wedge

$$
\begin{aligned}
& \tau_{b}=\rho g h \alpha+2 K_{1} \beta \\
& \tau_{b}=\rho g h \alpha+2 K_{2}(\alpha+\beta)
\end{aligned}
$$

The first of equations (2.6) refers to the plastic model, and the second to the frictional model. The parameter $K_{1}$ in the first equation represents the yield strength of the wedge, while the parameter $K_{2}$ in the second equation is a function of both the internal strength of the wedge and the ratio of pore-fluid to lithostatic pressure.

The frictional critical taper theory was first proposed by Davis et al. (1983), and since then its theoretical formulation has being modified to take into account the spatial variation in porosity, pore-fluid pressure, and other parameters (Zhao et al., 1986; Fletcher, 1989; Dahlen, 1990). One variation of this model simulates extensional accretionary wedges (Xiao et al., 1991). However, critical taper theory and its variations are only applicable to thin-skinned wedges (because of the assumed rheology) and predict a uniform stress state throughout the wedge. (Even for thin-skinned wedges, this model seems to be more applicable to accretionary prisms than to foreland fold-and-thrust belts 
(Woodward, 1987)). They are therefore not applicable to those wedges with synconvergence extension at the rear, as often observed in thick wedges.

Yin (1993) tried to overcome the second limitation of the critical taper model and derived an elastic Airy stress function solution. His model assumes frictional sliding along the base and a linear variation with depth of shear and normal tractions along the rear of the wedge. Using this model, the roles of basal friction, pore fluid pressure, and wedge configuration in controlling the mechanics of thrust wedges are evaluated. The model predicts that (1) relatively low basal frictional resistance leads to dominantly horizontal compression in the wedge, whereas relatively high basal frictional resistance leads to dominantly horizontal extension; (2) a long thrust wedge (>100 km) may show thrust faulting in its lower part and normal faulting in its upper part, even when a moderate to high compressive horizontal stress (100 $\mathrm{MPa})$ is applied at the front of the wedge, whereas a short thrust wedge $(<10 \mathrm{~km})$ can be entirely compressional under the same boundary conditions; (3) the topographic slope is less important than basal friction in affecting the deformational style.

The result on the relative importance of topographic slope and basal friction in producing extensional structures within compressional wedges is very interesting. However, this solution cannot be directly applied to thick orogenic wedges. First, the rheology of large, thick wedges is much more complex than linear elasticity: the bulk rheology of thick wedges is most likely to be power-law steady-state creep (as argued later in this chapter). Second, the spatial distribution of normal and thrust faults in the same wedge shows significant variations of deformation pattern in the longitudinal direction (compressive at the thin-skinned foreland end and extensional at the thickskinned hinterland end) while Yin's results only show compression-extension variations in the vertical direction and not in the longitudinal. 
Although it is only a semi-quantitative solution, Platt's model (Platt, 1986) is probably the most interesting attempt to understand the dynamic stability of orogenic wedges. After presenting detailed arguments about the complicated nature of the rheology of thick orogenic wedges, Platt concludes that their bulk rheology can be described by some general nonlinear relation $\dot{\varepsilon}=f(\sigma)$ between the strain rate, $\dot{\varepsilon}$, and some invariant of deviatoric stress, $\sigma$. Therefore, material will be unstable and flow (i.e., $\dot{\varepsilon} \neq 0$ ) if the deviatoric stress is not equal to zero. Based upon this concept, Platt derived a stability criterion for two-dimensional plane strain thick orogenic wedges ( $c f$. Figure 2.5)

$$
\tau_{b}=\rho g h \alpha-2 \tau_{x x} \theta-2 \frac{\partial \tau_{x x}}{\partial x} h
$$

where $\theta(=\alpha+\beta)$ is the taper of the wedge and $\tau_{x x}=\frac{1}{h} \int_{0}^{h} \sigma_{x x}^{\prime} d y$ is the depth-averaged longitudinal deviatoric stress ( $h$ is the local thickness of the wedge and the coordinate system is as shown in Figure 2.5). All other parameters in equation (2.7) (e.g., $\rho, g, h, \alpha$ and $\tau_{b}$ ) are identical to those used in previous equations. Equation (2.7) states that the shear traction at the base of the wedge is balanced by three terms. The first is the familiar gravitational term due to the surface slope. The second is the result of the depth-averaged longitudinal deviatoric stress acting in a tapered wedge: because of the progressive change in cross-sectional area, this produces a net longitudinal force. The third term results from the gradient $\partial \tau_{x x} / \partial x$ in the longitudinal stress, which also produces a net force in the longitudinal direction.

The condition for stability is that the longitudinal strain rate $\dot{\varepsilon}_{x x}=0$. For a viscous material with rheology of the type $\dot{\varepsilon}_{x x}=f\left(\tau_{x x}\right)$, this implies that both $\tau_{x x}$ and $\partial \tau_{x x} / \partial x$ are zero. Consequently, equation (2.7) reduces to a stability criterion identical to that in the gravity spreading model (see equation (2.2)) 


$$
\alpha=\frac{\tau_{b}}{\rho g h}
$$

The role of surface slope $\alpha$ to maintain a critical state is apparent from equation (2.7). A value of $\alpha$ that is too small leads to horizontal compression and shortening in the wedge, while a value of $\alpha$ that is too large leads to extension of the wedge. If $\alpha$ is too small, the gravitational term in equation (2.7) is too small, so that either or both terms in $\tau_{x x}$ must be negative. Because $\tau_{x x}$ is likely to be zero or negative (compressional) at the front of the wedge, a negative $\partial \tau_{x x} / \partial x$ leads to a negative $\tau_{x x}$ throughout the wedge. Conversely, if $\alpha$ is too large, equation (2.7) requires that one or both terms in $\tau_{x x}$ be positive. The depth-averaged deviatoric stress towards the wedge front is limited by the strength of the material, and positive values of $\partial \tau_{x x} / \partial x$ will tend to lead to positive values of $\tau_{x x}$ away from the front of the wedge, resulting in extension and thereby reducing $\alpha$. The surface slope of wedge is also affected by material fluxes at the front, basement and surface of the wedge. Frontal accretion tends to lengthen the wedge, and consequently $\alpha$ will be low in the frontal region, which will therefore be in compression. Underplating, on the other hand, increases the surface slope $\alpha$ at the back of the wedge and consequently induces extension in the wedge ( $c f$. Platt, 1986).

Two factors have limited further applications of Platt's model:

(1) The depth-averaged longitudinal deviatoric stress $\tau_{x x}$ is a good approximation to the state of stress when the thickness of wedge is not too large (and therefore the longitudinal deviatoric stress $\sigma_{x x}^{\prime}$ does not vary significantly in the $y$-direction, that is, $\sigma_{x x}^{\prime}$ does not change much between the base $y=0$ and the surface $y=h$ ). For the thickskinned part of a wedge, it is likely that the state of stress will be quite different at various depths, implying that a depth-averaged parameter such as $\tau_{x x}$ may not be an acceptable approximation for the analysis of the effects of a change in topographic slope on the internal deformation of the wedge. 
(2) Because the bulk rheology of the wedge is left unspecified in Platt's model, it is impossible to make quantitative predictions from equation (2.7) on the dynamics of the wedge, including stress and velocity fields which affects the exhumation of high-pressure metamorphic rocks often observed in thick orogenic wedges.

The need for a quantitative description capable of accommodating the nonlinear nature of the bulk rheology of thick-skinned wedges has lead to a search for solutions derived in other disciplines for materials with nonlinear rheologies. One such solution which is of great interest to the dynamical analysis of orogenic wedges is a classic glacier flow solution (Nye, 1957). There are basically two reasons making this classic solution attractive: (1) Despite some important differences, there are obvious similarities between the movement of glaciers and ice sheets and that of nappes and thrust sheets (Elliott, 1976). All are wide bodies that are displaced for large distances over subhorizontal surfaces. In being displaced they become internally deformed and high shear strains are developed at the their bases (Hudleston, 1992); (2) Nye's solution is for the flow of an ice sheet with power-law bulk rheology, formally analogous to the most commonly observed high-temperature rheology of rocks. The formulation does not specify a priori the stress exponent $n$ for the sheet material, so it is sufficiently general to accommodate a wide range of deformation patterns which might be applicable to thick orogenic wedges: linear Newtonian flow $(n=1)$, perfectly plasticity $(n \rightarrow \infty)$, and power-law steady-state creep $(n=2 \sim 4)$.

In Nye's treatment, a uniform sheet with planar and parallel top and bottom surfaces flows in response to surface forces acting at its trailing edge and body forces due to its sloping upper surface (Figure 2.7). The solution is based on the following assumptions: (1) the material is incompressible and flows in a plane strain pattern; (2) stress and strain rate are linked by a power-law relationship; (3) the stress tensor is function of depth only (i.e., no longitudinal variations); (4) the vertical velocity component $v$ at any given depth 


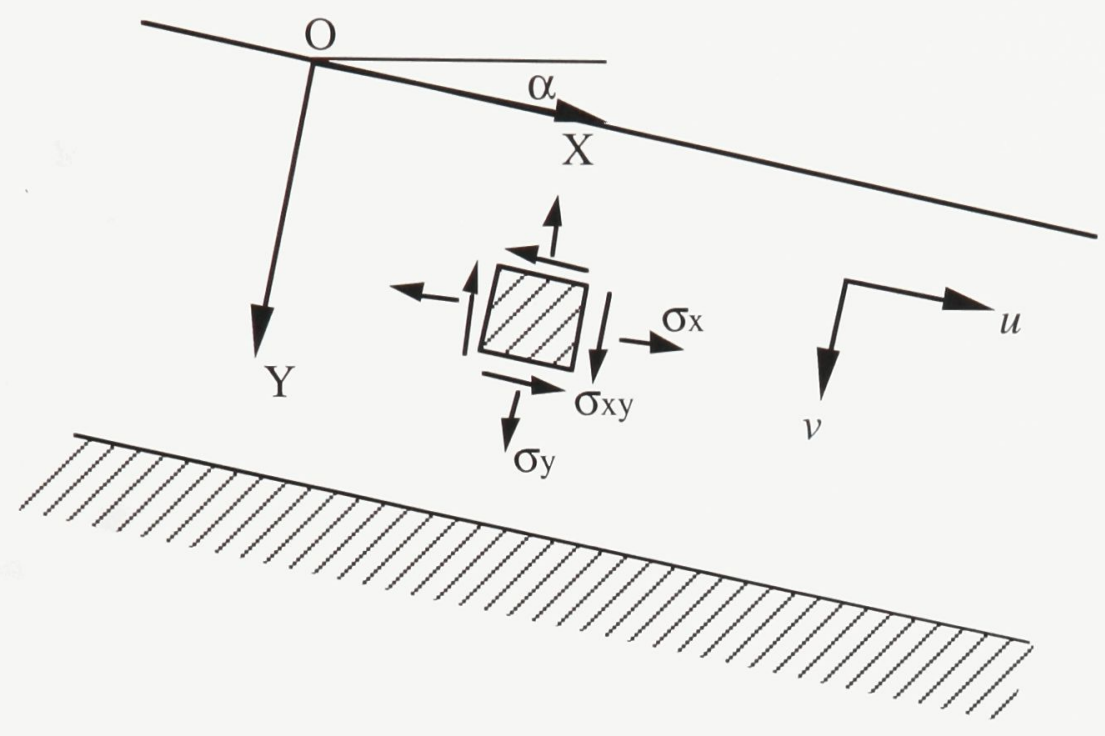

Figure 2.7 Notation and positive sense of stress components in the ice sheet model (from Nye 1957). 
does not vary with longitudinal position, that is, $\partial v / \partial x=0$. In other words, Nye's is a one-dimensional solution for power-law rheology.

A detailed discussion of Nye's solution for stress, strain rate and velocity will be found in Chapter 5. Shown in the followings are some depth-dependent profiles of dimensionless stress, velocity and displacement from Nye's solutions for a block with power-law relation between strain rate $\dot{\varepsilon}$ and corresponding stress deviatoric component $\sigma^{\prime}$ (i.e., $\left.\dot{\varepsilon}=\left(\sigma^{\prime} / A\right)^{n}\right)$. Figure 2.8 a shows the depth distribution of the second invariant of stress for sheets with different power-law stress exponent $n$. If parameter $A$ remains unchanged, the stress distributions for Newtonian viscous $(n=1)$ and almost plastic $(n=10)$ sheets bracket the range of stress intensity with depth. Velocity profiles for laminar flows with different power-law stress exponents $n$ are plotted in Figure 2.8b. The effect of $n$ on the deformation of sheets with laminar flow can also be presented in terms of displacement profiles as given in Figure 2.8c.

Nye's solution is applicable to sheets with uniform shortening or extending flows. It is not applicable, however, to sheets with a continuous variation of longitudinal strain rate, because of its one-dimensional assumption for stress which requires the longitudinal strain rate to be constant.

All the models mentioned above give analytical solutions. Numerical models have also been proposed to understand the complexities of foreland thrust belts and orogenic wedges. Some numerical models are briefly reviewed here.

Critical taper models describe wedges in a state of dynamic equilibrium, but do not explain how wedges deform internally to gain or regain their critical taper after equilibrium is disturbed by a change in boundary conditions. Willett (1992) presents a finite-element model of Coulomb wedges in order to simulate the growth of accretionary prisms and foreland fold and thrust belts. The constitutive behaviour adopted in this 

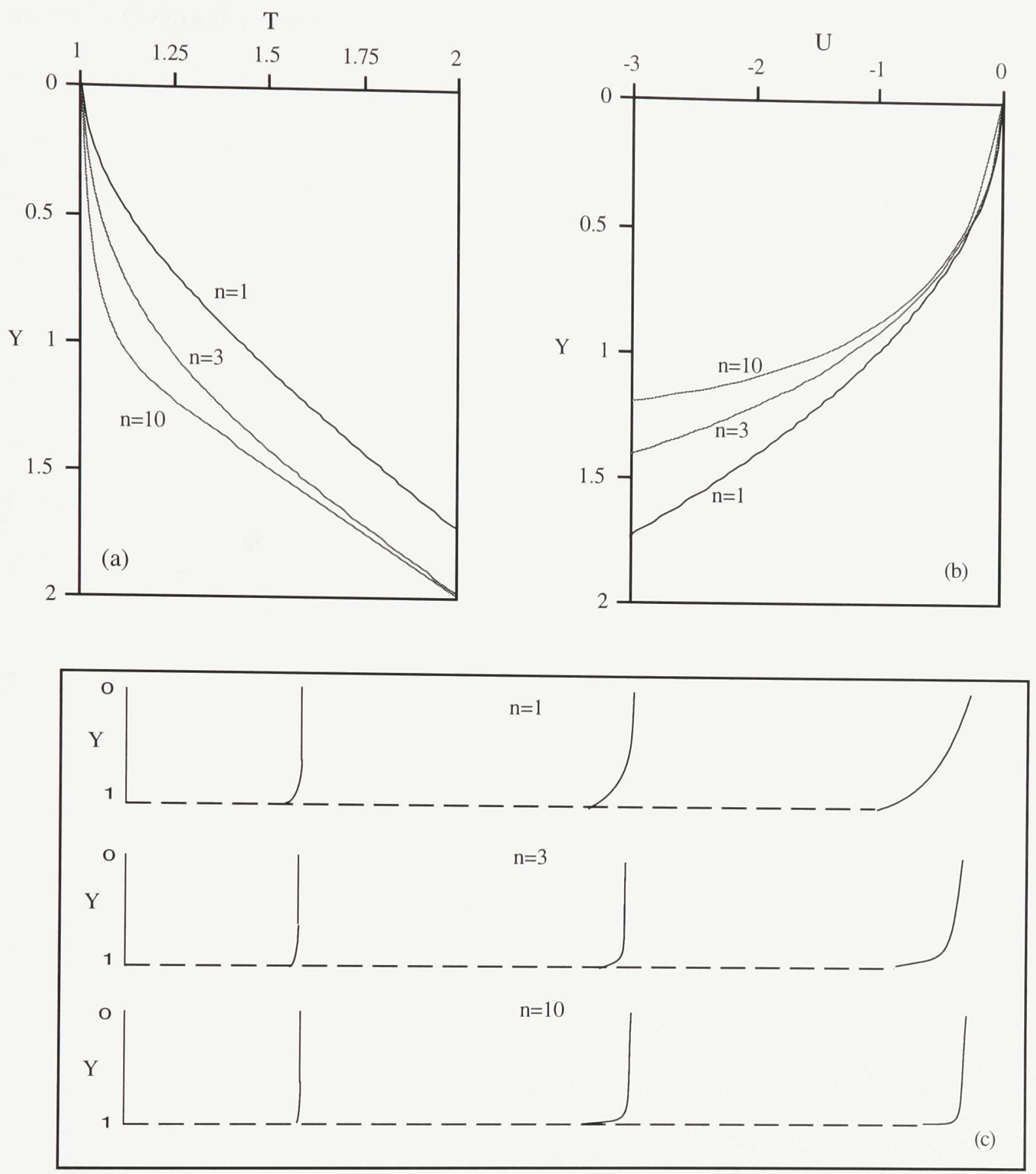

Figure 2.8 Depth distributions of dimensionless stress, velocity, and displacement from Nye's one-dimensional theory for different stress exponent $n$. (a) Second invariant of stress tensor (T); (b) longitudinal velocity (U); (c) longitudinal displacement for laminar flows (each sequence of curves from left to right represents the successive shapes taken by initially straight lines ; Y is dimensionless depth) ((a) and (b) from Nye, 1957; (c) from Wojtal, 1992). 
model is Coulomb plastic rheology, a variation of perfectly plastic rheology in which the von Mises yield strength is replaced by a scalar parameter derived from the non-cohesive Coulomb frictional criterion. The model demonstrates the self-similar growth of the critical Coulomb wedge and investigates the kinematics of wedges growing by frontal accretion. Changes in boundary conditions (e.g., a change in the basal friction) change the wedge geometry and thus may permit out of sequence thrusting (increase in basal friction) or extensional collapse of parts of the wedge while in compression (decrease in basal friction). This numerical model provides a good quantitative explanation for the formation of syn-convergence extension, even though the bulk rheology adopted is not very appropriate for thick-skinned orogenic wedges.

Beaumont et al. (1992a) describe a finite-element model of the evolution of the Southern Alps of New Zealand. In this model a rigid-plastic rheology is used to model brittle behaviour for the major part of the orogen and plastic-viscous rheology for the shear zone at the bottom of the orogen. This model incorporates tectonic and erosional data, and demonstrates that orogenic structure and metamorphic style may be controlled by the fluvial erosion and climate on the western flank of Southern Alps.

A similar finite-element model was used by Brown et al. (1993) to study the formation of coeval conjugate folds and thrusts observed in the southern Canadian Cordillera (the 'Selkirk fan structure'). This model demonstrates that a uniform Coulomb rheology layer deforming under basal boundary conditions of asymmetric detachment and underthrusting of the underlying substratum develops a structural fan over the leading edge of the detachment. The deforming layer has a cohesionless Coulomb yield criterion followed by a viscous post-yield flow. Results from this numerical model are similar to a sandbox experiment performed by Malavieille (1984). Similar mechanical models have also been applied to the analysis of other compressional orogens (Beaumont et al., 1992b; Willett et al., 1993; Beaumont and Quinlan, 1994). 
Recent developments in both numerical and analog models of orogenic wedges are reviewed in detail in McClay (1992).

\subsection{Rheology of orogenic wedges}

The fundamental difference between thin-skinned and thick-skinned orogenic wedges lies in the rheology: the rock material of supracrustal thrust belts and accretionary prisms is near the surface and consequently behaves in an elastic and brittle (frictional) fashion, while thick-skinned orogenic wedges with thickness large enough to induce metamorphism are unlikely to exhibit either plastic or Coulomb-type elastic-brittle rheologies throughout their volumes. The rheology applicable to the deeper parts of thickskinned orogenic wedges is most likely to be power-law steady-state creep.

From the rheological viewpoint, the following observations can be made:

(1) Below depths of about $10-20 \mathrm{~km}$ in the wedge, the material is probably capable of deforming at very low deviatoric stresses because of (a) the importance of pressuresolution processes in deforming metasedimentary rocks (at temperature between 450 and $800 \mathrm{~K}$ ), and (b) several softening mechanisms that are likely to act cooperatively at depth. Increasing temperature with depth reduces effective viscosities for all ductile deformation mechanisms, including the most common power-law creep (controlled by dislocation recovery). Metamorphic dehydration reactions in clay-rich sediments yield abundant water which reduces plastic yield strength in quartz by an order of magnitude (Blacic, 1975), facilitates diffusional creep (Rutter, 1976, 1983), favours subcritical corrosion cracking (Atkinson, 1980), and lowers the stress needed for brittle fracture (Etheridge, 1983). The evidence suggests that rocks at temperatures in the range of $600-800 \mathrm{~K}$ can deform at significant rates $\left(\geq 10^{-14} / \mathrm{sec}\right)$ under differential stresses of $10 \mathrm{MPa}$ (Rutter, 1976). 
(2) If the wedge is subjected to higher stresses, deformation will not become unconstrained (as in a perfect plastic or Coulomb material) but will simply occur at a higher rate. This can be described as general (nonlinear) viscoelastic behavior, for which the concept of a discrete, long-term yield-strength is meaningless. The material will deform, although slowly, under any deviatoric stress, however low. On the other hand, the material can "support" high stresses because of its viscous resistance to a high strainrate.

Taking these considerations into account, a steady-state power-law creep rheology of the type

$$
\dot{\varepsilon}=A_{0} \sigma^{n} \exp \left(-\frac{Q}{R T}\right)
$$

is adopted in this thesis as a first approximation to study the stress and deformation in orogenic wedges. In equation (2.9), $A_{0}$ is an empirically determined 'constant', $n$ is the stress exponent, $Q$ is the activation energy, $R$ is the universal gas constant, and $T$ is absolute temperature. Equation (2.9) fits very well the empirical steady-state creep for most materials at absolute temperatures above one-third to one-half of the melting point (Ranalli, 1995). The advantage of using a power-law constitutive equation such as (2.9) is that a broad spectrum of deformation processes can be formulated by using the same formal equation. For example, $n=1$ in equation (2.9) represents a linear Newtonian viscous flow while a very large value of $n$ simulates a plastic material. Therefore, the rheology of an orogenic wedge can be formulated by a uniform equation even though the bulk rheology of the wedge varies at different depths. On the other hand, the nonlinear nature of power-law creep rheology $(n>1)$ makes analytical solutions very difficult to find. 
Another major hurdle in devising quantitative models for orogenic wedges (both thinand thick- skinned) is that the longitudinal deformation pattern in a wedge often varies significantly from the thin-skinned foreland to the thick-skinned hinterland. Large-scale extension in compressional orogens, not only post-compressional but also syncompressional, is common (Platt, 1986; Dewey, 1988; Twiss and Moores, 1992; Burchfiel et al. 1992; Gottschalk and Oldow, 1988; Oldow et al., 1993; Yin, 1993). Associated closely with the syn-convergent extension at the rear of an orogenic wedge is the exhumation of large volumes of high-pressure metamorphic rocks.

As mentioned above, extensional structures may develop not only concurrently with compressive tectonic processes but also after the convergent process ends. This postshortening extension can be caused by lateral variations in thickness and/or density within plates and by lithospheric delamination (Dalmayrac and Molnar, 1981; Artyushkov, 1983; Molnar and Lyon-Caen, 1988; Bott, 1993; Ranalli, 1995).

In conclusion, the popular 'critical taper' theories (including both plastic and Coulomb-type frictional models) are not directly applicable to thick-skinned orogenic wedges with respect to both rheology and style of deformation. In terms of bulk rheology, orogenic wedges are dominated by steady-state power-law creep instead of simple homogeneous frictional brittle deformation. In terms of deformation style, orogenic wedges often show longitudinal differences between the compressive front (characterized by significant thrust faults) and the extensional rear. Elastic models (Yin, 1993) are useful to understand the mechanical conditions for the initiation of normal and/or thrust faults in thrust wedges, but the constitutive equation adopted prevents their application to thickskinned wedges. Nye's (1957) classic theory for glaciers is able to accommodate nonlinear (power-law) rheology, but is one-dimensional (quantities vary only in the vertical direction), and consequently is not capable of simulating the variation of deformation in the longitudinal direction along a wedge. 
Platt's $(1986,1993)$ recognition of the very complicated nonlinear nature of rheology in thick-skinned orogenic wedges and his explanation of dynamic stability in terms of depth-averaged longitudinal deviatoric normal stress (which is related to the longitudinal strain rate by an unspecified creep law) is probably the most interesting and promising attempt to understand the processes at work during the evolution of orogenic belts (e.g., frontal accretion, underplating, exhumation of high-pressure metamorphic rocks, and the coeval development of extensional and compressional tectonics). Platt's model, however, is qualitative and therefore does not allow computation of stresses and deformation fields.

It follows from the previous considerations that a quantitative description of thick orogenic wedges in terms of a general nonlinear bulk rheology would be very useful. However, it is also obvious that the complexities of real wedges effectively preclude their detailed modelling. However, a simplified (e.g., homogeneous, isotropic) model would be an important step forward. The major difficulty in obtaining an explicit solution for stress $(\sigma)$, strain rate $(\dot{\varepsilon})$, and velocity fields $(v)$, from the mathematical point of view, is the nonlinear power-law relation between the deviatoric stresses and corresponding strain rates. The search for such an analytical solution is the main motivation of this thesis, whose objective is to provide closed-form, analytical solutions simulating the stress and deformation of orogenic wedges at variable scales. On the scale of a single thrust, a static elastic approach, by the use of the Airy stress function, is used to analyze the development of an overthrust fault and the movement of individual thrust sheets. On the scale of a whole orogenic wedge, a generalized steady-state power-law rheology is incorporated in the modelling, and an explicit analytical solution for stress, strain rate and velocity fields in orogenic wedges is derived. 


\section{Chapter 3}

\section{Stresses in An Overthrust Sheet and Propagation of Thrusting Along Its Base(1)}

\subsection{Introduction}

Orogenic wedge modelling can basically be done on two different scales: on the scale of the whole wedge, where the material deforms as a single, mechanically continuous unit; on the scale of individual thrust sheets, where deformation occurs mainly by relative displacement along thrust faults. In this chapter, attention is focused on the stress and deformation within a single wedge-shaped thrust block and along its base. In particular, an effort is made to improve our understanding of one of the most debated questions in modelling the movement of thrust sheets, that is, the so-called mechanical paradox of overthrusting .

The mechanical paradox of overthrusting originates with the obvious difference between the geological observation of large overthrust sheets and the theoretical estimation of the maximum length of an overthrust block that may be displaced along a thrust fault. This theoretical value is determined by using the Coulomb-Navier criterion to compare the strength of the rocks with the stress required to overcome the total frictional resistance to slip ( $c f$. Smoluchowski, 1909). In his analysis, Smoluchowski took the coefficient of friction $\mu_{0}=0.15$ (equal to that of iron sliding on iron) and inferred that a 100-km-long block would require a stress far greater than any rock would sustain.

(1) This chapter, with minor modifications, has been published as:

Liu, J. Y. and Ranalli, G. (1992) Stress in an overthrust sheet and propagation of thrusting: an Airy stress function solution. Tectonics, 11, 549-559. 
Since a value of 0.15 is less than the coefficient of friction for most rocks ( $c f$. Jaeger and Cook, 1969; Byerlee, 1978; Ranalli, 1995), a larger but more unrealistic stress will result if the coefficient of friction for rocks is taken into consideration (see equation 2.1).

To overcome this conceptual difficulty, a variety of attempts have been made (for a brief review, see Suppe, 1985). For instance, Hubbert and Rubey (1959) considered the possible existence of a layer of high pore fluid pressure to reduce the frictional resistance to sliding. The importance of the cohesive strength of the rocks was further studied by Birch (1961) and Hsü (1969). On the basis of the observation that the total displacement due to earthquake faulting and fault creep clearly varies from place to place over a thrust fault, Price $(1973 b, 1988)$ argued that in all mechanical models of overthrusting there is an unstated and untenable assumption that displacement occurs simultaneously over the entire fault surface. This assumption is demonstrably fallacious when applied to seismic slip (De Bremaecker, 1987). Both Price and De Bremaecker concluded that, instead of simultaneous displacement along the thrust fault, a "dislocation-type motion" may be a more reasonable mechanism able to overcome the mechanical paradox. The displacement is achieved by slip episodes that nucleate at a given point on the thrust surface and propagate with finite velocity. The total displacement of the thrust sheet is therefore the resultant of several small consecutive slips, each requiring a force much less than the estimations based on the assumption of simultaneous slippage.

One of the implications of the "dislocation" model is that the state of stress varies from place to place along the thrust, contrary to what is assumed in the "classical" models. Naturally the frictional strength may vary with position too, but in this chapter the focus is on variations of the state of stress. Its variation on the thrust surface is caused by two reasons: statically, the shear and normal stresses are not necessarily constant but depend on the external force system and the model geometry; dynamically, the propagation of the dislocation modifies the stress field ( $c f$. Eshelby, 1973; Savage, 1980). 
The static inhomogeneous stress state plays a key role in triggering the "dislocation" motion of the entire thrust fault, since slip will occur only where the strength is exceeded.

This chapter investigates the static "initial" conditions of thrusting, that is, the problem of obtaining stress distributions on the thrust plane that are compatible with the initiation of progressive slip. A thrust sheet is modeled as a two-dimensional wedgeshaped block of elastic-brittle rocks, with compressive boundary conditions on both rear and front edges (see Figure 3.1). The assumption of linear elasticity (at stresses below the strength) is of course an approximation, but it is not unrealistic at shallow crustal levels ( $\leq 10-15 \mathrm{~km}$; see Ranalli, 1995). The model, consequently, applies to thin-skinned thrust sheets but not necessarily to thick (basement) thrusts. The truncated wedge geometry allows derivation for both rectangular and triangular shapes as particular cases. The contact plane between the thrust sheet and the underlying basement is a surface along which displacement may occur. In this sense, the model is different from both the Coulomb wedge model (see, for example, Davis et al., 1983; Dahlen and Suppe, 1988), which considers failure within the wedge, and models where the bottom of the block is taken as fixed or coupled with the underlying basement (see, for example, Hafner, 1951; Mandl and Shippam, 1981; Mandl, 1988; Yin, 1989). The problem is solved by means of Airy stress functions to derive the stress state within the wedge, from which the state of stress along the dipping lower boundary of the sheet can be determined. Airy stress functions have been previously applied to elastic wedges by Yin (1986, 1988, 1993). In the present approach, however, the state of stress on the basal thrust is not predetermined by a frictional law but is a consequence of boundary conditions and of the shape of the wedge. In other words, the solution provided in this chapter is for a model with “incompletely-prescribed boundary conditions" ( $c f$. Timoshenko and Goodier, 1970).

The occurrence of displacement along a failure plane is governed by the CoulombNavier fracture criterion ( $c f$. Jaeger and Cook, 1969) 


$$
\tau_{n}=S_{0}+\mu_{0} \sigma_{n}
$$

in which there are two parameters (cohesive strength $S_{0}$ and coefficient of friction $\mu_{0}$ ) representing the mechanical properties of the rock and two components of stress (normal stress $\sigma_{n}$, corrected for pore fluid pressure if necessary, and shear stress $\tau_{n}$ ) describing the state of stress along the failure surface. The solution presented in this chapter shows that the state of stress along the basal thrust fault varies with position if (1) the slopes of the upper and lower boundaries of the thrust sheet are not zero, and/or (2) the horizontal compressional driving forces exerted on the vertical boundaries vary nonlinearly with depth rather than linearly as assumed by Hafner (1951), Hubbert and Rubey (1959), Mandl (1988), and Yin (1989). Although the second contribution is hypothetical, the first is certainly satisfied in most thrust sheets. The results provide some theoretical support for the "dislocation" model for overthrust faults, despite being obtained from simple static considerations.

\subsection{State of stress within the thrust sheet}

The geometry of the model is shown in Figure 3.1. The origin of $x$ and $y$ coordinates is set at the upper left corner of the block, whose length and rear thickness are $L$ and $H$. The slopes of the upper free surface and of the lower thrust fault are $\alpha$ and $\beta$, respectively (both positive when the thickness of the wedge decreases toward the front).

Engineering stress sign convention is adopted in this thesis and therefore for the twodimensional case $\sigma_{1}$ and $\sigma_{2}$ represent the minimum and maximum compression, respectively. 


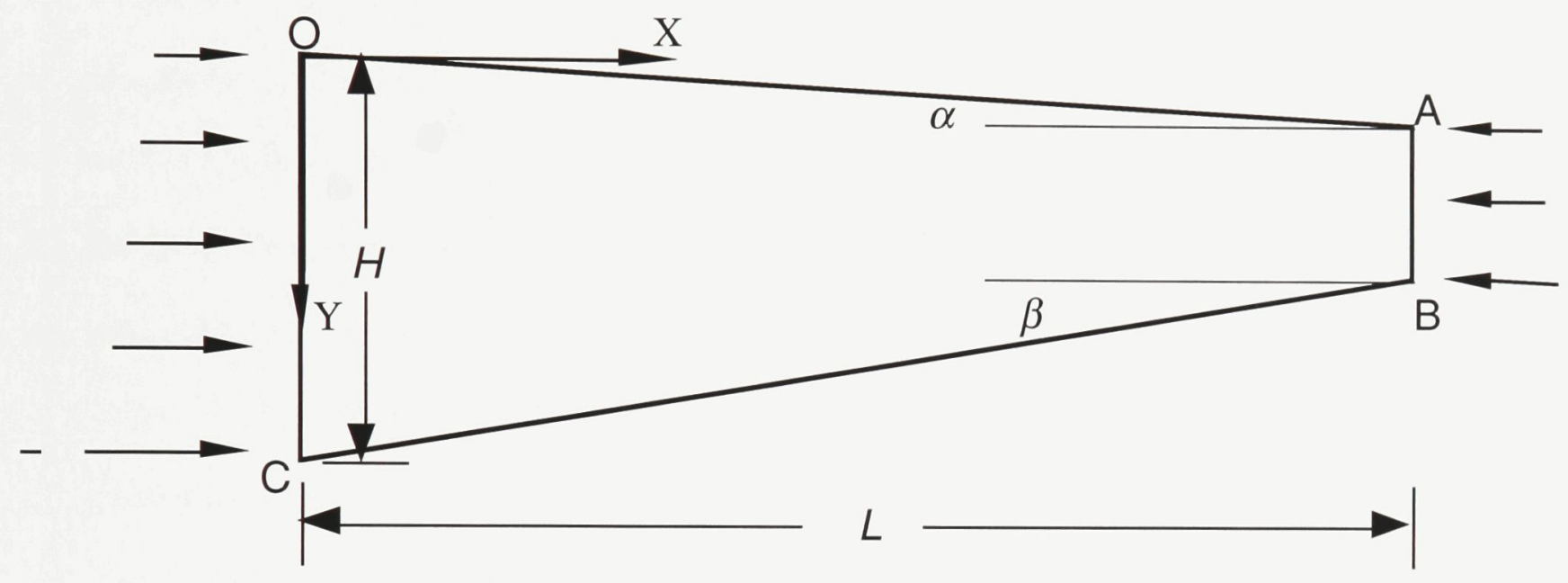

Figure 3.1 Geometry of wedge-shaped thrust sheet under horizontal compression. The coordinate origin is set at the upper left corner. $L$ and $H$ are the horizontal length and rear thickness of the block; $\alpha$ and $\beta$ the slopes of the upper and lower boundaries. 
The horizontal compressional forces on both rear and front vertical edges are taken in the general case to be parabolic functions of depth (which includes linearity as a special case), so that the boundary conditions are

$$
\begin{aligned}
& F_{x}^{O C}=a y^{2}+b y+c \\
& F_{x}^{A B}=a_{1} y^{2}+b_{1} y+c_{1} \\
& F_{x}^{O A}=F_{y}^{O A}=0
\end{aligned}
$$

where the superscripts $\mathrm{OC}, \mathrm{AB}$, and $\mathrm{OA}$ refer to the boundary planes on which the forces are applied. The third equation represents conditions on the free surface, where $y=n x$, with $n=\tan \alpha$. The parameters $a, a_{1}, b, b_{1}, c$, and $c_{1}$ are taken as positive when acting in the positive $x$ direction. The force (and consequent stress) at the base of the block is not prescribed in advance but is a consequence of the horizontal boundary forces and the shape of the block. According to Kirchhoff's theorem ( $c f$. Fung, 1965), the solution is therefore not unique, since the boundary conditions are not fully prescribed. However, it is acceptable, in the sense that it satisfies compatibility of strain (through the biharmonic equation) and the equilibrium conditions. (Solutions for incomplete boundary conditions are not uncommon in elastostatics; see, for example, Fung (1965) and Timoshenko and Goodier (1970).)

The relation between boundary forces and internal stress components can be expressed in terms of the Airy stress function ( $c f$. Fung, 1965).

$$
\begin{aligned}
& F_{x}=\frac{\partial^{2} \Phi}{\partial y^{2}} \cos \theta-\frac{\partial^{2} \Phi}{\partial x \partial y} \sin \theta \\
& F_{y}=-\frac{\partial^{2} \Phi}{\partial x \partial y} \cos \theta+\left(\frac{\partial^{2} \Phi}{\partial x^{2}}-\rho g y\right) \sin \theta
\end{aligned}
$$

where $F_{x}, F_{y}$ are the boundary forces applied in the $x$ and $y$ directions, respectively; $\Phi(x, y)$ is the stress function; $\theta$ is the angle between the normal to a given boundary and 
the positive $x$ direction, and $\rho$ and $g$ are density and gravity, respectively. The stress function is related to the stress components as

$$
\begin{aligned}
& \sigma_{x x}=\frac{\partial^{2} \Phi}{\partial y^{2}} \\
& \sigma_{y y}=\frac{\partial^{2} \Phi}{\partial x^{2}}-\rho g y \\
& \sigma_{x y}=-\frac{\partial^{2} \Phi}{\partial x \partial y}
\end{aligned}
$$

where $\sigma_{x x}, \sigma_{y y}$, and $\sigma_{x y}$ are the three stress components.

Because of the general understanding about the state of stress within the upper crust and the stress boundary conditions applied at the front and at the back of the thrust sheet, it is not likely that the highest order of the polynominal expression for the stress field, with respect to $x$ or $y$, will exceed three. Without losing generality, the stress function $\Phi(x, y)$ can be assumed to be of the form

$$
\begin{aligned}
\Phi(x, y) & =\sum_{i=1}^{4} k_{i} x^{i+1}+y \sum_{i=5}^{9} k_{i} x^{i-4}+y^{2} \sum_{i=10}^{15} k_{i} x^{i-10}+y^{3} \sum_{i=6}^{21} k_{i} x^{i-16} \\
& +y^{4} \sum_{i=22}^{27} k_{i} x^{i-22}+y^{5} \sum_{i=28}^{33} k_{i} x^{i-28}
\end{aligned}
$$

where $k_{i}(i=1$ to 33$)$ are arbitrary constants to be determined from the boundary conditions and the requirement that $\nabla^{4} \Phi \equiv\left(\partial^{4} / \partial x^{4}+2 \partial^{4} / \partial x^{2} \partial y^{2}+\partial^{4} / \partial y^{4}\right) \Phi=0$. The latter leads to

$$
\begin{aligned}
& 3 k_{3}+k_{12}+3 k_{22}=0 \\
& 5 k_{4}+k_{13}+k_{23}=0 \\
& k_{8}+k_{18}+5 k_{28}=0 \\
& 5 k_{9}+3 k_{19}+5 k_{29}=0 \\
& k_{14}=k_{15}=k_{20}=k_{21}=k_{24}=k_{25}=k_{26}=k_{27}=k_{30}=k_{31}=k_{32}=k_{33}=0
\end{aligned}
$$


By combining equations (3.2), (3.3), and (3.4), the condition for traction-free upper surface can be expressed as

$$
\begin{aligned}
& 5 n^{4} k_{29}+3 n^{2} k_{19}+k_{9}=0 \\
& 5 n^{4} k_{28}+4 n^{3} k_{23}+3 n^{2} k_{18}+2 n k_{13}+k_{8}=0 \\
& 4 n^{3} k_{22}+3 n^{2} k_{17}+2 n k_{12}+k_{7}=0 \\
& 3 n^{2} k_{16}+2 n k_{11}+k_{6}=0 \\
& 2 n k_{10}+k_{5}=0
\end{aligned}
$$

and

$$
\begin{aligned}
& n^{4} k_{29}+3 n^{2} k_{19}+5 k_{9}=0 \\
& n^{4} k_{23}+2 n^{3} k_{18}+3 n^{2} k_{13}+4 n k_{8}+5 k_{4}=0 \\
& n^{3} k_{17}+2 n^{2} k_{12}+3 n k_{7}+4 k_{3}=0 \\
& n^{2} k_{11}+2 n k_{6}+3 k_{2}=\frac{n}{2} \rho g \\
& n k_{5}+2 k_{1}=0
\end{aligned}
$$

Equations (3.6a, b), combined with equations (3.2) and (3.3), lead to the determination of the remaining arbitrary constants

$$
\begin{aligned}
& k_{1}=-\frac{n^{2}}{2} c \\
& k_{2}=\frac{n}{6} \rho g-\frac{n^{2} t_{3}}{4 t_{1}} a L+\frac{n^{2} t_{6}}{12 t_{1} t_{4}}\left(a+a_{1}\right) L-\frac{n^{3}}{3} b+\frac{n^{5}}{t_{1}}\left(b+b_{1}\right)+\frac{n^{2}}{2 L}\left(c+c_{1}\right) \\
& k_{3}=\frac{n^{2} t_{2}}{4 t_{1}} a+\frac{n^{4} t_{5}}{3 t_{1} t_{4}}\left(a+a_{1}\right)+\frac{n^{3}}{3 t_{1}} \frac{b+b_{1}}{L} \\
& k_{4}=-\frac{n^{2} t_{2}^{2}}{12 t_{4}} \frac{a+a_{1}}{L} \\
& k_{5}=n c \\
& k_{6}=\frac{n t_{3}}{2 t_{1}} a L-\frac{n t_{6}}{6 t_{1} t_{4}}\left(a+a_{1}\right) L+\frac{n^{2}}{2} b-\frac{2 n^{4}}{t_{1}}\left(b+b_{1}\right)-\frac{n}{L}\left(c+c_{1}\right) \\
& k_{7}=-\frac{n\left(2 t_{2}+t_{3}\right)}{6 t_{1}} a-\frac{n^{3} t_{2} t_{5}}{2 t_{1} t_{4}}\left(a+a_{1}\right)-\frac{n^{2} t_{2}}{2 t_{1}} \frac{b+b_{1}}{L}
\end{aligned}
$$




$$
\begin{aligned}
& k_{8}=\frac{n t_{5}}{6 t_{4}} \frac{a+a_{1}}{L} \\
& k_{9}=0 \\
& k_{10}=-\frac{c}{2} \\
& k_{11}=-\frac{t_{3}}{4 t_{1}} a L+\frac{t_{6}}{12 t_{1} t_{4}}\left(a+a_{1}\right) L+\frac{n^{3}}{t_{1}}\left(b+b_{1}\right)+\frac{c+c_{1}}{2 L} \\
& k_{12}=-\frac{t_{3}}{4 t_{1}} a-\frac{n^{4} t_{5}}{t_{1} t_{4}}\left(a+a_{1}\right)-\frac{n^{3}}{t_{1}} \frac{b+b_{1}}{L} \\
& k_{13}=-\frac{t_{2} t_{5}+8 n^{4}}{12 t_{4}} \frac{a+a_{1}}{L} \\
& k_{16}=-\frac{b}{6} \\
& k_{17}=\frac{n t_{5}}{6 t_{4}}\left(a+a_{1}\right)+\frac{b+b_{1}}{6 L} \\
& k_{18}=-\frac{n t_{5}}{6 t_{4}} \frac{a+a_{1}}{L} \\
& k_{19}=0 \\
& k_{22}=-\frac{a}{12} \\
& k_{23}=-\frac{a+a_{1}}{12 L} \\
& k_{28}=k_{29}=0 \\
& k_{1}
\end{aligned}
$$

In equations (3.7), $t_{1}=1+3 n^{2}, \quad t_{2}=1-n^{2}, \quad t_{3}=1+3 n^{4}, \quad t_{4}=1+2 n^{2}+5 n^{4}$, $t_{5}=1-2 n^{2}+5 n^{4}$ and $t_{6}=1+18 n^{4}+16 n^{6}+45 n^{8}$.

Recalling equations (3.3), the external forces on rear and front vertical edges and the resulting force on the bottom surface can be written as

$$
\begin{aligned}
F_{x}^{O C} & =a y^{2}+b y+c \\
F_{y}^{O C} & =k_{5}+2 k_{11} y+3 k_{17} y^{2}+4 k_{23} y^{3} \\
F_{x}^{A B} & =a_{1} y^{2}+b_{1} y+c_{1} \\
F_{y}^{A B} & =-\left(k_{5}+2 L k_{6}+3 L^{2} k_{7}+4 L^{3} k_{8}\right)-2\left(k_{11}+2 L k_{12}+3 L^{2} k_{13}\right) y \\
& -3\left(k_{17}+2 L k_{18}\right) y^{2}-4 k_{23} y^{3} \\
F_{x}^{B C} & =M_{0}+M_{1} x+M_{2} x^{2}+M_{3} x^{3} \\
F_{y}^{B C} & =N_{0}+N_{1} x+N_{2} x^{2}+N_{3} x^{3}
\end{aligned}
$$


where $m=\tan \beta, w=\left(1+m^{2}\right)^{-1 / 2}$ and the parameters $M_{i}, N_{i}(i=0$ to 3$)$ are given by

$$
\begin{aligned}
M_{0}= & w\left(-k_{5}+2 m k_{10}-2 H k_{11}+6 H m k_{16}-3 H^{2} k_{17}+12 H^{2} m k_{22}-4 H^{3} k_{23}\right) \\
M_{1}= & 2 w\left(-k_{6}+2 m k_{11}-2 H k_{12}-3 m^{2} k_{16}+6 H m k_{17}-3 H^{2} k_{18}\right. \\
& \left.-12 H m^{2} k_{22}+12 H^{2} m k_{23}\right) \\
M_{2}= & 3 w\left(-k_{7}+2 m k_{12}-2 H k_{13}-3 m^{2} k_{17}+6 H m k_{18}+4 m^{3} k_{22}-12 H m^{2} k_{23}\right) \\
M_{3}= & 4 w\left(-k_{8}+2 m k_{13}-3 m^{2} k_{18}+4 m^{3} k_{23}\right) \\
N_{0}= & w\left(2 k_{1}-m k_{5}+2 H k_{6}-2 H m k_{11}+2 H^{2} k_{12}-3 H^{2} m k_{17}+2 H^{3} k_{18}\right. \\
& \left.-4 H^{3} m k_{23}-\rho g H\right) \\
N_{1}= & 2 w\left(3 k_{2}-2 m k_{6}+3 H k_{7}+m^{2} k_{11}-4 H m k_{12}+3 H^{2} k_{13}+2 H m^{2} k_{17}\right. \\
& \left.-6 H^{2} m k_{18}+6 H^{2} m^{2} k_{23}+\frac{m \rho g}{2}\right) \\
N_{2}= & 3 w\left(4 k_{3}-3 m k_{7}+4 H k_{8}+2 m^{2} k_{12}-6 H m k_{13}-m^{3} k_{17}+6 H m^{2} k_{18}-4 H m^{3} k_{23}\right) \\
N_{3}= & 4 w\left(5 k_{4}-4 m k_{8}+3 m^{2} k_{13}-2 m^{3} k_{18}+m^{4} k_{23}\right)
\end{aligned}
$$

Equations (3.8) can be proven to satisfy the resulting force and moment equilibria.

The constants $k_{i}$ can be subdivided into two sets, only one of which is dependent on the upper slope $n$ (see equation (3.7)). Consequently, the stresses can be written as

$$
\begin{aligned}
& \sigma_{x x}=\sigma_{x x}^{\prime}+\sigma_{x x}^{\prime \prime} \\
& \sigma_{y y}=\sigma_{y y}^{\prime}+\sigma_{y y}^{\prime \prime} \\
& \sigma_{x y}=\sigma_{x y}^{\prime}+\sigma_{x y}^{\prime \prime}
\end{aligned}
$$

where a prime denotes the components independent of the upper (and also the lower) slope (that is, the components of the state of stress in a rectangular block of length $L$ and thickness $H$ subject to the same horizontal external forces). The double prime denotes the components that are dependent on the slope $n$, i.e., the part of the stress field resulting from the effect of the wedge-shaped geometry.

A concise approximate expression for the stress solution can be obtained by neglecting individual terms that constitute $<1 \%$ of the value of any given $k_{i}$ in equation 
(3.7) for reasonable parameter ranges $\left(\alpha, \beta \leq 6^{\circ}\right.$, and $L / H \geq 10$; $c$. Boyer and Elliott, 1982; Davis et al., 1983; Suppe, 1985). The nonlinear parts are chosen in such way that shear stresses are realistic (i.e., $\tau_{n} \leq 50 \mathrm{MPa}$ ) and deviations of the vertical stress from lithostatic values are minor (see discussion at the end of this section).

Using the above simplifications and equation (3.3b), the stress components can be written as

$$
\begin{aligned}
& \sigma_{x x}^{\prime}=\frac{a+a_{1}}{6 L} x^{3}+\frac{a}{2} x^{2}+\left(\frac{c+c_{1}}{L}-\frac{2 a-a_{1}}{6} L\right) x+\frac{b+b_{1}}{L} x y+\frac{a+a_{1}}{L} x y^{2}-a y^{2}-b y-c \\
& \sigma_{y y}^{\prime}=-\frac{a+a_{1}}{2 L} x y^{2}+\frac{a}{2} y^{2}-\rho g y \\
& \sigma_{x y}^{\prime}=\frac{a+a_{1}}{2 L} x^{2} y-a x y-\frac{a+a_{1}}{3 L} y^{3}-\frac{b+b_{1}}{2 L} y^{2}-\left(\frac{c+c_{1}}{L}-\frac{2 a-a_{1}}{6} L\right) y
\end{aligned}
$$

and

$$
\begin{aligned}
\sigma_{x x}^{\prime \prime}= & n\left[-\frac{a+a_{1}}{L} x^{2} y+\left(a+a_{1}\right) x y\right] \\
\sigma_{y y}^{\prime \prime}= & n\left[2 \frac{a+a_{1}}{L} x^{2} y-3 a x y+\rho g x-\frac{a+a_{1}}{3 L} y^{3}-\left(2 \frac{c+c_{1}}{L}-\frac{2 a-a_{1}}{3} L\right) y\right] \\
& +n^{2}\left[-\frac{5\left(a+a_{1}\right)}{3 L} x^{3}+3 a x^{2}+\left(3 \frac{c+c_{1}}{L}-\frac{2 a-a_{1}}{2} L\right) x-3 \frac{b+b_{1}}{L} x y+b y-c\right] \\
& +n^{3} \frac{4\left(b+b_{1}\right)}{L} x^{2} \\
\sigma_{x y}^{\prime \prime}= & n\left[-\frac{2\left(a+a_{1}\right)}{3 L} x^{3}+\frac{3 a}{2} x^{2}+\left(2 \frac{c+c_{1}}{L}-\frac{2 a-a_{1}}{3} L\right) x+\frac{a+a_{1}}{L} x y^{2}-\frac{a+a_{1}}{2} y^{2}-c\right] \\
& +n^{2}\left[\frac{3\left(b+b_{1}\right)}{2 L} x^{2}-b x\right]
\end{aligned}
$$

Although the slope of the basal surface does not appear explicitly in the stress solution, the thickness of the frontal edge is determined by the slopes of both upper and 
lower boundaries, that is, $H^{\prime}=H-(n+m) L$, and the frontal buttressing force is in fact affected by the lower boundary geometry.

The case of a triangular wedge pushed from the rear can be derived from the general case for vanishing frontal edge thickness. In this case, $a_{1}=b_{1}=c_{1}=0$ and $(m+n) L=H$. Denoting the stresses in the triangular wedge by $\tau$ and adopting the same notation as in equations (3.10), the stress components are

$$
\begin{aligned}
& \tau_{x x}^{\prime}=\frac{a}{6 L} x^{3}+\frac{a}{2} x^{2}+\left(\frac{c}{L}-\frac{a L}{3}\right) x+\frac{b}{L} x y+\frac{a}{L} x y^{2}-a y^{2}-b y-c \\
& \tau_{y y}^{\prime}=-\frac{a}{2 L} x y^{2}+\frac{a}{2} y^{2}-\rho g y \\
& \tau_{x y}^{\prime}=\frac{a}{2 L} x^{2} y-a x y-\frac{a}{3 L} y^{3}-\frac{b}{2 L} y^{2}-\left(\frac{c}{L}-\frac{a L}{3}\right) y
\end{aligned}
$$

and

$$
\begin{aligned}
\tau_{x x}^{\prime \prime}= & n\left[-\frac{a}{L} x^{2} y+a x y\right] \\
\tau_{y y}^{\prime \prime}= & n\left[\frac{2 a}{L} x^{2} y-3 a x y+\rho g x-\frac{a}{3 L} y^{3}-\left(\frac{2 c}{L}-\frac{2 a L}{3}\right) y\right] \\
& +n^{2}\left[-\frac{5 a}{3 L} x^{3}+3 a x^{2}+\left(\frac{3 c}{L}-a L\right) x-\frac{3 b}{L} x y+b y-c\right]+n^{3} \frac{4 b}{L} x^{2} \\
\tau_{x y}^{\prime \prime}= & n\left[-\frac{2 a}{3 L} x^{3}+\frac{3 a}{2} x^{2}+\left(\frac{2 c}{L}-\frac{2 a L}{3}\right) x+\frac{a}{L} x y^{2}-\frac{a}{2} y^{2}-c\right]+n^{2}\left[\frac{3 b}{2 L} x^{2}-b x\right]
\end{aligned}
$$

This stress solution may be applied to the determination of the internal state of stress and the stress along the basal thrust in thin-skinned wedges of approximately triangular cross section.

The principal stress trajectories within the thrust sheet are plotted in Figure 3.2 for the parameters given in Tables 3.1 and 3.2. The trajectories of maximum shear stress are 
compatible with the upwardly concave secondary thrusts commonly observed in thrust belts.

Table 3.1 Values of model parameters for rectangular block with dimensions $L=10 \mathrm{~km}$, $H=1 \mathrm{~km}$ and density $\rho=2800 \mathrm{~kg} / \mathrm{m}^{3}$ subject to nonlinear boundary forces.

\begin{tabular}{ccccc} 
& \multicolumn{5}{c}{ Case $^{*}$} \\
\cline { 2 - 5 } Parameters & $\mathrm{A}$ & $\mathrm{B}$ & $\mathrm{C}$ & $\mathrm{D}$ \\
\hline$a\left(\mathrm{MPa} / \mathrm{km}^{2}\right)$ & -0.8 & -2.0 & -2.0 & 0.8 \\
$a_{1}\left(\mathrm{MPa} / \mathrm{km}^{2}\right)$ & 0 & 0 & 0 & 0 \\
$b(\mathrm{MPa} / \mathrm{km})$ & 32.9 & 32.9 & 35.7 & 35.7 \\
$b_{1}(\mathrm{MPa} / \mathrm{km})$ & -22.0 & -22.0 & -22.0 & -22.0 \\
$c(\mathrm{MPa})$ & 110 & 110 & 120 & 120 \\
$c_{1}(\mathrm{MPa})$ & 0 & 0 & 0 & 0 \\
\hline
\end{tabular}

* Cases A, B, C, and D refer to curves in Figures 3.4a, 3.4b, and 3.7a.

Table 3.2 Values of model parameters for wedge-shaped block with dimensions $L=10$ $\mathrm{km}, H=1 \mathrm{~km}$ subject to linear boundary forces

\begin{tabular}{ccccc}
\hline & \multicolumn{3}{c}{ Case $^{*}$} \\
\cline { 2 - 5 } Parameters & $\mathrm{A}$ & $\mathrm{B}$ & $\mathrm{C}$ & $\mathrm{D}$ \\
\hline$b(\mathrm{MPa} / \mathrm{km})$ & 32.9 & 32.9 & 35.7 & 35.7 \\
$b_{1}(\mathrm{MPa} / \mathrm{km})$ & -22.0 & -22.0 & -22.0 & -22.0 \\
$c(\mathrm{MPa})$ & 110 & 110 & 110 & 120 \\
$c_{1}(\mathrm{MPa})$ & 0 & 0 & 0 & 0 \\
$\alpha$ & $2^{\circ}$ & $2^{\circ}$ & $2^{\circ}$ & $2.5^{\circ}$ \\
$\beta$ & $1^{\circ}$ & $2^{\circ}$ & $2^{\circ}$ & $2^{\circ}$ \\
\hline
\end{tabular}

* Cases A, B, C, and D refer to curves in Figures 3.5a, 3.5b, and 3.7b.

A consequence of nonlinear depth dependence of horizontal normal force at the rear of the thrust sheet and nonzero slopes is that the vertical stress within the block deviates from lithostatic values. Conditions causing nonlinear depth distributions of horizontal and vertical normal stresses include lateral density inhomogeneities in the lithosphere (Bott, 1990). Deviations of the vertical stresses from lithostatic values, however, must be 


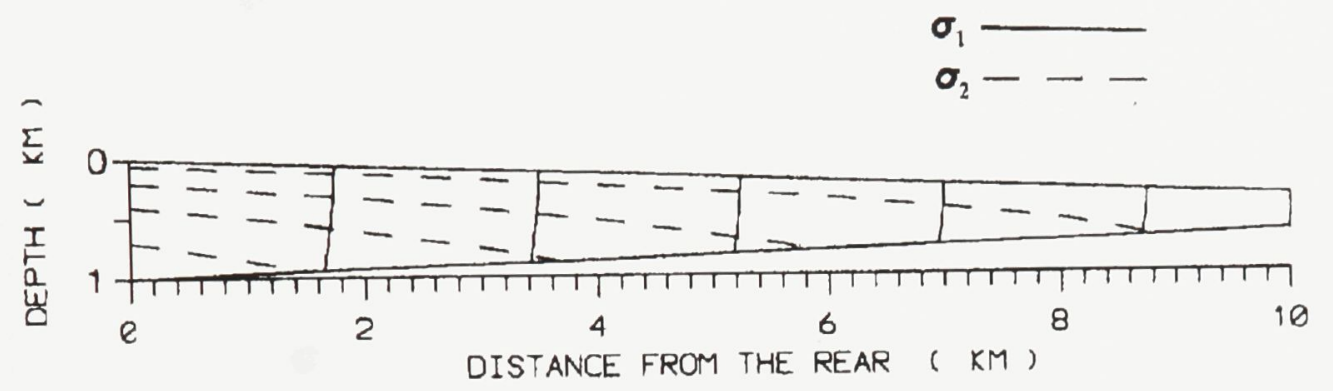

(a)

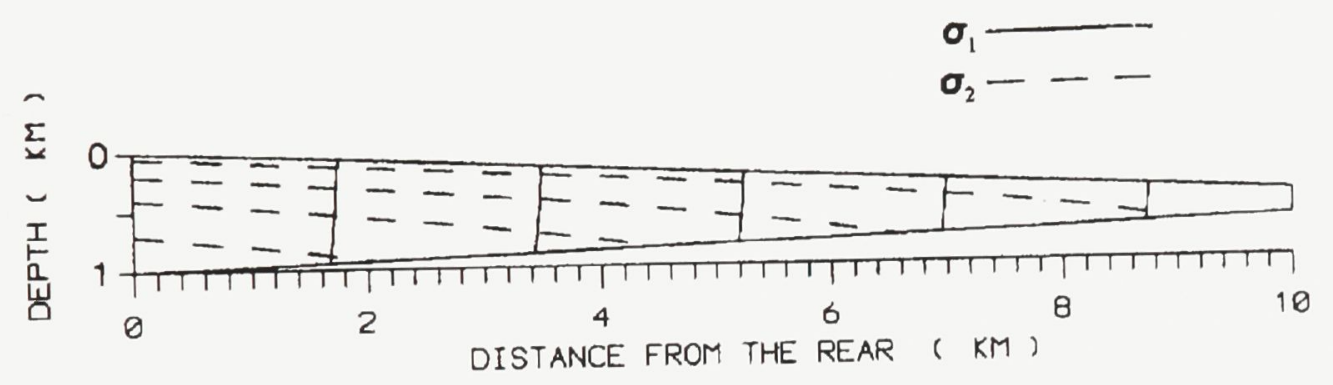

(b)

Figure 3.2 Principal stress trajectories within the wedge-shaped thrust sheet subject to nonlinear horizontal compression on the rear vertical edge for the model parameters given in Tables 3.1 and 3.2 combined: (a) case B and (b) case D. Minimum and maximum compressions are represented by the solid and the dashed lines. 

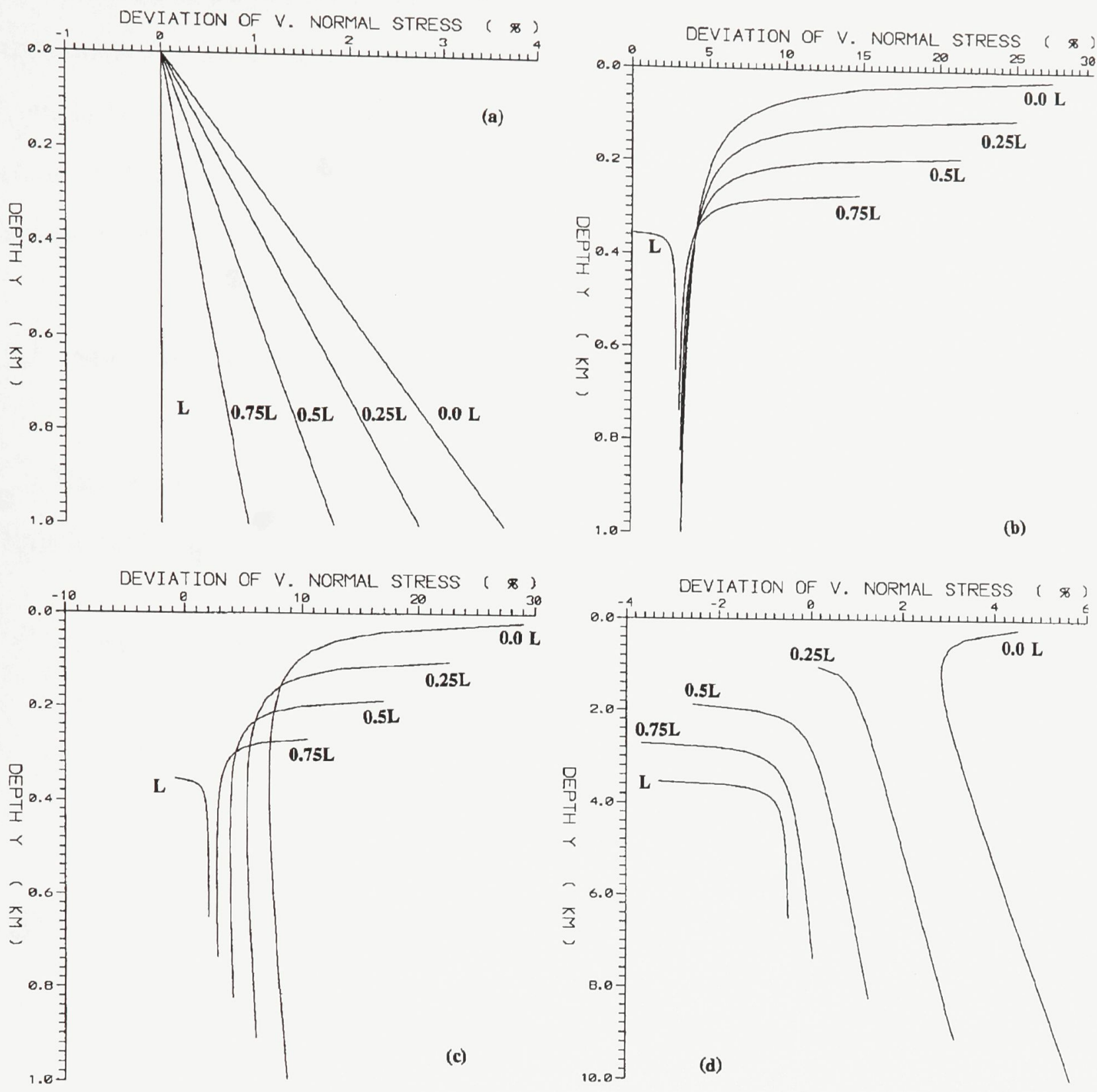

Figure 3.3 Deviation of vertical normal stress from lithostatic values for selected model parameters: (a) case B in Table 3.1, (b) case B in Table 3.2, (c) case B in Tables 3.1 and 3.2 combined, and (d) case B in Table 3.3. Numbers beside lines represent the horizontal distance between the rear edge of the block $(0.0 L)$ and the vertical profile where the deviation is calculated. 
small, since in situ stress measurements show approximately lithostatic conditions in the uppermost few kilometers of the crust (McGarr and Gay, 1978). The deviations of the vertical normal stress from the lithostatic case for several values of the parameters are shown in Figure 3.3: for both thin- and thick- skinned thrust sheets these deviations are always relatively minor $(<5-10 \%)$ and therefore are compatible with observations.

\subsection{State of stress on the basal thrust}

The normal and shear stresses on the basal surface can be derived from the last two of equations (3.8)

$$
\begin{aligned}
\sigma_{n} & =F_{y}^{B C} \cos \beta+F_{x}^{B C} \sin \beta \\
& =A_{0}+A_{1} x+A_{2} x^{2}+A_{3} x^{3} \\
\tau_{n} & =F_{x}^{B C} \cos \beta-F_{y}^{B C} \sin \beta \\
& =B_{0}+B_{1} x+B_{2} x^{2}+B_{3} x^{3}
\end{aligned}
$$

where the parameters $A_{i}$ and $B_{i}(i=0$ to 3 ) are given by

$$
\begin{aligned}
A_{0}= & -\rho g H+\frac{a}{2} H^{2}+\frac{m+n}{3}\left(2 a-a_{1}\right) H L+\left(n^{2}-m^{2}\right) b H-2(m+n) \frac{c+c_{1}}{L} H-(m+n)^{2} c \\
A_{1}= & (m+n) \rho g-\frac{a+a_{1}}{2 L} H^{2}-(m+n)^{2} \frac{2 a-a_{1}}{2} L-3(m+n) a H+3\left(m^{2}-n^{2}\right) \frac{b+b_{1}}{L} H \\
& +3 m n^{2} b+3(m+n)^{2} \frac{c+c_{1}}{L} \\
A_{2}= & 3(m+n)^{2} a+2(m+n) \frac{a+a_{1}}{L} H+2\left(2 n^{3}+3 m n^{2}-m^{3}\right) \frac{b+b_{1}}{L} \\
A_{3}= & \frac{5(m+n)^{2}}{3} \frac{a+a_{1}}{L} \\
B_{0}= & m \rho g H+\frac{2 a-a_{1}}{6} H L-\frac{b+b_{1}}{2 L} H^{2}-m b H-\frac{c+c_{1}}{L} H-(m+n) c
\end{aligned}
$$




$$
\begin{aligned}
B_{1} & =-m(m+n) \rho g-a H-(m+n) \frac{2 a-a_{1}}{3} L+\left(m^{2}-n^{2}\right) b+2 m \frac{b+b_{1}}{L} H \\
& +2(m+n) \frac{c+c_{1}}{L} \\
B_{2} & =\frac{a+a_{1}}{2 L} H+\frac{3}{2}(m+n) a+\frac{3}{2}\left(n^{2}-m^{2}\right) \frac{b+b_{1}}{L} \\
B_{3} & =-\frac{2}{3}(m+n) \frac{a+a_{1}}{L}
\end{aligned}
$$

The expressions for $A_{i}$ and $B_{i}$ have been simplified following the same procedure and assumptions as described above for the constants $k_{i}$. Also, the fact that the basal slope is small has been taken into account.

Equations $(3.13 \mathrm{a}, \mathrm{b})$ show explicitly the effects of nonlinear boundary forces and of upper and lower slopes on the state of stress along the thrust fault. These two effects are discussed separately.

\subsubsection{Nonlinear component of horizontal compression}

In order to isolate the role of nonlinear horizontal compression in determining the state of stress on the thrust fault, a simple rectangular block is considered (i.e., $n=m=$ 0 ). Then equations (3.13a), using equations (3.13b), can be written as

$$
\begin{aligned}
& \sigma_{n}=-\rho g H+\frac{a}{2} H^{2}-\frac{a+a_{1}}{2 L} H^{2} x \\
& \tau_{n}=\frac{2 a-a_{1}}{6} H L-\frac{b+b_{1}}{2 L} H^{2}-\frac{c+c_{1}}{L} H-a H x+\frac{a+a_{1}}{2 L} H x^{2}
\end{aligned}
$$

Therefore the presence of the terms $a y^{2}$ and/or $a_{1} y^{2}$ in the external driving forces has the effect of making both shear and normal stresses vary with position along the base of the thrust block. Figure 3.4 shows $\sigma_{n}$ and $\tau_{n}$ at the base of a rectangular block for the 

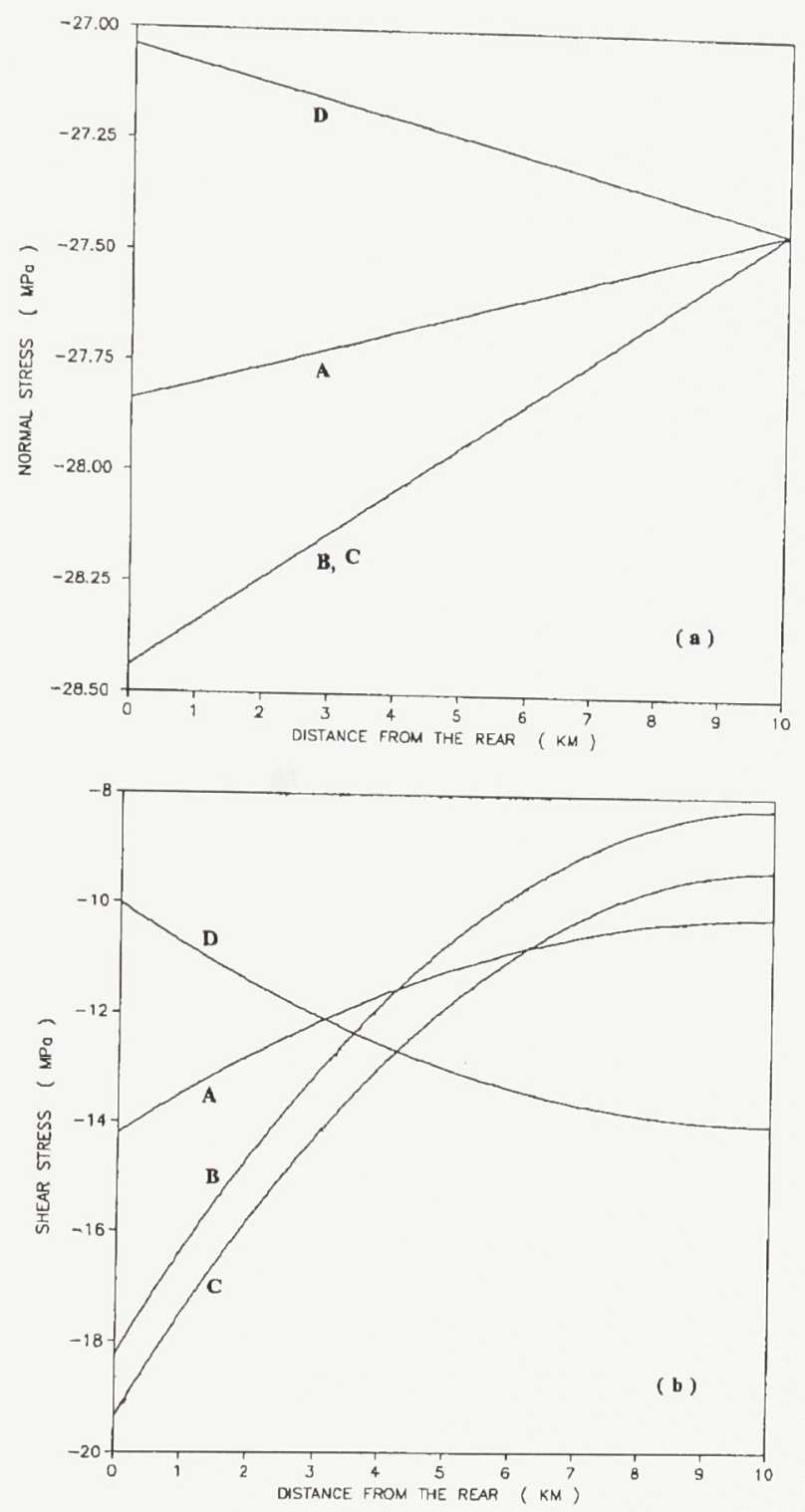

Figure 3.4 (a) Normal and (b) shear stresses along the lower boundary of a rectangular block for four different sets of force boundary conditions (given in Table 3.1). 
model parameters listed in Table 3.1, that is, nonlinear compression at the rear, and linear resistance at the front less in magnitude than the compression at the rear.

\subsubsection{Upper and lower slopes}

The upper and lower slopes of the wedge-shaped block also contribute to the variation of the state of stress on the thrust fault. In order to show this, assume $a=a_{1}=0$ in equations (3.13a); it follows that, using equations (3.13b),

$$
\begin{aligned}
\sigma_{n}= & -\rho g H+\left(m^{2}-n^{2}\right) b H-2(m+n) \frac{c+c_{1}}{L} H-(m+n)^{2} c \\
& +\left[(m+n) \rho g+3\left(m^{2}-n^{2}\right) \frac{b+b_{1}}{L} H+3 m n^{2} b+3(m+n)^{2} \frac{c+c_{1}}{L}\right] x \\
& +2\left[2 n^{3}+3 m n^{2}-m^{3}\right] \frac{b+b_{1}}{L} x^{2} \\
\tau_{n}= & m \rho g H-m b H-\frac{b+b_{1}}{2 L} H^{2}-(m+n) c-\frac{c+c_{1}}{L} H \\
& +\left[-m(m+n) \rho g+2 m \frac{b+b_{1}}{L} H+\left(m^{2}-n^{2}\right) b+2(m+n) \frac{c+c_{1}}{L}\right] x \\
& +\frac{3\left(n^{2}-m^{2}\right)}{2} \frac{b+b_{1}}{L} x^{2}
\end{aligned}
$$

It can be seen from the above equations that the effects of nonzero slopes $(n \neq 0, m \neq 0)$ on the state of stress along the wedge's basement are coupled with the force boundary conditions at the front and at the rear. In the case that the front and rear boundaries are subject to constant stresses (i.e., $a=a_{1}=b=b_{1}=0$ ), the basal normal stress $\left(\sigma_{n}\right)$ is a simple function of the wedge's taper $(m+n)$.

Some examples of the variations of normal and shear stresses on the basal thrust fault, caused by the finite slopes of the wedge, are shown in Figure 3.5 for the model parameters listed in Table 3.2. It can be seen that even for very small slopes the state of stress on the thrust fault varies significantly with position. 

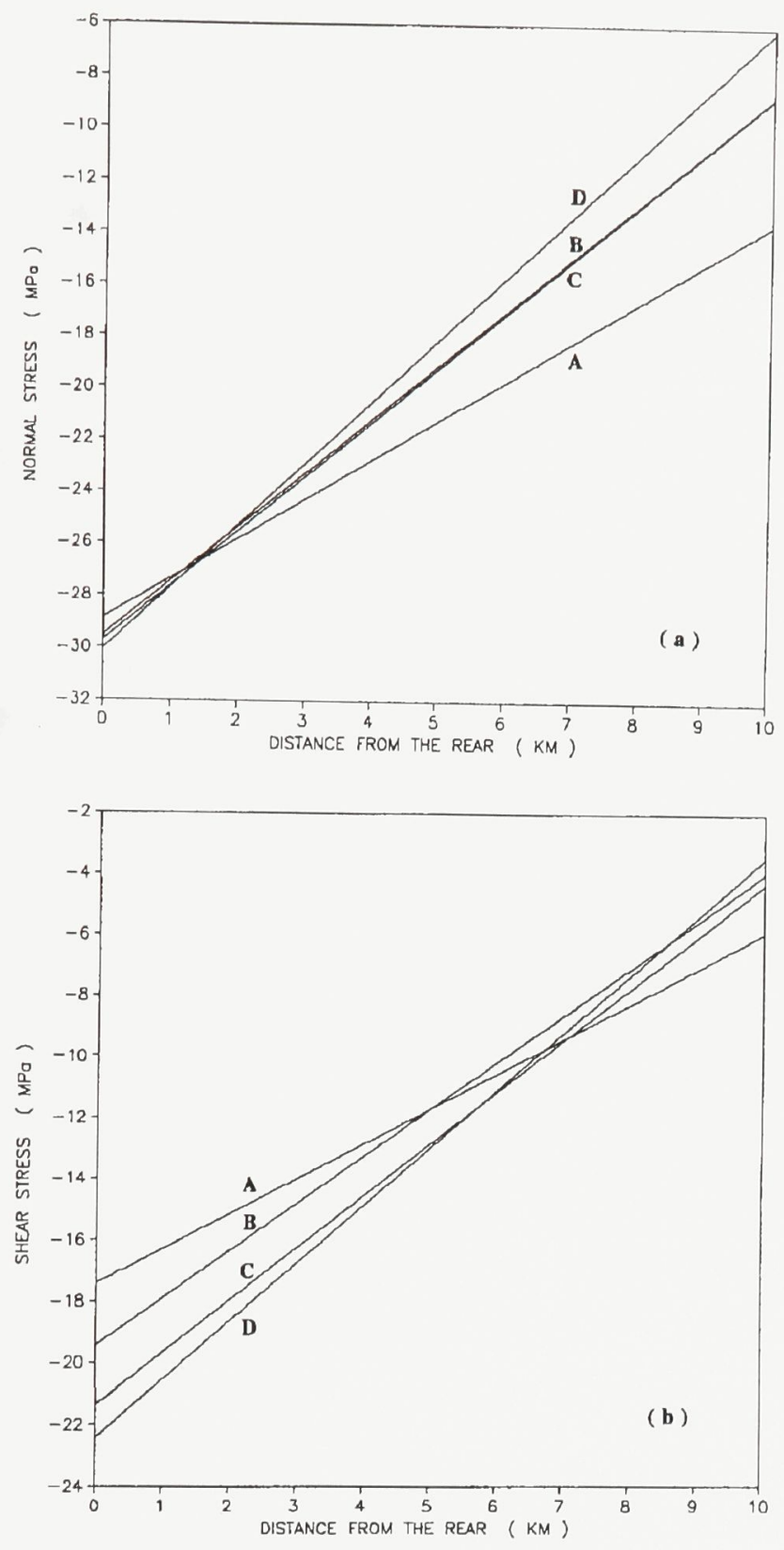

Figure 3.5 (a) Normal and (b) shear stresses along the lower boundary of a wedgeshaped block for linear boundary forces and four different sets of parameters (given in Table $3.2)$. 

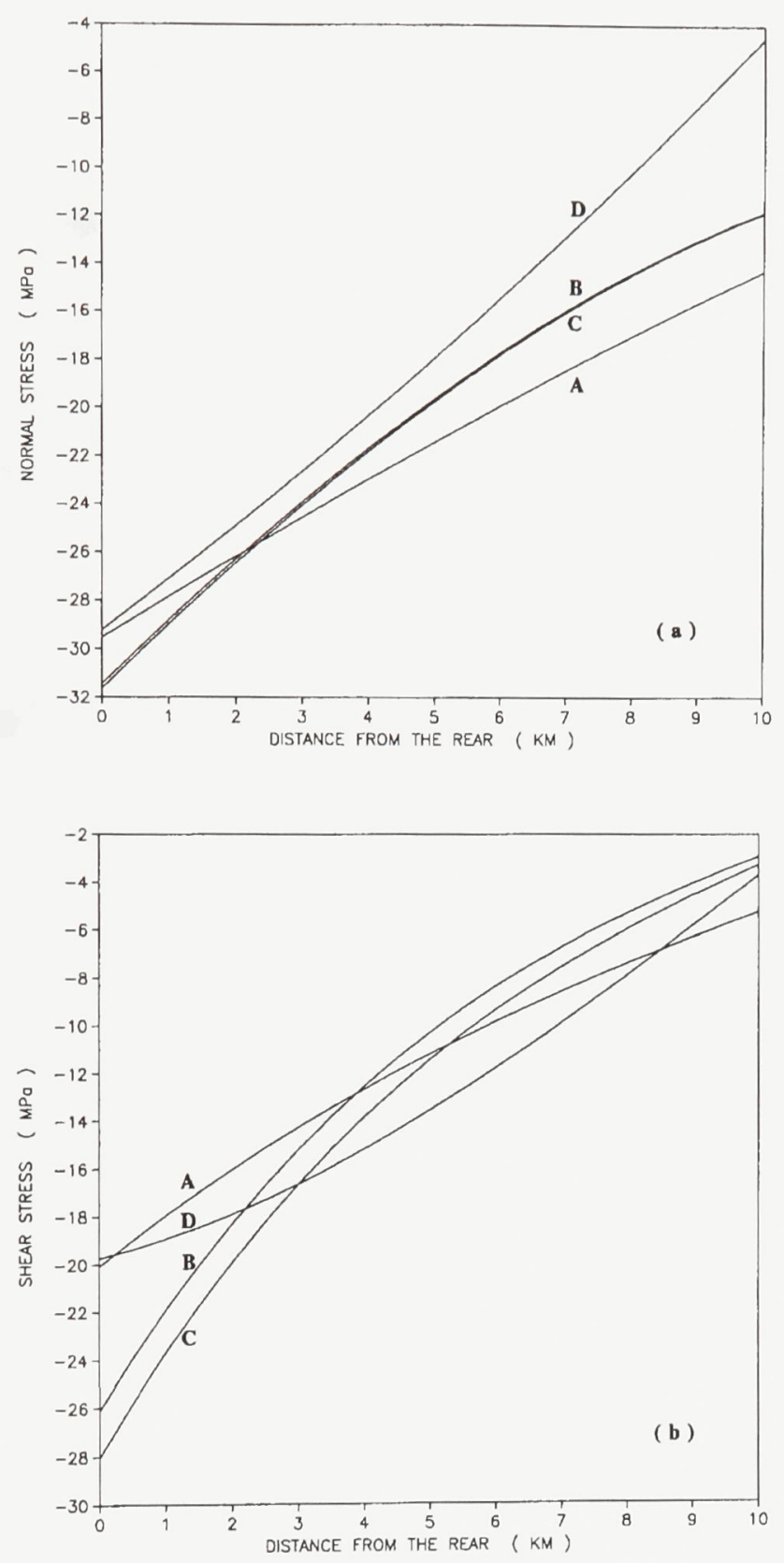

Figure 3.6 (a) Normal and (b) shear stresses along the lower boundary of a wedgeshaped block subject to nonlinear boundary forces for four different sets of parameters (given in Tables 3.1 and 3.2 combined). 
Finally, Figure 3.6 shows the combined effects of nonlinear boundary forces and of sloping upper and lower boundaries on the state of stress along the thrust fault.

\subsection{Discussion: initiation and development of thrusting}

The analytical solutions obtained in this chapter are relevant to investigate the initiation and development of slip along the basal thrust. A frictional function $\mu(x)$ along the fault can be defined as

$$
\mu(x)=\frac{\tau_{n}(x)}{\sigma_{n}(x)}
$$

If $\mu_{0}$ represents the frictional coefficient of the material, it can be assumed that the thrust fault will be activated only where $\mu(x) \geq \mu_{0}$. The inclusion of constant finite cohesion would change the value $\mu(x)$ and, consequently, decrease the length of the potentially slipped part of the fault. However, cohesion along preexisting faults is usually low (1 - 10 MPa; $c f$. Suppe, 1985; Ranalli, 1995), and therefore the frictional function defined above can be considered as a reasonable first-order approximation.

The distribution of the frictional function over the basal thrust for different boundary conditions and shapes of the block is shown in Figure 3.7. Slippage can be assumed to occur for values of the frictional function $\mu(x) \geq 0.60$ (a fair approximation for some

typical rocks such as granite and sandstone; $c f$. Jaeger and Cook, 1969). Assuming negligible cohesion, failure along the thrust, if $\mu(x)$ decreases toward the front, should begin at the rear and propagate up to the distance $x^{*}$, such that $\mu(x *) \approx 0.60$. The total length of the potentially slipped part varies with the values of the external forces and the slopes of the upper and lower boundaries. 

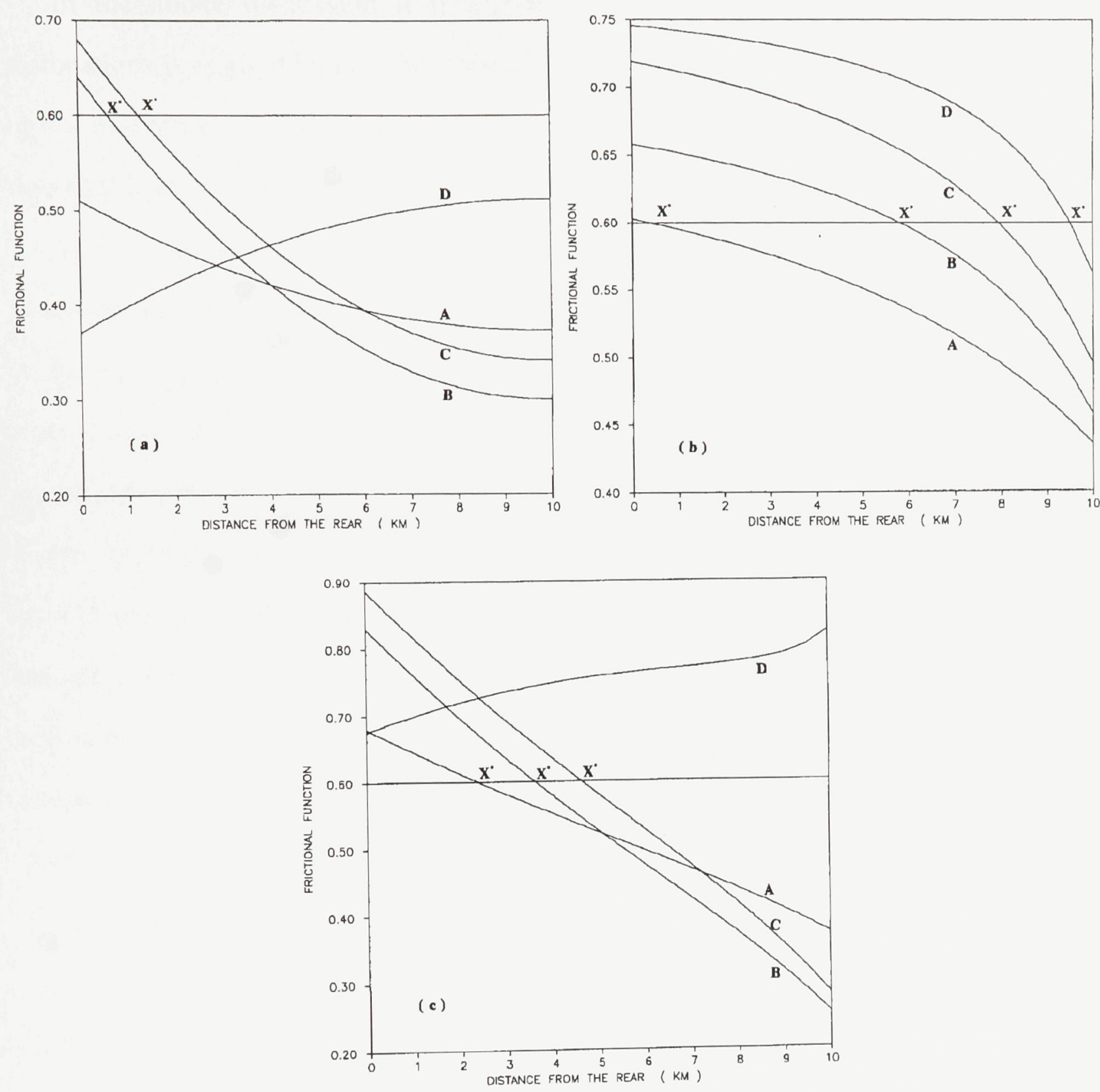

Figure 3.7 Variation of frictional function along the basal thrust for (a) rectangular block, nonlinear boundary forces, parameters as in Table 3.1; (b) wedge-shaped block, linear boundary forces, parameters as in Table 3.2; and (c) wedge-shaped block, nonlinear boundary forces, parameters as in Tables 3.1 and 3.2 combined. In each case, $x *$ denotes the distance from the rear where the frictional function equals a friction coefficient $\mu_{0}=0.60$ (represented by the horizontal line). See discussion in the text. 
In the above discussion it is implicitly assumed that the dynamic effect of dislocations is negligible, i.e., the stresses generated by a dislocation are not considered in our modelling. We are concerned mainly about the initiation and the development of thrusting from a macroscopic point of view.

Several calculations have been performed for different values of force parameters and boundary slopes. In general, combinations with $a<0$ (gradient of rear pushing force decreasing with increasing depth) result in a frictional function that decreases from the rear to the front of the thrust sheet. A nonlinear front edge force does not affect significantly this conclusion. The larger the difference between horizontal rear and front forces, the higher the frictional function and, consequently, the likelihood and extent of slip. Upper and lower slopes of the order of $2^{\circ}$ or larger, even with linear boundary forces (larger at the rear) are favourable to slip, whose likelihood decreases as the frontal resistance increases. The slip proceeds from the rear to the front, as is observed in thrust sheets. This is also the case when the gradient of the rear pushing force increases with depth.

The choice of boundary force parameters is constrained by two requirements: (1) there should be no failure within the block, and (2) the shear stress on the basal thrust should be realistic (i.e., $\tau_{n} \leq 50 \mathrm{MPa}$ ). The second requirement is satisfied in the present model. To check if the first requirement is met, the critical stress $S_{c}$ within the block according to the Coulomb-Navier criterion is computed as (Jaeger and Cook, 1969)

$$
S_{c}=\frac{1}{\cos \phi}\left[\frac{\left(\sigma_{x x}-\sigma_{y y}\right)^{2}}{4}+\sigma_{x y}^{2}\right]^{\frac{1}{2}}+\frac{\sigma_{x x}+\sigma_{y y}}{2} \tan \phi
$$


where $\phi=\tan ^{-1}\left(\mu_{0}\right)$ is the internal friction angle $\left(\approx 30^{\circ}\right.$ for $\left.\mu_{0} \approx 0.577\right)$. The critical stress maximum within the block is generally less than $50 \mathrm{MPa}$, that is, below estimated values of rock strength.

\subsection{Very large wedges and the effect of fluid pressure}

The above results are for a block with $L=10 \mathrm{~km}$ and $H=1 \mathrm{~km}$. For very large thrust blocks (e.g., $L \approx 50-100 \mathrm{~km}$ and $H \approx 5-10 \mathrm{~km}$ ) the shear stress on the basal boundary is caused in large part by the term $\left(2 a-a_{1}\right) H L / 6$ in the parameter $B_{0}$ (see equation (3.13b)), and this puts a constraint on the nonlinear component of the boundary force. Furthermore, since the maximum basal normal stress is mainly caused by the overburden pressure $(\approx \rho g H)$, and the maximum realistic value for shear stress in the crust is $\tau_{n} \approx 50-100 \mathrm{MPa}$, it is immediately clear that in the absence of pore fluid pressure on the basal thrust the maximum possible rear thickness of a cohesionless thrust sheet should not exceed $H=\tau_{n} /\left(\mu_{0} \rho g\right) \approx 6 \mathrm{~km}$, assuming density $\rho=2800 \mathrm{~kg} \mathrm{~m}^{-3}$ and friction coefficient $\mu_{0} \approx 0.60$. The presence of pore fluids along the basal thrust increases the maximum thickness of the thrust sheet and also the total slipped length because the normal stress on the fault plane is reduced to $\sigma_{n}^{*}=\sigma_{n}(1-\lambda)$, where $\lambda$ is the pore fluid factor (ratio of pressure of water column to overburden pressure). Effective normal stress, shear stress, and frictional function at the base of a 100-km-long thrust sheet are shown in Figure 3.8 for the model parameters given in Table 3.3 and a pore fluid factor $\lambda=0.75$. These parameters result in critical stresses within the block $S_{c} \leq 35 \mathrm{MPa}$ for cases A, B, and $\mathrm{C}$ and $S_{c} \leq 70 \mathrm{MPa}$ for case $\mathrm{D}$, which is considered geologically unrealistic, as it results in a frictional function increasing towards the front of the thrust sheet. The maximum deviation of vertical normal stress from lithostatic values is generally less than $6 \%$. 
Table 3.3 Values of model parameters for wedge-shaped block with dimensions $L=100$ $\mathrm{km}, H=10 \mathrm{~km}$ subject to nonlinear boundary forces and $\lambda=0.75$ on the basal thrust.

\begin{tabular}{ccccc} 
& \multicolumn{5}{c}{ Case* } \\
\cline { 2 - 5 } Parameters & $\mathrm{A}$ & $\mathrm{B}$ & $\mathrm{C}$ & $\mathrm{D}$ \\
\hline$a\left(\mathrm{MPa} / \mathrm{km}^{2}\right)$ & -0.1 & -0.2 & -0.2 & 0.1 \\
$a_{1}\left(\mathrm{MPa} / \mathrm{km}^{2}\right)$ & 0 & 0 & 0 & 0 \\
$b(\mathrm{MPa} / \mathrm{km})$ & 32.9 & 32.9 & 35.7 & 35.7 \\
$b_{1}(\mathrm{MPa} / \mathrm{km})$ & -22.0 & -22.0 & -22.0 & -22.0 \\
$c(\mathrm{MPa})$ & 110 & 110 & 110 & 120 \\
$c_{1}(\mathrm{MPa})$ & 0 & 0 & 0 & 0 \\
$\alpha$ & $2^{\circ}$ & $2^{\circ}$ & $2^{\circ}$ & $2.5^{\circ}$ \\
$\beta$ & $1^{\circ}$ & $2^{\circ}$ & $2^{\circ}$ & $2^{\circ}$ \\
\hline
\end{tabular}

* Cases A, B, C, and D refer to curves in Figures 3.8a, 3.8b, and 3.8c.

In cases where $\mu(x)$ decreases toward the front of the thrust sheet, the minimum pore fluid factor (assumed constant) required to begin slippage at the rear of the basal fault can be calculated from equation (3.16) by using the effective normal stress

$$
\begin{aligned}
\lambda & =1-\frac{\mu(x=0)}{\mu_{0}} \\
& =1-\frac{1}{\mu_{0}} \frac{B_{0}}{A_{0}}
\end{aligned}
$$

where $A_{0}$ and $B_{0}$ are constant terms of normal and shear stresses, respectively, on the basal fault (see equation (3.13b)). Figure 3.9 shows the minimum $\lambda$ as a function of the rear thickness of the wedge. It can be seen that (1) increasing the magnitude of the nonlinear part of the rear pushing force has the effect of decreasing the required minimum $\lambda$; (2) if the magnitude of $a$ is large enough (such as $a=-0.1 \mathrm{MPa} / \mathrm{km}^{2}$ in the calculation), $\lambda$ will be maximum for a thrust sheet with intermediate thickness $H$ and 

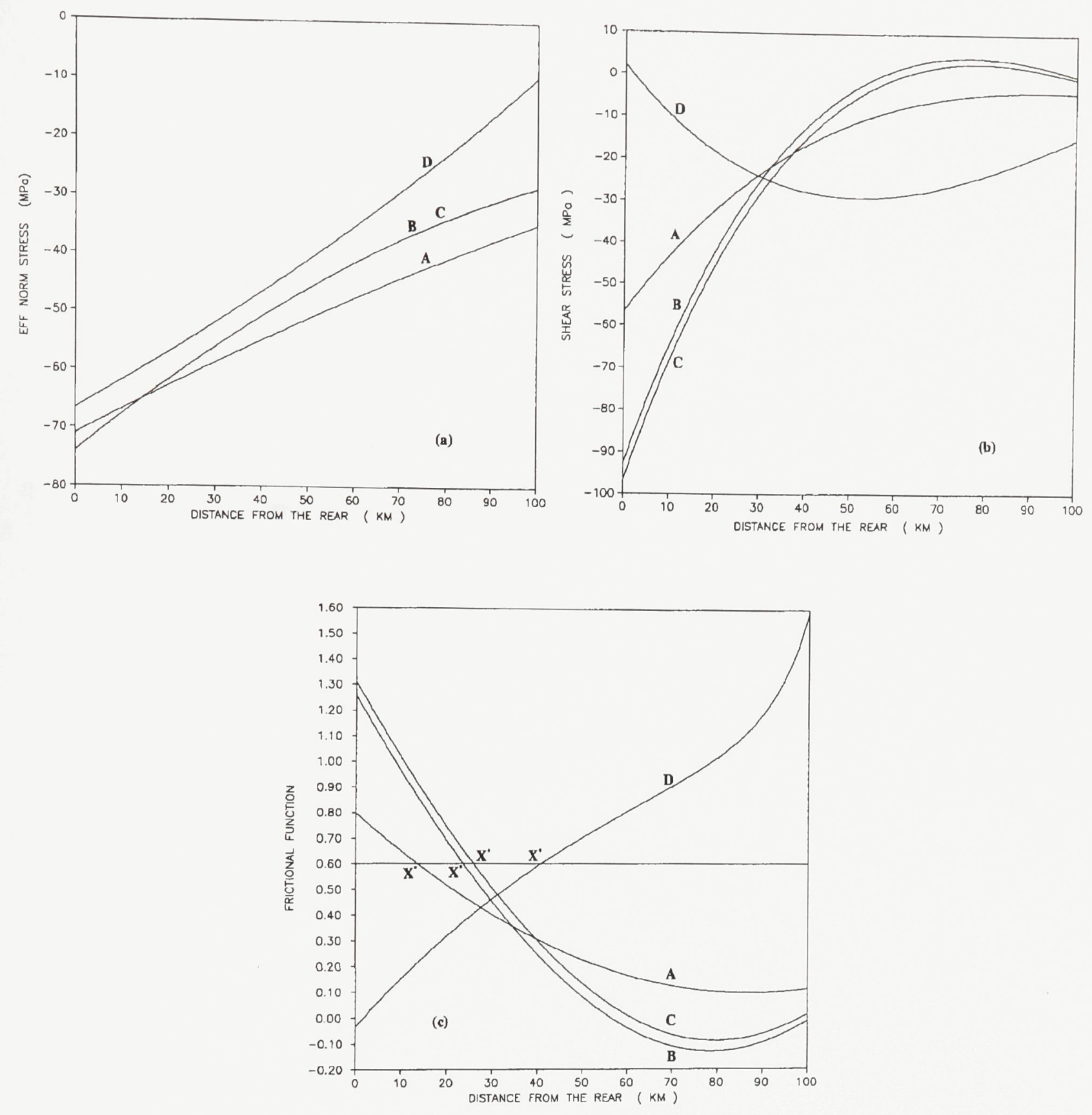

Figure 3.8 $\quad$ (a) Effective normal stress, (b) shear stress, and (c) frictional function along the basal boundary of a 100-km long wedge-shaped thrust sheet for model parameters listed in Table 3.3. A pore fluid factor $\lambda=0.75$ is assumed. 

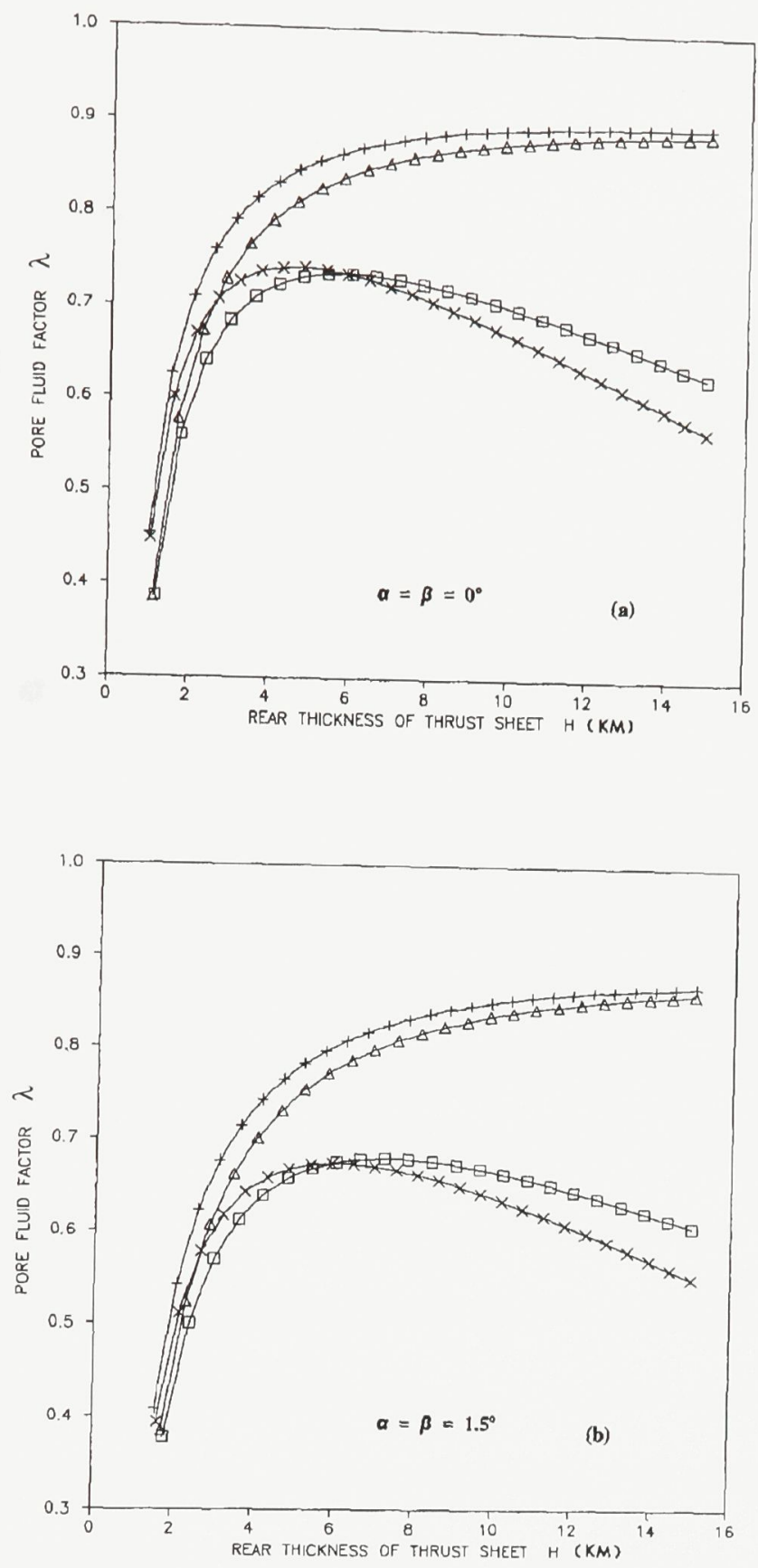

Figure 3.9 Minimum pore fluid factor $\lambda$ (as defined by equation (3.18)) as a function of rear thickness of the thrust sheet for (a) rectangular block and (b) wedge-shaped block. Parameter values, $\mu_{0}=0.60 ; a_{1}=0, b=32.9 \mathrm{MPa} / \mathrm{km}, b_{1}=-22.0 \mathrm{MPa} / \mathrm{km}, c=110 \mathrm{MPa}$, and $c_{1}=0$. In both diagrams, squares denote the case when $a=-0.1, r=0.1$; triangles, $a=$ $-0.01, r=0.1 ; \mathrm{X}, a=-0.1, r=0.08$; crosses, $a=-0.01, r=0.08$. Units of $a$ are $\mathrm{MPa} / \mathrm{km}^{2} ; r=$ $H / L$ is the aspect ratio of the thrust sheet. 
length $L$ (in the calculation, $H \approx 3-6 \mathrm{~km}$ and $L \approx 40-75 \mathrm{~km}$ ), which shows that the likelihood of initiation of slip on the basal fault in the presence of nonlinear rear pushing force does not always decrease with the increasing size of the thrust sheet (This is a consequence of the fact that, for some choices of boundary forces parameters, the shear stress on the basal fault, compared with the normal stress, will increase faster with $H$ and $L$ (for the same ratio of $r=H / L$ ) when $H>6 \mathrm{~km}$ and $L>75 \mathrm{~km}$ ); (3) the larger the upper and lower boundary slopes, the smaller the required minimum pore fluid factor; and (4) the shape of the relation between $\lambda$ and rear thickness is determined mainly by the nonlinear part of rear compression and is not affected significantly by the aspect ratio of the thrust sheet.

In general, the extent of the critical length $\left(\mu(x) \geq \mu_{0}\right)$ depends on the boundary forces and on the shape of the wedge: increasing the rear compression and/or increasing the upper and lower slopes have the effect of increasing the critical length. For a given length $L$ of the thrust sheet, increasing the rear thickness $H$ decreases the length along which slippage occurs. In all cases, only part of he fault is at stress levels compatible with failure, which supports Price's $(1988,1990)$ argument (see also Washington, 1990) that initiation of slip does not imply that the whole fault is at failure. Although simple static considerations cannot fully solve Smoluchowski's paradox, they indicate that the stress state at the base of a wedge-shaped block is compatible with slippage initiating at the rear of the block and affecting only a part of the fault at any given time. 


\section{Chapter 4}

\section{Two-Dimensional Incompressible Power-law Flow}

\subsection{Introduction}

This chapter presents a general solution for a plane strain incompressible material with steady-state power-law rheology. Stress, strain rate and velocity fields are expressed explicitly in terms of bulk rheology, force boundary conditions at the rear of a triangular wedge, and a function $C(x, y)$ which is related to the deviation of vertical normal stress from the lithostatic overburden. When the power-law stress exponent equals unity (that is, the material becomes a linear viscous Newtonian body), the function $C(x, y)$ can be determined by kinematic and/or force boundary conditions and the problem can be solved analytically.

\subsection{Model geometry and rheology}

The simplified two-dimensional geometry of an orogenic wedge and the associated coordinate system are shown in Figure 4.1. The upper boundary is a traction-free surface with a small constant slope $\alpha$ dipping toward the front. The lower boundary has a slope $\beta$ that may slightly increase from the front to the rear of the wedge. The origin of the coordinate system is fixed on the upper surface, with positive $x$ axis parallel to the surface towards the front and positive $y$ axis perpendicular to the surface and pointing downwards. 


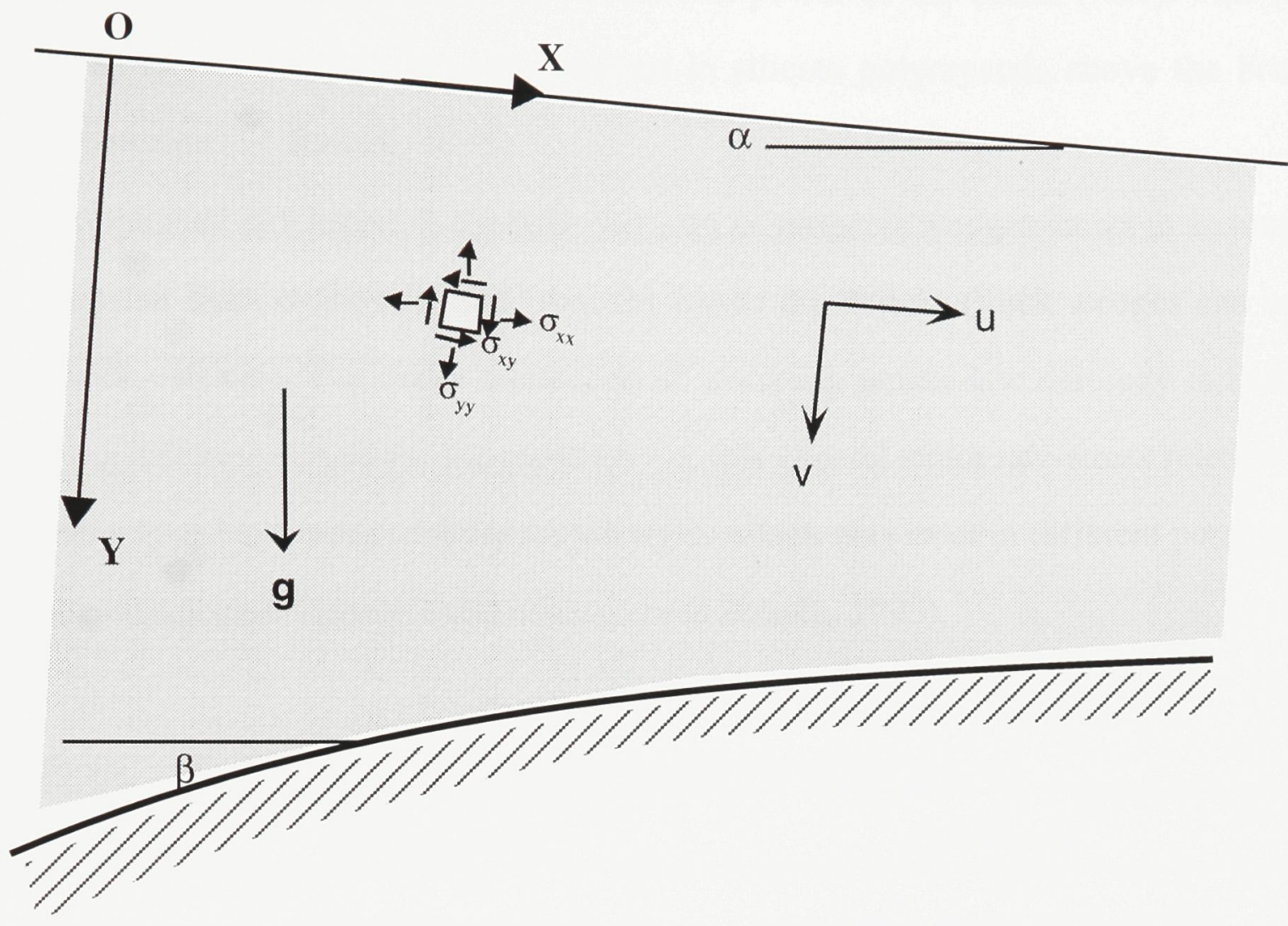

Figure 4.1 Simplified two-dimensional orogenic wedge model: $\alpha$ is the surface slope, $\beta$ the basal slope, $u, v$ the velocity components, and $g$ the acceleration of gravity. Positive stress components are shown. 
The bulk rheology of the wedge material is assumed to be steady state power-law creep, in which strain rate is related to the $n$th power of the stress $(n \geq 1)$. This nonNewtonian flow behaviour is very common in silicate polycrystals above the brittleductile transition ( $c f$. Ranalli, 1995).

As mentioned in Chapter 2, the bulk rheology of orogenic wedges varies in a complex way from the front to the rear and from the top to the bottom (thick wedges can be as deep as $30-40 \mathrm{~km}$ ). The major advantage of assuming power-law rheology is that, by specifying different values for the parameter $n$, this general strain rate-stress relation can represent a broad spectrum of deformation styles which may exist in different parts of the orogenic wedge (see Table 4.1 and discussion in Ranalli, 1995).

Table 4.1 Steady state power-law rheology and deformation patterns

\begin{tabular}{c|c|c}
\hline stress exponent $n$ & deformation style & applicable to \\
\hline$\infty$ & Perfectly plastic body & near surface $($ depth $<10-15 \mathrm{~km})$ \\
\hline $2 \sim 4$ & Power-law body & below $10-15 \mathrm{~km}$ \\
\hline 1 & Newtonian body & shear zones with sub-millimetric grain size \\
\hline
\end{tabular}

The wedge material, as a first-order approximation, is taken to be incompressible. This assumption greatly simplifies the problem, and is also supported by some geological observation ( $c f$. Dahlen and Barr, 1989 for details). However, it is not valid when there is large volume reduction due to loss of fluids.

The basic equations of power-law rheology for incompressible flow are as follows (details of derivation and discussion can be found in Ranalli, 1995):

(1) Strain rate $\dot{\varepsilon}_{i j}$ is linearly proportional to deviatoric stress $\sigma_{i j}^{\prime}$ 


$$
\dot{\varepsilon}_{i j}=\lambda \sigma_{i j}^{\prime}
$$

where the parameter $\lambda$ is a function of the stress and therefore of position;

(2) There exists a power-law dependence of effective strain rate $\dot{\varepsilon}_{E}$ on effective shear stress $\tau_{E}^{\prime}$

$$
\dot{\varepsilon}_{E}=A \tau_{E}^{\prime n}
$$

where the parameter $A$ is a function of material properties, pressure and temperature, and effective stress and strain rate are the second invariant of their respective tensors

$$
\tau_{E}^{\prime 2}=\frac{1}{2} \sigma_{i j}^{\prime} \sigma_{i j}^{\prime} \quad \dot{\varepsilon}_{E}^{2}=\frac{1}{2} \dot{\varepsilon}_{i j} \dot{\varepsilon}_{i j}
$$

Combining the above equations with equations (4.1) and (4.2), it follows that

$$
\begin{aligned}
& \dot{\varepsilon}_{E}=\lambda \tau_{E}^{\prime} \\
& \lambda=A \tau_{E}^{\prime(n-1)}
\end{aligned}
$$

So that

$$
\dot{\varepsilon}_{i j}=A \tau_{E}^{\prime(n-1)} \sigma_{i j}^{\prime}
$$

The parameter $A$ can be expressed as (see e.g. Kirby, 1983; Ranalli, 1995)

$$
A=A_{0} \exp \left(-\frac{Q+P V}{R T}\right)
$$


where $T$ is the absolute temperature, $R$ the universal gas constant, $P$ the pressure, and the parameters $Q, V$ and $A_{0}$ are material coefficients $(Q$ and $V$ are the creep activation energy and activation volume, respectively; the term $P V$ is negligible when $P<<k$ (bulk modulus) as is the case in the crust). The parameter $A_{0}$ is slightly temperature-dependent, but this is usually neglected in comparison with the exponential term.

\subsection{Basic equations}

There are three sets of equations involved in the analysis of stress, strain rate, and velocity fields in orogenic wedges (incompressibility and plane strain are assumed). They are:

(1) equations of equilibrium

$$
\begin{aligned}
& \frac{\partial \sigma_{x x}}{\partial x}+\frac{\partial \sigma_{x y}}{\partial y}+\rho g_{x}=0 \\
& \frac{\partial \sigma_{x y}}{\partial x}+\frac{\partial \sigma_{y y}}{\partial y}+\rho g_{y}=0
\end{aligned}
$$

(2) strain rate - velocity relations

$$
\begin{aligned}
& \dot{\varepsilon}_{x x}=\frac{\partial u}{\partial x} \\
& \dot{\varepsilon}_{y y}=\frac{\partial v}{\partial y}=-\frac{\partial u}{\partial x} \\
& \dot{\varepsilon}_{x y}=\frac{1}{2}\left(\frac{\partial u}{\partial y}+\frac{\partial v}{\partial x}\right)
\end{aligned}
$$

(3) constitutive equations 


$$
\begin{aligned}
& \dot{\varepsilon}_{x x}=\lambda \sigma_{x x}^{\prime} \\
& \dot{\varepsilon}_{y y}=\lambda \sigma_{y y}^{\prime} \\
& \dot{\varepsilon}_{x y}=\lambda \sigma_{x y}^{\prime}
\end{aligned}
$$

where $g_{x}$ and $g_{y}$ are the gravitational components in $x$ and $y$ directions; $\sigma_{x x}, \sigma_{y y}$, and $\sigma_{x y}$ the stress tensor components; $\rho$ the density; and $u$ and $v$ the velocity components in the $x$ and $y$ directions. The parameter $\lambda$ is defined according to equation (4.3).

The components of the gravitational acceleration are related to surface slope as

$$
g_{x}=g \sin \alpha \quad g_{y}=g \cos \alpha
$$

and the stress deviator components are

$$
\begin{aligned}
& \sigma_{x x}^{\prime}=\frac{1}{2}\left(\sigma_{x x}-\sigma_{y y}\right) \\
& \sigma_{y y}^{\prime}=\frac{1}{2}\left(\sigma_{y y}-\sigma_{x x}\right)=-\sigma_{x x}^{\prime} \\
& \sigma_{x y}^{\prime}=\sigma_{x y}
\end{aligned}
$$

\subsection{Derivation of solutions}

There are eight unknowns (i.e., three stress components, two velocity components and three strain rate components) linked by eight equations (i.e., equations (4.6) to (4.8)). Finding an analytical solution for a set of nonlinear partial differential equations has been found to be extremely difficult, and very few closed-form solutions of this problem exist (cf. Lliboutry, 1987). The approach adopted in this chapter is to express stress, strain rate, and velocity in terms of one single function (denoted by $C(x, y)$ ). If this single function can be determined or approximated, then the whole problem becomes solvable. 
The choice of $C(x, y)$ is determined by the nature of the problem. For the purpose of modelling orogenic wedges in this thesis, $C(x, y)$ is defined as the deviation of normal stress from the lithostatic overburden in the direction perpendicular to the surface of the wedge (i.e., $y$-direction in Fig. 4.1). The normal stress in the $y$-direction does not differ much from the lithostatic pressure (Elliott, 1976; Boyer and Elliott, 1982; Davis et al., 1983; Dahlen, 1984; Suppe, 1985; Platt, 1986; Liu and Ranalli, 1992, 1994; Yin, 1986, $1988,1989,1993)$ since the wedge's topographic slope is relatively small $\left(\alpha<6^{\circ}\right)$. Also, the gradient in shear stress in $x$-direction is likely to be small compared to the gradient in normal stress in the $y$-direction.

\subsubsection{Stress solution}

On the base of the above discussion, it is convenient to express the normal stress $\sigma_{y y}$ as a sum of lithostatic overburden pressure and a function $C(x, y)$

$$
\sigma_{y y}=-\bar{\rho}_{y} g_{y} y+C(x, y)
$$

where $g_{y}$ is the gravity component defined in equation (4.9) and $\bar{\rho}_{y}$ is the density averaged in the $y$-direction between the top surface $(y=0)$ and the depth $y$, so that $\bar{\rho}_{y}(x, y)=\frac{1}{y} \int_{0}^{y} \rho(x, \xi) d \xi$. The function $C(x, y)$ has the dimension of stress and represents the non-lithostatic part of normal stress in the $y$-direction.

Substituting equation (4.11) into the second of equations (4.6), one has

$$
\frac{\partial \sigma_{x y}}{\partial x}+\frac{\partial C(x, y)}{\partial y}=0
$$

from which the shear stress $\sigma_{x y}$ is readily obtained by integration 


$$
\sigma_{x y}=T(y)-\int_{0}^{x} \frac{\partial C(\tau, y)}{\partial y} d \tau
$$

where $T(y)$ is an arbitrary integration function.

To determine the normal stress component in the $x$-direction $\left(\sigma_{x x}\right)$, we combine equation (4.13) with the first of equations (4.6)

$$
\frac{\partial \sigma_{x x}}{\partial x}=-T^{\prime}(y)-\rho g_{x}+\int_{0}^{x} \frac{\partial^{2} C(\tau, y)}{\partial y^{2}} d \tau
$$

Integration equation (4.14) with respect to $x$ gives

$$
\sigma_{x x}=S(y)-x T^{\prime}(y)-\bar{\rho}_{x} g_{x} x+\int_{0}^{x} \int_{0}^{\varsigma} \frac{\partial^{2} C(\tau, y)}{\partial y^{2}} d \tau d \varsigma
$$

where $S(y)$ is another arbitrary integration function and $\bar{\rho}_{x}$ is the density averaged in the $x$-direction between 0 and $x$, that is, $\bar{\rho}_{x}(x, y)=\frac{1}{x} \int_{0}^{x} \rho(\tau, y) d \tau$.

Combination of equations (4.11), (4.13) and (4.15) provides a general solution to the equilibrium equations

$$
\begin{aligned}
& \sigma_{x x}=S(y)-x T^{\prime}(y)-\bar{\rho}_{x} g_{x} x+\int_{0}^{x} \int_{0}^{\varsigma} \frac{\partial^{2} C(\tau, y)}{\partial y^{2}} d \tau d \varsigma \\
& \sigma_{y y}=-\bar{\rho}_{y} g_{y} y+C(x, y) \\
& \sigma_{x y}=T(y)-\int_{0}^{x} \frac{\partial C(\tau, y)}{\partial y} d \tau
\end{aligned}
$$


The solution is greatly simplified if the function $C(x, y)$ can be neglected when compared with the lithostatic overburden. In this case, equations (4.16) become

$$
\begin{aligned}
\sigma_{x x} & =S(y)-x T^{\prime}(y)-\bar{\rho}_{x} g_{x} x \\
\sigma_{y y} & =-\bar{\rho}_{y} g_{y} y \\
\sigma_{x y} & =T(y)
\end{aligned}
$$

(which could be further simplified if $\alpha \approx 0^{\circ}, g_{x} \approx 0$ ).

The normal stress in the $x$-direction and the shear stress on the rear "backstop" of the wedge $(x=0)$ can be easily found from equations (4.16)

$$
\begin{aligned}
& \sigma_{x x}(0, y)=S(y) \\
& \sigma_{x y}(0, y)=T(y)
\end{aligned}
$$

Equations (4.18) show that the functions $S(y)$ and $T(y)$ have a precise physical meaning.

Additional constraints on the functions $T(y)$ and $C(x, y)$ are imposed from the condition that the upper boundary of the wedge is traction-free $\left(\sigma_{y y}=\sigma_{x y}=0\right)$, i.e.,

$$
\begin{aligned}
T(0) & =0 \\
C(x, 0) & =0 \\
{[\partial C / \partial y]_{y=0} } & =0
\end{aligned}
$$

Equations (4.16) - or, in simplified form, equations (4.17) when $C(x, y)=0$ - give the general solution for stress in the wedge, in terms of functions $S(y), T(y)$, and $C(x, y)$, each of which with a precise physical meaning. Their role will be discussed further in the remainder of this chapter. 


\subsubsection{Strain rate solution}

Strain rate components are related to the corresponding deviatoric stress components by equations (4.8) which, combined with equations (4.10) and (4.16) lead to the following expressions

$$
\begin{aligned}
& \dot{\varepsilon}_{x x}=\lambda \sigma_{x x}^{\prime}=\frac{\lambda}{2}\left[S(y)-x T^{\prime}(y)+\bar{\rho}_{y} g_{y} y-\bar{\rho}_{x} g_{x} x+\int_{0}^{x} \int_{0}^{\zeta} \frac{\partial^{2} C(\tau, y)}{\partial y^{2}} d \tau d \varsigma-C(x, y)\right] \\
& \dot{\varepsilon}_{y y}=\lambda \sigma_{y y}^{\prime}=-\dot{\varepsilon}_{x x} \\
& \dot{\varepsilon}_{x y}=\lambda \sigma_{x y}^{\prime}=\lambda\left[T(y)-\int_{0}^{x} \frac{\partial C(\tau, y)}{\partial y} d \tau\right]
\end{aligned}
$$

The parameter $\lambda$ can be expressed in terms of either the second invariant of stress $\left(\tau_{E}^{\prime}\right)$ or the second invariant of strain rate $\left(\dot{\varepsilon}_{E}\right)$ as (see equations (4.2) and (4.3))

$$
\lambda=A \tau_{E}^{\prime(n-1)}=A^{\frac{1}{n}} \dot{\varepsilon}_{E}^{\frac{n-1}{n}}
$$

where

$$
\begin{aligned}
& \tau_{E}^{\prime}=\left({\sigma_{x x}^{\prime}}^{2}+{\sigma_{x y}^{\prime}}^{2}\right)^{\frac{1}{2}}=\left[\frac{1}{4}\left(\sigma_{x x}-\sigma_{y y}\right)^{2}+\sigma_{x y}{ }^{2}\right]^{\frac{1}{2}} \\
& \dot{\varepsilon}_{E}=\left(\dot{\varepsilon}_{x x}{ }^{2}+\dot{\varepsilon}_{x y}{ }^{2}\right)^{\frac{1}{2}}
\end{aligned}
$$

Again, the expression for $\lambda$ is rather complicated (see equations (4.16) and (4.20)), but it is simplified if $C(x, y) \approx 0$. Even in this latter case, however, it is a nonlinear function of position unless functions $S$ and $T$ and material parameters (i.e., $A$ and $n$ ) are constant. 


\subsubsection{Velocity solution}

The velocity components can also be expressed as functions of the stress components. From the first two of equations (4.7), one obtains

$$
\begin{aligned}
& u(x, y)=\int_{0}^{x} \dot{\varepsilon}_{x x}(\xi, y) d \xi+H(y) \\
& v(x, y)=-\int_{0}^{y} \dot{\varepsilon}_{x x}(x, \tau) d \tau+I(x)
\end{aligned}
$$

where $I(x)$ and $H(y)$ are two integration functions. Physically, $H(y)$ is the vertical distribution of the longitudinal velocity component at the backstop since $H(y)=$ $u(x=0, y)$; and $I(x)$ is the longitudinal distribution of vertical velocity component on the top surface since $I(x)=v(x, y=0)$

Substituting equations (4.22) into the third of equations (4.7) gives

$$
\int_{0}^{x} \frac{\partial \dot{\varepsilon}_{x x}(\tau, y)}{\partial y} d \tau-\int_{0}^{y} \frac{\partial \dot{\varepsilon}_{x x}(x, \xi)}{\partial x} d \xi-2 \dot{\varepsilon}_{x y}(x, y)+H^{\prime}(y)+I^{\prime}(x)=0
$$

This is the compatibility equation which the strain rate and velocity fields have to satisfy. Note that also the function $C(x, y)$ is required to satisfy this equation, because strain rate components are functions of the stress solution (see equations (4.16)). Combination of equations (4.20), (4.21) and (4.23) gives

$$
\begin{array}{r}
\int_{0}^{x} \frac{\partial}{\partial y}\left[A\left(\sigma_{x x}^{\prime 2}+\sigma_{x y}^{\prime 2}\right)^{\frac{n-1}{2}} \sigma_{x x}^{\prime}\right] d x-\int_{0}^{y} \frac{\partial}{\partial x}\left[A\left(\sigma_{x x}^{\prime 2}+\sigma_{x y}^{\prime 2}\right)^{\frac{n-1}{2}} \sigma_{x x}^{\prime}\right] d y \\
-2 A\left(\sigma_{x x}^{\prime 2}+\sigma_{x y}^{\prime 2}\right)^{\frac{n-1}{2}} \sigma_{x y}^{\prime}+H^{\prime}(y)+I^{\prime}(x)=0
\end{array}
$$


where

$$
\begin{aligned}
& \sigma_{x x}^{\prime}=\frac{1}{2}\left[S(y)-x T^{\prime}(y)+\int_{0}^{x} \int_{0}^{\varsigma} \frac{\partial^{2} C(\tau, y)}{\partial y^{2}} d \tau d \varsigma-\bar{\rho}_{x} g_{x} x+\bar{\rho}_{y} g_{y} y-C(x, y)\right] \\
& \sigma_{x y}^{\prime}=T(y)-\int_{0}^{x} \frac{\partial C(\tau, y)}{\partial y} d \tau
\end{aligned}
$$

Although the set of equations (4.22 - 4.24) formally give a velocity solution, this solution (as in the case of strain rate) cannot be given explicitly unless the unknown functions are determined. This problem is considered in the next section.

\subsection{Determination of functions $I(x)$ and $H(y)$}

There are in total five unknown functions involved in the expressions for stress, strain rate and velocity fields ( $c f$. equations (4.16), (4.20) and (4.22)): $S(y), T(y), H(y), I(x)$, and $C(x, y)$. Function $I(x)$ cannot be specified as part of the boundary conditions on the wedge's top surface since a stress boundary condition (i.e., traction-free) has been assumed on the same surface (see section 4.4.1). Functions $S(y)$ and $H(y)$ represent the normal stress and $x$-direction velocity component along wedge's rear boundary, respectively, and therefore cannot be specified independently from each other. In this section, therefore, the functions $S(y)$ and $T(y)$ are taken as assigned stress boundary conditions along the wedge's rear boundary, and consequently $H(y), I(x)$, and $C(x, y)$ are the three functions to be determined from the other boundary conditions. First, it will be shown that $H(y)$ and $I(x)$ can be expressed as functions of the material bulk rheology (e.g. parameter $A$ and stress exponent $n)$ and the function $C(x, y)$. To simplify the derivation, define a function $R$ such as ( $c f$. equation (4.24a)) 


$$
\begin{aligned}
R(x, y)= & \int_{0}^{x} \frac{\partial}{\partial y}\left[A\left(\sigma_{x x}^{\prime 2}+\sigma_{x y}^{\prime 2}\right)^{\frac{n-1}{2}} \sigma_{x x}^{\prime}\right] d x-\int_{0}^{y} \frac{\partial}{\partial x}\left[A\left(\sigma_{x x}^{\prime 2}+\sigma_{x y}^{\prime 2}\right)^{\frac{n-1}{2}} \sigma_{x x}^{\prime}\right] d y \\
& -2 A\left(\sigma_{x x}^{\prime 2}+\sigma_{x y}^{\prime 2}\right)^{\frac{n-1}{2}} \sigma_{x y}^{\prime}
\end{aligned}
$$

where the deviatoric components of stress $\left(\sigma_{x x}^{\prime}, \sigma_{x y}^{\prime}\right)$ are given by equation (4.24b). Obviously, the function $R(x, y)$ can be determined once the function $C(x, y)$ is known. Also, $R(x, y)$ is independent of both $I(x)$ and $H(y)$.

Now equation (4.24a) can be rewritten as

$$
R(x, y)+H^{\prime}(y)+I^{\prime}(x)=0
$$

From which it follows

$$
\begin{array}{r}
R\left(x_{0}, y_{0}\right)+H^{\prime}\left(y_{0}\right)+I^{\prime}\left(x_{0}\right)=0 \\
R\left(x, y_{0}\right)+H^{\prime}\left(y_{0}\right)+I^{\prime}(x)=0 \\
R\left(x_{0}, y\right)+H^{\prime}(y)+I^{\prime}\left(x_{0}\right)=0
\end{array}
$$

where $\left(x_{0}, y_{0}\right)$ are the coordinates of a point within the wedge where the velocity components $u_{0}=u\left(x_{0}, y_{0}\right), v_{0}=v\left(x_{0}, y_{0}\right)$ are specified as the kinematic condition necessary to determine the velocity field.

Equations (4.27) imply that

$$
\begin{aligned}
I^{\prime}(x) & =I^{\prime}\left(x_{0}\right)+R\left(x_{0}, y_{0}\right)-R\left(x, y_{0}\right) \\
H^{\prime}(y) & =-I^{\prime}\left(x_{0}\right)-R\left(x_{0}, y\right)
\end{aligned}
$$


Integrating the first and the second of equations (4.28) with respect to $x$ and $y$, respectively, we have

$$
\begin{aligned}
& I(x)=I\left(x_{0}\right)+\left[I^{\prime}\left(x_{0}\right)+R\left(x_{0}, y_{0}\right)\right]\left(x-x_{0}\right)-\int_{x_{0}}^{x} R\left(\xi, y_{0}\right) d \xi \\
& H(y)=H\left(y_{0}\right)-I^{\prime}\left(x_{0}\right)\left(y-y_{0}\right)-\int_{y_{0}}^{y} R\left(x_{0}, \tau\right) d \tau
\end{aligned}
$$

Obviously, the functions $I(x)$ and $H(y)$ are determined once three constants $I\left(x_{0}\right)$, $H\left(y_{0}\right)$ and $I^{\prime}\left(x_{0}\right)$ become known. The values of $I\left(x_{0}\right)$ and $H\left(y_{0}\right)$ can be determined as a function of $C(x, y)$ (through the longitudinal strain rate $\left.\dot{\varepsilon}_{x x}\right)$ by equation (4.22) since

$$
\begin{aligned}
& u_{0}=u\left(x_{0}, y_{0}\right)=\int_{0}^{x_{0}} \dot{\varepsilon}_{x x}\left(\xi, y_{0}\right) d \xi+H\left(y_{0}\right) \\
& v_{0}=v\left(x_{0}, y_{0}\right)=-\int_{0}^{y_{0}} \dot{\varepsilon}_{x x}\left(x_{0}, \tau\right) d \tau+I\left(x_{0}\right)
\end{aligned}
$$

where the longitudinal strain rate $\dot{\varepsilon}_{x x}(x, y)$ is given by equations (4.20) and (4.21). It follows from equation (4.30a) that

$$
\begin{aligned}
& H\left(y_{0}\right)=u_{0}-\int_{0}^{x_{0}} \dot{\varepsilon}_{x x}\left(\xi, y_{0}\right) d \xi \\
& I\left(x_{0}\right)=v_{0}+\int_{0}^{y_{0}} \dot{\varepsilon}_{x x}\left(x_{0}, \tau\right) d \tau
\end{aligned}
$$

Therefore, from equations (4. 22), (4. 29), and (4.30b) the velocity expression now can be written as 


$$
\begin{aligned}
& u(x, y)=u_{0}-I^{\prime}\left(x_{0}\right)\left(y-y_{0}\right)-\int_{0}^{x_{0}} \dot{\varepsilon}_{x x}\left(\xi, y_{0}\right) d \xi+\int_{0}^{x} \dot{\varepsilon}_{x x}(\xi, y) d \xi-\int_{y_{0}}^{y} R\left(x_{0}, \tau\right) d \tau \\
& v(x, y)=v_{0}+\left[I^{\prime}\left(x_{0}\right)+R\left(x_{0}, y_{0}\right)\right]\left(x-x_{0}\right)+\int_{0}^{y_{0}} \dot{\varepsilon}_{x x}\left(x_{0}, \tau\right) d \tau-\int_{0}^{y} \dot{\varepsilon}_{x x}(x, \tau) d \tau-\int_{x_{0}}^{x} R\left(\xi, y_{0}\right) d \xi
\end{aligned}
$$

Now the only unknown term in the velocity expression, beside the function $C(x, y)$, is $I^{\prime}\left(x_{0}\right)$, which can be determined in several ways. One such way is to specify the velocity $\left(u_{1}, v_{1}\right)$ at another point $\left(x_{1}, y_{1}\right)$ within the block, that is, $u_{1}=u\left(x_{1}, y_{1}\right)$ and $v_{1}=v\left(x_{1}, y_{1}\right)$. Thus, applying equations (4.31) gives

$$
\begin{aligned}
& u_{1}=u_{0}-I^{\prime}\left(x_{0}\right)\left(y_{1}-y_{0}\right)-\int_{0}^{x_{0}} \dot{\varepsilon}_{x x}\left(\xi, y_{0}\right) d \xi+\int_{0}^{x_{1}} \dot{\varepsilon}_{x x}\left(\xi, y_{1}\right) d \xi-\int_{y_{0}}^{y_{1}} R\left(x_{0}, \tau\right) d \tau \\
& v_{1}=v_{0}+\left[I^{\prime}\left(x_{0}\right)+R\left(x_{0}, y_{0}\right)\right]\left(x_{1}-x_{0}\right)+\int_{0}^{y_{0}} \dot{\varepsilon}_{x x}\left(x_{0}, \tau\right) d \tau-\int_{0}^{y_{1}} \dot{\varepsilon}_{x x}\left(x_{1}, \tau\right) d \tau-\int_{x_{0}}^{x_{1}} R\left(\xi, y_{0}\right) d \xi
\end{aligned}
$$

which leads to

$$
I^{\prime}\left(x_{0}\right)=-\frac{1}{y_{1}-y_{0}}\left[u_{1}-u_{0}+\int_{0}^{x_{0}} \dot{\varepsilon}_{x x}\left(\xi, y_{0}\right) d \xi-\int_{0}^{x_{1}} \dot{\varepsilon}_{x x}\left(\xi, y_{1}\right) d \xi+\int_{y_{0}}^{y_{1}} R\left(x_{0}, \tau\right) d \tau\right]
$$

and

$$
\begin{aligned}
R\left(x_{0}, y_{0}\right)= & \frac{1}{x_{1}-x_{0}}\left[v_{1}-v_{0}-\int_{0}^{y_{0}} \dot{\varepsilon}_{x x}\left(x_{0}, \tau\right) d \tau+\int_{0}^{y_{1}} \dot{\varepsilon}_{x x}\left(x_{1}, \tau\right) d \tau+\int_{x_{0}}^{x_{1}} R\left(\xi, y_{0}\right) d \xi\right] \\
& +\frac{1}{y_{1}-y_{0}}\left[u_{1}-u_{0}+\int_{0}^{x_{0}} \dot{\varepsilon}_{x x}\left(\xi, y_{0}\right) d \xi-\int_{0}^{x_{1}} \dot{\varepsilon}_{x x}\left(\xi, y_{1}\right) d \xi+\int_{y_{0}}^{y_{1}} R\left(x_{0}, \tau\right) d \tau\right]
\end{aligned}
$$


Note that, since the term $I^{\prime}\left(x_{0}\right)$ is found in both equations (4.32), the two velocity components $u_{1}, v_{1}$ cannot be specified independently of $C(x, y)$ (and therefore $R(x, y)$ ). The relation between $u_{1}, v_{1}$ and $R(x, y)$ is represented by equation (4.33b). Conversely, if both $u_{1}$ and $v_{1}$ are specified independently from each other, equation (4.33b) is the additional constraint on the determination of function $C(x, y)$.

In the expression for the velocity field, it is always assumed that equation (4.33b) is satisfied by the function $R(x, y)$ and by the velocity specification at point $\left(x_{1}, y_{1}\right)$. Consequently, from equations (4.31) and (4.33a) the velocity solution can be expressed as

$$
\begin{aligned}
u(x, y)= & u_{0}+\frac{y-y_{0}}{y_{1}-y_{0}}\left[u_{1}-u_{0}+\int_{0}^{x_{0}} \dot{\varepsilon}_{x x}\left(\xi, y_{0}\right) d \xi-\int_{0}^{x_{1}} \dot{\varepsilon}_{x x}\left(\xi, y_{1}\right) d \xi+\int_{y_{0}}^{y_{1}} R\left(x_{0}, \tau\right) d \tau\right] \\
& -\int_{0}^{x_{0}} \dot{\varepsilon}_{x x}\left(\xi, y_{0}\right) d \xi+\int_{0}^{x} \dot{\varepsilon}_{x x}(\xi, y) d \xi-\int_{y_{0}}^{y} R\left(x_{0}, \tau\right) d \tau \\
v(x, y)= & v_{0}+\frac{x-x_{0}}{x_{1}-x_{0}}\left[v_{1}-v_{0}-\int_{0}^{y_{0}} \dot{\varepsilon}_{x x}\left(x_{0}, \tau\right) d \tau+\int_{0}^{y_{1}} \dot{\varepsilon}_{x x}\left(x_{1}, \tau\right) d \tau+\int_{x_{0}}^{x_{1}} R\left(\xi, y_{0}\right) d \xi\right] \\
& +\int_{0}^{y_{0}} \dot{\varepsilon}_{x x}\left(x_{0}, \tau\right) d \tau-\int_{0}^{y} \dot{\varepsilon}_{x x}(x, \tau) d \tau-\int_{x_{0}}^{x} R\left(\xi, y_{0}\right) d \xi
\end{aligned}
$$

Equations (4.34) give a general solution for the velocity field in terms of $C(x, y)$ and the bulk rheology of the wedge (i.e., parameters $A$ and $n$ as in equation (4.3)), since both the function $R(x, y)$ and the longitudinal strain rate $\dot{\varepsilon}_{x x}(x, y)$ are functions of $C(x, y)$ and $A$ and $n$ (see equations (4.20) and (4.25)). As discussed in section 4.4, once the function $C(x, y)$ is determined by some means (e.g., boundary condition along the wedge base), equations (4.16), (4.20), and (4.34) represent a complete closed-form solution for a plane strain incompressible material with steady-state power-law rheology. In the next section it is shown how the function $C(x, y)$ can be determined by force and/or velocity boundary conditions in the case of Newtonian rheology. 


\subsection{The function $C(x, y)$ in the case of Newtonian rheology}

It has been proven in the previous sections that a complete analytical solution for twodimensional power-law flow in an orogenic wedge can be explicitly expressed in terms of the function $C(x, y)$, which is constrained by the compatibility requirements expressed by equations (4.24) and/or by the kinematic requirements expressed by equation (4.33b). However, the derivation of an analytical expression for $C(x, y)$ from equations (4.24) is extremely difficult. Numerical approaches have to be applied at this stage in order to make the problem solvable. Analytical solutions, however, are obtainable for some special cases (e.g., power-law rheology with $C(x, y)=0$, which is considered in the next chapter). In this section, a Newtonian rheology is considered. A general power-law flow with linear longitudinal strain rate will be considered in the next chapter.

If the wedge has linear Newtonian rheology, i.e., stress exponent $n=1$, the parameter $\lambda$ becomes independent of the state of stress (see, equation (4.3)), although it can still be a function of position (because of changes in temperature and/or material). Formally, $\lambda$ is related to the inverse of the viscosity of a Newtonian material $(\eta)$.

Assume that the function $C(x, y)$ can be expressed in polynomial form, i.e., by the following two-dimensional series

$$
C(x, y)=\sum_{i=1}^{m} \sum_{j=1}^{n} k_{i j} x^{i-1} y^{j-1}
$$

where $m$ and $n$ are positive integers and $k_{i j}(i=1$ to $m, j=1$ to $n)$ are arbitrary constants and are to be determined by equations (4.24) with $n=1$ and appropriate boundary conditions. The following derivation will show that compatibility requirements and boundary conditions can be expressed as linear systems of equations in terms of the 
coefficients $k_{i j}$. This means that the function $C(x, y)$ becomes determinable by numerical procedures.

\section{Compatibility Requirements}

The compatibility conditions (equations (4.23) and (4.24)) can be expressed in another form by differentiating equation (4.24a) with respect to $x$ and $y$, which gives

$$
\frac{\partial^{2} R}{\partial x \partial y}=\frac{\partial^{2} \dot{\varepsilon}_{x x}}{\partial y^{2}}-\frac{\partial^{2} \dot{\varepsilon}_{x x}}{\partial x^{2}}-2 \frac{\partial^{2} \dot{\varepsilon}_{x y}}{\partial x \partial y}=0
$$

where $R$ is given by equation (4.25) and the strain rate components are determined by equation (4.20). The above is the usual form of the two-dimensional compatibility relation in terms of strain rate components (see e.g. Ranalli, 1995).

In order to transform equation (4.36) into a linear system of equations in terms of $k_{i j}$, the strain rate components are expressed in terms of $k_{i j}$ first. Combination of equations (4.35) and (4.20) leads to

$$
\begin{aligned}
\dot{\varepsilon}_{x x} & =\frac{S(y)-x T^{\prime}(y)+\bar{\rho}_{y} g_{y} y-\bar{\rho}_{x} g_{x} x+\sum_{i=1}^{m} \sum_{j=1}^{n}\left[\frac{(j-1)(j-2)}{i(i+1)} x^{2}-y^{2}\right] x^{i-1} y^{j-3} k_{i j}}{4 \eta(x, y)} \\
& =X(x, y)+\sum_{i=1}^{m} \sum_{j=1}^{n} a_{i j}(x, y) k_{i j} \\
\dot{\varepsilon}_{y y} & =-\dot{\varepsilon}_{x x} \\
\dot{\varepsilon}_{x y} & =\frac{T(y)-\sum_{i=1}^{m} \sum_{j=1}^{n} \frac{j-1}{i} x^{i} y^{j-2} k_{i j}}{2 \eta(x, y)} \\
= & Y(x, y)+\sum_{i=1}^{m} \sum_{j=1}^{n} b_{i j}(x, y) k_{i j}
\end{aligned}
$$


where the Newtonian viscosity is $\eta=1 /(2 \lambda)$ and

$$
\begin{aligned}
X(x, y) & =\frac{S(y)-x T^{\prime}(y)+\bar{\rho}_{y} g_{y} y-\bar{\rho}_{x} g_{x} x}{4 \eta(x, y)} \\
Y(x, y) & =\frac{T(y)}{2 \eta(x, y)} \\
a_{i j}(x, y) & =\frac{x^{i-1} y^{j-3}}{4 \eta(x, y)}\left[\frac{(j-1)(j-2)}{i(i+1)} x^{2}-y^{2}\right] \\
b_{i j}(x, y) & =-\frac{j-1}{2 i \eta(x, y)} x^{i} y^{j-2}
\end{aligned}
$$

The function $R(x, y)$ can also be expressed as a linear function of the coefficients $k_{i j}$ by combining equations (4.25) and (4.37), which leads to

$$
R(x, y)=K(x, y)+\sum_{i=1}^{m} \sum_{j=1}^{n} c_{i j}(x, y) k_{i j}
$$

where

$$
\begin{gathered}
K(x, y)=\int_{0}^{x} \frac{\partial X(\xi, y)}{\partial y} d \xi-\int_{0}^{y} \frac{\partial X(x, \tau)}{\partial x} d \tau-2 Y(x, y) \\
c_{i j}(x, y)=\int_{0}^{x} \frac{\partial a_{i j}(\xi, y)}{\partial y} d \xi-\int_{0}^{y} \frac{\partial a_{i j}(x, \tau)}{\partial x} d \tau-2 b_{i j}(x, y)
\end{gathered}
$$

Combining equations (4.38) and (4.36), one has

$$
\frac{\partial^{2} K(x, y)}{\partial x \partial y}+\sum_{i=1}^{m} \sum_{j=1}^{n} \frac{\partial^{2} c_{i j}(x, y)}{\partial x \partial y} k_{i j}=0
$$

which can be rewritten as 


$$
\sum_{i=1}^{m} \sum_{j=1}^{n} \frac{\partial^{2} c_{i j}(x, y)}{\partial x \partial y} k_{i j}=-\frac{\partial^{2} K(x, y)}{\partial x \partial y}
$$

This is a linear system with respect to the unknown constants $k_{i j}$, and therefore is numerically computable given the viscosity and the stress boundary conditions applied on the rear boundary of the wedge. Equations (4.39) can be applied to estimate numerically the spatial distribution of the function $C(x, y)$ for an orogenic wedge with Newtonian rheology. This can be done by the following procedure: first, assign reasonable large values to parameters $m$ and $n$ in equation (4.35); second, select a total of $m \times n$ points $P_{l}(l=1, \ldots, m \times n)$ evenly distributed throughout the wedge; third, use the coordinates of points $P_{l}\left(x_{l}, y_{l}\right)$ into equations (4.39), which forms a linear system of $m \times n$ equations with respect to a total of $m \times n$ coefficients $k_{i j}$; finally, determine $k_{i j}$ by solving this linear system of equations. When a sufficient number of points $P_{l}\left(x_{l}, y_{l}\right)$ is selected, the computed polynomial function $C(x, y)$ represents a first-degree approximation to the solution for the compatibility requirements (i.e., equations (4.24)). Once the function $C(x, y)$ is determined, stress, strain rate and velocity fields can be computed by equations (4.16), (4.20), and (4.34).

A function $C(x, y)$ computed by the above procedure is a general one because only the state of stress along the rear and the surface of the wedge have been specified (in terms of the functions $S(y)$ and $T(y)$ associated with equation (4.19)). Therefore, it may not necessarily match additional force and/or velocity boundary conditions along the wedge's basement. To find a special function $C(x, y)$ which matches these additional boundary conditions, additional constraints must be added to $C(x, y)$. In the following sub-section, these additional equations are derived; they also are linear systems with respect to the coefficients $k_{i j}$. 
Shear stress along the wedge basement

Shear resistance along the wedge basement is related to the state of stress through the following equation ( $c f$. Ranalli, 1995)

$$
\begin{aligned}
\tau_{b}= & \frac{1}{2}\left(\sigma_{y y}-\sigma_{x x}\right) \sin 2 \delta+\sigma_{x y} \cos 2 \delta \\
& =\frac{1}{2}\left(\sigma_{y y}-\sigma_{x x}\right) \sin 2\left(90^{\circ}-\theta\right)+\sigma_{x y} \cos 2\left(90^{\circ}-\theta\right) \\
& =\frac{1}{2}\left(\sigma_{y y}-\sigma_{x x}\right) \sin 2 \theta-\sigma_{x y} \cos 2 \theta
\end{aligned}
$$

where $\theta=\alpha+\beta$ is the sum of upper and lower slopes, and $\tau_{b}$ is the basal shear stress (see Fig. 4.2).

Substituting (4.35) and (4.16) into (4.40), one has

$$
\begin{aligned}
\tau_{b}(x, y)= & \frac{\sin 2 \theta}{2}\left[x T^{\prime}(y)-S(y)+\bar{\rho}_{x} g_{x} x-\bar{\rho}_{y} g_{y} y\right]-T(y) \cos 2 \theta \\
& -\frac{\sin 2 \theta}{2} \sum_{i=1}^{m} \sum_{j=1}^{n}\left(\frac{(j-1)(j-2)}{i(i+1)} x^{2}-y^{2}\right) x^{i-1} y^{j-3} k_{i j} \\
& +\cos 2 \theta \sum_{i=1}^{m} \sum_{j=1}^{n} \frac{j-1}{i} x^{i} y^{j-2} k_{i j}
\end{aligned}
$$

where $(x, y)$ are the coordinates along the wedge basement $y=b(x)$. Equation (4.41) can be written as

$$
\tau_{b}(x, y)=Z(x, y)+\sum_{i=1}^{m} \sum_{j=1}^{n} d_{i j}(x, y) k_{i j}
$$

where 


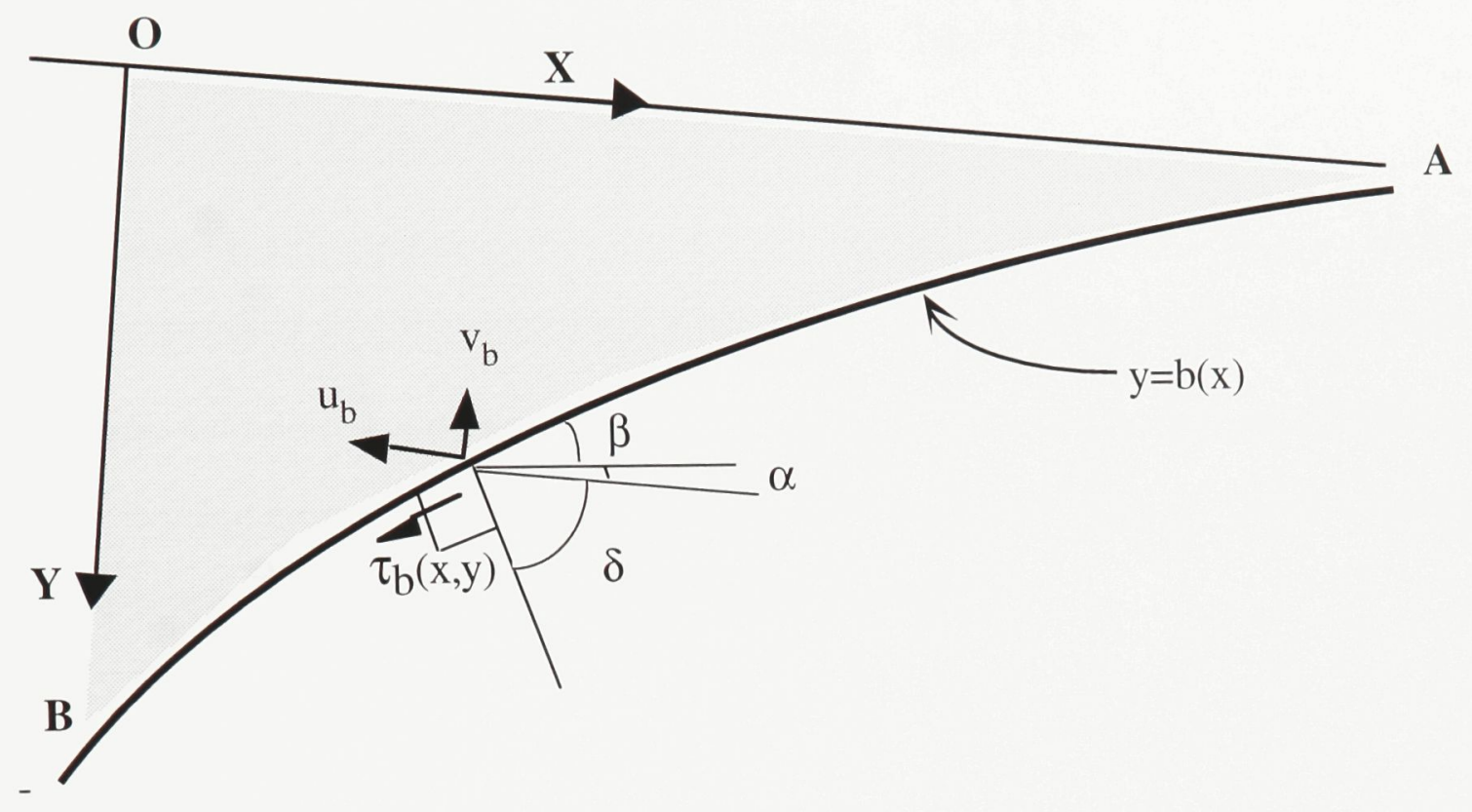

Figure 4.2 Boundary conditions along the basement $A B . \alpha$ and $\beta$ are the surface and basal slopes of the wedge, respectively; $\delta$ is the angle between the normal to the basement and the positive $x$-direction. In the given coordinate system, the geometry of the basement is represented by a function $y=b(x)$. The velocity at any basal point is represented by its two component $\left(u_{b}\right.$ and $\left.v_{b}\right) . \tau_{b}$ is the basal shear stress. 


$$
\begin{aligned}
& d_{i j}(x, y)=\frac{j-1}{i} x^{i} y^{j-2} \cos 2 \theta-\frac{\sin 2 \theta}{2}\left[\frac{(j-1)(j-2)}{i(i+1)} x^{2}-y^{2}\right] x^{i-1} y^{j-3} \\
& Z(x, y)=\frac{\sin 2 \theta}{2}\left[x T^{\prime}(y)-S(y)+\bar{\rho}_{x} g_{x} x-\bar{\rho}_{y} g_{y} y\right]-T(y) \cos 2 \theta
\end{aligned}
$$

Equations (4.42) represent the basal shear stress boundary conditions along the basement expressed as a linear system of equations with respect to the coefficients $k_{i j}$. Other kinds of force boundary conditions along the wedge basement, such as the Coulomb-Navier fracture criterion ( $c f$. Jaeger and Cook, 1969), can also be used and expressed as a linear system of equations with respect to $k_{i j}$.

\section{Velocity field}

To rewrite the velocity field in terms of the series expression for the function $C(x, y)$, equations (4.34) and (4.37) can be combined to give

$$
\begin{aligned}
u(x, y)= & u_{0}+\frac{y-y_{0}}{y_{1}-y_{0}}\left(u_{1}-u_{0}\right)+\frac{y-y_{1}}{y_{1}-y_{0}}\left[\int_{0}^{x_{0}} X\left(\xi, y_{0}\right) d \xi+\sum_{i=1}^{m} \sum_{j=1}^{n}\left(\int_{0}^{x_{0}} a_{i j}\left(\xi, y_{0}\right) d \xi\right) k_{i j}\right] \\
& -\frac{y-y_{0}}{y_{1}-y_{0}}\left[\int_{0}^{x_{1}} X\left(\xi, y_{1}\right) d \xi+\sum_{i=1}^{m} \sum_{j=1}^{n}\left(\int_{0}^{x_{1}} a_{i j}\left(\xi, y_{1}\right) d \xi\right) k_{i j}\right] \\
& +\frac{y-y_{0}}{y_{1}-y_{0}}\left[\int_{y_{0}}^{y_{1}} K\left(x_{0}, \tau\right) d \tau+\sum_{i=1}^{m} \sum_{j=1}^{n}\left(\int_{y_{0}}^{y_{1}} c_{i j}\left(x_{0}, \tau\right) d \tau\right) k_{i j}\right] \\
& +\int_{0}^{x} X(\xi, y) d \xi+\sum_{i=1}^{m} \sum_{j=1}^{n}\left(\int_{0}^{x} a_{i j}(\xi, y) d \xi\right) k_{i j}-\int_{y_{0}}^{y} K\left(x_{0}, \tau\right) d \tau-\sum_{i=1}^{m} \sum_{j=1}^{n}\left(\int_{y_{0}}^{y} c_{i j}\left(x_{0}, \tau\right) d \tau\right) k_{i j}
\end{aligned}
$$




$$
\begin{aligned}
v(x, y)= & v_{0}+\frac{x-x_{0}}{x_{1}-x_{0}}\left(v_{1}-v_{0}\right)-\frac{x-x_{1}}{x_{1}-x_{0}}\left[\int_{0}^{y_{0}} X\left(x_{0}, \tau\right) d \tau+\sum_{i=1}^{m} \sum_{j=1}^{n}\left(\int_{0}^{y_{0}} a_{i j}\left(x_{0}, \tau\right) d \tau\right) k_{i j}\right] \\
& +\frac{x-x_{0}}{x_{1}-x_{0}}\left[\int_{0}^{y_{1}} X\left(x_{1}, \tau\right) d \tau+\sum_{i=1}^{m} \sum_{j=1}^{n}\left(\int_{0}^{y_{1}} a_{i j}\left(x_{1}, \tau\right) d \tau\right) k_{i j}\right] \\
& +\frac{x-x_{0}}{x_{1}-x_{0}}\left[\int_{x_{0}}^{x_{1}} K\left(\xi, y_{0}\right) d \xi+\sum_{i=1}^{m} \sum_{j=1}^{n}\left(\int_{x_{0}}^{x_{1}} c_{i j}\left(\xi, y_{0}\right) d \xi\right) k_{i j}\right] \\
& -\int_{0}^{y} X(x, \tau) d \tau-\sum_{i=1}^{m} \sum_{j=1}^{n}\left(\int_{0}^{y} a_{i j}(x, \tau) d \tau\right) k_{i j}-\int_{x_{0}}^{x} K\left(\xi, y_{0}\right) d \xi-\sum_{i=1}^{m} \sum_{j=1}^{n}\left(\int_{x_{0}}^{x} c_{i j}\left(\xi, y_{0}\right) d \xi\right) k_{i j}
\end{aligned}
$$

These expressions for the velocity components can be rewritten in the following shorter form

$$
\begin{aligned}
& u(x, y)=U(x, y)+\sum_{i=1}^{m} \sum_{j=1}^{n} s_{i j}(x, y) k_{i j} \\
& v(x, y)=V(x, y)+\sum_{i=1}^{m} \sum_{j=1}^{n} t_{i j}(x, y) k_{i j}
\end{aligned}
$$

where

$$
\begin{aligned}
U(x, y) & =u_{0}+\frac{y-y_{0}}{y_{1}-y_{0}}\left(u_{1}-u_{0}\right)+\frac{y-y_{1}}{y_{1}-y_{0}} \int_{0}^{x_{0}} X\left(\xi, y_{0}\right) d \xi \\
& +\frac{y-y_{0}}{y_{1}-y_{0}}\left[-\int_{0}^{x_{1}} X\left(\xi, y_{1}\right) d \xi+\int_{y_{0}}^{y_{1}} K\left(x_{0}, \tau\right) d \tau\right]+\int_{0}^{x} X(\xi, y) d \xi-\int_{y_{0}}^{y} K\left(x_{0}, \tau\right) d \tau \\
V(x, y)= & v_{0}+\frac{x-x_{0}}{x_{1}-x_{0}}\left(v_{1}-v_{0}\right)-\frac{x-x_{1}}{x_{1}-x_{0}} \int_{0}^{y_{0}} X\left(x_{0}, \tau\right) d \tau \\
& +\frac{x-x_{0}}{x_{1}-x_{0}}\left[\int_{0}^{y_{1}} X\left(x_{1}, \tau\right) d \tau+\int_{x_{0}}^{x_{1}} K\left(\xi, y_{0}\right) d \xi\right]-\int_{0}^{y} X(x, \tau) d \tau-\int_{x_{0}}^{x} K\left(\xi, y_{0}\right) d \xi
\end{aligned}
$$




$$
\begin{aligned}
s_{i j}(x, y)= & \frac{y-y_{1}}{y_{1}-y_{0}} \int_{0}^{x_{0}} a_{i j}\left(\xi, y_{0}\right) d \xi+\frac{y-y_{0}}{y_{1}-y_{0}}\left[-\int_{0}^{x_{1}} a_{i j}\left(\xi, y_{1}\right) d \xi+\int_{y_{0}}^{y_{1}} c_{i j}\left(x_{0}, \tau\right) d \tau\right] \\
& +\int_{0}^{x} a_{i j}(\xi, y) d \xi-\int_{y_{0}}^{y} c_{i j}\left(x_{0}, \tau\right) d \tau \\
t_{i j}(x, y)= & -\frac{x-x_{1}}{x_{1}-x_{0}} \int_{0}^{y_{0}} a_{i j}\left(x_{0}, \tau\right) d \tau+\frac{x-x_{0}}{x_{1}-x_{0}}\left[\int_{0}^{y_{1}} a_{i j}\left(x_{1}, \tau\right) d \tau+\int_{x_{0}}^{x_{1}} c_{i j}\left(\xi, y_{0}\right) d \xi\right] \\
& -\int_{0}^{y} a_{i j}(x, \tau) d \tau-\int_{x_{0}}^{x} c_{i j}\left(\xi, y_{0}\right) d \xi
\end{aligned}
$$

Therefore, the components of the velocity field can also be expressed as linear functions of the coefficients $k_{i j}$.

The three important relations derived in this section are equations (4.39), (4.42), and (4.44). Given a Newtonian orogenic wedge with force boundary conditions at its rear "backstop" and force or velocity boundary conditions along its basement, the function $C(x, y)$ can be determined in principle, by numerically solving these three linear systems of equations. Once $C(x, y)$ is known, stresses, strain rates, and velocities can be determined. The focus in this thesis is on analytical solutions. Therefore, the following chapter considers another case which can be solved, namely, non-Newtonian rheology with some constraints on the deformation pattern of the wedge. 


\section{Chapter 5}

\section{A Linear Longitudinal Strain Rate Solution For Power-Law Rheology}

\subsection{Introduction}

As discussed in Chapter 4, a general analytical solution for stress, strain rate, and velocity for a two-dimensional power-law incompressible wedge can be obtained, but is extremely difficult to implement unless there are further simplifications and assumptions. In this chapter, two basic assumptions relating to the dynamics of orogenic wedges are used in order to derive a complete analytical solution for a wedge with general power-law rheology, namely, (1) the longitudinal strain rate varies linearly in the $x$-direction at any depth; and (2) the gradient in shear stress in the $x$-direction is negligible compared to the gradient of the vertical normal stress in the $y$-direction.

While it greatly simplifies both the derivation and the final expression of the solution, the first assumption allows the solution to simulate one of the most important features of thick-skinned orogenic wedge, i.e., syn-convergence extension ( $c f$. sections 2.6 and 5.1). The second assumption is in agreement with in situ stress measurements (McGarr and Gay, 1978) and has been adopted in numerous studies (Elliott, 1976; Boyer and Elliott, 1982; Davis et al., 1983; Dahlen, 1984; Suppe, 1985; Platt, 1986; Liu and Ranalli, 1992, 1994; Yin, 1986, 1988, 1989, 1993).

The linear longitudinal strain rate solution is then compared analytically and numerically to the classic one-dimensional solution derived by Nye (1957) for the movement of ice sheets, and it is shown to be a two-dimensional extension of the one-dimensional case. 
Some simple computational results are used to discuss the role of basal force and/or velocity boundary conditions in the stress state and deformation of a rectangular block with power-law rheology.

\subsection{Model geometry and rheology}

The two-dimensional geometry of the wedge and the associated coordinate system are identical to those used in Chapter 4 ( $c f$. Figure 4.1). The upper surface is a traction-free plane with topographic slope $\alpha$ dipping toward the front, while the basement dips from the front to the rear with slope angle $\beta$. The coordinate origin $O$ is fixed on the upper surface at the upper corner of the back of the wedge, with positive $x$-axis parallel to the surface and pointing towards the front, and positive $y$-axis perpendicular to the surface and pointing downwards.

As in Chapter 4, steady-state creep power-law rheology is assumed for the bulk rheology of the rock material in the wedge. All rheological parameters, such as the preexponential term $A$, the stress exponent $n$, and the activation energy $Q$, can vary with position, since they are functions of rock type and temperature.

Because of the identity of this chapter and Chapter 4 in terms of model geometry and bulk rheology, all the basic relations between strain rate and deviatoric stress and between velocity and strain rate remain unchanged ( $c f$. equations (4.1) to (4.10)).

There are basically four assumptions adopted in the derivation of the linear longitudinal power-law solution in this chapter. Among them, two fundamental assumptions have been mentioned in Section 5.1; the remaining two are related to the compressibility and the density of wedge material. These four assumptions are expressed by the following equations:

(1) the longitudinal strain rate $\left(\dot{\varepsilon}_{x x}\right)$ varies linearly in the longitudinal direction at any point within the wedge, i.e., 


$$
\frac{\partial^{2} \dot{\varepsilon}_{x x}}{\partial x^{2}}=0
$$

(2) the gradient of shear stress in the $x$-direction is negligible compared with the gradient of vertical normal stress in the $y$-direction

$$
\left|\frac{\partial \sigma_{x y}}{\partial x}\right|<\left|\frac{\partial \sigma_{y y}}{\partial y}\right|
$$

(3) the wedge material is incompressible, i.e., for plane strain

$$
\frac{\partial u}{\partial x}+\frac{\partial v}{\partial y}=0
$$

(4) the material density varies with depth only

$$
\frac{\partial \rho}{\partial x}=0
$$

The third assumption (equation 5.3) has been used in Chapter 4, and the fourth assumption (equation 5.4) is simply a first approximation to the usual density distribution in the upper crust of the Earth. Although it is greatly affected by the abundance of pore fluid (for a detailed discussion see Moore and Vrolijk, 1992), the density in orogenic wedges still varies mainly in the vertical direction because the porosity of the material does not change significantly in the longitudinal direction but varies mainly in the vertical direction ( $c f$. Zhao et al., 1986). 


\subsection{Derivation of the solution}

\subsubsection{Stress solution}

As derived in Chapter 4, a general stress solution to the equations of equilibrium (4.6) can be expressed as

$$
\begin{aligned}
& \sigma_{x x}=S(y)-x T^{\prime}(y)-\bar{\rho}_{x} g_{x} x+\int_{0}^{x} \int_{0}^{\varsigma} \frac{\partial^{2} C(\tau, y)}{\partial y^{2}} d \tau d \varsigma \\
& \sigma_{y y}=-\bar{\rho}_{y} g_{y} y+C(x, y) \\
& \sigma_{x y}=T(y)-\int_{0}^{x} \frac{\partial C(\tau, y)}{\partial y} d \tau
\end{aligned}
$$

Using equation (5.2), the function $C(x, y)$ can be easily proven to be zero. In other words, the assumption of negligible gradient of shear stress in the $x$-direction implies that the vertical normal stress $\sigma_{y y}$ is approximately lithostatic everywhere within the wedge. Because of the assumption about the density (equation (5.4)), we have $\bar{\rho}_{x}=\rho$ and the stress solution then becomes

$$
\begin{aligned}
& \sigma_{x x}=S(y)-x T^{\prime}(y)-\rho g_{x} x \\
& \sigma_{y y}=-\bar{\rho} g_{y} y \\
& \sigma_{x x}=T(y)
\end{aligned}
$$

where $\bar{\rho}$ is the depth-averaged density. In order to separate the stress due to gravity from that originating from non-gravitational tectonic forces, two new functions are introduced

$$
\begin{aligned}
& F(y)=S(y)+\bar{\rho} g_{y} y \\
& G(y)=T(y)+\bar{\rho} g_{x} y
\end{aligned}
$$


Combination of equations (5.6) and (5.7) leads to

$$
\begin{aligned}
& \sigma_{x x}=-\bar{\rho} g_{y} y+F(y)-x G^{\prime}(y) \\
& \sigma_{y y}=-\bar{\rho} g_{y} y \\
& \sigma_{x y}=-\bar{\rho} g_{x} y+G(y)
\end{aligned}
$$

which is the general stress solution to the equations of equilibrium (4.6), under the assumptions expressed by equations (5.2) and (5.4).

The state of stress along the rear vertical plane is obtained by setting $x=0$ in equations $(5.8)$

$$
\begin{aligned}
& \sigma_{x x}(0, y)=-\bar{\rho} g_{y} y+F(y) \\
& \sigma_{y y}(0, y)=-\bar{\rho} g_{y} y \\
& \sigma_{x y}(0, y)=-\bar{\rho} g_{x} y+G(y)
\end{aligned}
$$

Equations (5.9) show that the functions $F(y)$ and $G(y)$ are the non-gravitational (i.e., tectonic) normal and shear stress components applied longitudinally and vertically on the rear of the wedge (Figure 5.1).

\subsubsection{Strain rate solution}

As in Chapter 4, the strain rate solution can be derived from the relation between strain rate components and corresponding deviatoric stress components, i.e.,

$$
\begin{aligned}
& \dot{\varepsilon}_{x x}=\lambda \sigma_{x x}^{\prime}=\frac{\lambda}{2}\left[F(y)-x G^{\prime}(y)\right] \\
& \dot{\varepsilon}_{y y}=\lambda \sigma_{y y}^{\prime}=-\frac{\lambda}{2}\left[F(y)-x G^{\prime}(y)\right] \\
& \dot{\varepsilon}_{x y}=\lambda \sigma_{x y}^{\prime}=\lambda\left[G(y)-\bar{\rho} g_{x} y\right]
\end{aligned}
$$



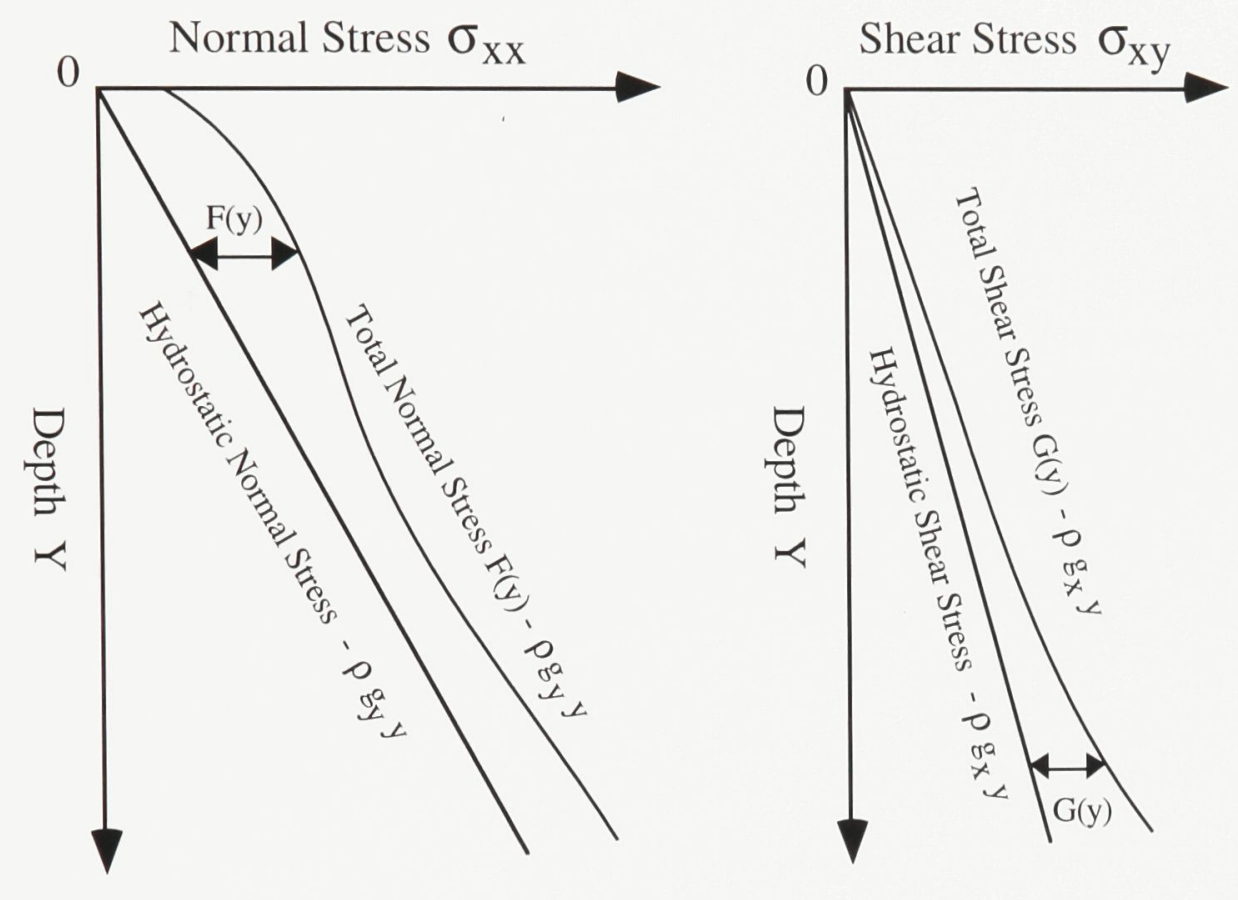

Figure 5.1 State of stress along the wedge rear boundary $(x=0)$. The stress axis refers to magnitude. According to the adopted sign convention, $F(y)<0$ when compressive; $G(y)<0$ when shearing downwards. 
where $\sigma_{x x}^{\prime}, \sigma_{y y}^{\prime}$ and $\sigma_{x y}^{\prime}$ are the deviatoric stress tensor components and the parameter $\lambda$, as defined in equation (4.4), can be expressed as in terms of the effective shear stress

$$
\begin{aligned}
& \tau_{E}=\left\{\frac{1}{4}\left[F(y)-x G^{\prime}(y)\right]^{2}+\left[G(y)-\bar{\rho} g_{x} y\right]^{2}\right\}^{\frac{1}{2}} \\
& \lambda=A \tau_{E}^{n-1}=A\left\{\frac{1}{4}\left[F(y)-x G^{\prime}(y)\right]^{2}+\left[G(y)-\bar{\rho} g_{x} y\right]^{2}\right\}^{\frac{n-1}{2}}
\end{aligned}
$$

\subsubsection{Velocity solution}

Since the wedge material is incompressible (i.e., equation (5.3)), a scalar stream function $\varphi(x, y)$ can be introduced to represent the longitudinal and vertical velocity components ( $c f$. e.g. Turcotte and Schubert, 1982; Lliboutry, 1987)

$$
u=\frac{\partial \varphi}{\partial y} \quad v=-\frac{\partial \varphi}{\partial x}
$$

where $u$ and $v$ are the velocity components in the $x$-and $y$-directions. Thus, the determination of the velocity vector field is reduced to that of the scalar function $\varphi(x, y)$.

Combination of equations (5.10), (5.11), (5.12) and (4.7) gives

$$
\begin{aligned}
& \frac{\partial^{2} \varphi}{\partial x \partial y}=\frac{\lambda}{2}\left[F(y)-x G^{\prime}(y)\right] \\
& \frac{\partial^{2} \varphi}{\partial y^{2}}-\frac{\partial^{2} \varphi}{\partial x^{2}}=2 \lambda\left[-\bar{\rho} g_{x} y+G(y)\right]
\end{aligned}
$$

This is the strain rate - stress relation expressed in terms of the stream function $\varphi$. 
To simplify the discussion, the assumption of linearity of longitudinal strain rate (equation 5.1) is expressed in terms of the scalar stream function $\varphi$, using equations (4.7) and (5.12), as

$$
\frac{\partial^{3} \varphi}{\partial x^{3}}=-p(x)
$$

where $p(x)$ is an arbitrary function of $x$. The minus sign before the function $p(x)$ is added to harmonize signs in the final strain rate and velocity equations (i.e., equations (5.29)) and therefore bears no substantial significance.

Differentiating the first of equations (5.13) twice with respect to $x$ and combining it with equation (5.14) leads to

$$
\frac{F(y)-x G^{\prime}(y)}{2} \frac{\partial^{2} \lambda}{\partial x^{2}}-G^{\prime}(y) \frac{\partial \lambda}{\partial x}=0
$$

Equation (5.15) is a second-order partial differential equation which can be transformed into a first-order equation and then solved by standard procedures ( $c f$. Spiegel, 1971). A general solution to equation (5.15) is

$$
\lambda=\frac{k(y)}{F(y)-x G^{\prime}(y)}+f_{0}(y)
$$

where $k(y)$ and $f_{0}(y)$ are two integration functions.

To determine these two integration functions, equations (5.16) and the second of equations (5.13) are combined to give

$$
\frac{\partial^{2} \varphi}{\partial y^{2}}-\frac{\partial^{2} \varphi}{\partial x^{2}}=2\left[\frac{k(y)}{F(y)-x G^{\prime}(y)}+f_{0}(y)\right]\left[-\bar{\rho} g_{x} y+G(y)\right]
$$


Differentiating equation (5.17) with respect to $x$ and combining it with equation (5.14) yields

$$
\frac{\partial^{3} \varphi}{\partial x \partial y^{2}}=-p(x)+\frac{2 k(y) G^{\prime}(y)}{\left[F(y)-x G^{\prime}(y)\right]^{2}}\left[-\bar{\rho} g_{x} y+G(y)\right]
$$

Again, differentiating equation (5.18) twice with respect to $x$ and applying equation (5.14) to the left-side of equation (5.18) gives

$$
-p^{\prime \prime}(x)+12 \frac{k(y)\left[G^{\prime}(y)\right]^{3}}{\left[F(y)-x G^{\prime}(y)\right]^{4}}\left[-\bar{\rho} g_{x} y+G(y)\right]=0
$$

The left side of equation (5.19) consists of two terms. The first term (i.e., $p^{\prime \prime}(x)$ ) is a function of $x$ only while the second term is a function of both $x$ and $y$. This means that both terms on the left side of the equation have to be two equal constants with opposite sign. Furthermore, it can be proven that these two constants are zero because the only $x$ variable of the second term appears explicitly in its denominator. Consequently, three cases can be derived from equation (5.19), that is,

$$
\begin{array}{ll}
k(y)=0 & p^{\prime \prime}(x)=0 \\
G^{\prime}(y)=0 & p^{\prime \prime}(x)=0 \\
G(y)=\bar{\rho} g_{x} y & p^{\prime \prime}(x)=0
\end{array}
$$


The second solution (equation 5.20b) represents a stress field corresponding to flow of a material compressed between two parallel plates (Nye, 1957), and is not applicable to an orogenic wedge with variable longitudinal deformation pattern. The third solution (equation $5.20 \mathrm{c}$ ) is not applicable either because it is a stress field with zero shear stress ( $\sigma_{x y}=0$ ) everywhere in the wedge, which is not able to produce any shear deformation. Therefore, only the first solution (equation 5.20a) is relevant.

Equations (5.19) and (5.20a) imply that

$$
p(x)=b_{1} x+b_{2}
$$

where $b_{1}$ and $b_{2}$ are two integration constants. Combining equations (5.21) and (5.14) it follows that

$$
\frac{\partial^{3} \varphi}{\partial x^{3}}=-b_{1} x-b_{2}
$$

that is, by integrating thrice with respect to $x$,

$$
\varphi(x, y)=-\frac{b_{1}}{24} x^{4}-\frac{b_{2}}{6} x^{3}+\frac{x^{2}}{2} f_{1}(y)+x f_{2}(y)+f_{3}(y)
$$

where $f_{i}(y)(i=1,2,3)$ are three integration functions. These can be determined by substituting equations (5.16) and (5.23) into the second of equations (5.13) 


$$
\begin{aligned}
\lambda & =f_{0}(y) \\
& =\frac{1}{2\left[-\bar{\rho} g_{x} y+G(y)\right]}\left(\frac{\partial u}{\partial y}+\frac{\partial v}{\partial x}\right) \\
& =\frac{1}{2\left[-\bar{\rho} g_{x} y+G(y)\right]}\left(\frac{\partial^{2} \varphi}{\partial y^{2}}-\frac{\partial^{2} \varphi}{\partial x^{2}}\right) \\
& =\frac{1}{2\left[-\bar{\rho} g_{x} y+G(y)\right]}\left\{\frac{1}{2}\left[f_{1}^{\prime \prime}(y)+b_{1}\right] x^{2}+\left[f_{2}^{\prime \prime}(y)+b_{2}\right] x-f_{1}(y)+f_{3}^{\prime \prime}(y)\right\}
\end{aligned}
$$

which implies that

$$
\begin{aligned}
& f_{1}^{\prime \prime}(y)=-b_{1} \\
& f_{2}^{\prime \prime}(y)=-b_{2} \\
& f_{3}^{\prime \prime}(y)=2\left[-\bar{\rho} g_{x} y+G(y)\right] f_{0}(y)+f_{1}(y)
\end{aligned}
$$

Consequently the functions $f_{i}(y)(i=1,2,3)$ are given by

$$
\begin{aligned}
& f_{1}(y)=-\frac{b_{1}}{2} y^{2}-a_{1} y-b_{3} \\
& f_{2}(y)=-\frac{b_{2}}{2} y^{2}-a_{2} y-b_{4} \\
& f_{3}(y)=2 \int_{0}^{y} \int_{0}^{\varsigma}\left[-\bar{\rho} g_{x} \tau+G(\tau)\right] f_{0}(\tau) d \tau d \varsigma-\frac{b_{1}}{24} y^{4}-\frac{a_{1}}{6} y^{3}-\frac{b_{3}}{2} y^{2}-a_{3} y-a_{4}
\end{aligned}
$$

where $a_{1}, a_{2}, a_{3}, a_{4}, b_{3}$ and $b_{4}$ are integration constants.

The functions $f_{0}(y), F(y)$ and $G(y)$ are not independent of each other. Their relation can be derived by applying equations (5.16), (5.23), and (5.26) into the first of equations $(5.12)$ 


$$
\begin{aligned}
\frac{\partial u}{\partial x} & =-x\left(a_{1}+b_{1} y\right)-a_{2}-b_{2} y \\
& =\frac{F(y)-x G^{\prime}(y)}{2} f_{0}(y)
\end{aligned}
$$

from which it follows that

$$
\begin{aligned}
f_{0}(y) & =-\frac{2\left(a_{2}+b_{2} y\right)}{F(y)} \\
G^{\prime}(y) & =-\frac{a_{1}+b_{1} y}{a_{2}+b_{2} y} F(y)
\end{aligned}
$$

Integrating the second of equations (5.28) with respect to $y$ gives

$$
G(y)=-\int_{0}^{y} \frac{a_{1}+b_{1} \tau}{a_{2}+b_{2} \tau} F(\tau) d \tau
$$

where the traction-free boundary condition on the surface of the wedge (i.e., $G(0)=0$ ) has been used to determine the integration constant. Equation (5.29) implies that, in the case of linear longitudinal strain rate, the normal and shear stress components of nongravitational stress along the rear boundary of the wedge are not independent of each other.

All unknown functions (i.e., $\lambda, \varphi, G$, and $\left.f_{i}(y)(i=1,2,3)\right)$ have been determined or expressed in terms of function $F$ and integration constants. Using equations (5.23), (5.26), (5.28), and (5.29) in combination with equations (5.6), (5.10), and (5.12), the complete general solution for stress, strain rate, and velocity in the case of linear longitudinal strain rate can be expressed as 


$$
\begin{aligned}
& \sigma_{x x}=-\bar{\rho} g_{y} y+\left(1+\frac{a_{1}+b_{1} y}{a_{2}+b_{2} y} x\right) F(y) \\
& \sigma_{y y}=-\bar{\rho} g_{y} y \\
& \sigma_{x y}=-\int_{0}^{y}\left[\rho g_{x}+\frac{a_{1}+b_{1} \tau}{a_{2}+b_{2} \tau} F(\tau)\right] d \tau \\
& \dot{\varepsilon}_{x x}=-\left(a_{1}+b_{1} y\right) x-\left(a_{2}+b_{2} y\right) \\
& \dot{\varepsilon}_{y y}=-\dot{\varepsilon}_{x x} \\
& \dot{\varepsilon}_{x y}=\frac{2\left(a_{2}+b_{2} y\right)}{F(y)} \int_{0}^{y}\left[\rho g_{x}+\frac{a_{1}+b_{1} \tau}{a_{2}+b_{2} \tau} F(\tau)\right] d \tau \\
& u=-\frac{x^{2}}{2}\left(a_{1}+b_{1} y\right)-x\left(a_{2}+b_{2} y\right)-\frac{b_{1}}{6} y^{3}-\frac{a_{1}}{2} y^{2}-b_{3} y-a_{3} \\
& +4 \int_{0}^{y}\left\{\frac{a_{2}+b_{2} \varsigma}{F(\varsigma)} \int_{0}^{\varsigma}\left[\rho g_{x}+\frac{a_{1}+b_{1} \tau}{a_{2}+b_{2} \tau} F(\tau)\right] d \tau\right\} d \varsigma \\
& v=\frac{b_{1}}{6} x^{3}+\frac{b_{2}}{2} x^{2}+x\left(\frac{b_{1}}{2} y^{2}+a_{1} y+b_{3}\right)+\frac{b_{2}}{2} y^{2}+a_{2} y+b_{4}
\end{aligned}
$$

The function $F(y)$ in the above equations is related to the rheology of the wedge material. Substituting equations (5.30a, b) into equations (4.3) and (4.4) gives

$$
-\frac{2\left(a_{2}+b_{2} y\right)}{F(y)}=A\left\{\frac{1}{4}\left[\left(1+\frac{a_{1}+b_{1} y}{a_{2}+b_{2} y} x\right) F(y)\right]^{2}+\left[\bar{\rho} g_{x} y+\int_{0}^{y} \frac{a_{1}+b_{1} \tau}{a_{2}+b_{2} \tau} F(\tau) d \tau\right]^{2}\right\}^{\frac{n-1}{2}}
$$

where the parameter $A=A_{0} \exp (-Q / R T)$ is a function of temperature and bulk rheology parameters $\left(A_{0}, Q\right)$ ( $c f$. equation 2.9). Equation (5.30d), together with equations (5.30a c), constitute a complete solution for a two-dimensional wedge with power-law rheology and linear longitudinal strain rate. 


\subsection{Implementation of analytical solution}

\subsubsection{General considerations}

Several observations about equations (5.30) should be noted here:

(1) There are two kinds of unknowns which are involved in equations (5.30), and need to be determined from boundary conditions: the function $F(y)$, and seven constants of integration (i.e., $a_{1}, a_{2} \ldots, b_{4}$ ). The function $F(y)$ is unknown since it is related to some of the unknown integration constants ( $c f$. equation 5.30d). According to their dynamical implications, these seven constants can be classified into two groups: those related to longitudinal strain rate $\left(a_{1}, a_{2}, b_{1}\right.$, and $\left.b_{2}\right)$, and those related to rigid-body motion corresponding to the velocity and rotation rate at the origin $\left(a_{3}, b_{3}\right.$, and $\left.b_{4}\right)$. These unknown function and constants are listed in Table 5.1.

Table 5.1 Physical significance of function and constants appearing in equations (5.30)

\begin{tabular}{cl}
\hline$F(y)$ & tectonic normal stress at the rear of the wedge $(x=0)$ \\
\hline$a_{1}, a_{2}$ & longitudinal strain rate on top surface $(y=0)$ \\
\hline$b_{1}, b_{2}$ & vertical gradient of longitudinal strain rate \\
\hline$a_{3}, b_{4}$ & velocity at the origin $(x=y=0)$ \\
\hline$b_{3}$ & rigid-body rotation rate \\
\hline
\end{tabular}

(2) The state of stress at the rear of the wedge $(x=0)$, represented by the functions $F(y)$ and $G(y)$, is related to the rheology and the strain rate of the wedge. This is indicated by two factors: (a) $G(y)$ is related to $F(y)$ and the strain rate by equation (5.29); and (b) $F(y)$ is a function of the rheology, the temperature distribution, and the longitudinal strain rate of the wedge(i.e., equation 5.30d).

(3) There are two possible ways to deal with equation (5.30d): (a) computation of the strain rate for a given wedge rheology and independently specified $F(y)$; and (b) 
computation of $F(y)$ for a given wedge rheology and strain rate (independently specified or determined by other means). The first way is practically not implementable, since it is very likely that the specified $F(y)$ would not be compatible with the given rheology and temperature distribution (because the four constants $a_{1}, a_{2}, b_{1}$, and $b_{2}$ can be determined by applying the coordinates of any four points within the wedge into equation (5.30d) which results in a system of four equations; but equation (5.30d) with these constants may not be satisfied throughout the whole wedge). The second way, on the other hand, allows determination of the tectonic normal stress required at the rear of a wedge with given material rheology and temperature distribution to maintain a given longitudinal strain rate. This approach is used in all the computations reported in this thesis.

(4) Additional constraints on the strain rate and the rheology of the wedge are introduced by equation $(5.30 \mathrm{~d})$ : the left side of the equation is a function of $y$ only while the right side of the equation may vary with both $x$ and $y$. Obviously this kind of additional constraint is caused by the assumption of linear longitudinal strain rate (i.e., equation 5.1) which eventually requires the parameter $\lambda$ to be a function of $y$ only (see equation 5.24). The effect of this additional constraint, however, can be reasonably minimized if the equations (5.30) are applied to a short segment of the wedge so that the right side of equation (5.30d) does not vary significantly in $x$ direction. (It has been observed that, for a typical wedge segment with a longitudinal width less than $10 \mathrm{~km}$, the longitudinal variation of the computed function $F(y)$ is less than $5 \%$.)

\subsubsection{Wedge segmentation}

The last observation mentioned above emphasizes the need to subdivide the wedge into a number of segments, with two neighbouring segments linked by a thin boundary layer parallel to the $y$-direction (see Figure 5.2). Within each segment, a set of constants (i.e., $\left.a_{1}, a_{2} \ldots, b_{4}\right)$ and the function $F(y)$ can be computed from appropriate force and/or 
velocity boundary conditions, and consequently the stress, strain rate, and velocity fields can be found. The stress, strain rate, and velocity fields in the thin boundary layer between two neighbouring segments can be found by linear interpolation (of course, boundary conditions specified for the two neighbouring segments must be compatible). Stress, strain rate, and velocity solutions computed for a segmented wedge are a good first approximation to a continuous wedge if the wedge is subdivided into a sufficient number of short segments.

\subsubsection{Mutual dependence of the function $F(y)$ and the strain rate field}

Within each wedge segment, where longitudinal strain rate is a linear function in the $x$ direction, the main difficulty in applying the solution given by equations (5.30) derives from the interdependence of function $F(y)$ and longitudinal strain rate (i.e., $a_{1}, a_{2}, b_{1}$, and $\left.b_{2}\right)$. Computation of $F(y)$ from equation $(5.30 \mathrm{~d})$ requires known values of longitudinal strain rate $\left(a_{1}, a_{2}, b_{1}\right.$, and $\left.b_{2}\right)$; on the other hand, the computation of the constants of integration requires knowledge of the function $F(y)(c f$. equations $5.30 \mathrm{a}-\mathrm{c})$. This is, of course, only a computational difficulty. Physically, the interdependence of tectonic stress and longitudinal strain rate is simply a consequence of the rheological properties of the wedge.

In order to overcome this computational deadlock between the function $F(y)$ and the longitudinal strain rate field, other conditions must be specified in advance. Specification of the function $F(y)$ is not possible as explained in the previous subsection. Therefore, one must assign sufficient constraints on the spatial distribution of longitudinal strain rate and then compute $F(y)$. Once $F(y)$ is determined, the remaining constants $a_{3}, b_{3}$, and $b_{4}$ 


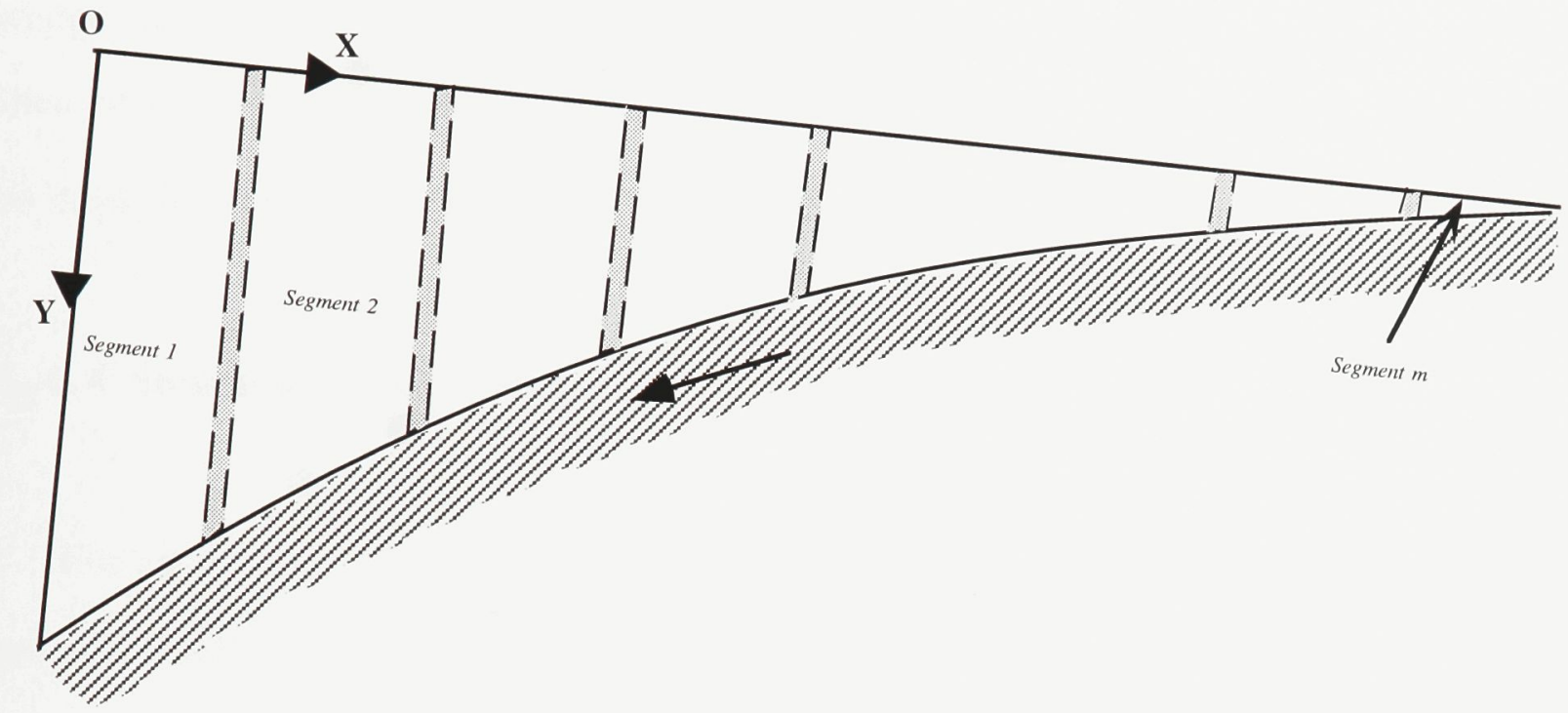

Figure 5.2 Subdivision of the wedge into a series of short segments. See text for details. 
(the second group of constants of Table 5.1) can be determined from velocity boundary conditions.

The reasonableness of the assigned spatial constraints on longitudinal strain rate can be tested by the smoothness of solution (for stress, strain rate and velocity) in the whole wedge, and by considering the state of stress along the wedge basement, i.e. the basal shear stress $\tau_{b}$. This should be reasonably smooth, increase from the front to the rear, and be in the 0 - $100 \mathrm{MPa}$ range ( $c f$. section 2.4 for detailed discussion).

\subsubsection{Solution implementation}

For each wedge segment $i(i=1, \ldots, m)$, the following two sets of boundary conditions are specified (Figure 5.3): three points on the top boundary with assigned velocity $(u, v)$ and one point on the basement with assigned basal shear stress $\left(\tau_{b}\right)$. Within the segment a local coordinate system is defined with origin at the top left corner and local $x$ and $y$ axes parallel to the global axes as defined in Figure 4.1. Applying the velocity boundary conditions to equation $(5.30 \mathrm{c})$, one has

$$
\begin{aligned}
& -\frac{x_{k}^{2}}{2} a_{1}-x_{k} a_{2}-a_{3}=u_{k} \\
& \frac{x_{k}^{3}}{6} b_{1}+\frac{x_{k}^{2}}{2} b_{2}+b_{3} x_{k}+b_{4}=v_{k}
\end{aligned}
$$

where $\left(u_{k}, v_{k}\right)$ are the velocity components assigned at points $\left(x_{k}, 0\right)(k=1,2,3)$.

The constants $a_{1}, a_{2}$, and $a_{3}$ are computed from the first of equations (5.31). This constrains the longitudinal strain rate along the top surface. An additional equation is needed to compute $b_{j}(j=1,2,3,4)$ from the second of equations (5.31). This additional relation is provided by the specification of shear stress at one point along the lower boundary of the wedge segment (see Figure 5.3) 


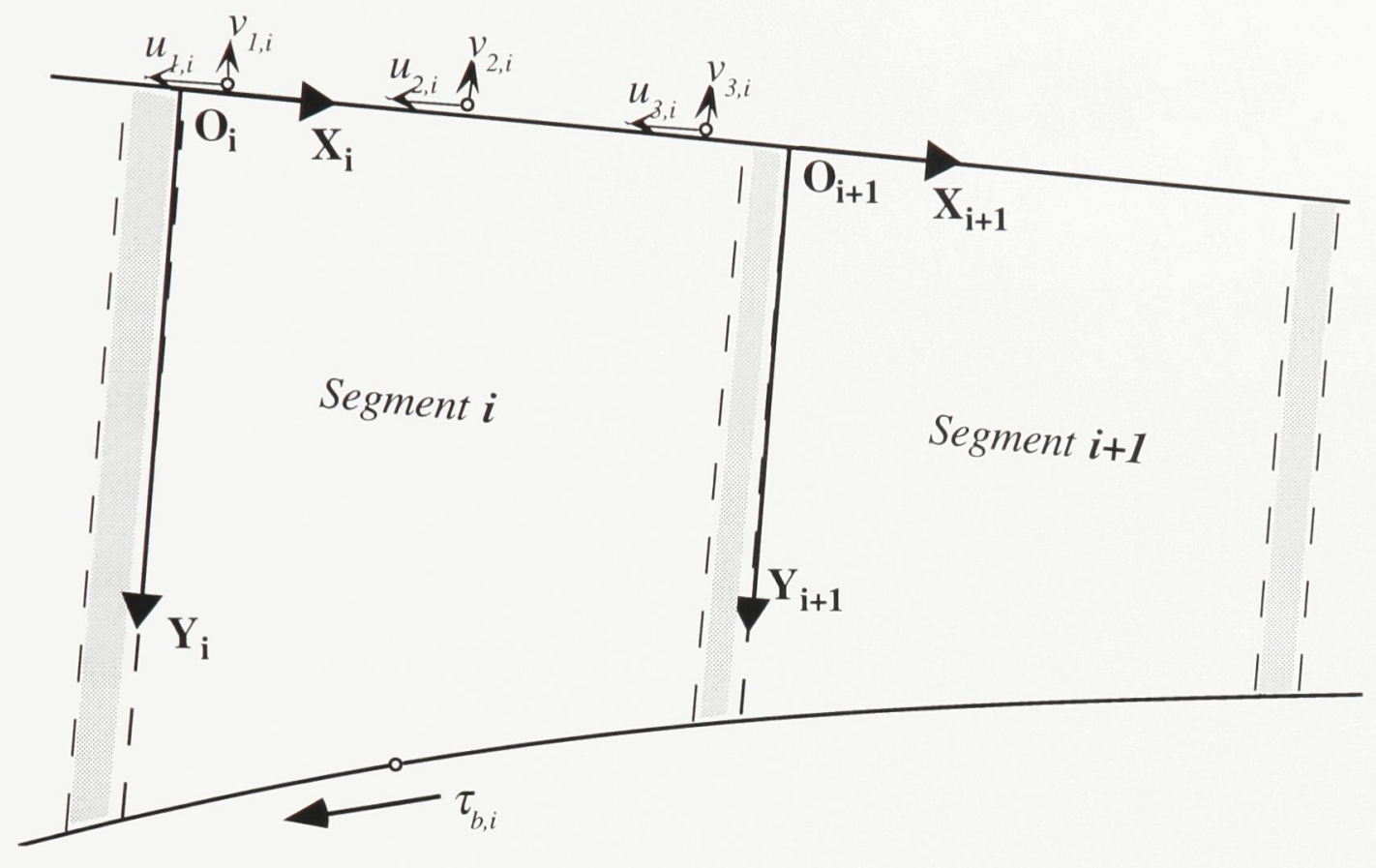

Figure 5.3 A short segment of wedge with assigned velocity boundary conditions on top and basal shear stress on bottom. Within the segment a local coordinate system is defined with origin at the top left corner. See text for details. 


$$
\begin{aligned}
\tau_{b} & =\frac{1}{2}\left(\sigma_{y y}-\sigma_{x x}\right) \sin 2 \theta_{b}-\sigma_{x y} \cos 2 \theta_{b} \\
& =-\frac{1}{2}\left(1+\frac{a_{1}+b_{1} y_{b}}{a_{2}+b_{2} y_{b}} x_{b}\right) F\left(y_{b}\right) \sin 2 \theta_{b}+\cos 2 \theta_{b} \int_{0}^{y_{b}}\left[\rho g_{x}+\frac{a_{1}+b_{1} \tau}{a_{2}+b_{2} \tau} F(\tau)\right] d \tau
\end{aligned}
$$

where the basal shear stress $\tau_{b}$ is defined at the point $\left(x_{b}, y_{b}\right)$, and $\theta_{b}$ is the sum of top and basal slope angles at that point. Unfortunately, equation (5.32) can not be used directly since the function $F(y)$ is invoked in this equation. To solve this dilemma, a simple linear relation between the constant $b_{1}$ and the top longitudinal strain rate at the central point of the segment is assumed

$$
b_{1}=\gamma\left(a_{2}+a_{1} w\right)
$$

where $w$ is half the width of the wedge segment and the parameter $\gamma$ is a constant. Together with the second of equations (5.31), equation (5.33) is initially used with a trial value for the parameter $\gamma$ to compute $b_{j}(j=1,2,3,4)$. The correct value for $\gamma$ is found by examining the convergence of the computed basal shear stress $\left(\tau_{b}^{*}\right)$ and the assigned basal shear stress $\left(\tau_{b}\right)$.

\subsubsection{Flowchart and numerical considerations}

A flowchart describing the main steps in the solution of equations (5.30) is shown in Figure 5.4.

Equation (5.30d) is a complicated nonlinear function of $F(y)$, and computation of $F(y)$ is very sluggish if equation (5.30d) is used directly. Also, there exists a singular point at depth $y_{s}=-a_{2} / b_{2}$. To avoid these problems, the following modifications of equation (5.30d) are implemented: 


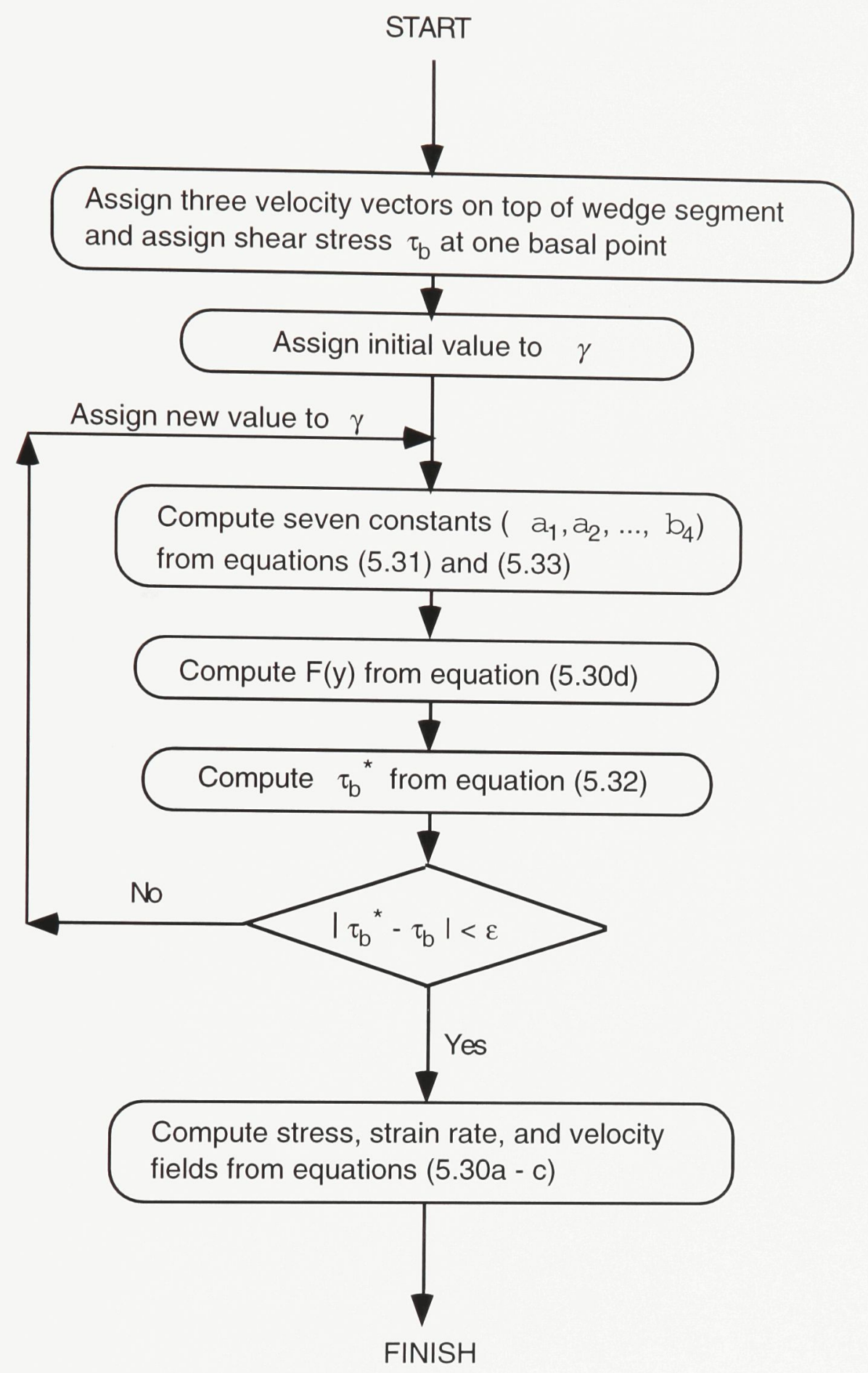

Figure 5.4 A simplified flowchart for the implementation of the solution to equations (5.30) for each individual wedge segment ( $\varepsilon$ is a pre-assigned small positive value). 
(1) Introducing an effective viscosity function $\eta(y)$, equation (5.30d) is rewritten as

$$
\frac{1}{2 \eta(y)}=\tilde{A}\left\{4\left[a_{2}+b_{2} y+\left(a_{1}+b_{1} y\right) w\right]^{2} \eta(y)^{2}+\left[\int_{0}^{y}\left[\rho(\tau) g_{x}-4\left(a_{1}+b_{1} \tau\right) \eta(\tau)\right] d \tau\right]^{2}\right\}^{\frac{\tilde{n}-1}{2}}
$$

where $w$ is half the width of each segment and $\tilde{A}(y)=A(w, y)$ and $\tilde{n}(y)=n(w, y)$. The function $\eta(y)=-F(y) / 4\left(a_{2}+b_{2} y\right)$ is the effective viscosity of the wedge segment and it becomes the real Newtonian viscosity when the stress exponent of bulk rheology equals unity.

(2) Differentiation of equation (5.34) with respect to $y$ yields

$$
\frac{d \eta(y)}{d y}=\frac{h_{0}(y)+h_{1}(y) s(y)}{h_{2}(y)}
$$

where

$$
\begin{aligned}
& h_{0}(y)=4\left(b_{2}+b_{1} w\right)\left[a_{2}+b_{2} y+\left(a_{1}+b_{1} y\right) w\right] \eta^{2}-\frac{(2 \tilde{A} \eta)^{\frac{2}{1-\tilde{n}}}}{1-\tilde{n}}\left[\frac{1}{\tilde{A}} \frac{d \tilde{A}}{d y}+\frac{\ln (2 \tilde{A} \eta)}{1-\tilde{n}} \frac{d \tilde{n}}{d y}\right] \\
& h_{1}(y)=\rho g_{x}-4\left(a_{1}+b_{1} y\right) \eta \\
& h_{2}(y)=\frac{(2 \tilde{A} \eta)^{\frac{2}{1-\tilde{n}}}}{(1-\tilde{n}) \eta}-4\left[a_{2}+b_{2} y+\left(a_{1}+b_{1} y\right) w\right]^{2} \eta \\
& s(y)=\int_{0}^{y}\left[\rho(\tau) g_{x}-4\left(a_{1}+b_{1} \tau\right) \eta(\tau)\right] d \tau
\end{aligned}
$$

Equation (5.35a) can be integrated numerically (using the Runge-Kutta integration method with adaptive step-size control ; Press et al., 1992). The numerical computation of the 
function $s(y)$ is done by dividing the total integration range into a series of small subranges within which integration is approximated by the trapezoidal rule (Kreyszig, 1988).

(3) The boundary value $\eta_{0}(=\eta(0))$ of the viscosity function is computed by setting $y=0$ in equation $(5.34)$

$$
\eta(0)=\frac{\mid a_{2}+a_{1} w w^{\frac{1}{\tilde{n}(0)}}-1}{2 \tilde{A}(0)^{\frac{1}{\tilde{n}(0)}}}
$$

Equation (5.30d) (see Figure 5.4) is therefore replaced by the combination of equations (5.34), (5.35), and (5.36) to compute the function $F(y)$ through the effective viscosity function $\eta(y)$.

The solution given by equations $(5.30 \mathrm{a}-\mathrm{c})$ can be conveniently expressed in terms of the effective viscosity function $\eta(y)$ as

$$
\begin{aligned}
& \sigma_{x x}=-g_{y} \int_{0}^{y} \rho(\tau) d \tau-4\left[a_{2}+b_{2} y+\left(a_{1}+b_{1} y\right) x\right] \eta(y) \\
& \sigma_{y y}=-g_{y} \int_{0}^{y} \rho(\tau) d \tau \\
& \sigma_{x y}=-\int_{0}^{y}\left[\rho(\tau) g_{x}-4\left(a_{1}+b_{1} \tau\right) \eta(\tau)\right] d \tau \\
& \dot{\varepsilon}_{x x}=-\left(a_{1}+b_{1} y\right) x-\left(a_{2}+b_{2} y\right) \\
& \dot{\varepsilon}_{y y}=-\dot{\varepsilon}_{x x} \\
& \dot{\varepsilon}_{x y}=-\frac{1}{2 \eta(y)} \int_{0}^{y}\left[\rho(\tau) g_{x}-4\left(a_{1}+b_{1} \tau\right) \eta(\tau)\right] d \tau
\end{aligned}
$$




$$
\begin{aligned}
u= & -\frac{x^{2}}{2}\left(a_{1}+b_{1} y\right)-x\left(a_{2}+b_{2} y\right)-\frac{b_{1}}{6} y^{3}-\frac{a_{1}}{2} y^{2}-b_{3} y-a_{3} \\
& -\int_{0}^{y}\left\{\frac{1}{\eta(\varsigma)} \int_{0}^{\varsigma}\left[\rho(\tau) g_{x}-4\left(a_{1}+b_{1} \tau\right) \eta(\tau)\right] d \tau\right\} d \varsigma \\
v & =\frac{b_{1}}{6} x^{3}+\frac{b_{2}}{2} x^{2}+x\left(\frac{b_{1}}{2} y^{2}+a_{1} y+b_{3}\right)+\frac{b_{2}}{2} y^{2}+a_{2} y+b_{4}
\end{aligned}
$$

\subsection{Comparison between two-dimensional and one-dimensional solutions}

The above linear longitudinal strain rate solution is a two-dimensional extension of the classic one-dimensional solution for glacier flow (Nye, 1957). In this section this statement is proven by a detailed comparison between the two solutions, including computational results.

It is obvious from Table 5.2 that the solution obtained in this chapter is a twodimensional extension of Nye's classic one-dimensional solution. The present solution reduces to Nye's solution for $a_{1}=b_{1}=b_{2}=b_{3}=0$, which is equivalent to the fourth assumption used in Nye's analysis (i.e., $\partial v / \partial x=0$ ). In order to obtain the solution in the one-dimensional case, gravity was introduced into a solution given by Prandtl in 1923 ( $c f$. review by Nádai, 1931) for the compression of a rigid-plastic weightless layer (Nye, 1951), which results in a stress tensor independent of longitudinal location. The less restrictive assumption that the longitudinal gradient in horizontal shear stress is negligible compared with the vertical gradient in vertical normal stress results in a horizontal normal deviatoric stress which is a linear function of the longitudinal location. Through segmentation of the wedge, this allows to accommodate a much more complex velocity field. 
Table 5.2 Comparison between Nye's one-dimensional solution and the present two-dimensional solution

1-D theory* (Nye, 1957)

present 2-D solution

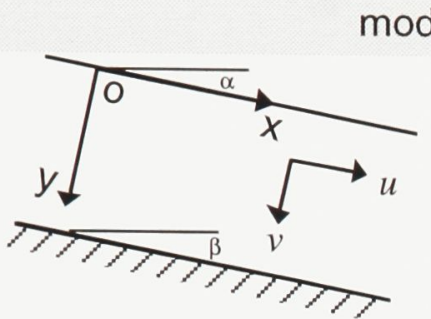

model geometry and rheology

The basement is parallel to the top surface $(\alpha=\beta)$; power-law rheology is postulated as a function of depth $y$ only

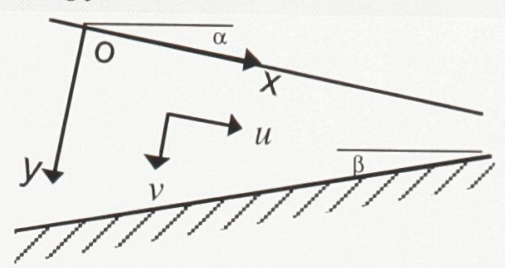

The basement and top surface dip in opposite directions; power-law rheology vary both longitudinally and vertically

\section{model assumptions}

(1) incompressible plane strain flow;

(2) stress tensor is function of depth only;

(1) incompressible plane strain flow;

(3) density is function of depth only;

(4) $\partial v / \partial x=0$

(2) $\partial \sigma_{x y} / \partial x<<\partial \sigma_{y y} / \partial y$;

(3) density is function of depth only;

(4) $\partial^{2} \dot{\varepsilon}_{x x} / \partial x^{2}=0$

$$
\begin{aligned}
& \sigma_{x x}=-\bar{\rho} g_{y} y+F(y) \\
& \sigma_{y y}=-\bar{\rho} g_{y} y \\
& \sigma_{x y}=-\bar{\rho} g_{x} y \\
& -\frac{2 a_{2}}{F(y)}=A^{-n} \tau_{E}^{\prime n-1}
\end{aligned}
$$

\section{stress solution}

$$
\begin{aligned}
& \sigma_{x x}=-\bar{\rho} g_{y} y+\left[1+\frac{a_{1}+b_{1} y}{a_{2}+b_{2} y} x\right] F(y) \\
& \sigma_{y y}=-\bar{\rho} g_{y} y \\
& \sigma_{x y}=-\bar{\rho} g_{x} y-\int_{0}^{y} \frac{a_{1}+b_{1} y}{a_{2}+b_{2} y} F(\tau) d \tau \\
& -\frac{2\left(a_{2}+b_{2} y\right)}{F(y)}=A \tau_{E}^{\prime n-1}
\end{aligned}
$$

\section{strain rate solution}

$$
\begin{aligned}
& \dot{\varepsilon}_{x x}=-a_{2} \\
& \dot{\varepsilon}_{y y}=a_{2} \\
& \dot{\varepsilon}_{x y}=\frac{2 a_{2} \bar{\rho} g_{x} y}{F(y)}
\end{aligned}
$$

$$
\begin{aligned}
& \dot{\varepsilon}_{x x}=-\left(a_{1}+b_{1} y\right) x-\left(a_{2}+b_{2} y\right) \\
& \dot{\varepsilon}_{y y}=\left(a_{1}+b_{1} y\right) x+\left(a_{2}+b_{2} y\right) \\
& \dot{\varepsilon}_{x y}=\frac{2\left(a_{2}+b_{2} y\right)}{F(y)}\left[\bar{\rho}_{g_{x}} y+\int_{0}^{y} \frac{a_{1}+b_{1} \tau}{a_{2}+b_{2} \tau} F(\tau) d \tau\right]
\end{aligned}
$$

\section{velocity solution}

$$
\begin{aligned}
& u=-a_{2} x+4 a_{2} g_{x} \int_{0}^{y} \frac{\bar{\rho} \tau}{F(\tau)} d \tau-a_{3} \\
& v=a_{2} y+b_{4}
\end{aligned}
$$

$$
\begin{aligned}
& u=-\frac{a_{1}}{2}\left(x^{2}+y^{2}\right)-a_{2} x-a_{3}-\frac{b_{1}}{2} y\left(x^{2}+\frac{y^{2}}{3}\right)-b_{2} x y \\
& -b_{3} y+4 \int_{0}^{y}\left\{\frac{a_{2}+b_{2} \varsigma}{F(\varsigma)}\left[\bar{\rho}_{g_{x}} \varsigma+\int_{0}^{\zeta} \frac{a_{1}+b_{1} \tau}{a_{2}+b_{2} \tau} F(\tau) d \tau\right]\right\} d \varsigma \\
& v=a_{1} x y+a_{2} y+\frac{b_{1}}{2} x\left(\frac{x^{2}}{3}+y^{2}\right)+\frac{b_{2}}{2}\left(x^{2}+y^{2}\right)+b_{3} x+b_{4}
\end{aligned}
$$

* Minor modifications to Nye's original expressions have been made to facilitate comparison. 


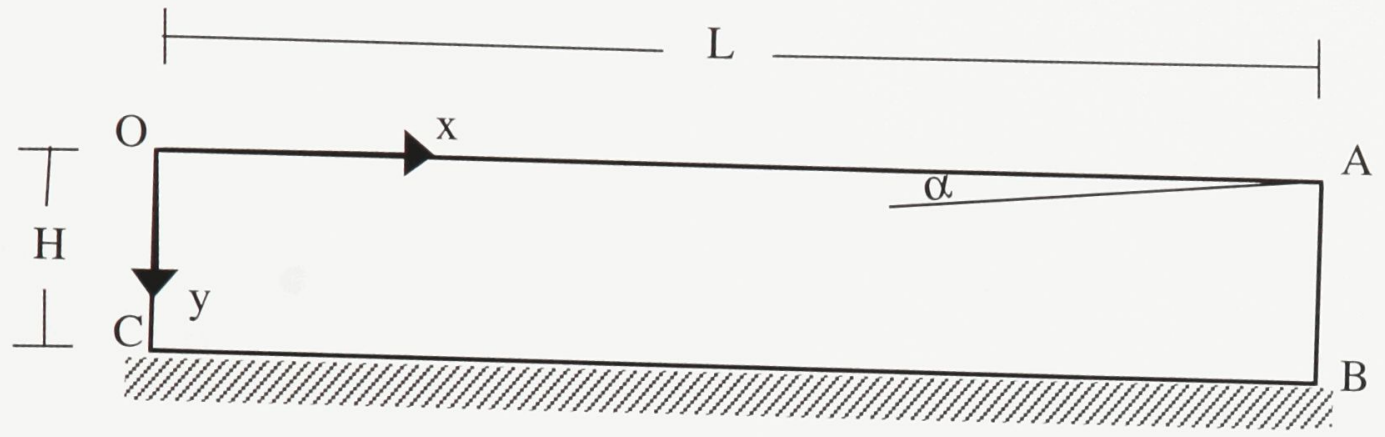

Figure 5.5 Parallel-sided rectangular block with power-law rheology and basement slope $\alpha$. 
Table 5.3 Values of model parameters for rectangular block with length $L=250 \mathrm{~km}$, depth $H=40 \mathrm{~km}$ and constant density $\rho=2800 \mathrm{~kg} / \mathrm{m}^{3}$ over a basement with slope angle $\alpha=3^{\circ}$

\begin{tabular}{lcccccc} 
& \multicolumn{7}{c}{ Case* } \\
\cline { 2 - 6 } Parameter & $\mathrm{A}$ & $\mathrm{B}$ & $\mathrm{C}$ & $\mathrm{D}$ & $\mathrm{E}$ & $\mathrm{F}$ \\
\hline$u_{0}\left(\times 10^{-10} \mathrm{~m} \mathrm{~s}^{-1}\right)$ & $31.71 * *$ & 31.71 & 31.71 & 31.71 & 31.71 & 31.71 \\
$u_{1}\left(\times 10^{-15} \mathrm{~s}^{-1}\right)$ & -1 & -1 & -1 & -1 & -1 & -1 \\
$u_{2}\left(\times 10^{-20} \mathrm{~m}^{-1} \mathrm{~s}^{-1}\right)$ & 0 & 0 & -1.8 & 0 & 0 & 0 \\
$v_{0}\left(\times 10^{-10} \mathrm{~m} \mathrm{~s}^{-1}\right)$ & -0.4 & -0.4 & -0.4 & -0.4 & -0.4 & -0.4 \\
$v_{1}\left(\times 10^{-15} \mathrm{~s}^{-1}\right)$ & 0 & 0 & 0 & -4 & 0 & 0 \\
$\tau_{0}(\mathrm{MPa})$ & -57.44 & -57.44 & -57.44 & -57.44 & -57.44 & -57.44 \\
$\tau_{1}\left(\mathrm{MPa} \mathrm{km} \mathrm{km}^{-1}\right)$ & 0 & 0 & 0.068 & 0 & 0 & 0 \\
$T_{0}(\mathrm{~K})$ & $\S$ & & & & 750 & 750 \\
$T_{1}\left(\mathrm{~K} \mathrm{~km}{ }^{-1}\right)$ & & & & & 0 & 2.5 \\
$A_{0}^{*}\left(\times 10^{-23} \mathrm{~Pa}^{-1} \mathrm{~s}^{-1}\right)$ & 5 & 5 & 5 & 5 & & anorthosite $\dagger$ \\
$A_{1}^{*}\left(\times 10^{-23} \mathrm{~Pa}^{-1} \mathrm{~s}^{-1} \mathrm{~km}^{-1}\right)$ & 0 & 1.125 & 0 & 0 & & \\
power-law rheology & & & & & & \\
\hline
\end{tabular}

* Cases A, B, C, D, E, and F refer to Figures 5.6, 5.7, 5.8, 5.9, 5.10, and 5.11
A: Newtonian, constant $\eta\left(=10^{22} \mathrm{~Pa} \mathrm{~s}\right)$, constant $\dot{\varepsilon}_{x x}$ and constant $v$ on surface, constant $\tau_{b}$
B: Newtonian, depth-dependent $\eta$, constant $\dot{\varepsilon}_{x x}$ and constant $v$ on surface, constant $\tau_{b}$
C: Newtonian, constant $\eta\left(=10^{22} \mathrm{~Pa} \mathrm{~s}\right.$ ), variable $\dot{\varepsilon}_{x x}$ and constant $v$ on surface, variable $\tau_{b}$
D: Newtonian, constant $\eta\left(=10^{22} \mathrm{~Pa} \mathrm{~s}\right)$, constant $\dot{\varepsilon}_{x x}$ and variable $v$ on surface, constant $\tau_{b}$
E: Power-law, constant $T$, constant $\dot{\varepsilon}_{x x}$ and constant $v$ on surface, constant $\tau_{b}$
F: Power-law, depth-dependent $T$, constant $\dot{\varepsilon}_{x x}$ and constant $v$ on surface, constant $\tau_{b}$
** $\quad 100 \mathrm{~mm} \mathrm{a}^{-1} \approx 31.71 \times 10^{-10} \mathrm{~m} \mathrm{~s}^{-1}$
$\S$ empty entry indicates that parameter is not applicable
$\dagger$ creep parameters: $A_{0}=2.06 \times 10^{-23}\left(\mathrm{~Pa}^{-3.2} \mathrm{~s}^{-1}\right), n=3.2, Q=238\left(\mathrm{~kJ} \mathrm{~mol}^{-1}\right)$

In order to illustrate this point, the solution given by equations (5.30) is applied to the analysis of a rectangular block with various rheological parameters and boundary conditions (see Figure 5.5). The parameter values used in the computations are listed in Table 5.3. All cases, for simplicity, are parameterized in the following way: 
(a) The velocity boundary conditions along the top free surface are parabolic and linear functions of position, respectively, that is

$$
\begin{aligned}
& u_{\text {top }}=u_{0}+u_{1} x+u_{2} x^{2} \\
& v_{\text {top }}=v_{0}+v_{1} x
\end{aligned}
$$

where $u_{0}, u_{1}, u_{2}, v_{0}$, and $v_{1}$ are constants.

(b) The basal shear stress along the block basement is a linear function of position

$$
\tau_{b}=\tau_{0}+\tau_{1} x
$$

where $\tau_{0}$ and $\tau_{1}$ are constants.

(c) The temperature within the block varies with depth only and is represented by a linear function

$$
T=T_{0}+T_{1} y
$$

where $T_{0}$ and $T_{1}$ are constants.

(d) The rheology of the block can be nonlinear (stress exponent $n>1)$ or linear $(n=1)$. In case of linear rheology, a depth-dependent decreasing function is assumed for the Newtonian viscosity

$$
\eta=\frac{1}{2\left(A_{0}^{*}+A_{1}^{*} y\right)}
$$

where $A_{0}^{*}$ and $A_{1}^{*}$ are constants. This expression is extended to the effective viscosity in nonlinear rheology, and a computer program has been written to implement both linear and nonlinear rheologies in a uniform format. 
Case A (Figure 5.6) serves as a reference case with constant Newtonian viscosity $\left(\eta=10^{22} \mathrm{~Pa} \mathrm{~s}\right)$, and constant basal shear $\operatorname{stress}\left(\tau_{b}=57.44 \mathrm{MPa}\right)$. The top velocity boundary condition is specified such that the $u$ component varies linearly with $x$ (resulting in a constant longitudinal strain rate of $\dot{\varepsilon}_{x x}=-10^{-15} \mathrm{~s}^{-1}$ on the upper surface of the block) while the $v$ component is constant. These velocity boundary conditions are compatible with Nye's one-dimensional solution (Nye, 1957). Consequently, computational results for case A yield simply a two-dimensional representation of Nye's one-dimensional solution in the case of Newtonian viscosity. (Comparisons between the one-dimensional and twodimensional solutions in the case of nonlinear rheology will be provided by cases $\mathrm{E}$ and F.)

The results for case A are presented in Figure 5.6, showing (a) the geometry of the originally-rectangular block after $1 \mathrm{Ma}$ of deformation; (b) the velocity field within the block; (c) the maximum shear stress trajectories $\left(\tau_{\max }=\left(\sigma_{1}-\sigma_{2}\right) / 2\right)$, which coincide with potential faulting surfaces in a frictionless (plastic) material; (d) the depth distribution of the "nongravitational" normal and shear stress components (i.e., the functions $F(y)$ and $G(y)$ ) along the rear vertical edge of the block (which in this case are constant; note that a tectonic compression is required to maintain the deformation regime); (e) contours of effective strain rate; and (f) contours of effective shear stress. Both effective strain rate and effective shear stress in this case vary with depth only. Also, the longitudinal normal deviatoric stress $\sigma_{x x}^{\prime}$ $(=F(y) / 2)$ does not vary with $x$ and is negative at both the front and the back edges of the block, resulting in a state of longitudinal compression.

Results for case B are shown in Figure 5.7. Comparison between cases A and B shows the effect of a depth-dependent Newtonian viscosity on the stress, strain rate and velocity fields. The viscosity in case B decreases from $10^{22} \mathrm{~Pa} \mathrm{~s}$ on the top to $10^{21} \mathrm{~Pa} \mathrm{~s}$ on the bottom of the block (see Table 5.3); consequently the material becomes softer with increasing depth, increasing the relative deformation between the lower and upper parts of the block (Figure 5.7a; note that the scaling factors used in plotting results for the various 
magnification factor $=2.1 \mathrm{e} 0$
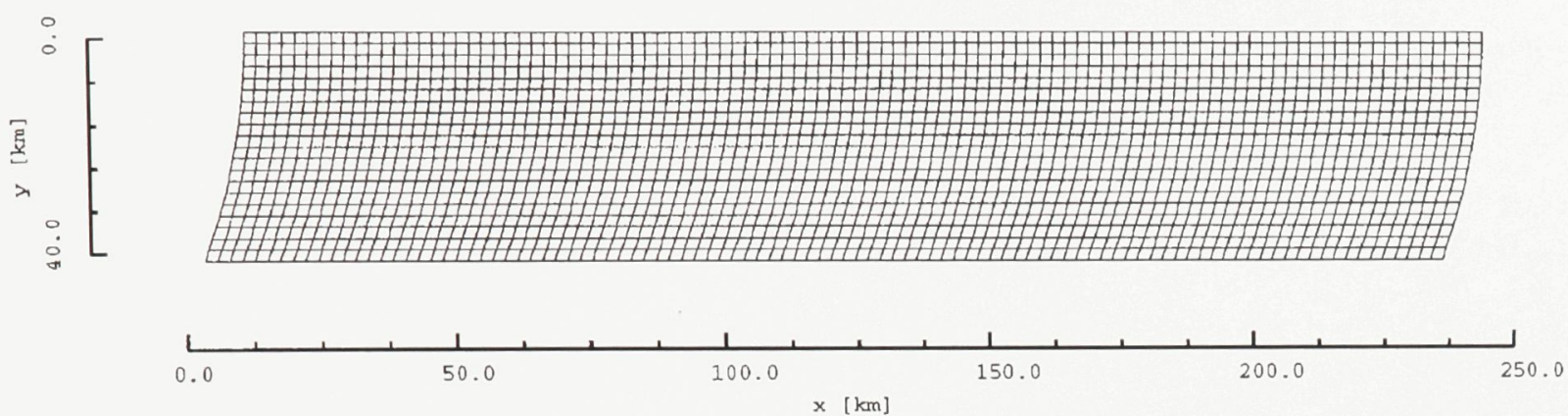

velocity field

(b)

$\max$ vel vector $[\mathrm{mm} / \mathrm{a}]=\stackrel{1.000 \mathrm{e} 2}{\longrightarrow}$
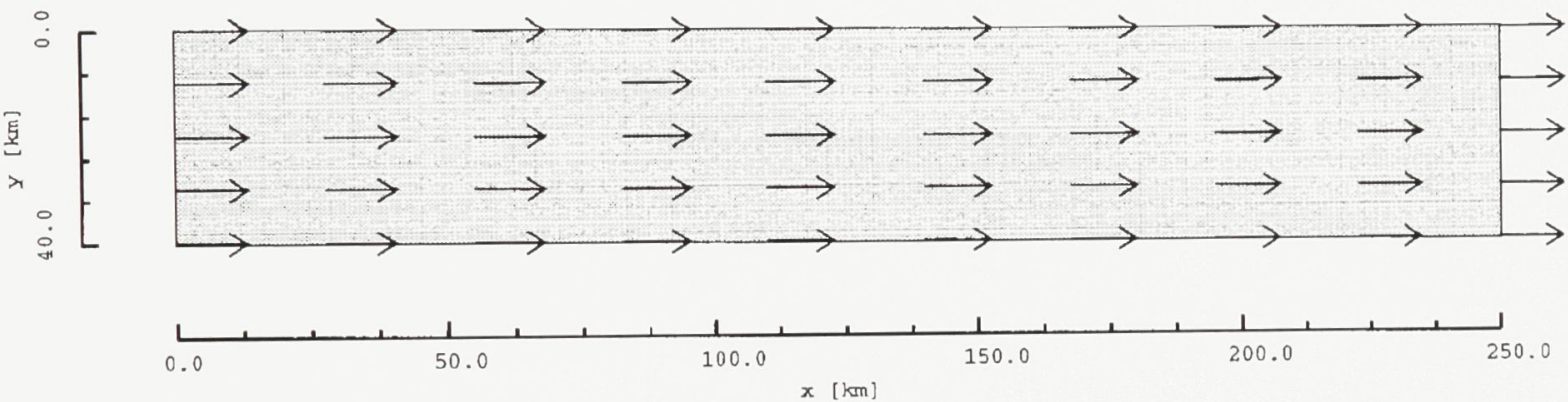

Potential Faulting Trajectories

(c)

Internal Friction Angle [deg] $=45$

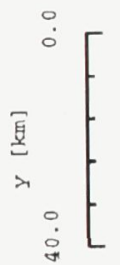
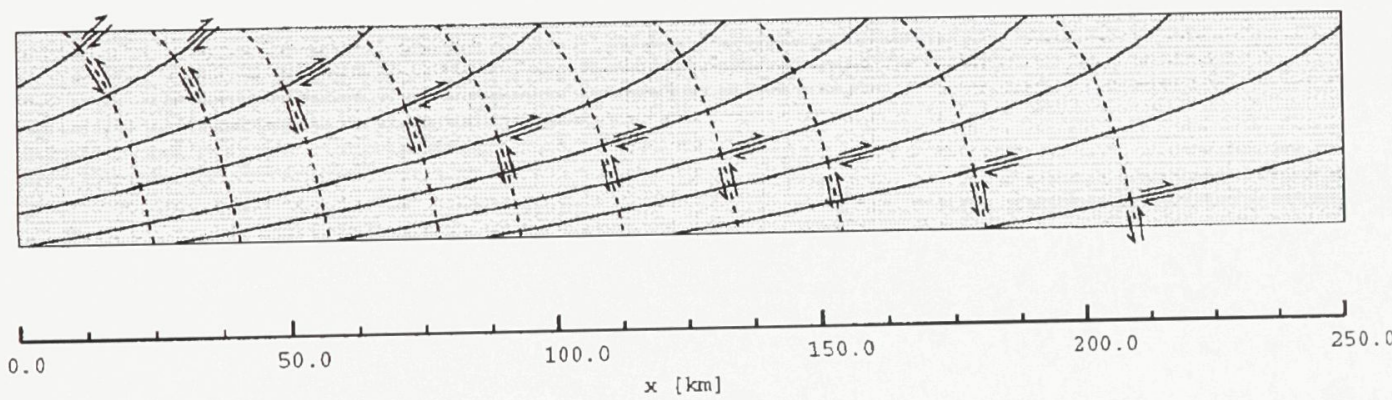

Figure 5.6 Stress and deformation within the block for case A (Table 5.3). (a) deformed shape of the block after $1 \mathrm{Ma}$; (b) velocity field; (c) maximum shear stress trajectories and type of potential faults; 
Tectonic Stress Components at Rear [MPa]

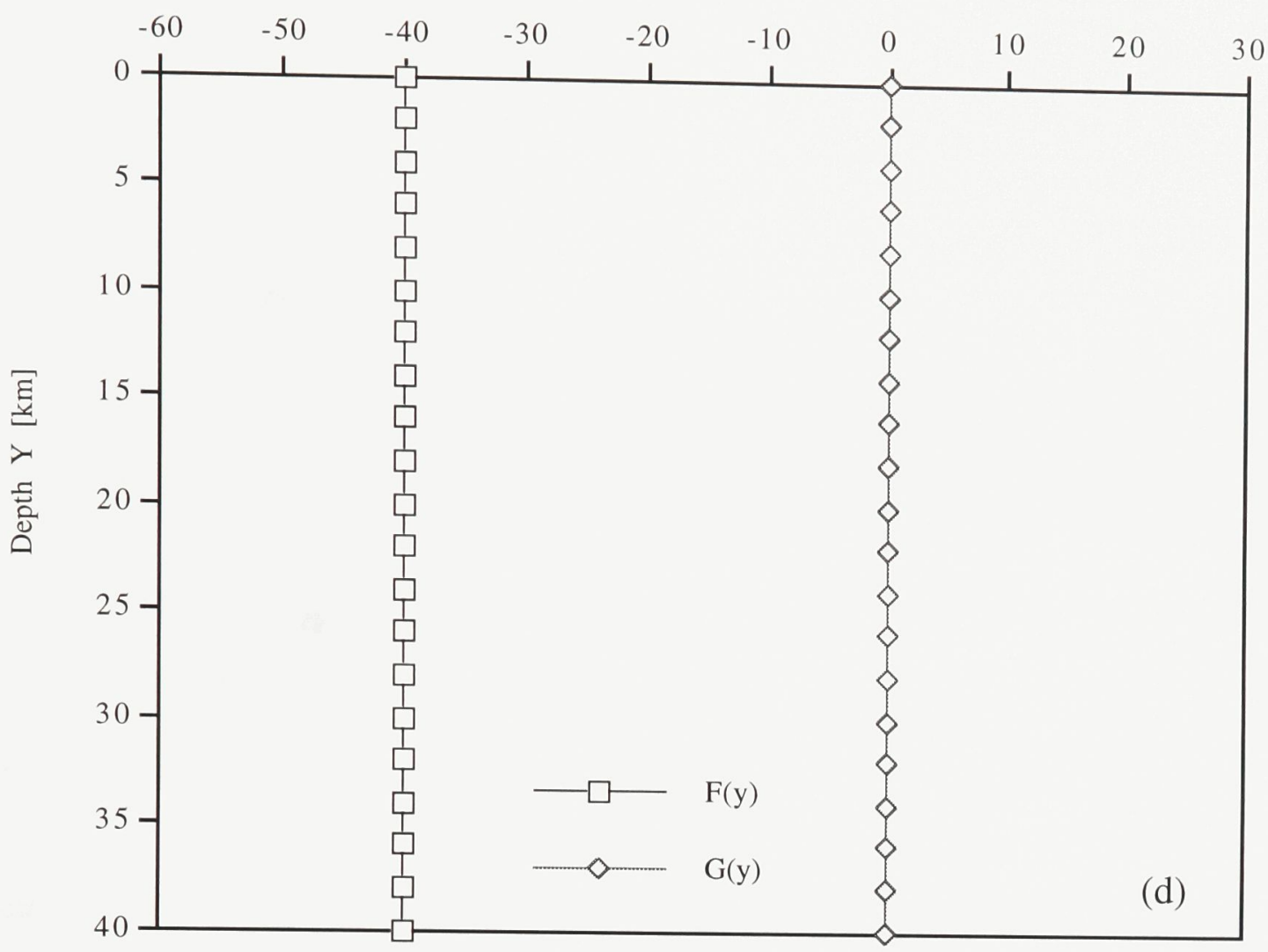

second invar. strain rate, [1/s]

(e)

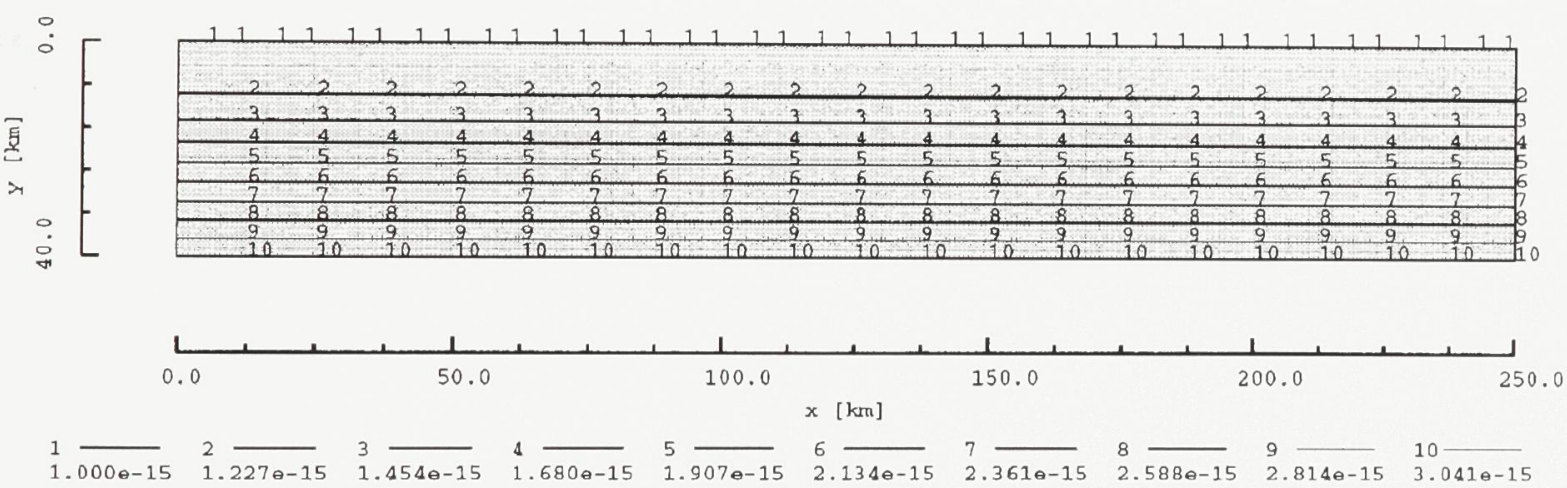

Figure 5.6 (d) tectonic-induced normal and shear stresses along the rear of the block; (e) second invariant of strain rate contour map; (continued) 


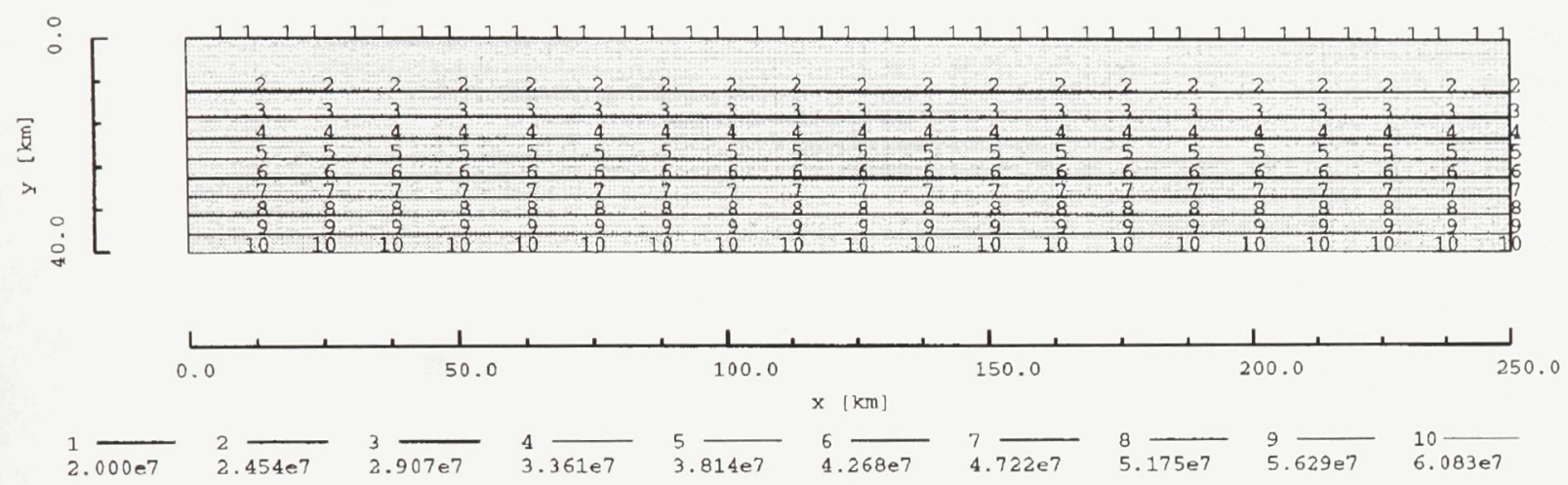

Figure 5.6 (f) second invariant of deviatoric stress contour map. 


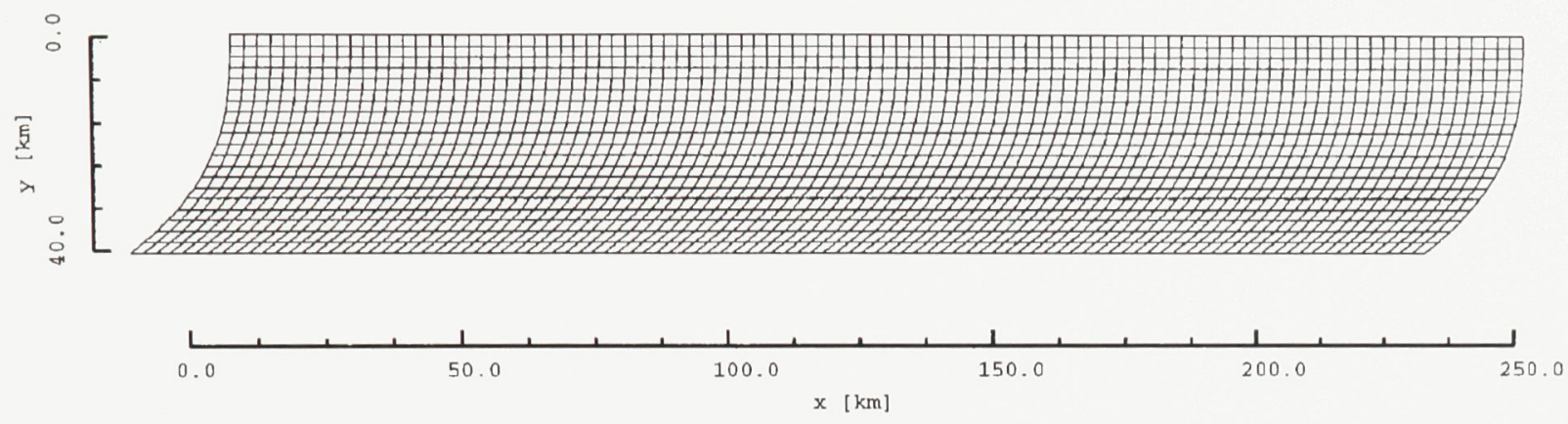

velocity field

(b)

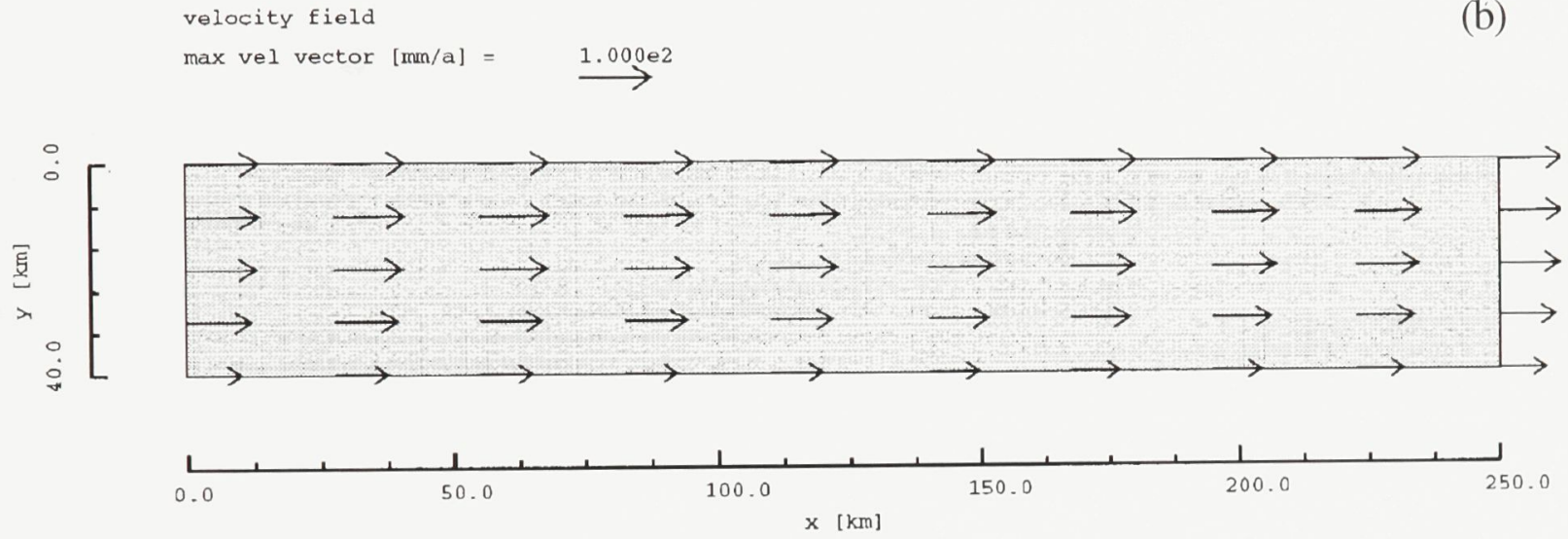

Potential Faulting Trajectories

(c)

Internal Friction Angle [deg] $=45$

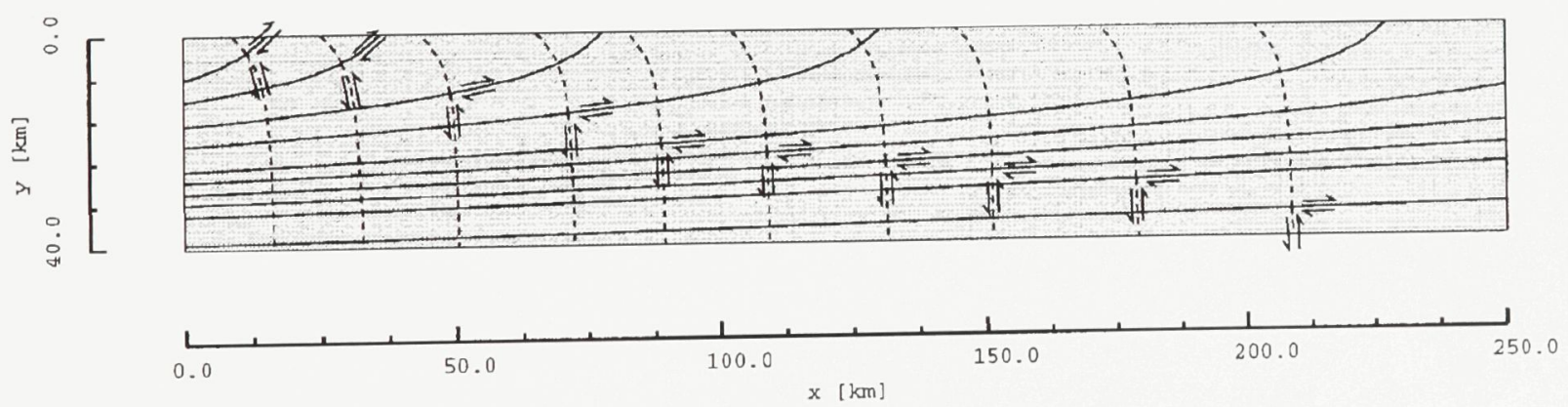

Figure 5.7 Stress and deformation within the block for case B (Table 5.3). (a) deformed shape of the block after $1 \mathrm{Ma}$; (b) velocity field; (c) maximum shear stress trajectories and type of potential faults;

(continued) 
Tectonic Stress Components at Rear [MPa]

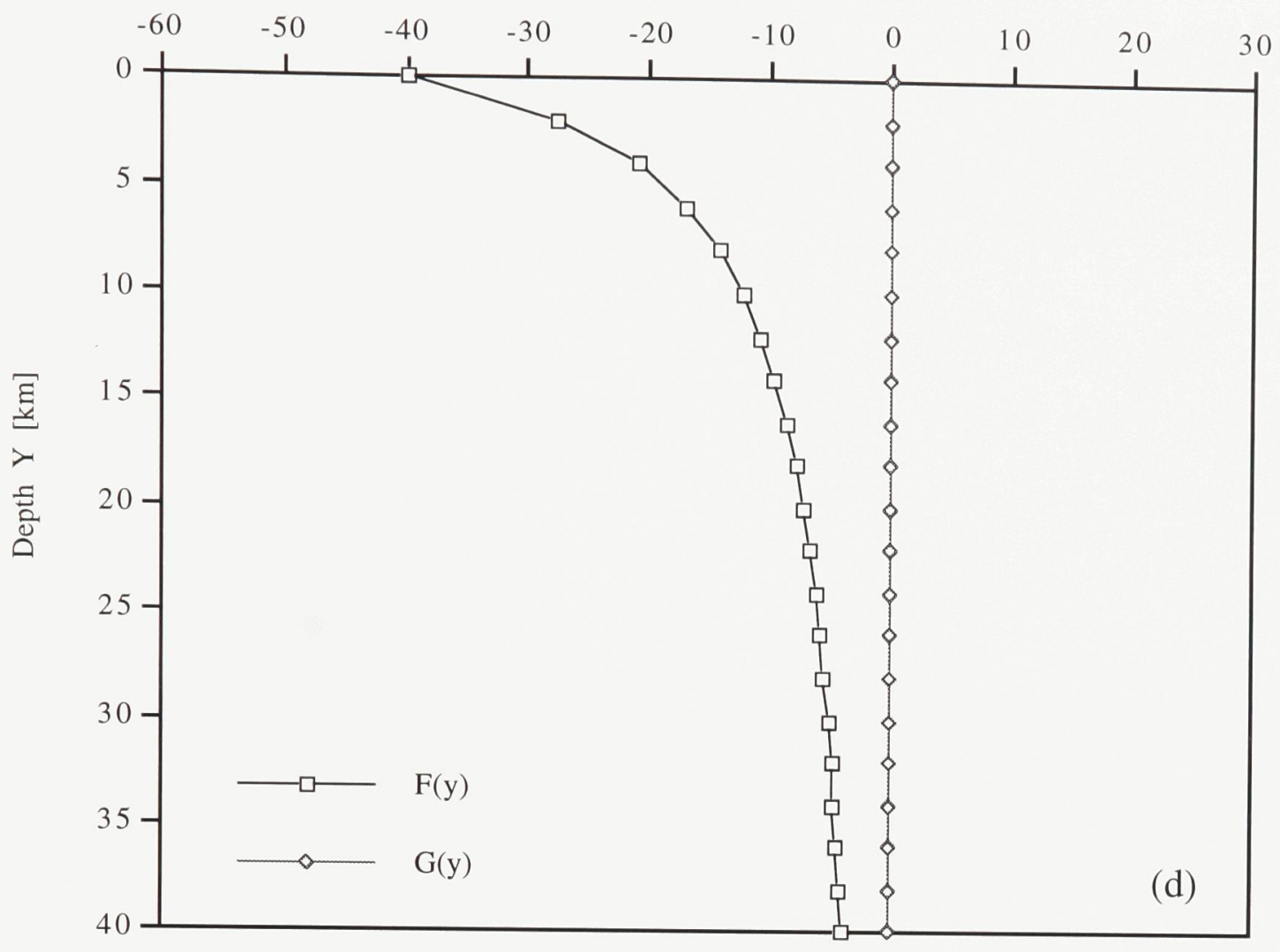

second invar, strain rate, [1/s]

(e)

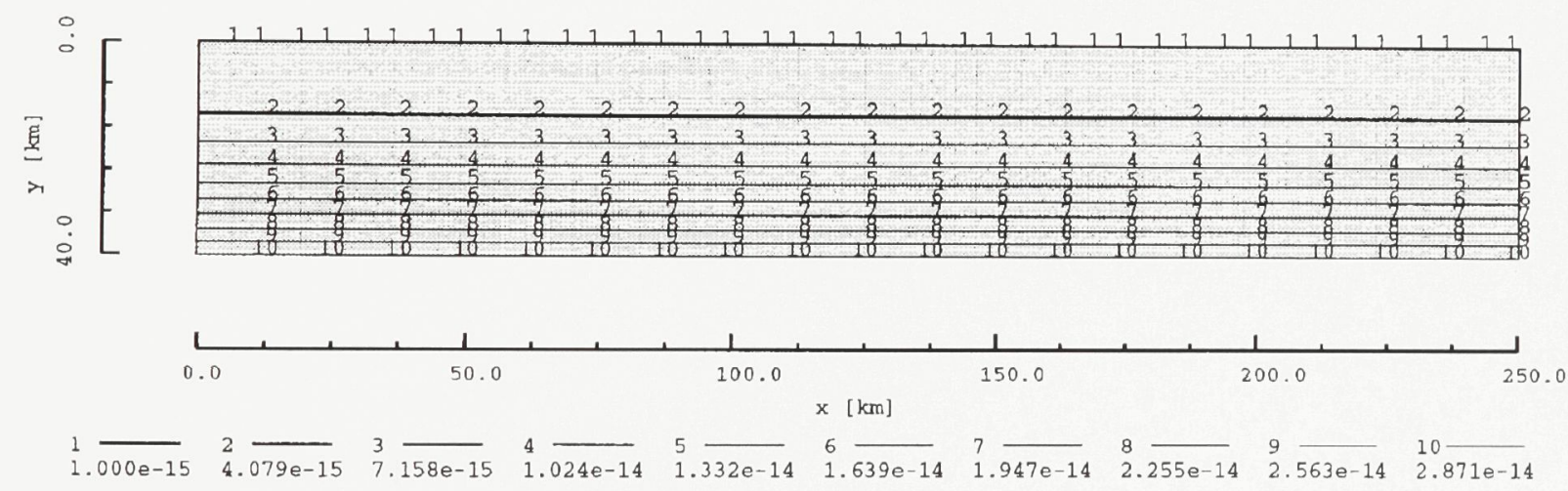

Figure 5.7 (d) tectonic-induced normal and shear stresses along the rear of the block; (e) second invariant of strain rate contour map; (continued) 

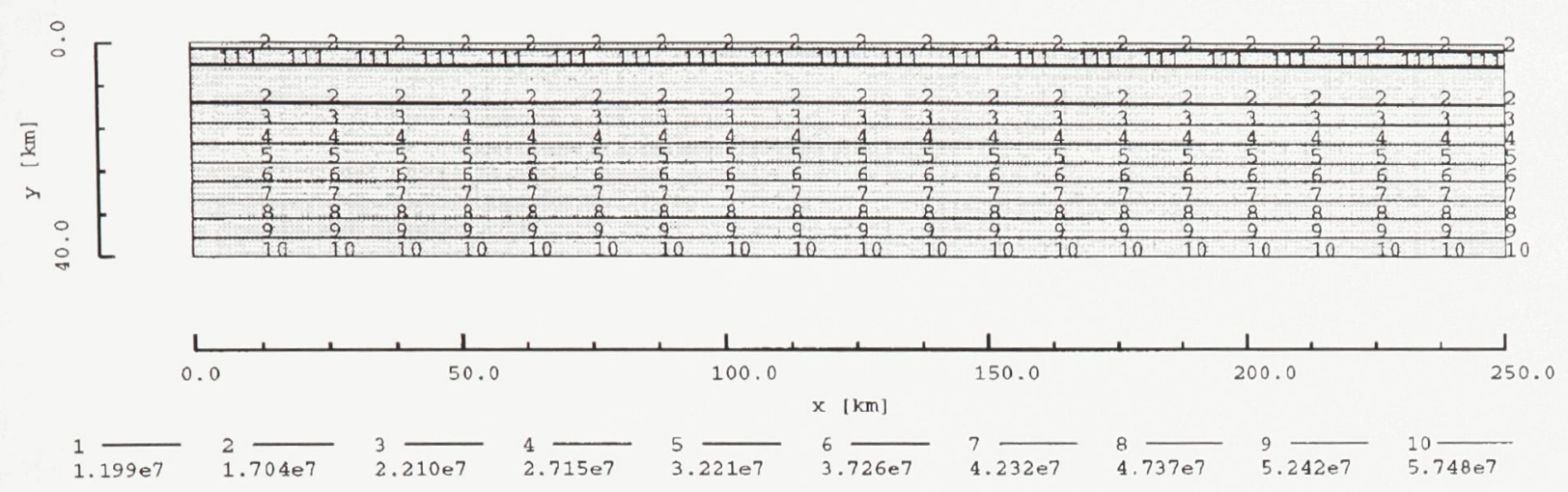

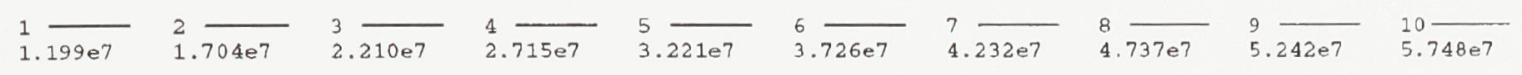

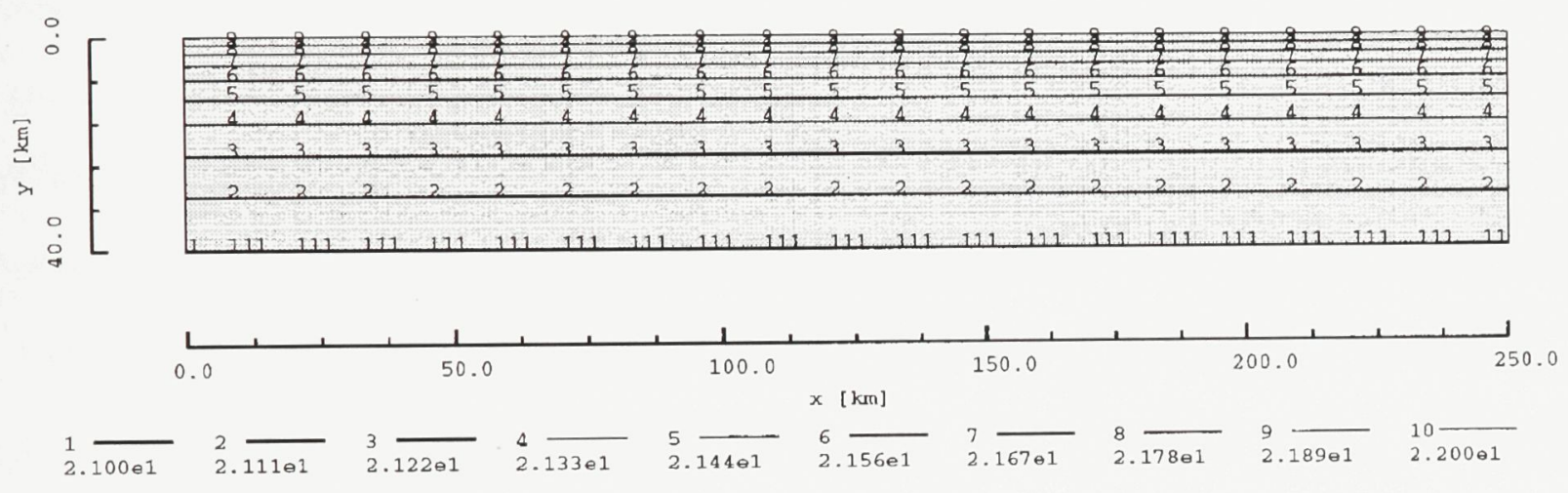

Figure $5.7 \quad$ (f) second invariant of deviatoric stress contour map; (g) Newtonian viscosity contour map (logarithm to base 10) 
cases are not the same). This is reflected also in the velocity field (Figure 5.7b), which shows a marked decrease with increasing depth. The maximum shear stress trajectories (Figure 5.7c) tend to become parallel to the base of the block with increasing depth, indicating an increasingly laminar flow. The nongravitational normal stress at the rear of the block necessary to maintain this deformation regime (Figure 5.7d) decreases in magnitude from $-40 \mathrm{MPa}$ on the top to $-4 \mathrm{MPa}$ at the bottom of the block. The spatial distribution of the effective shear strain rate $\dot{\varepsilon}_{E}^{\prime}$ (Figure 5.7e) shows that the magnitude of deformation in the lower part of the block in case B is approximately one order larger than that in case A. The effective shear stress $\sigma_{E}^{\prime}$ (Figure 5.7f) in the lower part of the block, however, is roughly the same as that in case A, reflecting the fact that same basal shear stress boundary condition is assigned in both cases A and B. Note that, for incompressible material, the effective shear stress $\sigma_{E}^{\prime}$ is the same as the maximum shear stress $\tau_{\max }=\left(s_{1}-s_{2}\right) / 2$. It is also noted from Figure 5.7f that $\sigma_{E}^{\prime}$ first decreases towards a minimum (at a depth of about $5 \mathrm{~km})$ and then increases with depth. Because in this case $\sigma_{E}^{\prime}\left(=\left[F(y)^{2} / 4+\left(\bar{\rho} g_{x} y\right)^{2}\right]^{1 / 2}\right)$ is a function of $F(y)$ and the gravitational shear stress, such a distribution of $\sigma_{E}^{\prime}$ as shown in Figure $5.7 \mathrm{f}$ is a direct consequence of the specified depth distribution of the Newtonian viscosity which largely determines the function $F(y)$ (see Figure 5.7d). The spatial variation of the Newtonian viscosity of the block is shown in Figure $5.7 \mathrm{~g}$. Similar to case A, normal deviatoric stresses are negative at both the front and the back edges of the block.

Case $\mathrm{C}$ (Figure 5.8) differs from case $\mathrm{A}$ in two respects: (1) the $u$ component of the velocity boundary condition on the top surface results in a longitudinal strain rate varying linearly in the $x$-direction (decreasing shortening from the front towards the rear of the block); and (2) the magnitude of basal shear stress is assumed to decrease from the rear toward the front (from 57.44 MPa to 40.44 MPa). The deformed configuration and the velocity field are shown in Figures 5.8a and b, respectively. Both are quite different from those of case A. The velocity decreases from the rear towards the front much faster in case $\mathrm{C}$ than in case A. Also the maximum shear stress trajectories (Figure 5.8c) dip more steeply 
magnification factor $=3.2 \mathrm{e}-1$
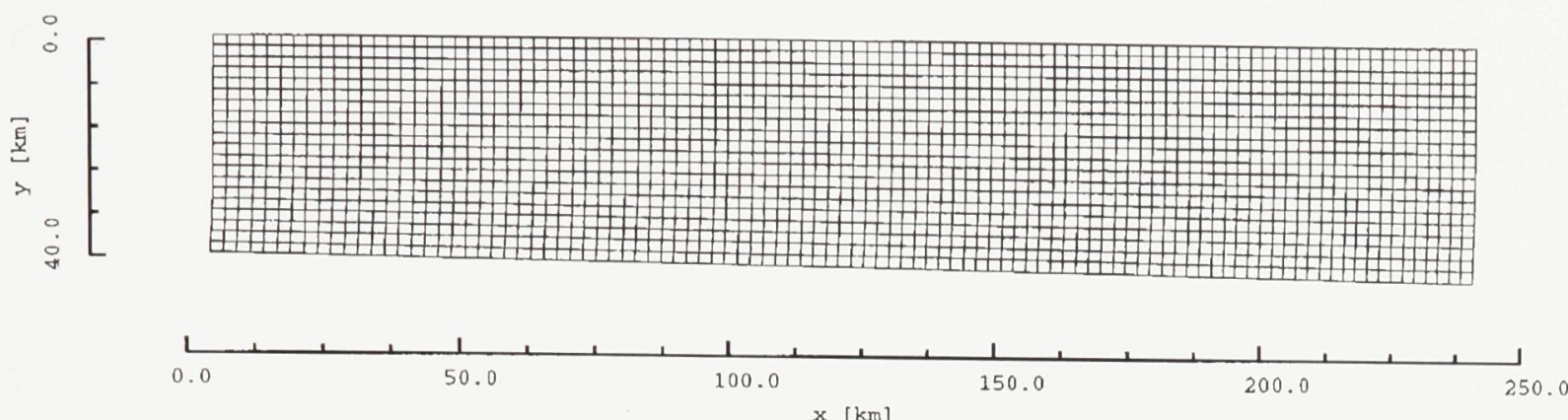

velocity field

(b)

$\max$ vel vector $[\mathrm{mm} / \mathrm{a}]=\stackrel{1.000 \mathrm{e} 2}{\longrightarrow}$
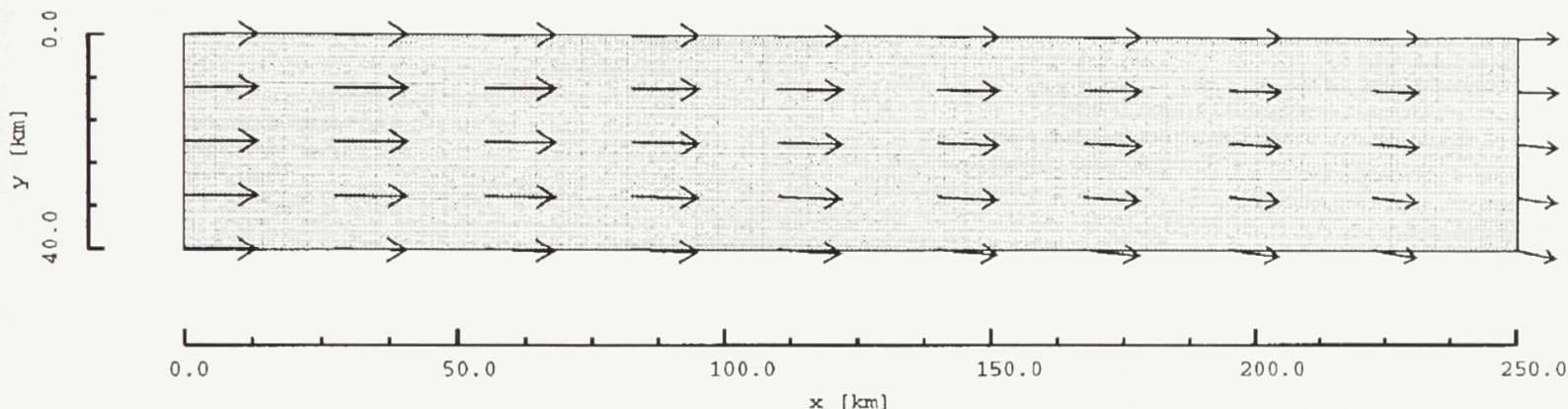

Potential Faulting Trajectories

Internal Friction Angle [deg] $=45$

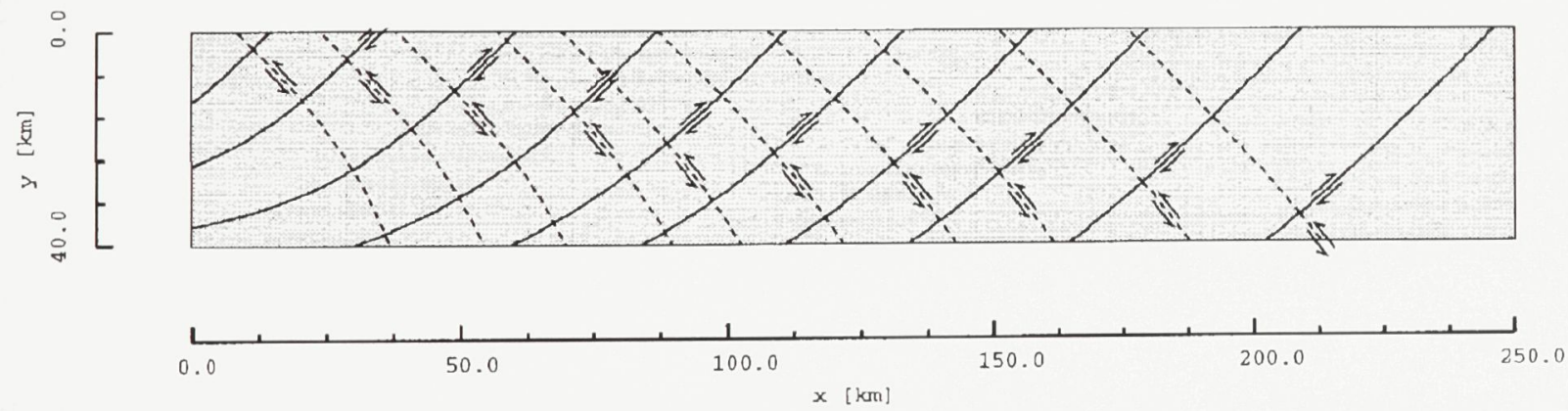

Figure 5.8 Stress and deformation within the block for case C (Table 5.3). (a) deformed shape of the block after $1 \mathrm{Ma}$; (b) velocity field; (c) maximum shear stress trajectories;

(continued) 
Tectonic Stress Components at Rear [MPa]

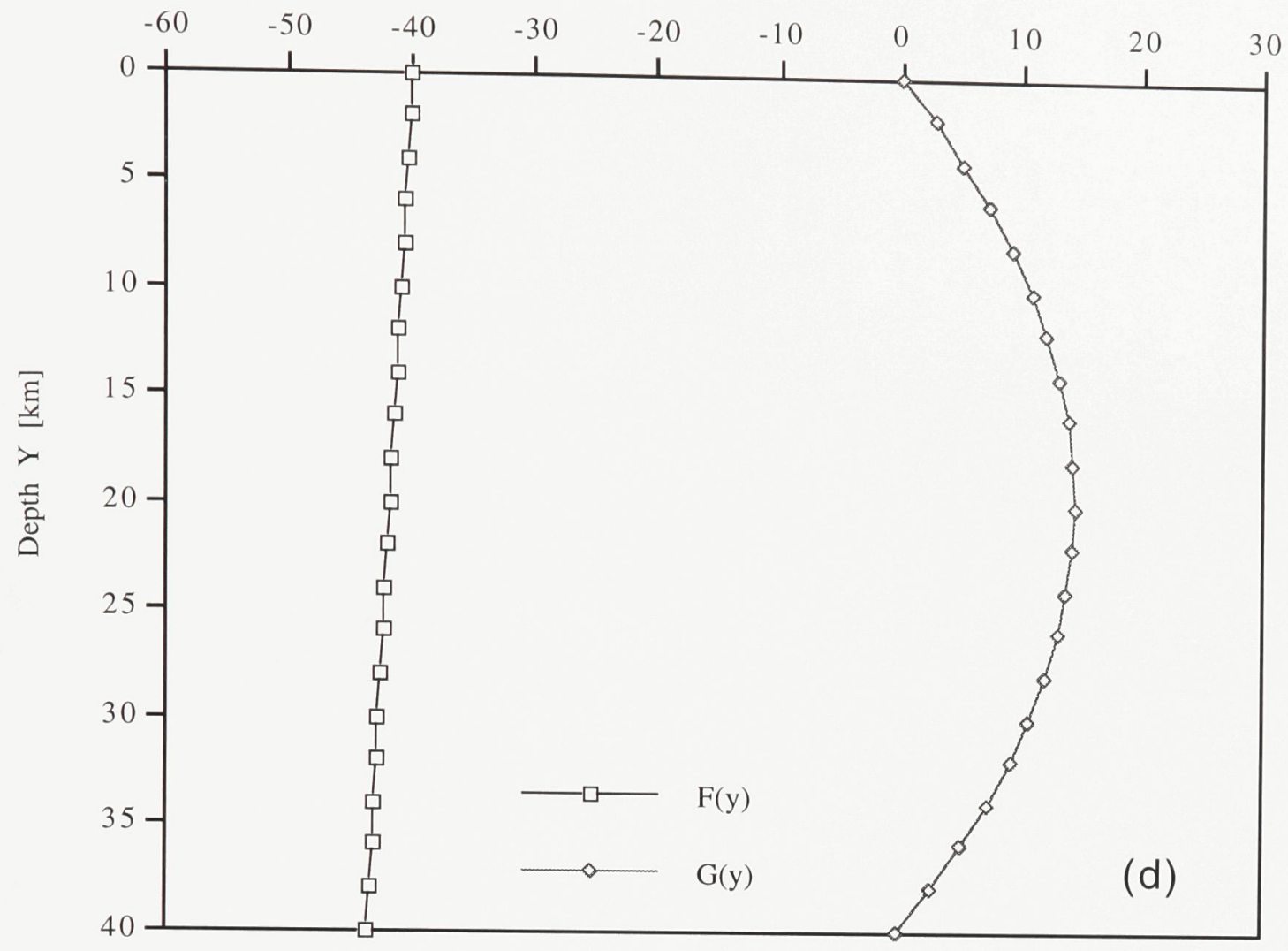

second invar. strain rate, [1/s]

(e)

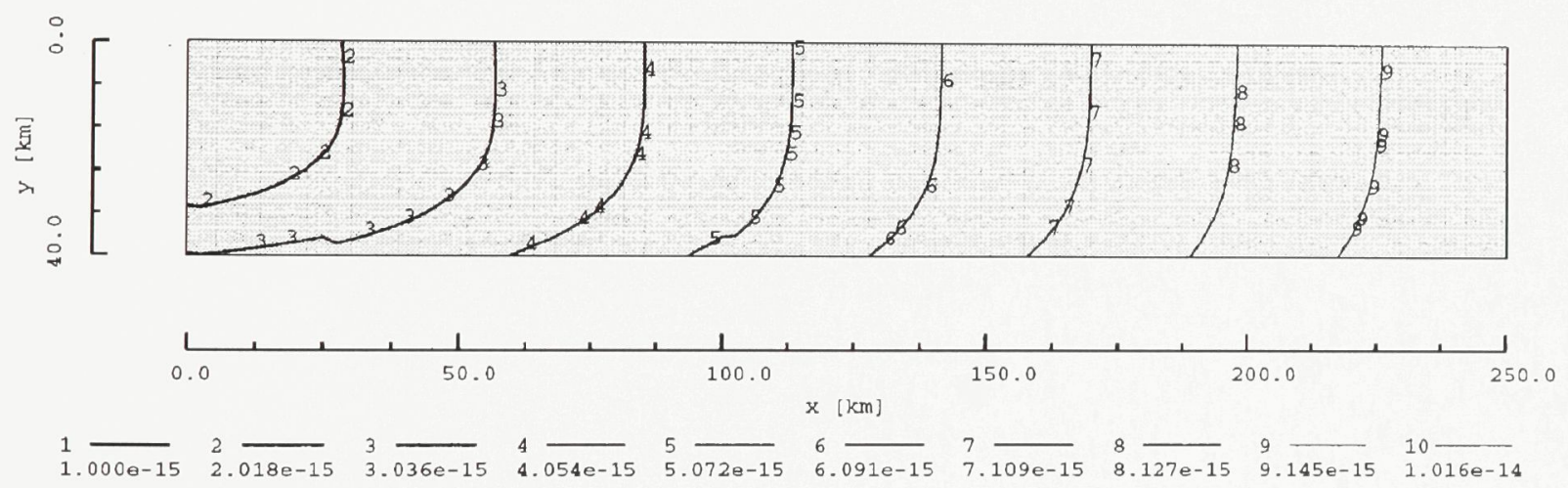

Figure 5.8 (d) tectonic-induced normal and shear stresses along the rear boundary of the block; (e) second invariant of strain rate contour map; (continued) 


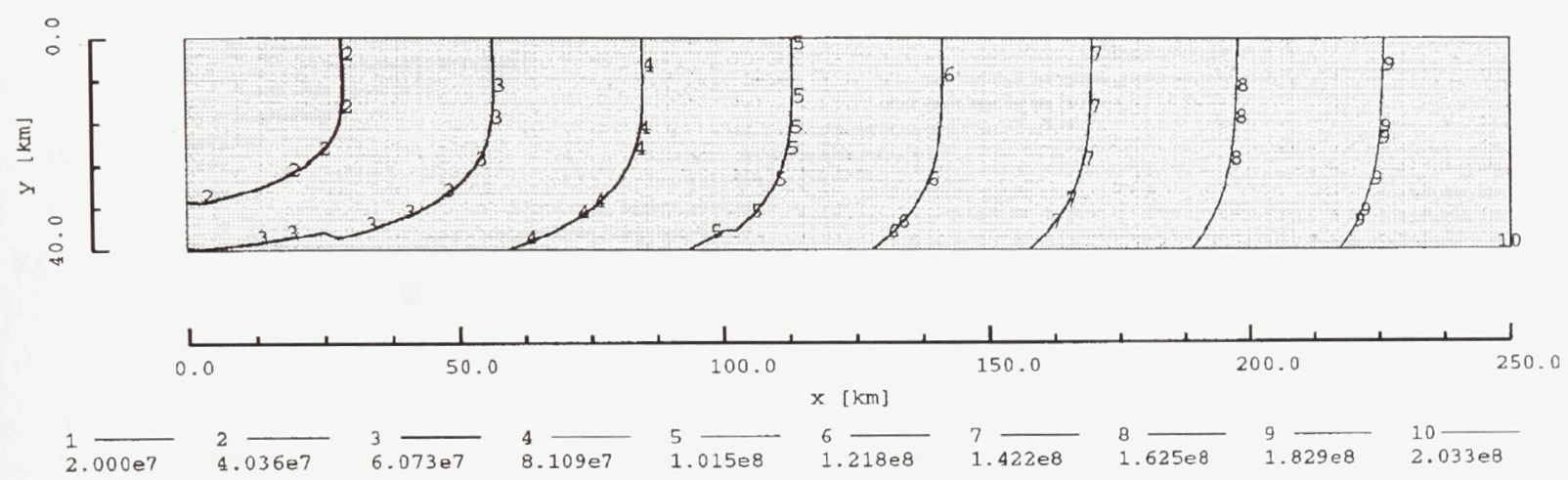

Figure 5.8 (f) second invariant of deviatoric stress contour map. 
in case $\mathrm{C}$ than in case $\mathrm{A}$. The nongravitational normal stress at the rear of the block necessary to maintain this deformation regime (Figure 5.8d) increases slightly with depth while that in case A remains constant. The spatial distribution of the effective shear strain rate $\dot{\varepsilon}_{E}^{\prime}$ (Figure 5.8e) shows that the deformation in the block varies mainly in the $x$ direction in case $\mathrm{C}$ rather than in the $y$-direction as in case A. Similar comparisons can also be made for the spatial distribution of the effective shear stress $\sigma_{E}^{\prime}$ (Figure 5.8f).

Case D (Figure 5.9) can be used to asses the effect on the solution of the $v$-component of the velocity boundary condition specified on top of the block. Here, the (upward) $v$ -component along the top surface is assumed to increase in magnitude from $0.126 \mathrm{~cm} \mathrm{a}^{-1}$ at rear to $3.28 \mathrm{~cm} \mathrm{a}^{-1}$ at the front. Figure 5.9 a shows the deformation of the block after 1 Ma. The velocity field is plotted in Figure $5.9 \mathrm{~b}$. The velocity distribution is greatly affected by the kinematic boundary conditions on the top surface. Trajectories of maximum shear stress are shown in Figure 5.9c; the depth distribution of tectonic stresses along the rear vertical plane is plotted in Figure 5.9d. A change of the $v$ component of the top velocity boundary condition has no effect on the state of stress within the block (Figure 5.9f). On the other hand, an identical stress field and identical rheology result in an identical strain rate field, as can be seen by comparing effective shear strain rates in Figures 5.6e and 5.9e.

Cases $\mathrm{E}$ and $\mathrm{F}$ consider nonlinear rheology, using the power-law creep parameters of anorthosite ( $c f$. Ranalli, 1995). Results for case E are shown in Figure 5.10 in the usual way, with the addition of a further diagram showing the variation of effective viscosity with position as in case B. Comparison between Figures 5.6 and 5.10 reveals a great deal of similarity between cases $\mathrm{A}$ and $\mathrm{E}$. This is the result of a near-constant effective viscosity throughout the block in case E (Figure 5.10g); the average value of the effective viscosity is very close to the constant Newtonian viscosity specified in case A. However, the difference between linear rheology and power-law rheology becomes obvious from a comparison of the "tectonic" stress along the rear vertical plane necessary to drive the deformation (Figures 5.6d and 5.10d). The normal stress $F(y)$ in case A has a constant value of -40 
(a)

deformation after time $[\mathrm{Ma}]=1.00 \mathrm{e}$

magnification factor $=1.3 \mathrm{e}-1$
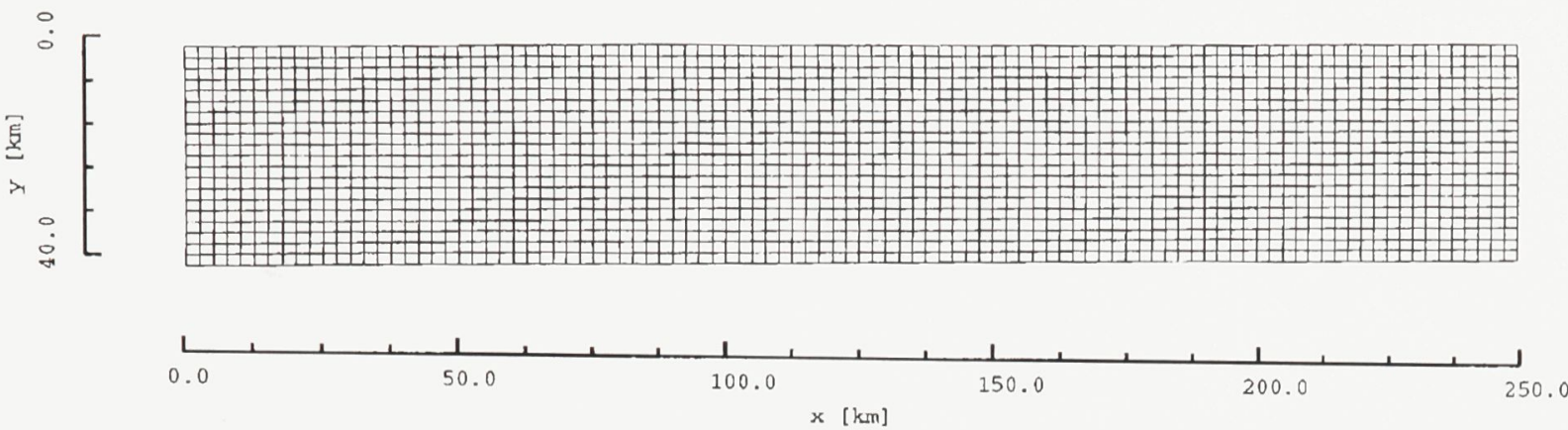

velocity field

(b)
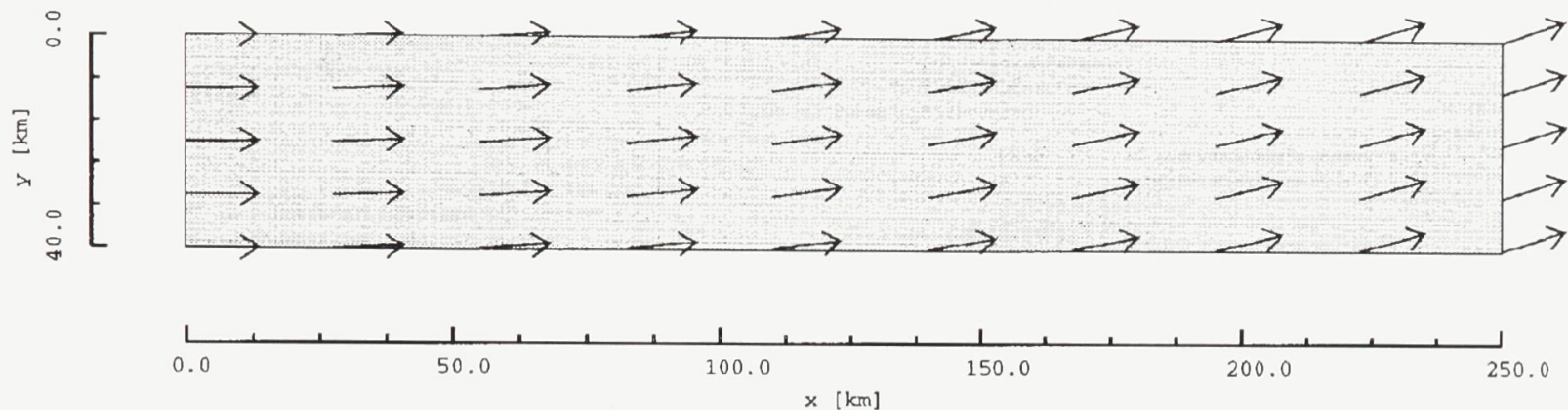

Potential Faulting Trajectories

(c)

Internal Friction Angle [deg] $=45$

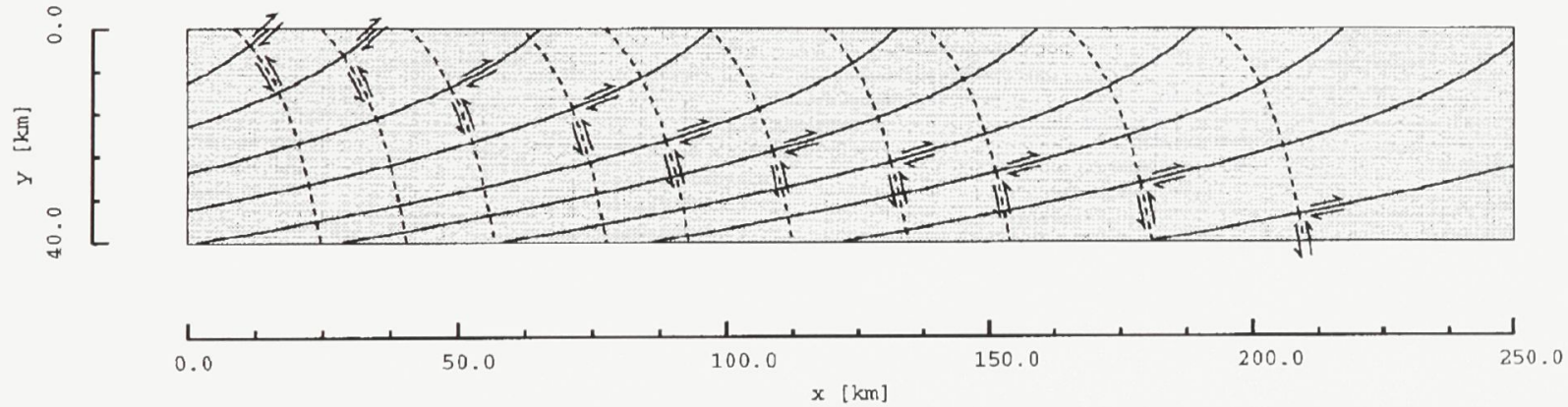

Figure 5.9 Stress and deformation within the block for case D (Table 5.3). (a) deformed shape of the block after $1 \mathrm{Ma}$; (b) velocity field; (c) maximum shear stress trajectories;

(continued) 
Tectonic Stress Components at Rear [MPa]

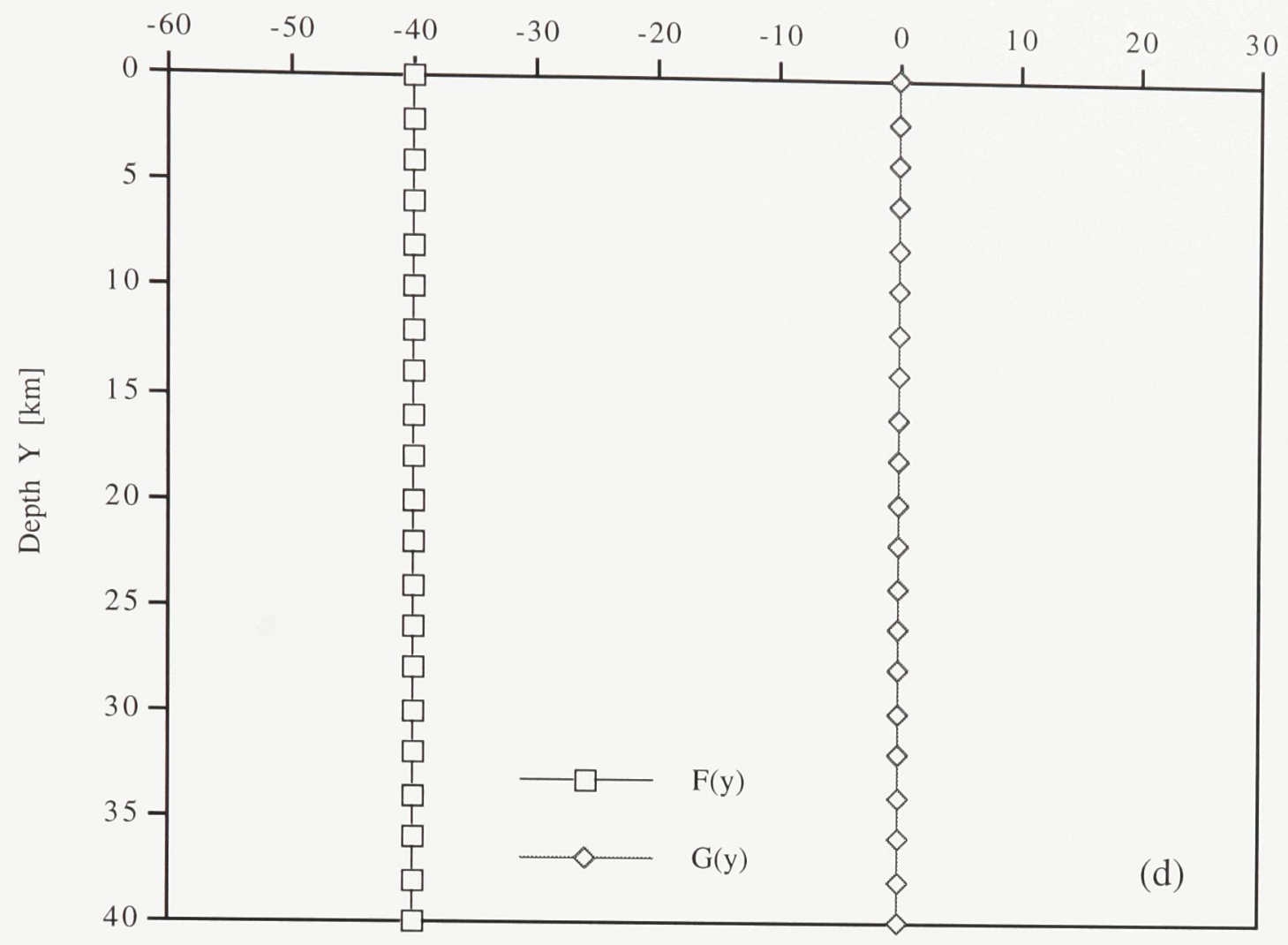

second invar. strain rate, [1/s]

(e)

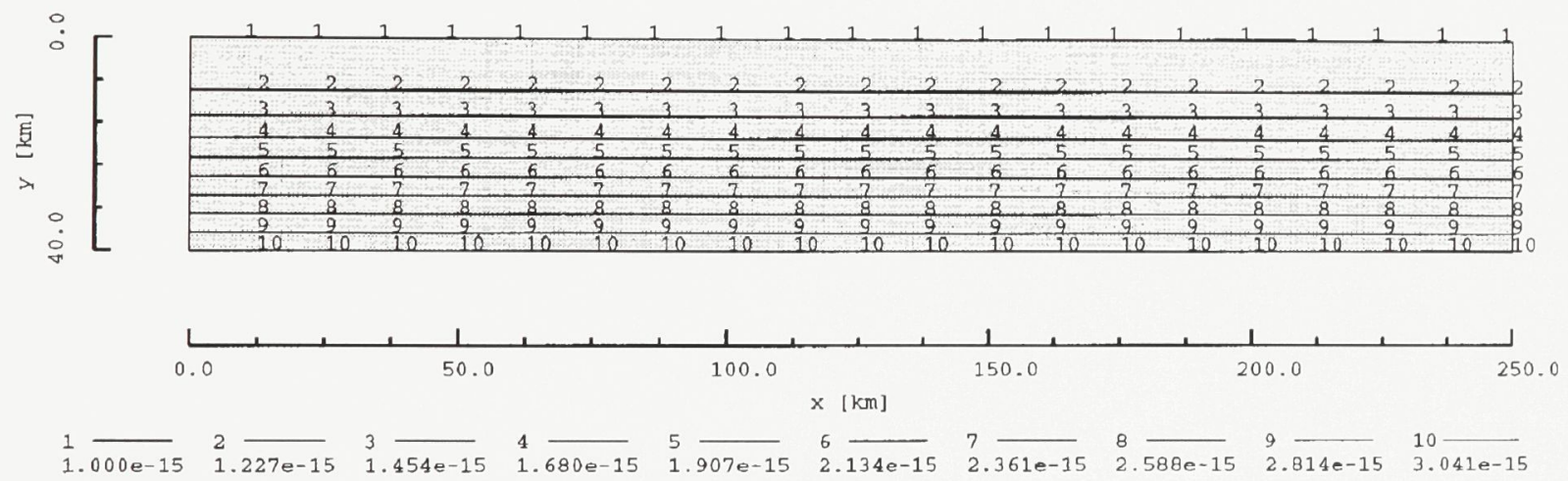

Figure 5.9 (d) tectonic-induced normal and shear stresses along the rear boundary of the block; (e) second invariant of strain rate contour map; (continued) 


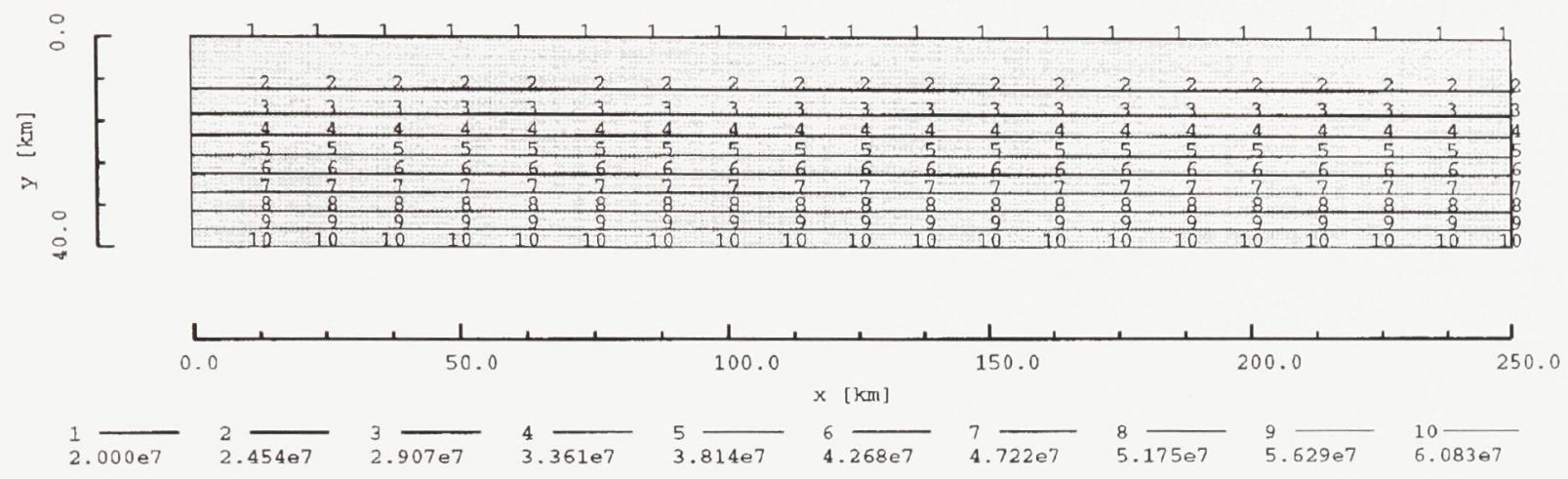

Figure 5.9 (f) second invariant of deviatoric stress contour map. 
deformation after time $[\mathrm{Ma}]=1.00 \mathrm{e} 0$

magnification factor $=2.2 e 0$
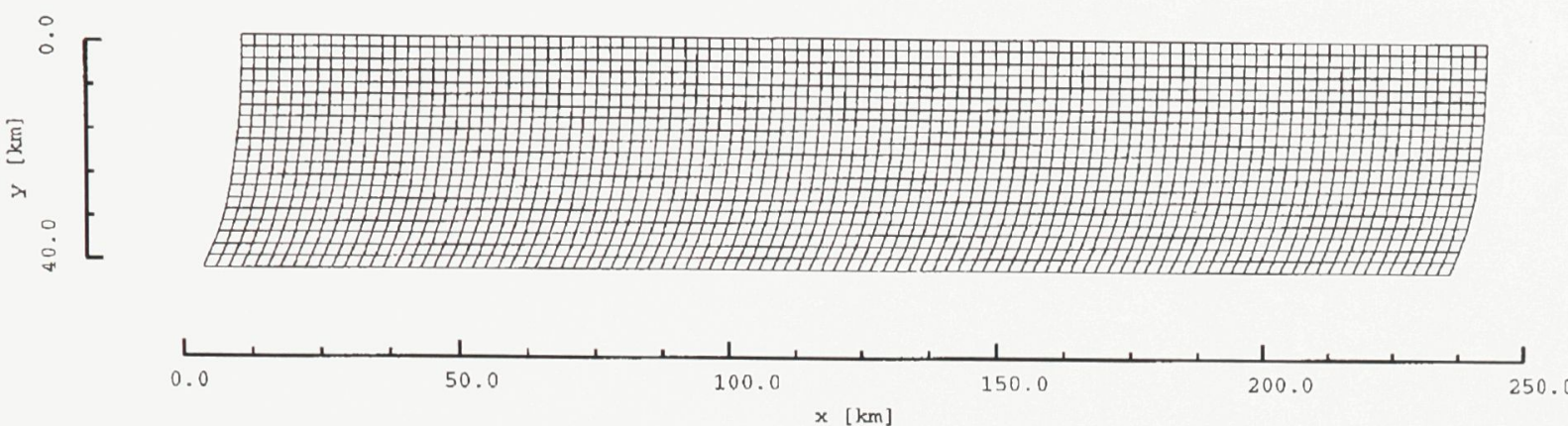

velocity field

$\max$ vel vector $(\mathrm{mm} / \mathrm{a})=\stackrel{1.000 \mathrm{e} 2}{\longrightarrow}$

(b)
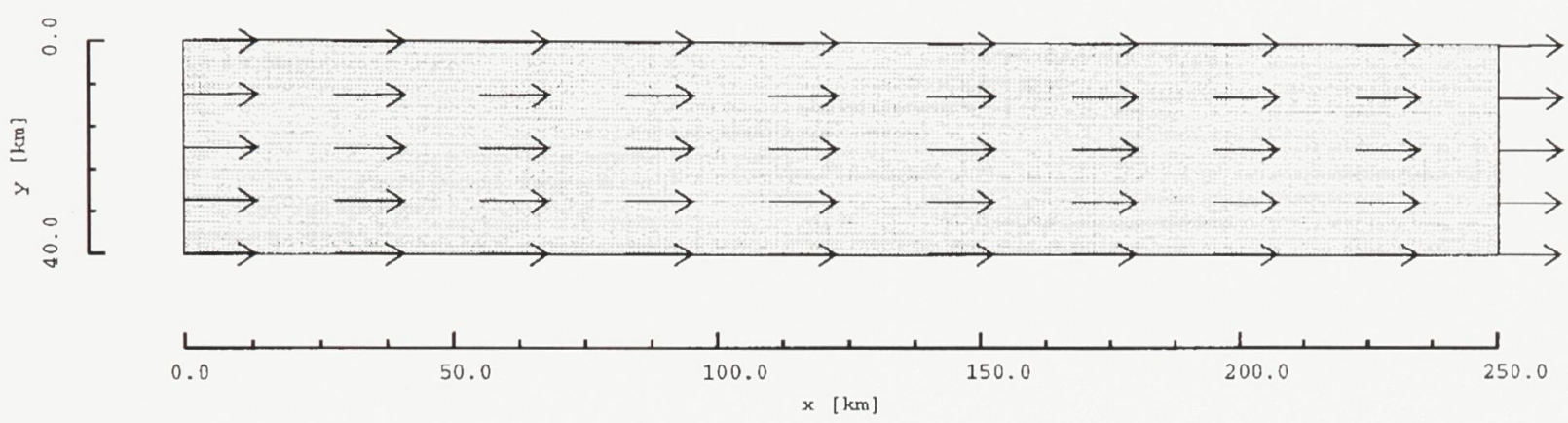

Potential Faulting Trajectories

(c)

Internal Friction Angle [deg] $=45$

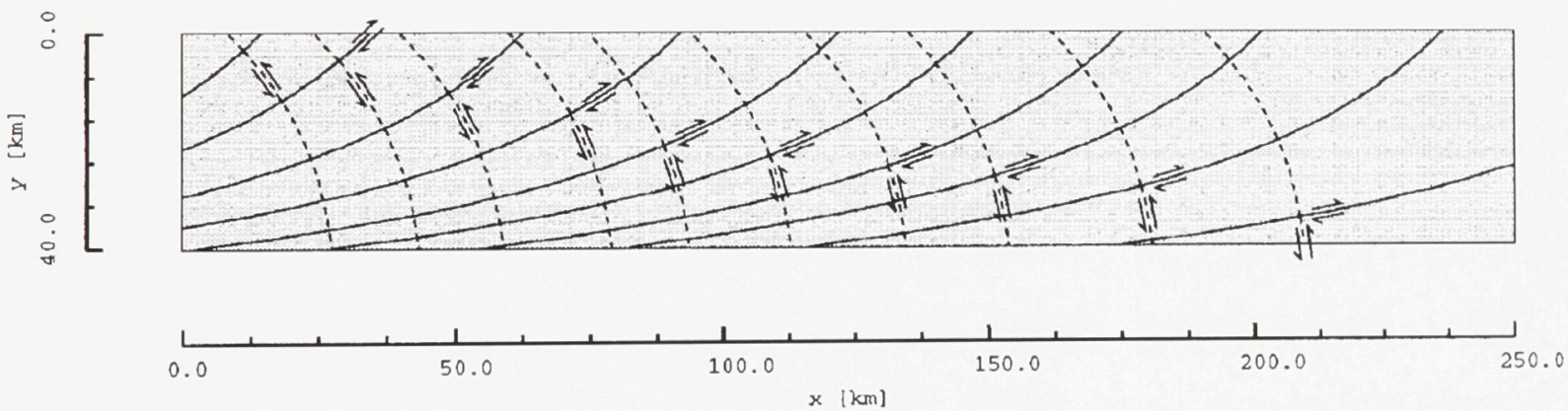

Figure 5.10 Stress and deformation within the block for case E (Table 5.3). (a) deformed shape of the block after $1 \mathrm{Ma}$; (b) velocity field; (c) maximum shear stress trajectories;

(continued) 


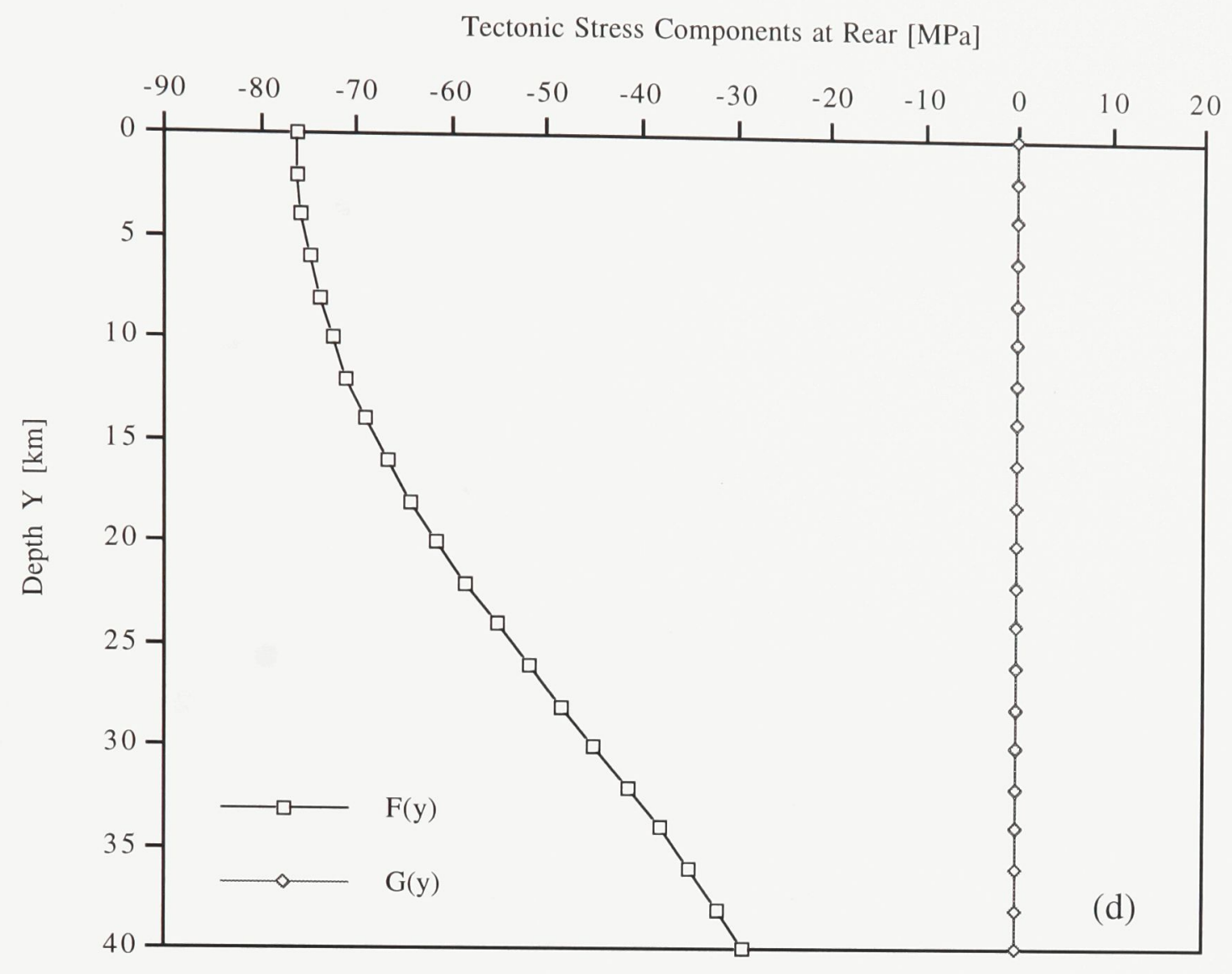

second inver. strain rate, [1/s]

(e)

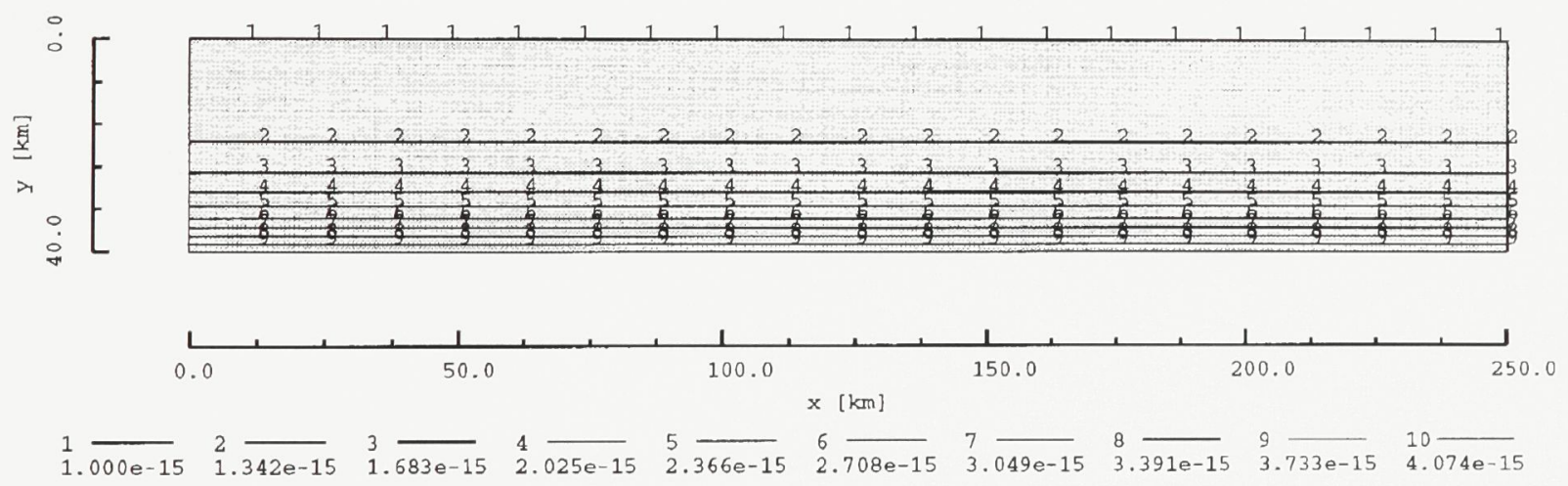

Figure 5.10 (d) tectonic-induced normal and shear stresses along the rear boundary of the block; (e) second invariant of strain rate contour map; (continued) 

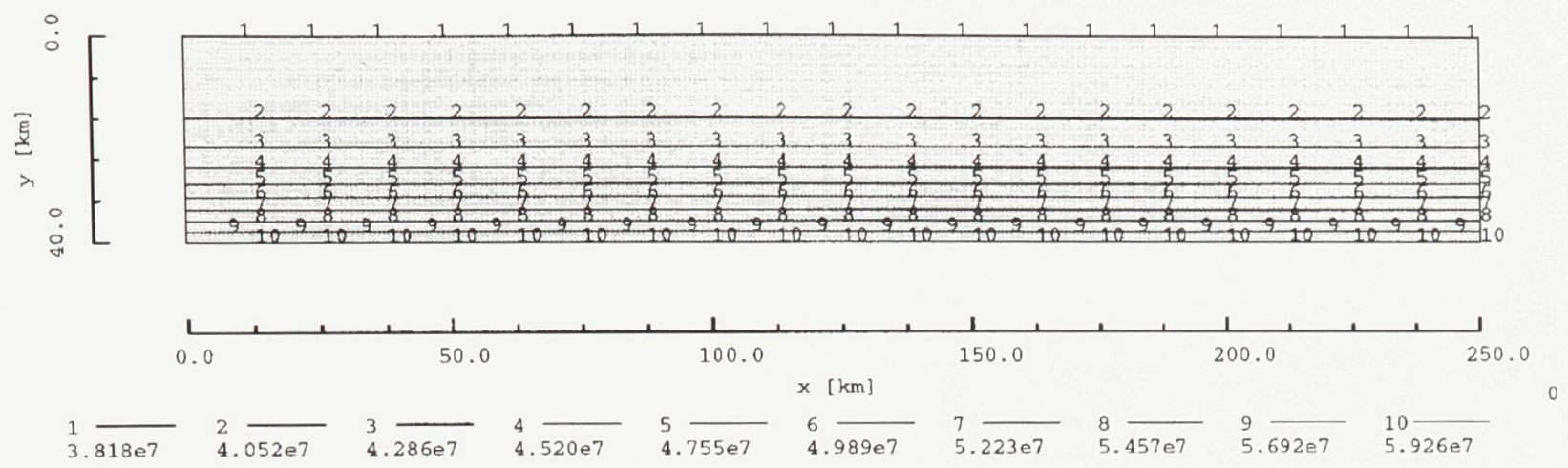

$\log 10[$ eff viscosity] [Pa s]

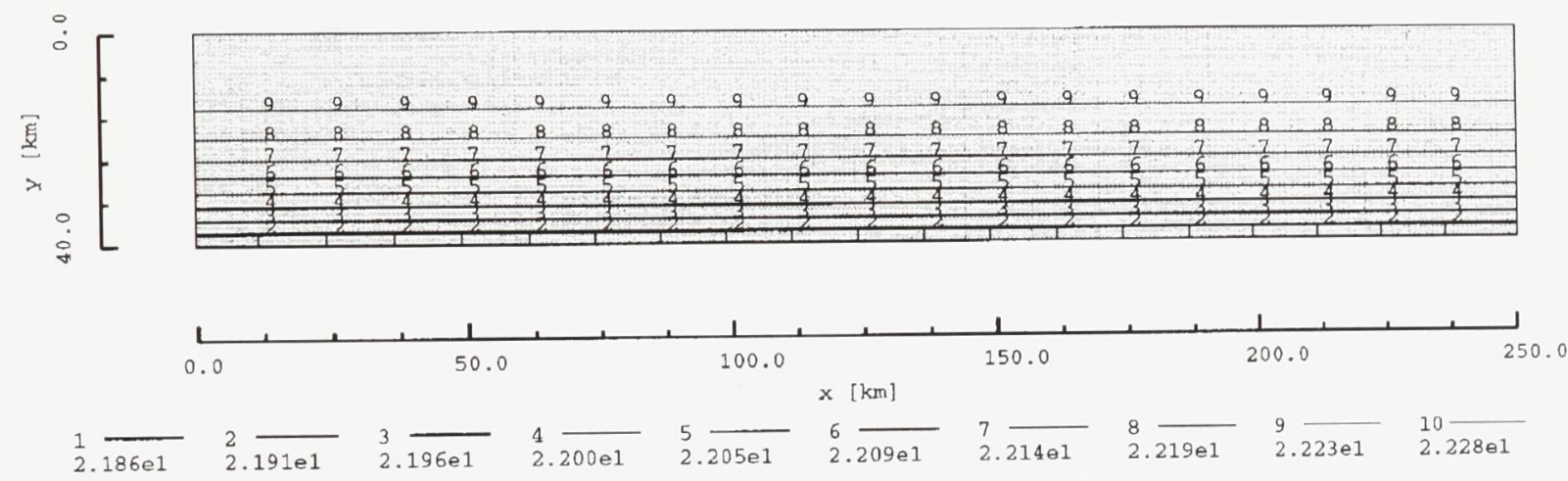

Figure 5.10 (f) second invariant of deviatoric stress contour map; (g) effective viscosity contour map (logarithm to base 10). 
deformation after time $[\mathrm{Ma}]=1.00 \mathrm{e}$

magnification factor $=2.1 \mathrm{e}-1$
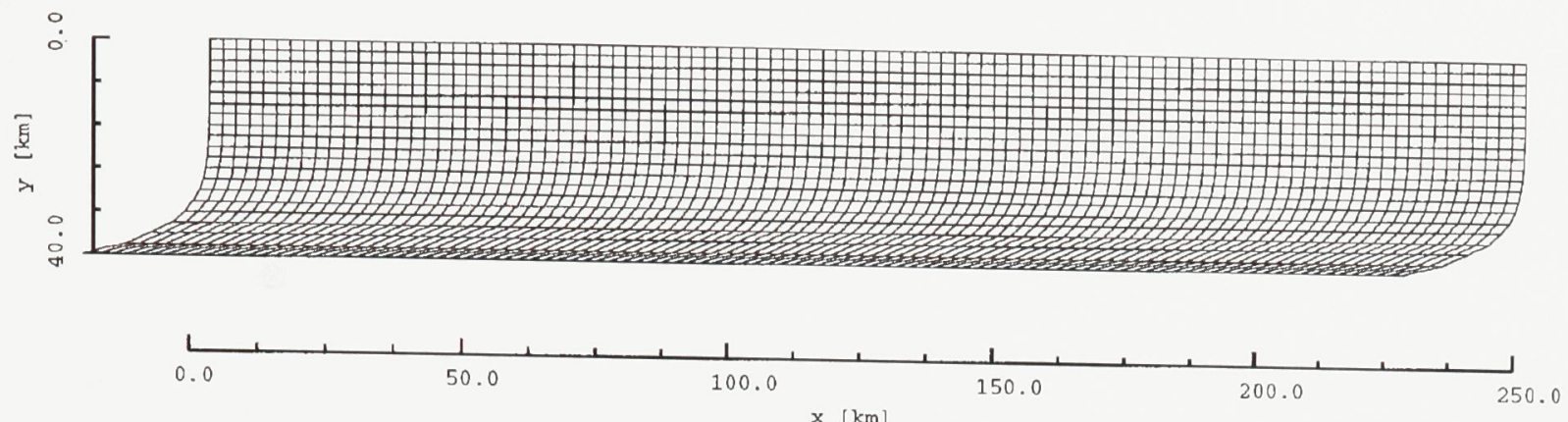

velocity field

$\max$ vel vector $[\mathrm{mm} / \mathrm{a}]=\stackrel{1.000 \mathrm{e} 2}{\longrightarrow}$

(b)
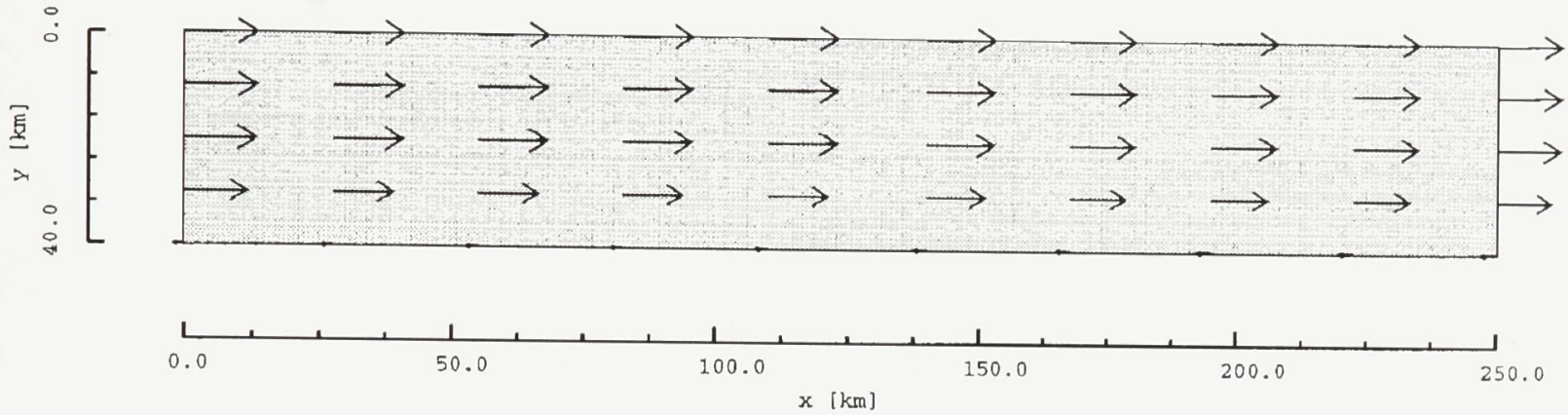

Potential Faulting Trajectories

Internal Friction Angle [deg] $=45$

(c)

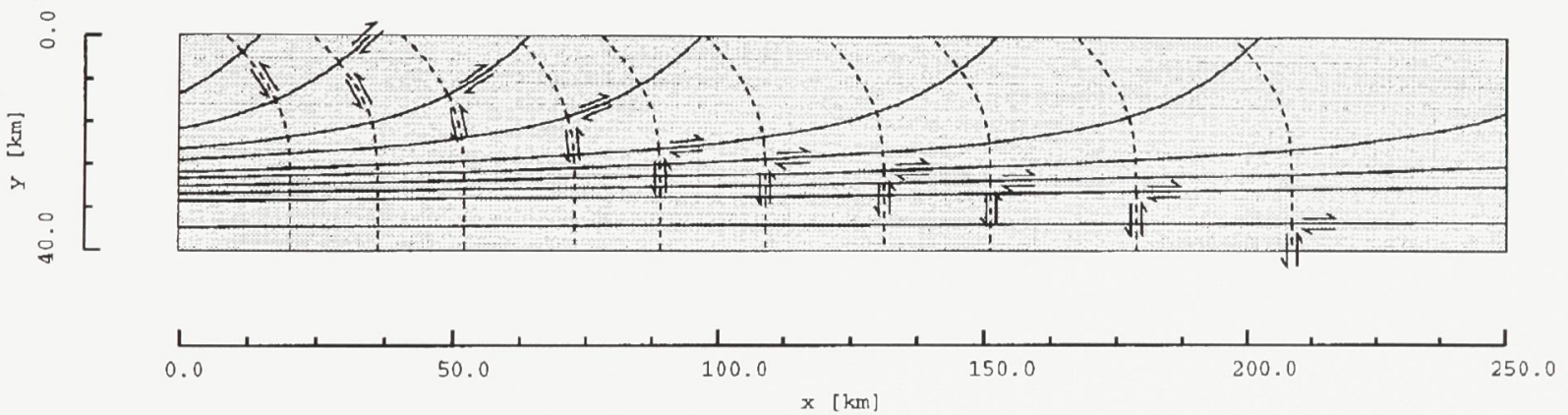

Figure 5.11 Stress and deformation within the block for case F (Table 5.3). (a) deformed shape of the block after $1 \mathrm{Ma}$; (b) velocity field; (c) maximum shear stress trajectories; (continued) 
Tectonic Stress Components at Rear [MPa]

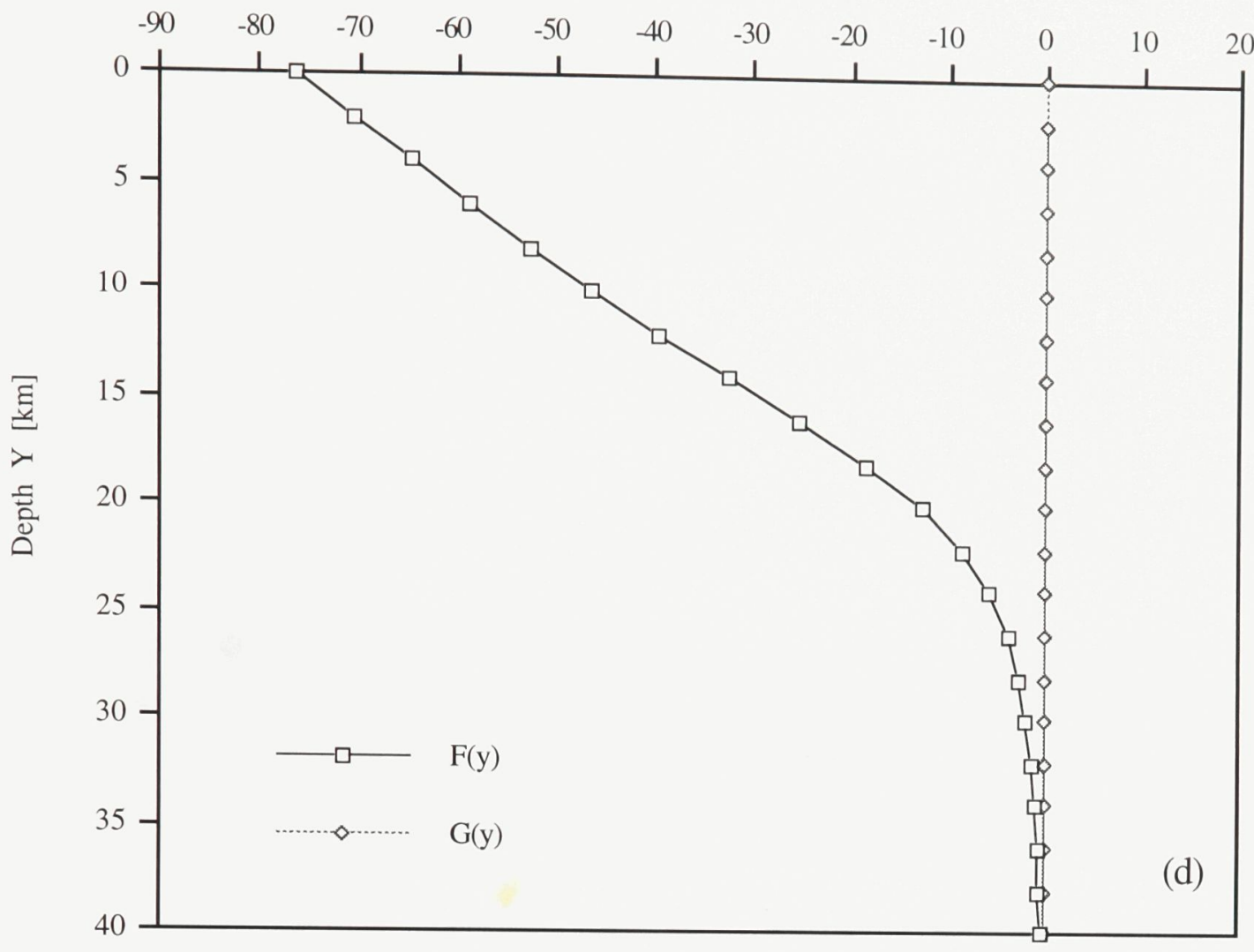

second invar, strain rate, [1/s]

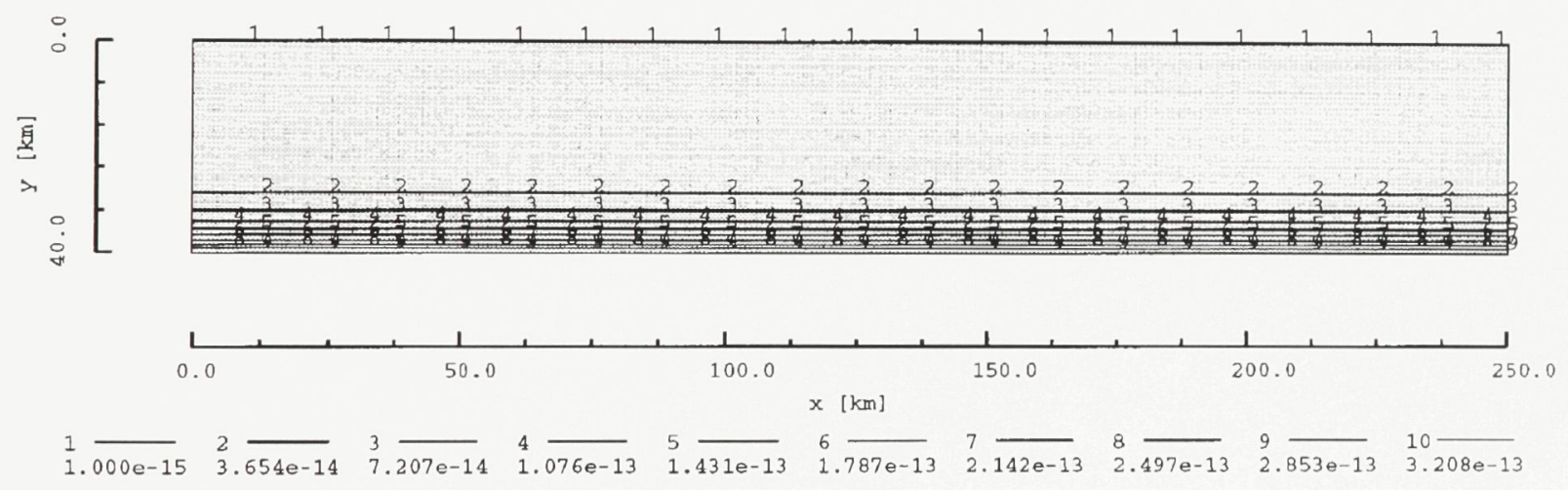

Figure 5.11 (d) tectonic-induced normal and shear stresses along the rear boundary of the block; (e) second invariant of strain rate contour map;

(continued) 

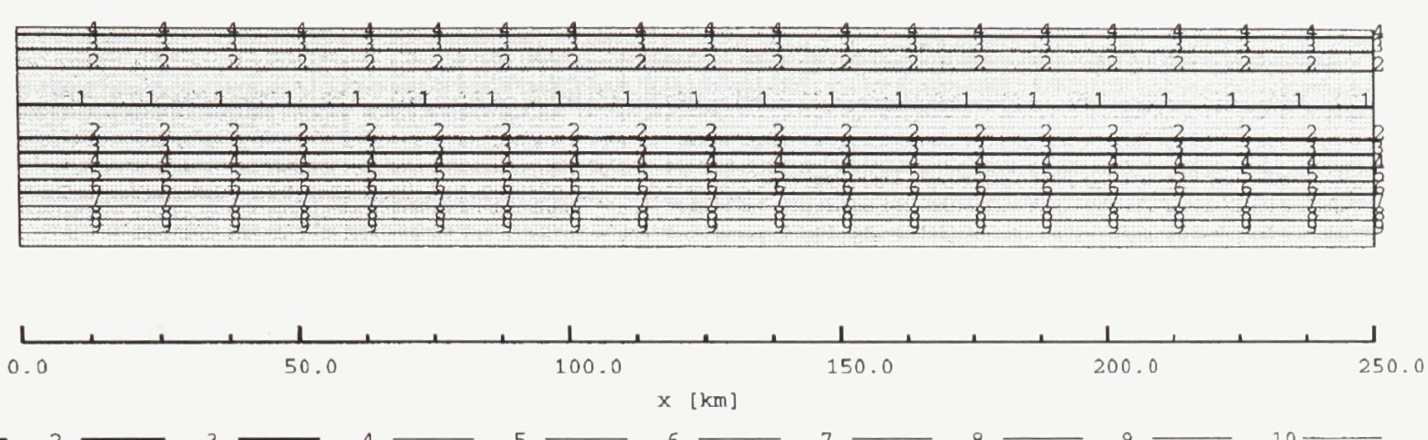

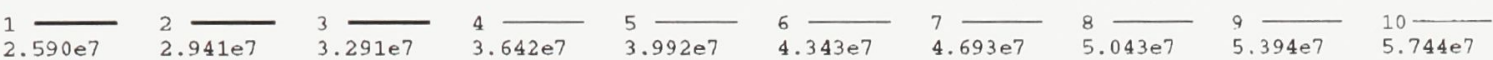

Loglo[efE viscosity] [Pa s]

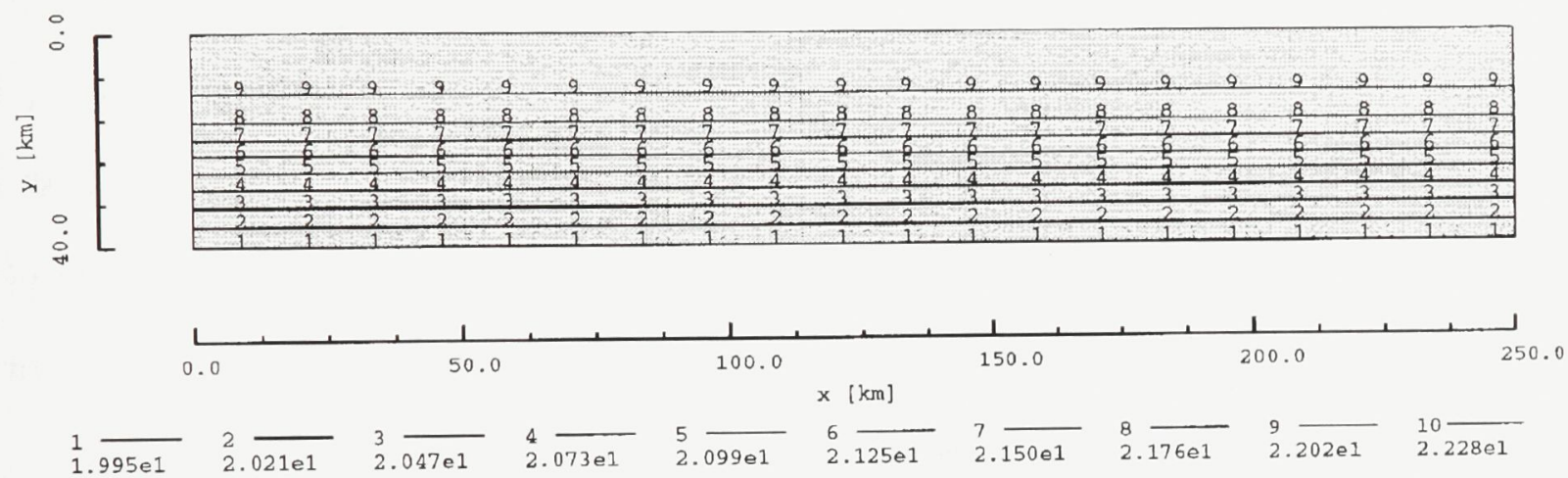

Figure 5.11 (f) second invariant of deviatoric stress contour map; (g) effective viscosity contour map (logarithm to base 10). 
$\mathrm{MPa}$, while in case $\mathrm{E}$ the required compression decreases in magnitude nonlinearly from about $-76 \mathrm{MPa}$ at the top to about $-30 \mathrm{MPa}$ at the bottom of the block.

A constant temperature field of $750 \mathrm{~K}$ has been assumed in case $\mathrm{E}$. In case $\mathrm{F}$, an increase of temperature from the top to the bottom of the block is examined, for the same power-law rheology. The material becomes softer with increasing depth, as the temperature increases from 750 to $850 \mathrm{~K}$ across the thickness of the block. Results for this case are shown in Figure 5.11. Comparing them with Figure 5.10, it is seen that even a rather low temperature gradient increases considerably the ductility of the block material. The effect of temperature is also visible in the stress and strain rate fields (Figures 5.11c, e): predominant laminar flow and high concentration of strain rate are found near the base of the block. The increase in ductility with depth is also reflected in the profile of effective viscosity (Figure 5.11g), which decreases from approximately $1.9 \times 10^{22}$ to $8.9 \times 10^{19} \mathrm{~Pa} \mathrm{~s}$ from the top to the base of the block. The non-zero temperature gradient also affects the depth distribution of the maximum shear stress which, different from that in case E, first decreases towards a minimum (at a depth of about $15 \mathrm{~km}$ ) and then increases with depth (Figure 5.11f). A similar depth distribution of the maximum shear stress is also found in case B (see Figure 5.7f). As in case B, a combination of temperature, rheology, and top longitudinal deformation pattern as specified in case F leads to such a distribution of $F(y)$ that, when combined with the gravitational shear stress $\left(\bar{\rho} g_{x} y\right)$, results in the depth distribution of the maximum shear stress as shown in Figure 5.11f.

Cases A, B, D, E, and F are actually two-dimensional extensions of Nye's (1957) onedimensional model (with linear and nonlinear rheology) and, as discussed previously, reduce to Nye's solution in the one-dimensional case. 


\subsection{Comparison between the two-dimensional power-law solution and Platt's model}

It is interesting to compare the present solution with Platt's dynamic model for orogenic wedges (Platt, 1986), which has been briefly reviewed in section 2.4 of this thesis. It will be shown that Platt's model is a particular case of the present solution for stress.

It should be pointed out that an additional assumption is needed in Platt's model in order to derive equation (2.7). Such an additional assumption, not mentioned in Platt's paper (Platt, 1986), should be that the longitudinal deviatoric stress $\tau_{x x}$ is always equal to zero on the upper surface of the wedge. This assumption is implicitly used when integration with respect to $y$ is performed on the stress equilibrium equation in $x$-direction (see equations 11 and 12 in Platt, 1986). This additional condition means that the nearsurface part of the wedge is always under a lithostatic state of stress, implying that no ductile or brittle deformation occurs in the near-surface area.

In the following, the solution given in equations (5.30) is recast in a format that is directly comparable with Platt's model (i.e., equation 2.7; see Platt, 1986).

Let $\tau_{x x}$ and $\bar{\tau}_{x x}$ be the longitudinal deviatoric stress and the depth-averaged longitudinal deviatoric stress, respectively. It follows from equation (5.8) that

$$
\begin{aligned}
& \tau_{x x}=\frac{F(y)-x G^{\prime}(y)}{2} \\
& \frac{\partial \bar{\tau}_{x x}}{\partial x}=\frac{\partial}{\partial x}\left[\frac{1}{h} \int_{0}^{h} \tau_{x x} d y\right]=-\frac{1}{2 h} \int_{0}^{h} G^{\prime}(y) d y=-\frac{G(y=h)}{2 h}
\end{aligned}
$$

where $h(x)$ is the thickness of the wedge at the point under consideration (see Figure 5.12). The basal traction (shear stress) $\tau_{b}$ is related to the state of stress within the wedge at that point by the following equation ( $c f$. Ranalli, 1995) 


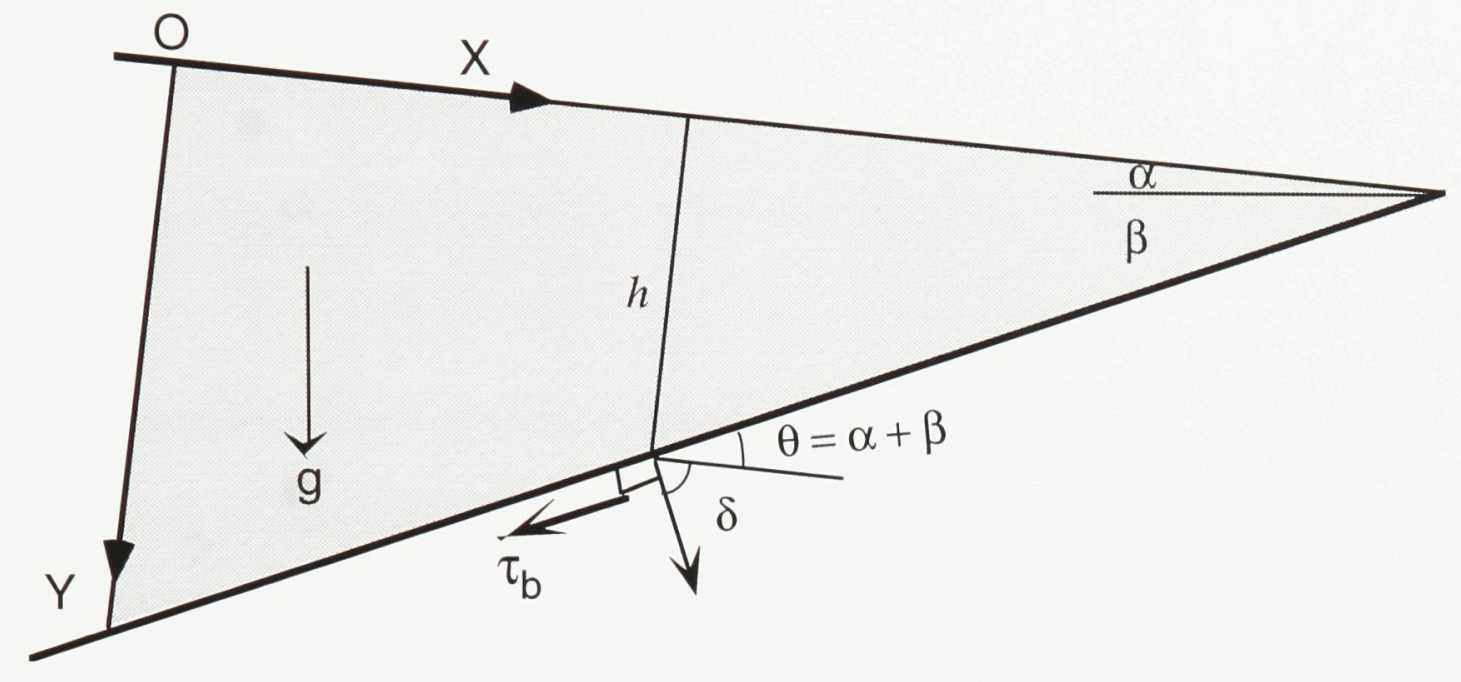

Figure 5.12 Simplified wedge model. $\alpha$ is the surface slope and $\beta$ the basal slope. $\delta$ is the angle between the positive $x$ axis and the normal of the base to the wedge; $\tau_{b}$ is the basal shear stress exerted on the wedge by the subducting basement. 


$$
\begin{aligned}
\tau_{b} & =\frac{1}{2}\left(\sigma_{y y}-\sigma_{x x}\right)_{y=h} \sin 2 \delta+\left(\sigma_{x y}\right)_{y=h} \cos 2 \delta \\
& =\frac{1}{2}\left(\sigma_{y y}-\sigma_{x x}\right)_{y=h} \sin 2 \theta-\left(\sigma_{x y}\right)_{y=h} \cos 2 \theta
\end{aligned}
$$

Combining equations (5.40) and (5.39) leads to

$$
\tau_{b} \approx \bar{\rho} g h \alpha-2 \theta\left(\tau_{x x}\right)_{y=h}+2 h \frac{\partial \bar{\tau}_{x x}}{\partial x}
$$

where small-angle approximations for $\alpha$ and $\theta$ are used.

Comparison of equations (5.41) and (2.7) shows two differences: (1) the signs of the third term on the right side are different; and (2) the stress component in the second term on the right side of equation (5.41) is the longitudinal deviatoric stress on the basement (i.e., $y=h$ )while the corresponding term in equation (2.7) is the depth-averaged longitudinal deviatoric stress. The sign difference is a trivial consequence of the difference in coordinate systems adopted in this thesis and in Platt's model (compare Figures 5.12 and 2.5). The second difference is caused by the aforementioned assumption about the stress state along the wedge upper surface.

Therefore, Platt's (1986) stability criterion is compatible with - indeed, it is a particular case of - the stress solution derived in this chapter, subject to the assumption that the longitudinal deviatoric stress, $\tau_{x x}$, does not vary significantly along any depth profile. This condition most likely holds in thin-skinned wedges but may not be satisfied in thickskinned wedges, where deformation patterns may vary significantly in the vertical direction.

Substituting equations (5.37a) into (5.41), one obtains

$$
\tau_{b} \approx \bar{\rho} g h \alpha-4 \theta\left[a_{2}+b_{2} h+\left(a_{1}+b_{1} h\right) x\right] \eta(h)-4 h \int_{0}^{h}\left(a_{1}+b_{1} y\right) \eta(y) d y
$$


Following the same line of reasoning as in Platt (1986), equation (5.42) implies that a wedge is stable if the following conditions are satisfied

$$
a_{1}=a_{2}=b_{1}=b_{2}=0
$$

Combination of equations (5.43) and (5.30) or (5.37) shows that a wedge in a standard state of stress (where normal stresses are hydrostatic everywhere (Anderson, 1951)) suffers no internal deformation, but can be subject to rigid-body motion (that is, either in translation if the constant $b_{3}=0$ or in rotation if the constants $a_{3}=b_{4}=0$. In general, the motion is a combination of translation and rotation). On the other hand, if conditions (5.43) are not satisfied, the longitudinal deviatoric stress and the corresponding strain rate will not be zero everywhere within the wedge, and the four constants $\left(a_{1}, a_{2}, b_{1}, b_{2}\right)$ will vary in a manner related to the average upper slope $\alpha$ of the wedge, if this is different from the critical taper defined by Platt in equation (2.7).

All the previous discussion is based on the assumption that the basal shear stress $\tau_{b}$ remains unchanged during the tectonic evolution of the wedge. This is particular important, in view of the fact that a change in $\tau_{b}$ has important consequences on the deformational style of orogenic wedges ( $c f$. Willett 1992 and section 2.4 of this thesis). 


\section{Chapter 6}

\section{Orogenic Wedges With Viscous, Power-law, and Plastic Rheologies}

\subsection{Introduction}

Both analytical comparison and numerical computation in Chapter 5 support the following statements: (1) the linear longitudinal strain rate solution (i.e., equations 5.30) is a two-dimensional extension of the classic one-dimensional solution derived by Nye (1957) for ice sheets; (2) some popular qualitative discussions such as Platt's model for orogenic wedges (Platt, 1986) can also be formulated from the two-dimensional solution; (3) two-dimensional blocks (not necessarily rectangular) with generalized power-law rheology and complicated deformation styles can be analyzed by applying the twodimensional linear longitudinal strain rate solution into a "segmentation approximation model" of the block.

In this chapter, this two-dimensional linear longitudinal strain rate solution is applied to study the stress and deformation in orogenic wedges with rheological parameters and temperature values derived from conditions typically found in orogenic belts.

\subsection{Models: geometry, composition and temperature}

The geometry of an orogenic wedge as modeled in this chapter is described by three parameters (Figure 6.1): upper and lower slopes $(\alpha$ and $\beta$ ) and the width of the wedge $(L)$. For simplicity, the slope $\alpha$ is assumed to be constant throughout the whole wedge in all the computations, although a variable $\alpha$ can be accommodated through segmentation 
of a large wedge block (see Figure 5.2). Similarly a constant basal slope $\beta$ is assumed in all computations. All computation results are presented within a global coordinate system which is defined with its origin $O$ fixed at the rear top corner of wedge in its initial position and with positive $x$ axis parallel to the wedge top surface and directed towards the front, while the positive $y$ axis is perpendicular to the top surface and pointing to the basement. This coordinate system is termed global as against those local ones defined in each wedge segment (see Figure 5.3). The basal shear stress $\tau_{b}$ is specified as part of the boundary conditions.

The petrological composition of orogenic wedges is variable. In general, orogenic wedges should have a bulk composition approximating that of continental crust (Pavlis and Bruhn, 1983). All model computations presented in this chapter assume orogenic wedges to consist of rocks with the rheology of quartzite (soft end of the spectrum) and anorthosite (hard end of the spectrum).

Fluid movement within orogenic wedges plays an important role in determining the overall dynamic behaviour during tectonic evolution (von Huene, 1984; Vrolijk, 1987; Fisher and Byrne, 1990; Bebout, 1991). The importance of fluid for rock deformation in the brittle regime is well known (Jaeger and Cook, 1969; Dahlen, 1990; Ranalli, 1995), and it can also affect the bulk ductile rheology: wet and dry rocks of the same type have different values of bulk rheological parameters (Table 6.1). The role of fluid on the dynamics of orogenic wedges is studied in this chapter by choosing both wet and dry rocks as constituents of the wedges.

The magnitude of strain rate in orogenic complexes is generally estimated to be between $10^{-13}$ and $10^{-15} \mathrm{~s}^{-1}$ (Pfiffner and Ramsay, 1982; Ranalli and Murphy, 1987; Twiss and Moores, 1992, p. 382; Crespi et al., 1996). 


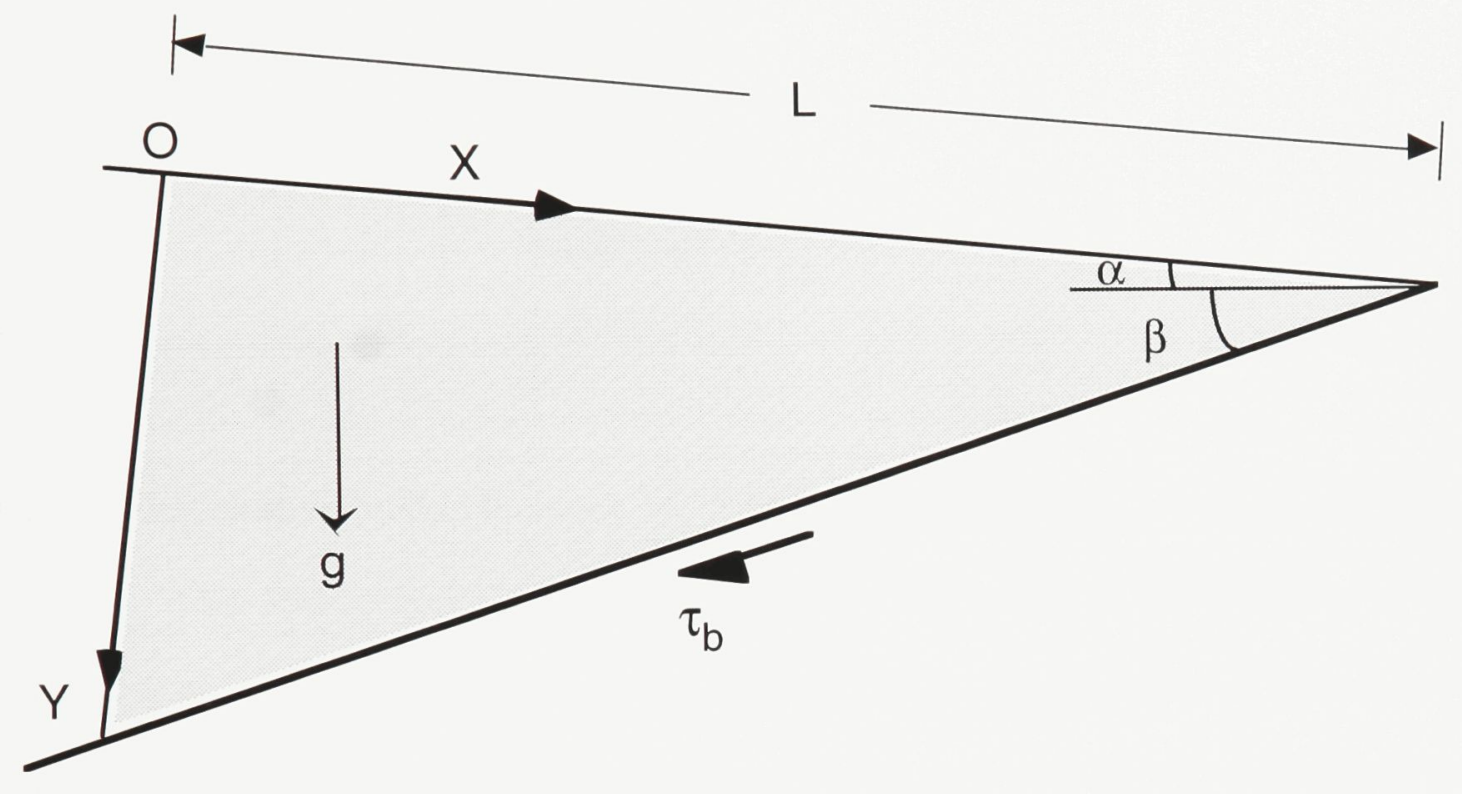

Figure 6.1 An orogenic wedge model parameterized by surface slope $(\alpha)$, basal slope $(\beta)$ and width $(L)$. Basal shear stress and gravity are denoted by $\tau_{b}$ and $g$, respectively. 
Table 6.1 Steady-state power-law creep parameters for crustal materials (Ranalli \& Murphy, 1987; data modified from Kirby, 1983)

\begin{tabular}{lccc}
\hline Rock Material & $A_{0}\left(\mathrm{~Pa}^{-n} \mathrm{~s}^{-1}\right)$ & $n$ & $Q\left(\mathrm{~kJ} \mathrm{~mol}^{-1}\right)$ \\
\hline rocksalt & $9.976 \times 10^{-32}$ & 5.3 & 102 \\
granite & $7.924 \times 10^{-29}$ & 3.2 & 123 \\
granite (wet) & $7.943 \times 10^{-16}$ & 1.9 & 137 \\
quartzite & $2.512 \times 10^{-20}$ & 2.4 & 156 \\
quartzite (wet) & $3.990 \times 10^{-18}$ & 2.3 & 154 \\
albite rock & $1.033 \times 10^{-29}$ & 3.9 & 234 \\
anorthosite & $2.060 \times 10^{-23}$ & 3.2 & 238 \\
quartz diorite & $5.024 \times 10^{-18}$ & 2.4 & 219 \\
diabase & $8.038 \times 10^{-25}$ & 3.4 & 260 \\
\hline
\end{tabular}

Another important factor affecting the rheological behaviour is the thermal regime of the wedges. The importance of temperature in rock deformation can be seen from equations (4.4) and (4.5) which shows explicitly the dependence of the power-law relation between effective shear strain rate and effective shear stress on the temperature. The temperature distribution in the lithosphere can be formulated by the heat conduction equation ( $c f$. Ranalli, 1995)

$$
K \nabla^{2} T=c \rho \frac{\partial T}{\partial t}-H_{G}
$$

where $c$ and $\rho$ are the specific heat and density, respectively; $K$ is the thermal conductivity and $H_{G}$ the heat generation rate per unit volume. Assuming, as a first approximation, a steady-state thermal regime, a constant heat-production function $H_{G}$ and depth-dependent-only temperature distribution, the solution to equation (6.1) is

$$
T(z)=T_{0}+\frac{q_{0}}{K} z-\frac{H_{G}}{2 K} z^{2}
$$


where $T_{0}$ and $q_{0}$ are surface temperature and heat flow, respectively, and $z$ is depth.

In a 'typical' accretionary wedge, observed heat flow and heat-production are about $30-40 \mathrm{~mW} \mathrm{~m}^{-2}$ and $1 \mu \mathrm{W} \mathrm{m}^{-3}$, respectively (Pavlis and Bruhn, 1983). Heat flow observations and knowledge of thermal properties can be used to calculate geotherms in orogenic wedges ( $c f$. e.g. Hyndman and Wang, 1993). In most of the computations presented in this chapter, a linear depth distribution of temperature is assumed, and the effects of temperature on deformation are studied by considering both 'cool' and 'hot' wedges (i.e., wedges with low and high geothermal gradients).

In the following sections of this chapter, models are presented in two groups: (a) the rheology of the whole wedge is viscous power-law or linear creep; and (b) the rheology of the wedge, controlled mainly by the temperature and rock type, is perfectly plastic in the upper part and power-law in the lower part.

\subsection{Orogenic wedges with linearly viscous and power-law rheologies}

Following the same procedure used in Section 5.5, the linear longitudinal strain rate solution (i.e., equation 5.30) is applied to investigate the stress and deformation of orogenic wedges subject to certain force and velocity boundary conditions which are specified the same way as in Chapter 5 (see equations 5.38). The three cases considered in this section differ from those studied in Section 5.5 in that shear stress is specified along the wedge basement, and the latter is not parallel to the top surface. The parameter values of these three cases are listed in Table 6.2. The basal shear stress is specified such that for all three cases it decreases in magnitude linearly from -80 MPa at the rear to zero at the front of wedge. A constant density of $2700 \mathrm{~kg} \mathrm{~m}^{-3}$ is assumed, given the minor effects of variations of density on the dynamics of orogenic wedges. The specification of 
the $v$ component of the top velocity boundary condition is also the same in all three cases, which is $-5 \mathrm{~mm} \mathrm{a}^{-1}$ (upward) at the rear and zero at the front of the wedge. Attention is focused on the stress and deformational characteristics of orogenic wedges with different homogeneous rheologies and different longitudinal deformation patterns on their top surface.

Table 6.2 Model parameters for orogenic wedges with width $L=200 \mathrm{~km}$ and top and basal slopes $\alpha=3^{\circ}$ and $\beta=6^{\circ}$ (constant density $\rho=2700 \mathrm{~kg} \mathrm{~m}^{-3}$ )

Case*

\begin{tabular}{|c|c|c|c|}
\hline \multirow{2}{*}{ Parameter } & \\
\hline & A1 & B1 & $\mathrm{C} 1$ \\
\hline$u_{0}\left(\times 10^{-10} \mathrm{~m} \mathrm{~s}^{-1}\right)$ & 0 & 0 & 0 \\
\hline$u_{1}\left(\times 10^{-15} \mathrm{~s}^{-1}\right)$ & -1 & -1 & 1 \\
\hline$u_{2}\left(\times 10^{-20} \mathrm{~m}^{-1} \mathrm{~s}^{-1}\right)$ & -2.25 & -2.25 & -1 \\
\hline$v_{0}\left(\times 10^{-10} \mathrm{~m} \mathrm{~s}^{-1}\right)$ & -1.5855 & -1.5855 & -1.5855 \\
\hline$v_{1}\left(\times 10^{-15} \mathrm{~s}^{-1}\right)$ & 0.7927 & 0.7927 & 0.7927 \\
\hline$\tau_{0}(\mathrm{MPa})$ & -80 & -80 & -80 \\
\hline$\tau_{1}\left(\mathrm{MPa} \mathrm{km}^{-1}\right)$ & 0.4 & 0.4 & 0.4 \\
\hline$T_{0}(\mathrm{~K})$ & $\S$ & 750 & 750 \\
\hline$T_{1}\left(\mathrm{~K} \mathrm{~km}^{-1}\right)$ & & 0 & 0 \\
\hline$A_{0}^{*}\left(\times 10^{-23} \mathrm{~Pa}^{-1} \mathrm{~s}\right)$ & 5 & & \\
\hline$A_{1}^{*}\left(\times 10^{-23} \mathrm{~Pa}^{-1} \mathrm{~s}^{-1} \mathrm{~km}^{-1}\right)$ & 0 & & \\
\hline power-law rheology & & \multicolumn{2}{|c|}{ anorthosite $\dagger$} \\
\hline
\end{tabular}

* Cases A1, B1, and C1 refer to Figures 6.2, 6.3, and 6.4

A1: Newtonian, constant $\eta\left(=10^{22} \mathrm{Pas}\right.$ ), variable $\dot{\varepsilon}_{x x}$ and variable $v$ on surface, variable $\tau_{b}$

B1: Power-law, constant $T$, variable $\dot{\varepsilon}_{x x}$ (always negative) and variable $v$ on surface, variable $\tau_{b}$

C1: Power-law, constant $T$, variable $\dot{\varepsilon}_{x x}$ (positive and negative) and variable $v$ on surface, variable $\tau_{b}$

$\S$ empty entry indicates that parameter is not applicable

$\dagger$ rheological parameters: $A_{0}=2.06 \times 10^{-23}\left(\mathrm{~Pa}^{-3.2} \mathrm{~s}^{-1}\right), n=3.2, Q=238\left(\mathrm{~kJ} \mathrm{~mol}^{-1}\right)$

Case A1 (Figure 6.2) is a wedge with constant Newtonian viscosity $\left(\eta=10^{22} \mathrm{~Pa} \mathrm{~s}\right)$ and such a longitudinal component of the top velocity boundary condition that the 
longitudinal strain rate $\dot{\varepsilon}_{x x}$ varies from $-10^{-15} \mathrm{~s}^{-1}$ at the rear to $-10^{-14} \mathrm{~s}^{-1}$ at the front of the wedge. The dominant movement pattern of the wedge material is longitudinal at the front and the vertical movement becomes more significant when approaching the rear of the wedge (Figure 6.2a). The maximum shear stress trajectories (Figure 6.2b) indicate a predominant thrusting deformation regime throughout the wedge. The effective shear strain rate $\dot{\varepsilon}_{E}^{\prime}$ (Figure 6.2c) varies mainly in the longitudinal direction. The spatial distribution of the effective shear stress $\sigma_{E}^{\prime}$ (Figure 6.2d) is identical to that of the effective strain rate, increasing from $20 \mathrm{MPa}$ at the rear to $200 \mathrm{MPa}$ at the front of the wedge. $F(y)$, the nongravitational normal stress at the rear of the wedge necessary to maintain the deformation regime (Figure 6.2e) decreases from $-40 \mathrm{MPa}$ at the top to -50 $\mathrm{MPa}$ at the rear bottom of the wedge. $G(y)$, the nongravitational shear stress at the rear of the wedge, varies roughly in a parabolic way with the depth.

Results for case B1 are shown in Figure 6.3. Since cases A1 and B1 have the same force and velocity boundary condition, comparison between the two shows the difference, in terms of stress, strain rate and velocity fields, between wedges with constant Newtonian viscosity and wedges with power-law creep rheology. The rheology of the wedge material in case B1 is that of anorthosite under constant temperature of $750 \mathrm{~K}$ (see Table 6.2). The velocity field (Figure 6.3a) is similar to that of case A1. The slopes of the maximum shear stress trajectories (Figure 6.3b) are slightly flatter in case B1 than in case A1, especially in the rear lower part of the wedge. The effective shear strain rate $\dot{\varepsilon}_{E}^{\prime}$ (Figure 6.3c) varies mainly in the longitudinal direction in the upper part of the wedge and varies significantly in the vertical direction in the rear lower part of the wedge. The spatial distributions of the effective shear stress $\sigma_{E}^{\prime}$ (Figure 6.3d) and the effective viscosity (Figure 6.3e) are similar to that of the effective shear strain rate. At the rear of the wedge, the effective viscosity decreases from $1.8 \times 10^{22} \mathrm{~Pa} \mathrm{~s}$ at the top to 

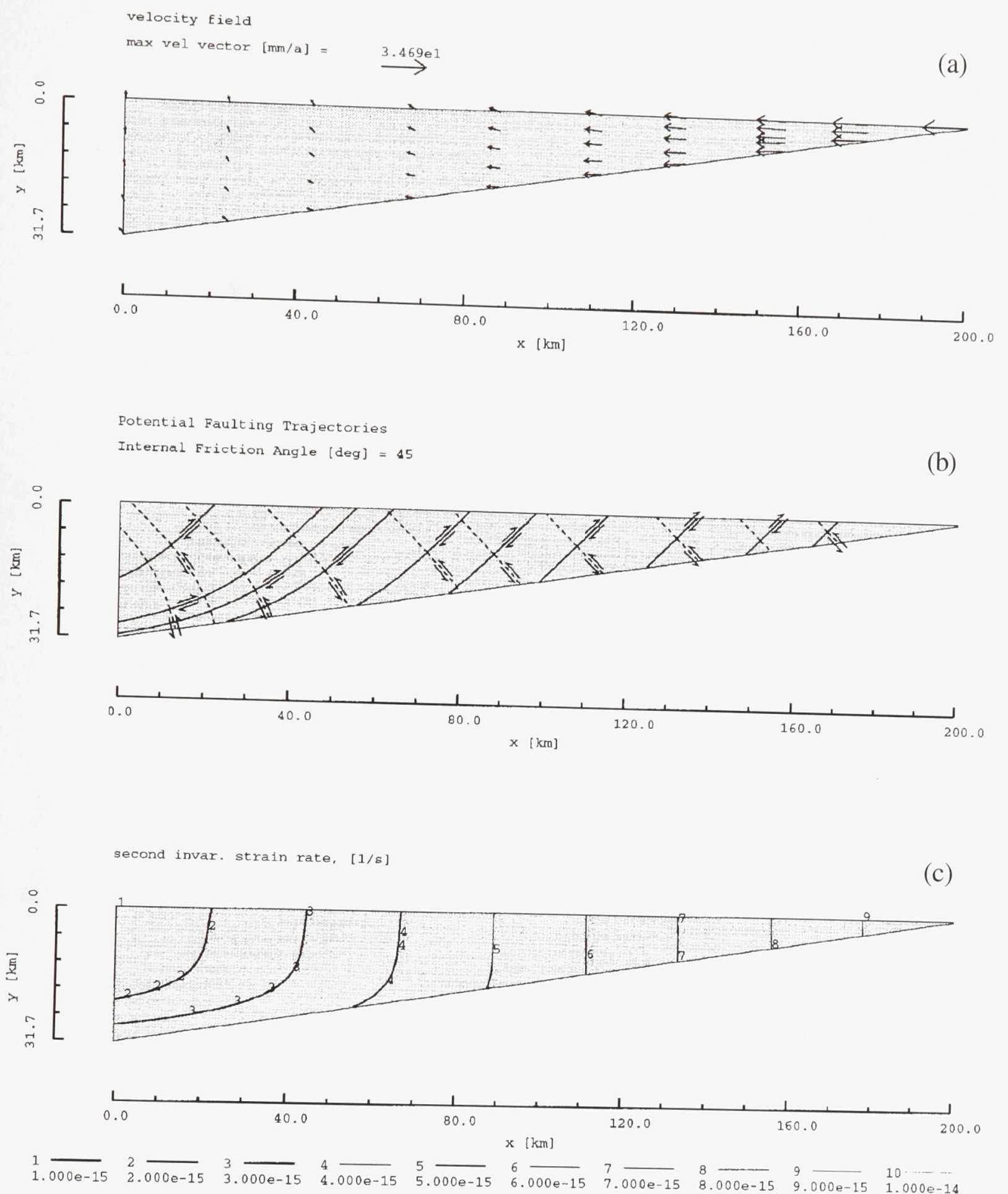

Figure 6.2 Stress and deformation within the wedge for case A1 of Table 6.2. (a) Velocity field; (b) maximum shear stress trajectories; (c) second invariant of strain rate tensor; (continued) 

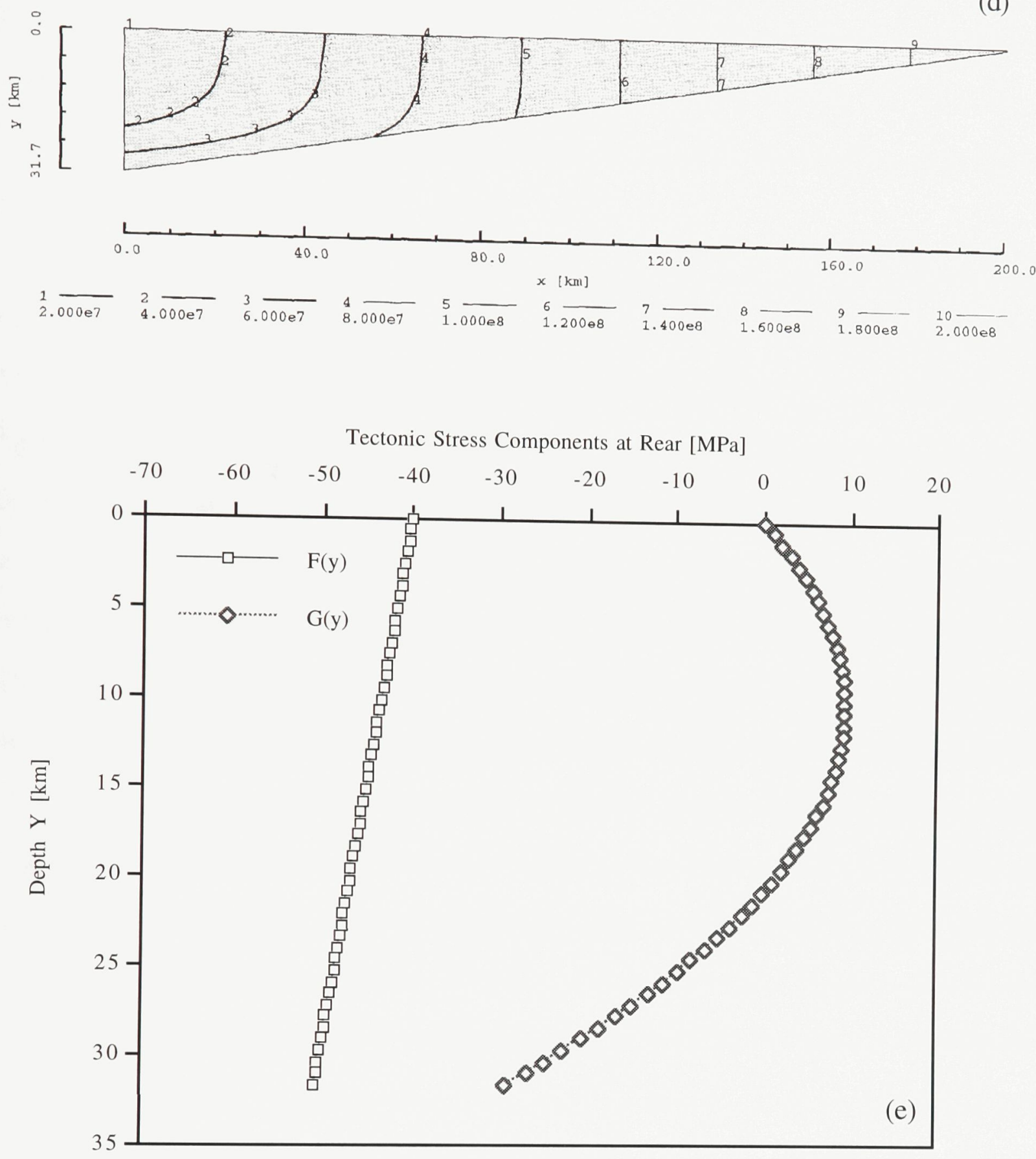

Figure 6.2 (d) second invariant of deviatoric stress tensor; (e) tectonic-induced normal and shear stresses along the rear boundary of the wedge necessary to maintain the deformation. 
$3.8 \times 10^{21} \mathrm{~Pa} \mathrm{~s}$ at the bottom of the wedge. The nongravitational normal stress at the rear of the wedge necessary to maintain this deformation regime (Figure 6.3f) decreases nonlinearly from about -72 MPa at the top to about $-15 \mathrm{MPa}$ at the bottom of the wedge, differing significantly from that in case A1. The depth distribution of the nongravitational shear stress at the rear of the wedge is similar in both cases.

Case C1 (Figure 6.4) differs from case B1 by the longitudinal component of the velocity boundary condition specified on the top of the wedge. The longitudinal strain rate $\dot{\varepsilon}_{x x}$ on the top surface of the wedge varies linearly from $10^{-15} \mathrm{~s}^{-1}$ at the rear to $-3 \times 10^{-15} \mathrm{~s}^{-1}$ at the front. The velocity field (Figure 6.4a) is significantly different from that in case B1. The magnitude of velocity vector increases with depth in the rear part of the wedge. The maximum shear stress trajectories (Figure 6.4b) show coeval development of an extensional regime (normal faulting) at the rear and a compressional regime (thrusting) at the front of the wedge. The effective shear strain rate $\dot{\varepsilon}_{E}^{\prime}$ (Figure 6.4c) varies mainly in the vertical direction and is highly concentrated in the rear lower part of the wedge. The spatial distributions of the effective shear stress $\sigma_{E}^{\prime}$ (Figure 6.4d) and the effective viscosity (Figure 6.4e) are similar in that both show a wedge having an upper region with mainly lateral variation and a lower region with dominantly vertical variation. The nongravitational normal stress at the rear of the wedge necessary to maintain this deformation regime is extensional; $F(y)$ (Figure 6.4f) decreases nonlinearly from about $80 \mathrm{MPa}$ on the top to about $10 \mathrm{MPa}$ at the bottom of the wedge. The depth distribution of the nongravitational shear stress at the rear of the wedge, $G(y)$, is similar to both cases A1 and B1. 


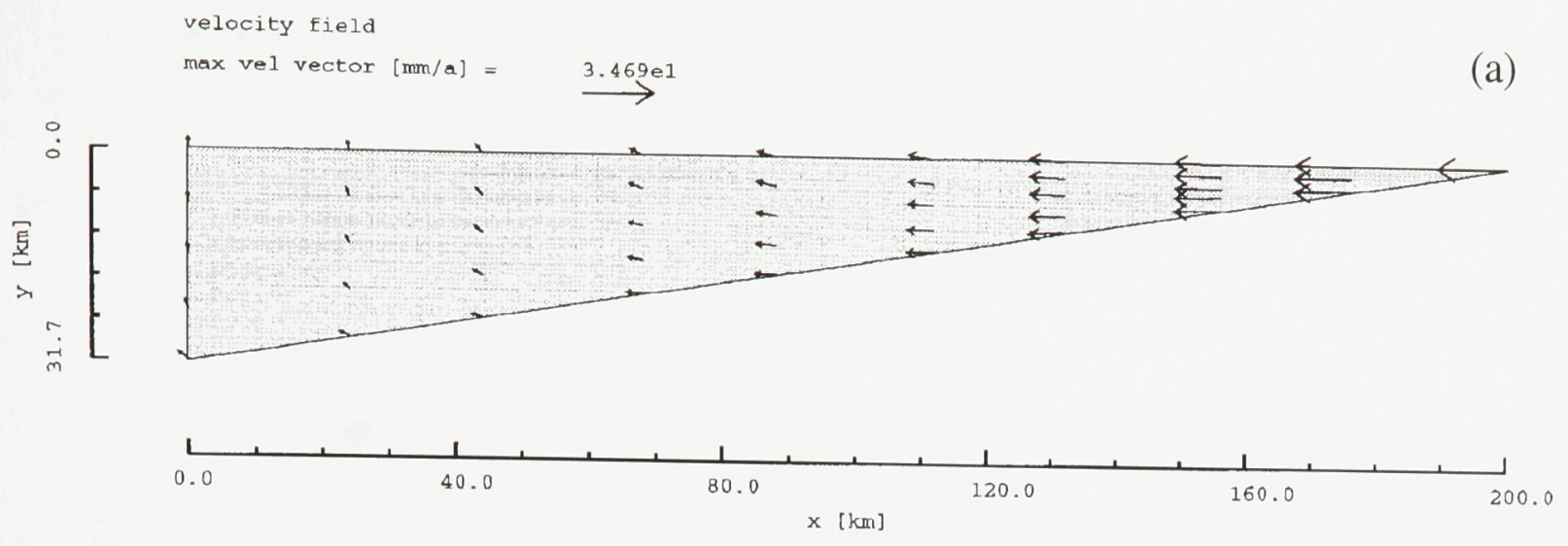

Potential Faulting Trajectories

Internal Friction Angle [deg] $=45$

(b)
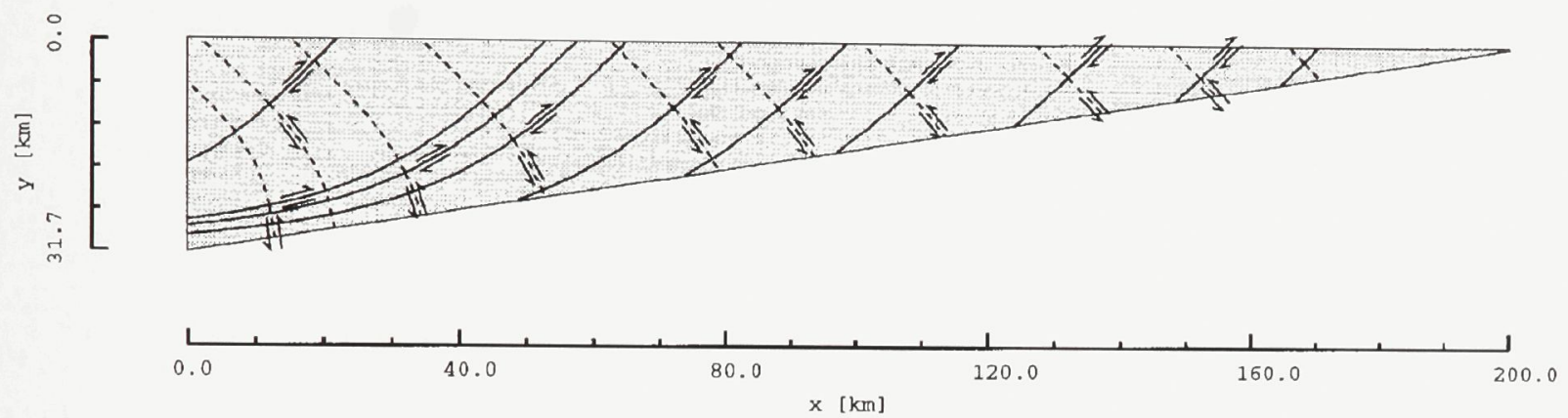

second invar. strain rate, [1/s]

(c)
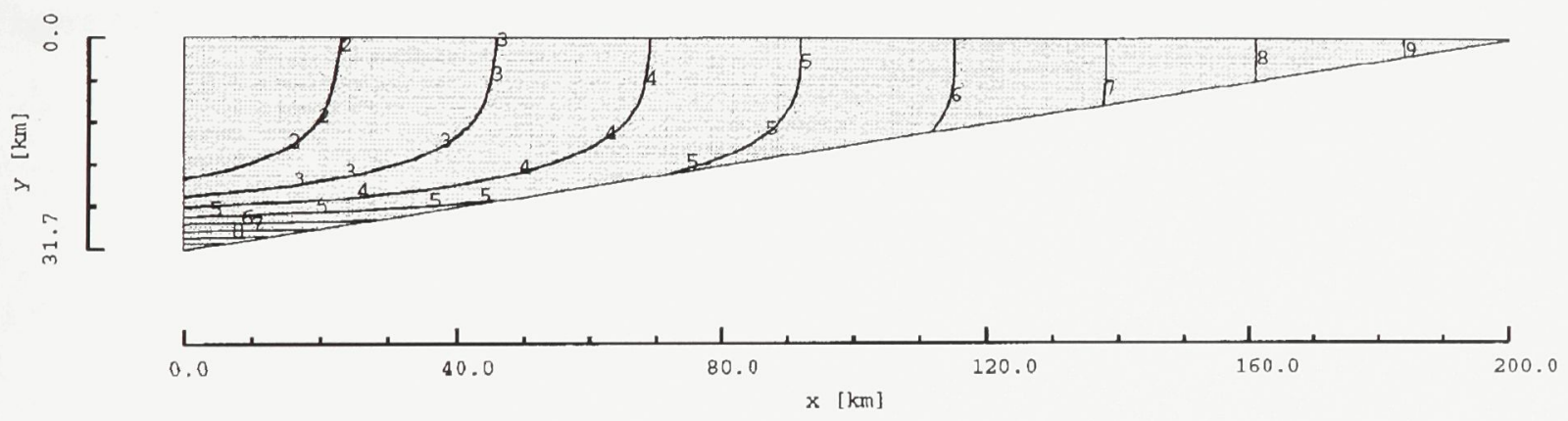

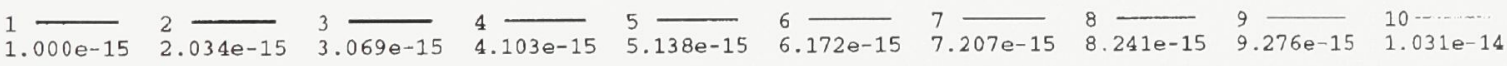

Figure 6.3 Stress and deformation within the wedge for case B1 of Table 6.2. (a) Velocity field; (b) maximum shear stress trajectories; (c) second invariant of strain rate tensor;

(continued) 


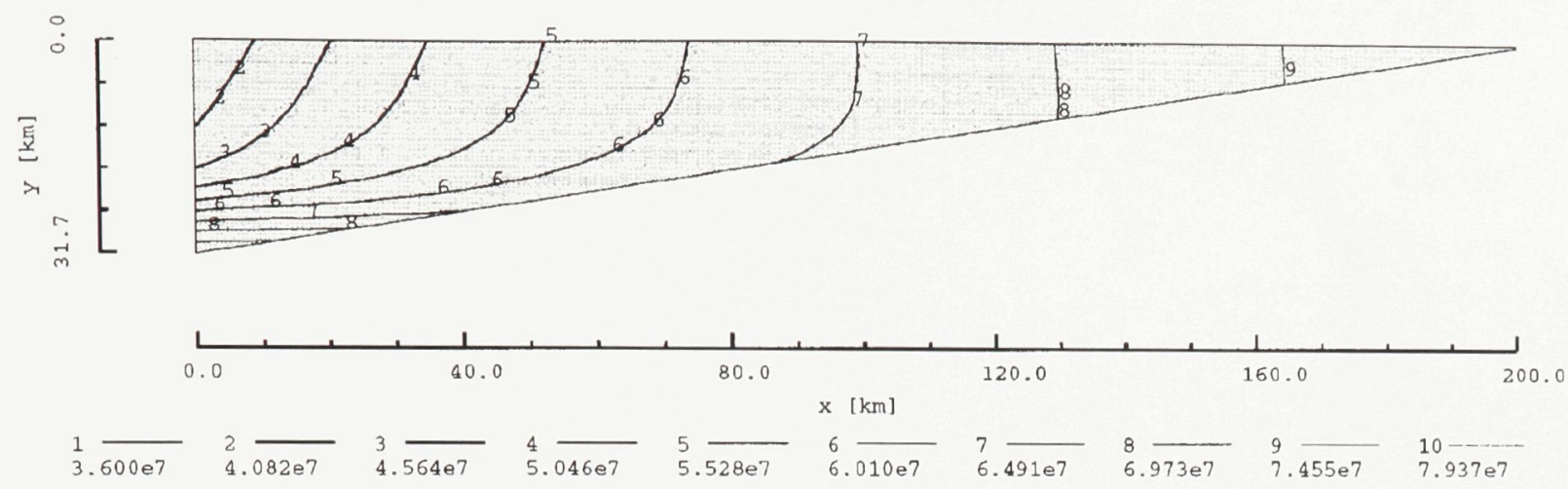

$\log 10[$ eff viscosity] [Pa s]

(e)

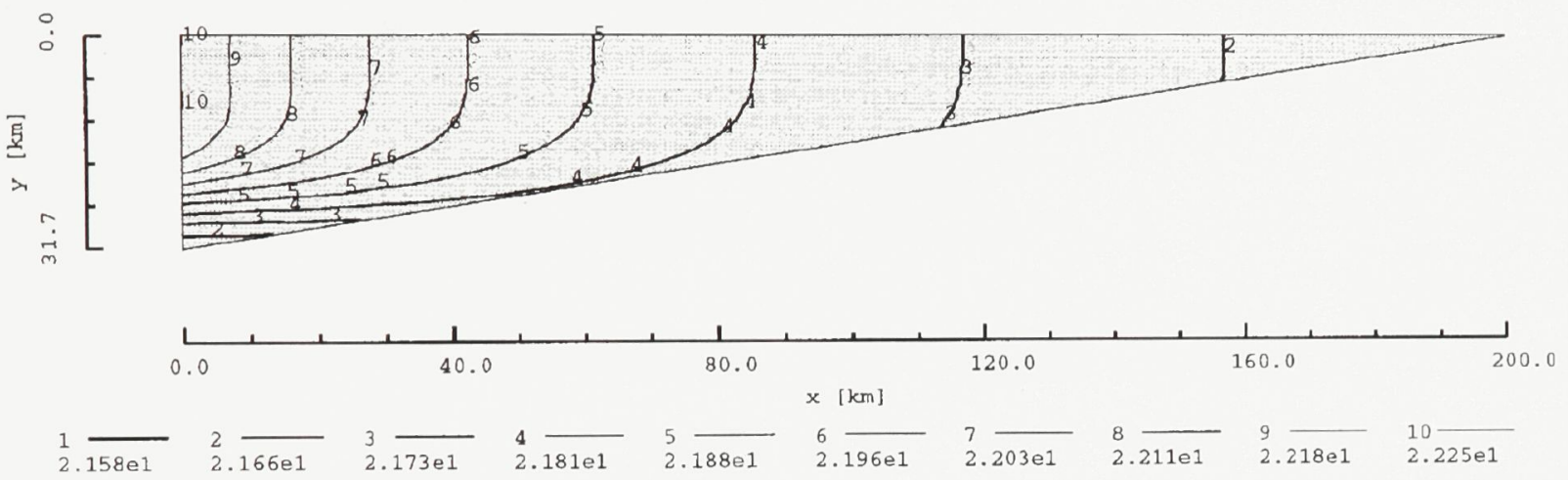

Figure 6.3 (d) second invariant of deviatoric stress tensor; (e) effective viscosity;

(continued) 


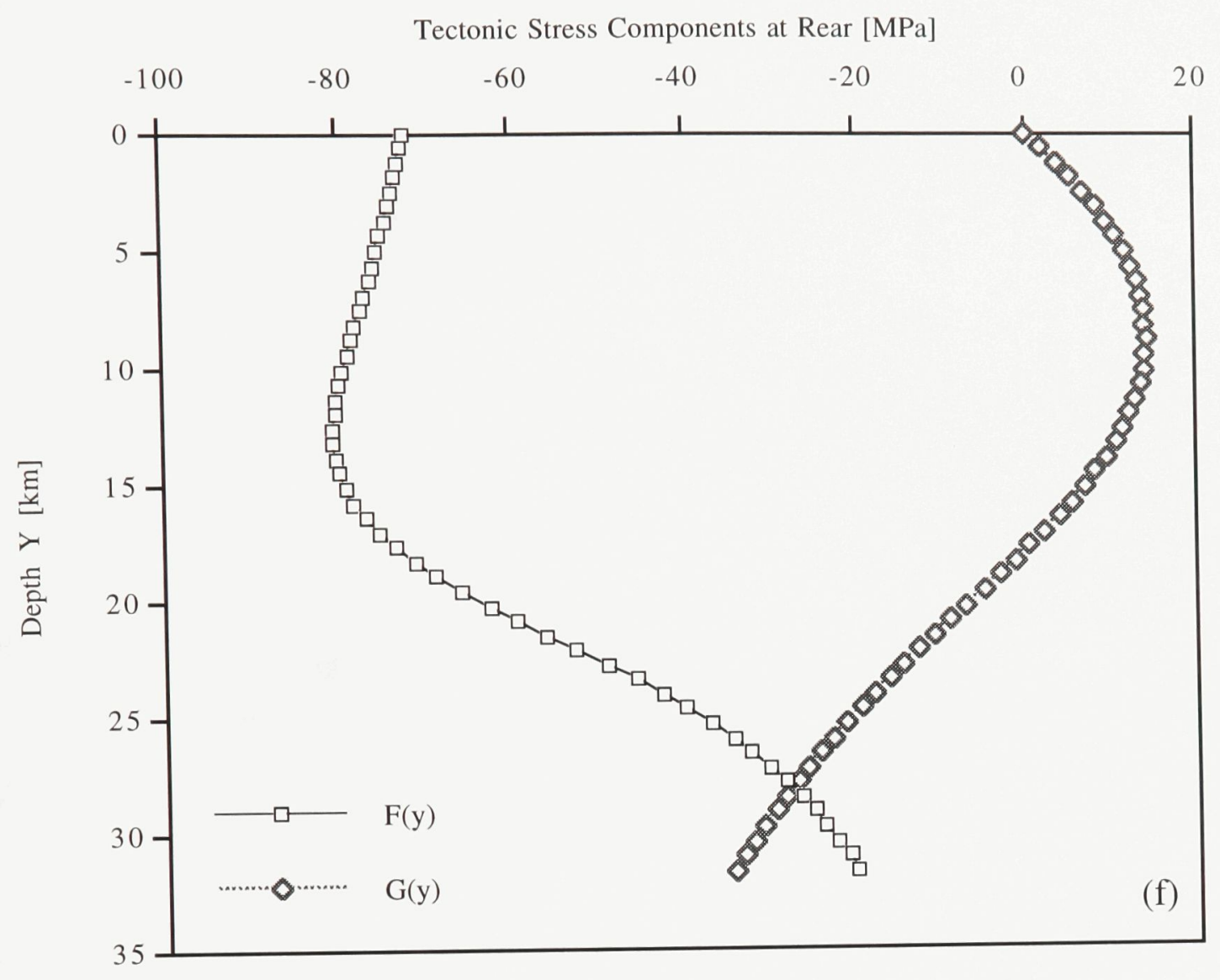

Figure 6.3 (f) tectonic-induced normal and shear stresses along the rear boundary of the wedge necessary to maintain the deformation. 


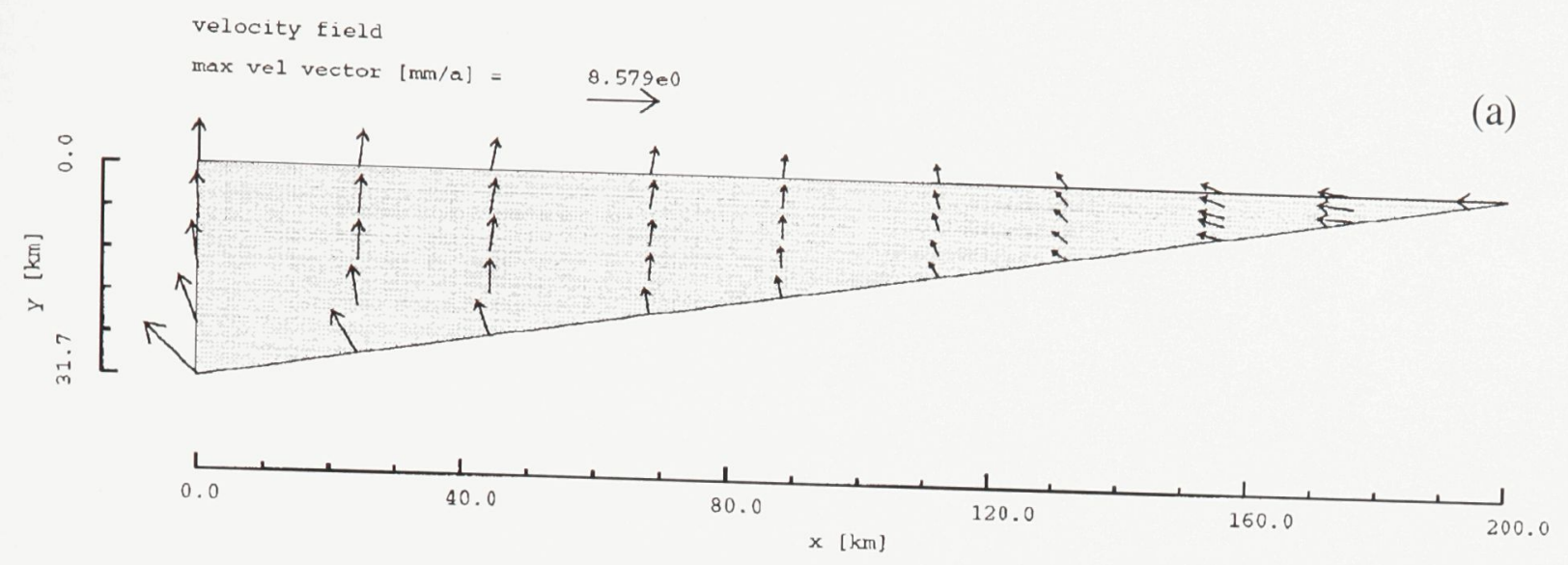

Potential Faulting Trajectories

Internal Friction Angle [deg] $=45$

(b)
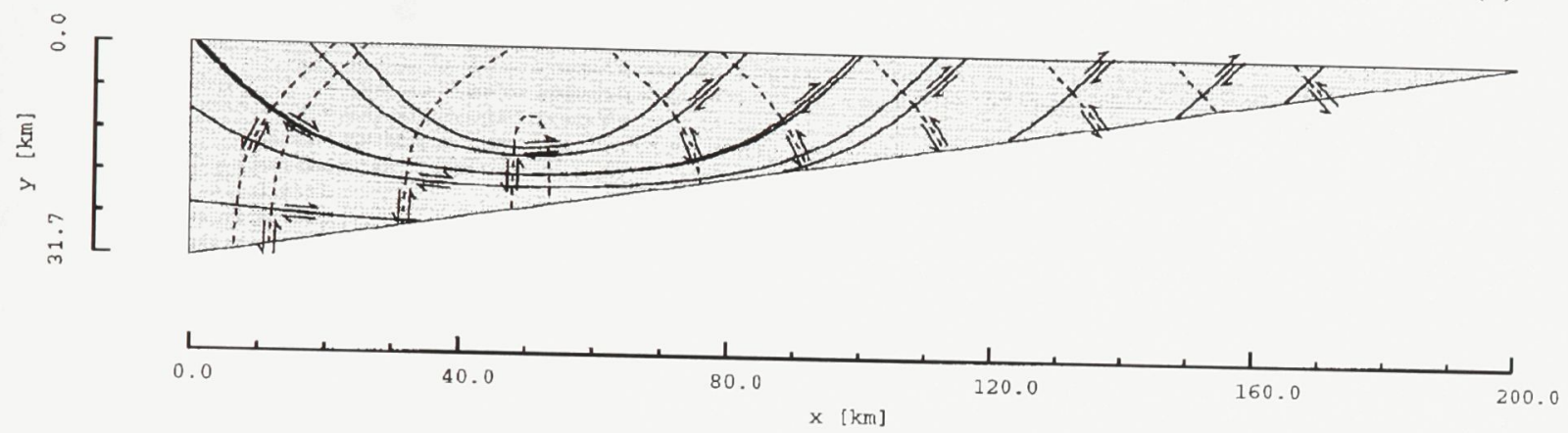

second invar. strain rate, [1/s]

(c)
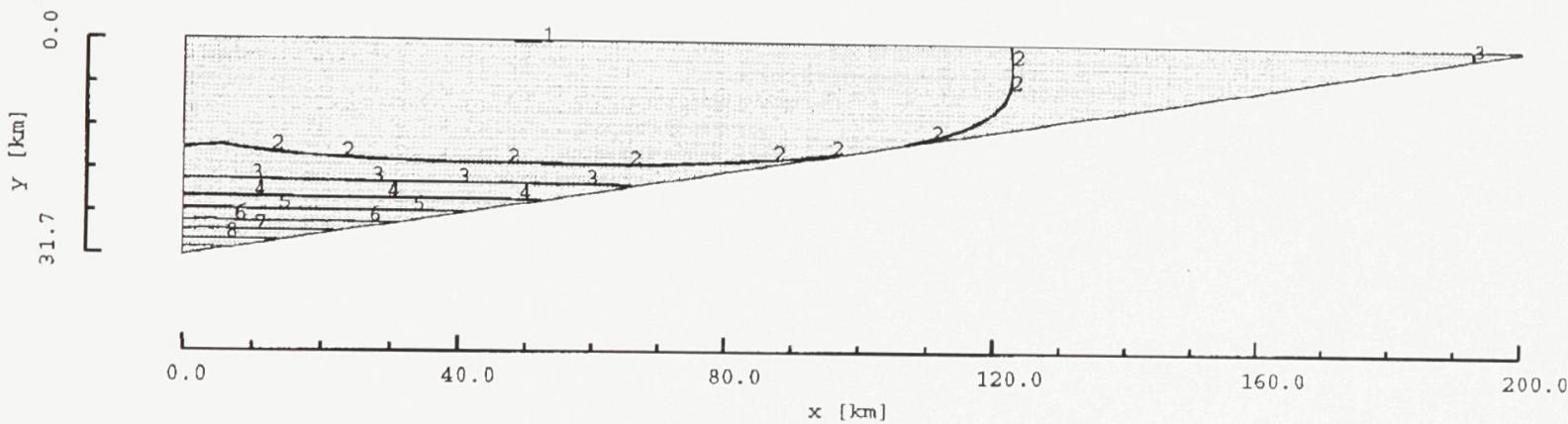

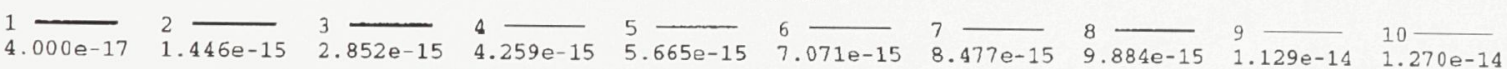

Figure 6.4 Stress and deformation within the wedge for case C1 of Table 6.2. (a) Velocity field; (b) maximum shear stress trajectories; (c) second invariant of strain rate tensor; (continued) 


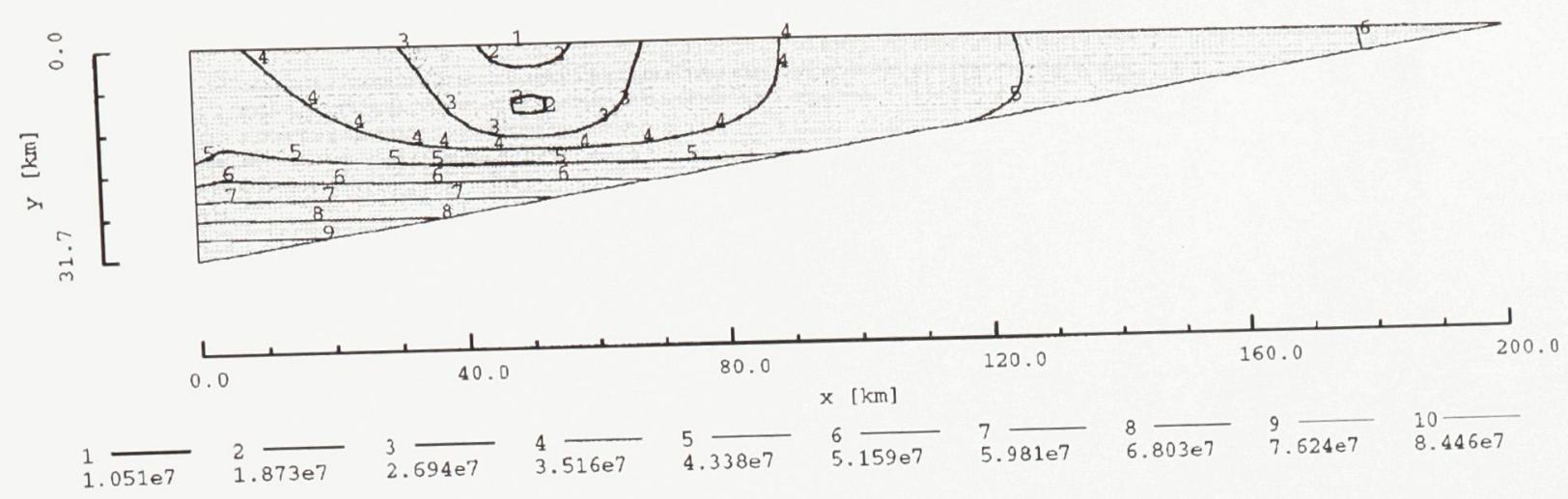

Log10[eff viscosity] [Pa s]

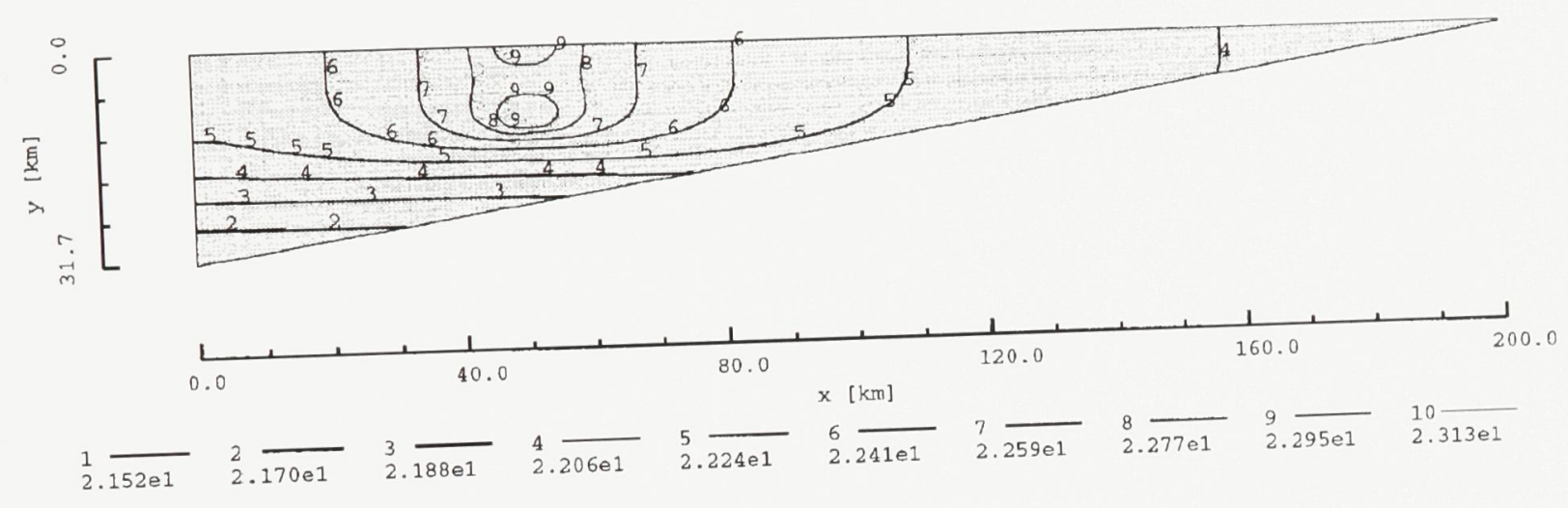

Figure 6.4

(d) second invariant of deviatoric stress tensor; (e) effective viscosity; (continued) 


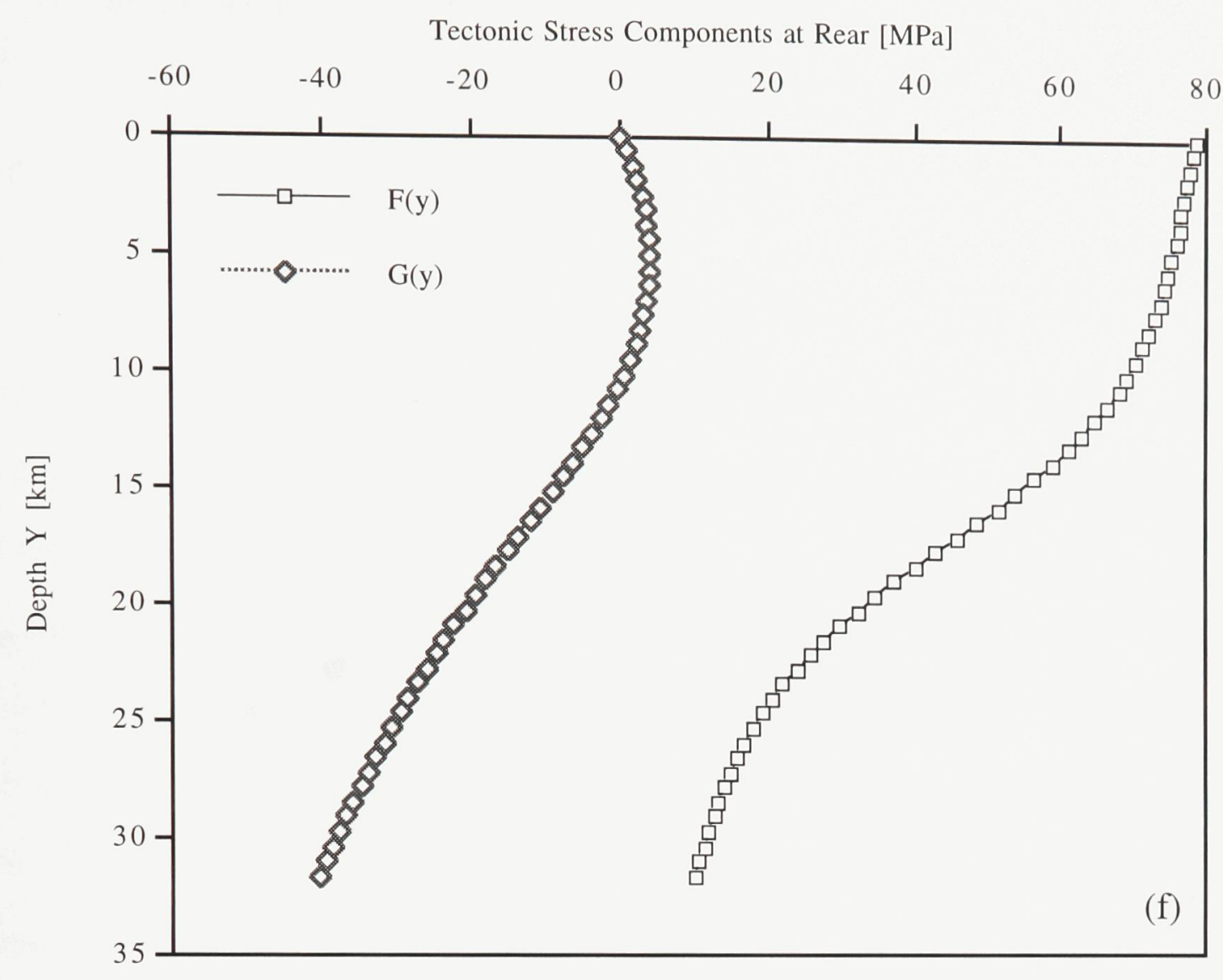

Figure 6.4 (f) tectonic-induced normal and shear stresses along the rear boundary of the wedge necessary to maintain the deformation. 


\subsection{Orogenic wedges with viscoplastic rheology}

\subsubsection{Low-temperature plastic upper layer}

Deformation within orogenic wedges varies not only in the longitudinal direction as discussed in Section 2.6, but also in the vertical direction (mainly due to the change in rheology of rock material). Crustal materials behave differently under various pressure and temperature conditions. The stress difference $\left(\sigma_{1}-\sigma_{2}\right)$ of geodynamic interest is in the range 1- $100 \mathrm{MPa}$ (Ranalli, 1995). At this stress level, bulk rheology of crustal rocks is mainly controlled by temperature which, as a first approximation, increases linearly with depth. Deformation of crustal rock is mainly frictional-plastic at shallow depth (where temperature is low); power-law rheology becomes more dominant when temperature increases (i.e., deeper in crust). Therefore, the whole wedge can be roughly divided into two regions: upper plastic and lower power-law creep domains (Figure 6.5). The critical temperature $\left(T_{c}\right)$ boundary separating frictional-plastic and power-law creep regimes is roughly half of the solidus temperature $\left(T_{m}\right)$ of the material, which is itself pressure-dependent. As a first approximation, we take $T_{c}$ to be $600 \mathrm{~K}$ for quartzite (550 $\mathrm{K}$ if the material is wet), and $700 \mathrm{~K}$ for anorthosite. (Constraints on these values come from the depth distribution of seismicity; $c f$. Ranalli, 1993.)

A general power-law rheology as formulated in equations (4.2) - (4.5) provides a uniform expression which, when the stress exponent $n$ varies, may represent both plastic and viscous rheology (see Table 4.1). This is also shown by the stress-strain rate curves in Figure 6.6. Within the framework of general power-law rheology as adopted in this thesis, the upper frictional-plastic region can be approximated as perfectly plastic (that is, no deformation if the effective shear stress $\sigma_{E}^{\prime}$ is less than the plastic strength $\sigma_{Y}$; plastic deformation when $\sigma_{E}^{\prime}$ equals $\sigma_{Y}$ ). 
Recalling that the effect of rheology on the dynamics of the wedge is realized through the function $F(y)$ (equation 5.34), there are two different ways to simulate the plastic behaviour in upper part of the wedge, namely, (a) by replacing the stress exponent $n$ in the upper part of the wedge with a larger value and then calculate $F(y)$; or (b) by replacing equation (5.34) with another relation (i.e., Mises-Hencky criterion, $c f$. Reiner, 1960) which models the perfectly plastic flow. The latter approach is adopted in the computations to be discussed in this chapter. The effective shear stress $\sigma_{E}^{\prime}$ can be expressed in terms of the effective viscosity and other parameters by the following equation (see equation 5.34)

$$
\sigma_{E}^{\prime}=\left\{4\left[a_{2}+b_{2} y+\left(a_{1}+b_{1} y\right) w\right]^{2} \eta(y)^{2}+\left[\int_{0}^{y}\left[\rho(\tau) g_{x}-4\left(a_{1}+b_{1} \tau\right) \eta(\tau)\right] d \tau\right]^{2}\right\}^{\frac{1}{2}}
$$

where all the symbols have the same meanings as in equation (5.34). Assuming that the strength of rock material in the upper part of the wedge can be expressed as a function of depth such as $s(y)$, the Mises-Hencky criterion for perfectly plastic flow as a function of depth becomes

$$
\begin{aligned}
{[s(y)]^{2} } & =\sigma_{E}^{\prime 2} \\
& =4\left[a_{2}+b_{2} y+\left(a_{1}+b_{1} y\right) w\right]^{2} \eta(y)^{2}+\left[\int_{0}^{y}\left[\rho(\tau) g_{x}-4\left(a_{1}+b_{1} \tau\right) \eta(\tau)\right] d \tau\right]^{2}
\end{aligned}
$$




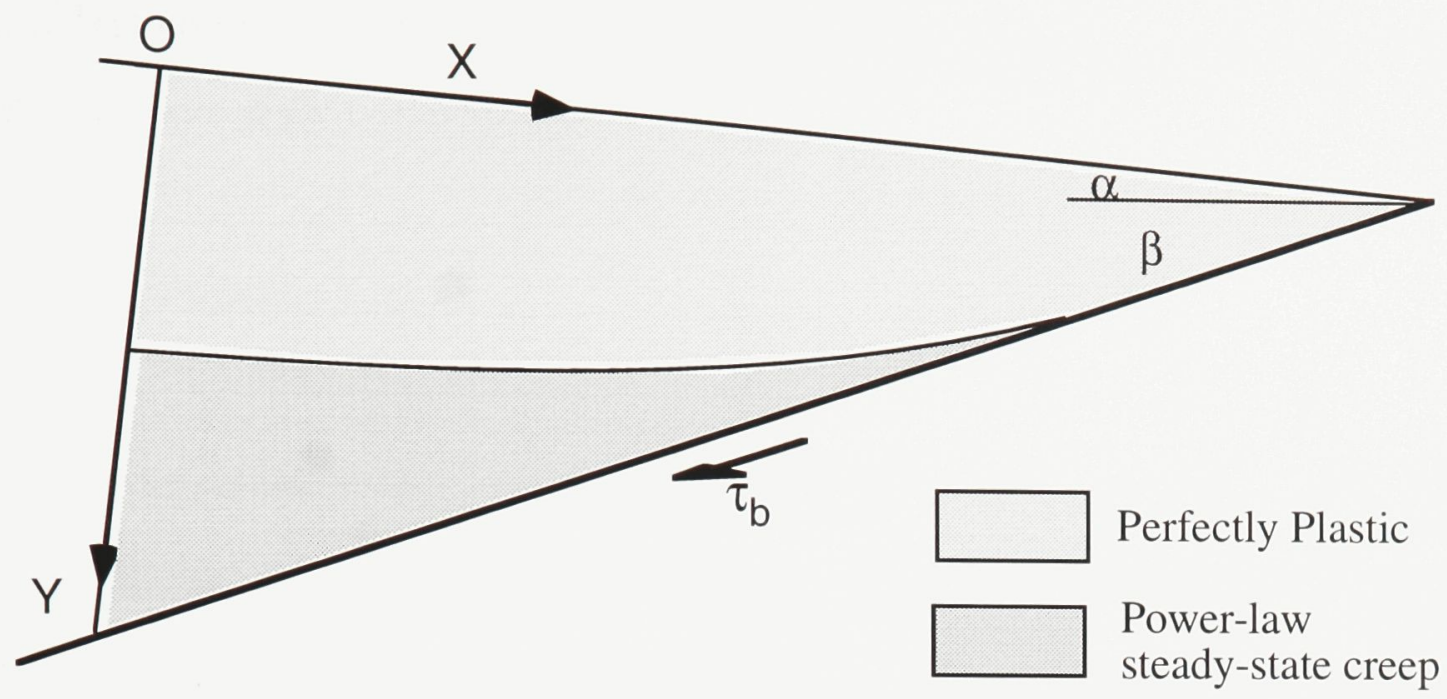

Figure 6.5 An orogenic wedge subdivided into upper perfectly plastic and lower power-law creep layers.

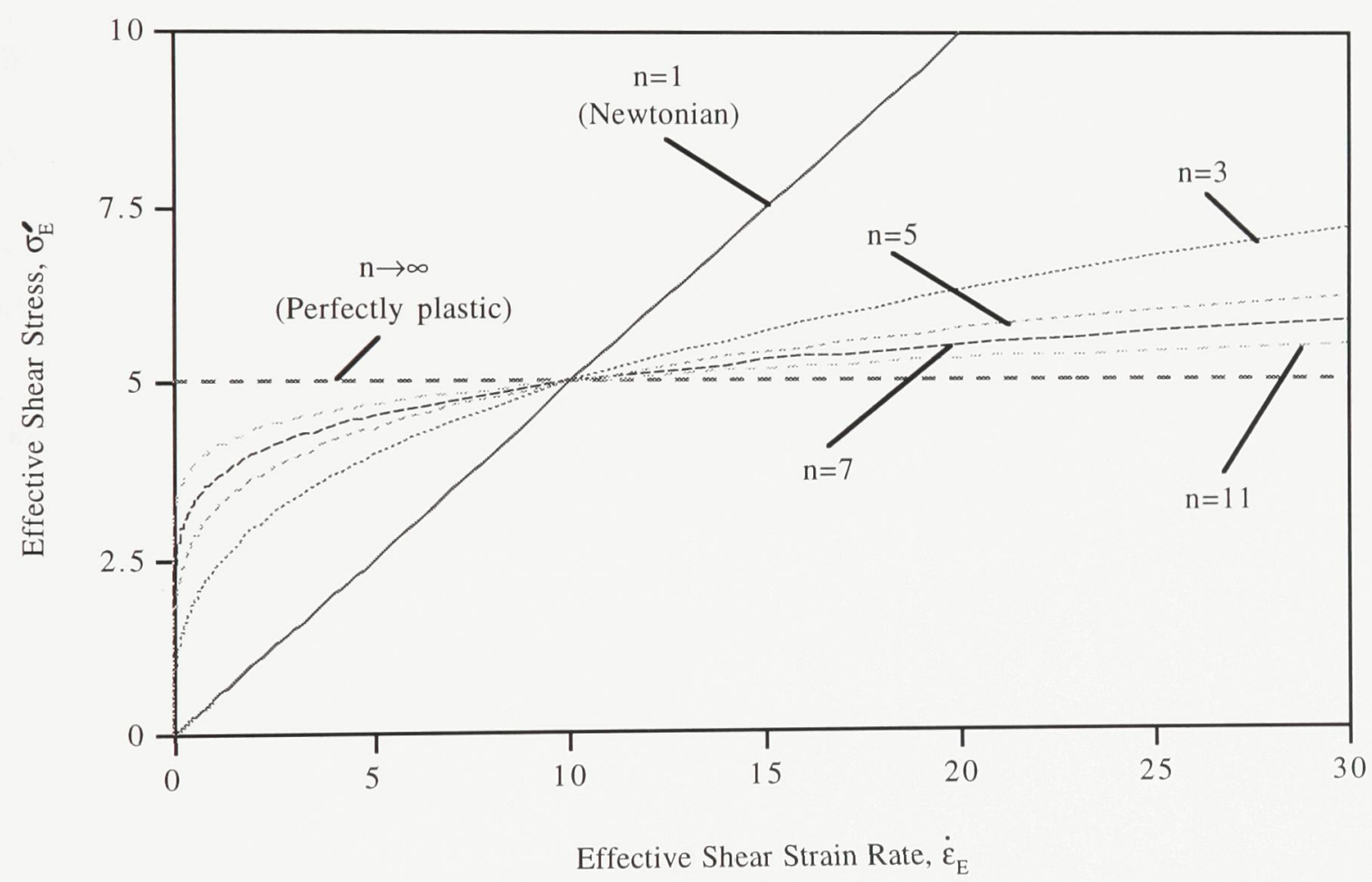

Figure 6.6 A general power-law creep model $\left(\dot{\varepsilon}_{E}=\lambda \sigma_{E}^{\prime n}\right)$ with variable stress exponent $n$ and corresponding stress-strain rate graphs. The parameter $\lambda$ is adjusted to give the same strain rate for each curve at a stress of 5 (values of stress and strain-rate are arbitrary). 
As a first approximation, a linear function of depth $y$ has been assumed for the plastic strength function $s(y)$

$$
s(y)=s_{0}+k \rho(y) g y \cos \alpha
$$

where $s_{0}$ is the plastic strength at the top of each wedge segment and $\rho(y)$ is the density; $g$ is gravitational acceleration and $\alpha$ the topographic slope. A value of 0.1 has been applied to the constant $k$ to specify the slope at which plastic strength increases with depth. This is less than the rate of increase of frictional strength at low pressure in dry rocks, but this rates decreases with increasing pressure and pore fluid pressure ( $c f$. Ranalli, 1995).

Within each wedge segment, the top plastic strength $s_{0}$ is required to meet two conditions:

(1) The computed effective viscosity distribution $\eta(y)$ must be continuous at the depth $h_{p}$, the thickness of the top plastic layer of the concerned wedge segment (determination of $h_{p}$ will be briefly discussed at the end of this section). This continuity requirement can be easily derived from equations (5.34) and (6.5) as

$$
\frac{1}{2 \eta\left(h_{p}\right)}=\tilde{A}\left(h_{p}\right)\left[s_{0}+k \rho\left(h_{p}\right) g h_{p} \cos \alpha\right]^{\frac{\tilde{n}\left(h_{p}\right)-1}{2}}
$$

where the functions $\tilde{A}(y)$ and $\tilde{n}(y)$ have been defined in the context of equation (5.34).

(2) The computed effective viscosity distribution $\eta(y)$ must satisfy the basal shear stress boundary conditions (see equation 5.32 and Figure 5.4).

In other words, for a given wedge segment with upper plastic and lower power-law layers, the effective viscosity $\eta(y)$ is determined by the following steps: firstly, assign 
initial values to both parameters $s_{0}$ and $\gamma$ (see equation 5.33); secondly, adjust $s_{0}$ to satisfy the following two equations

$$
\begin{array}{ll}
\sigma_{E}^{\prime 2}=\left[s_{0}+k \rho(y) g y \cos \alpha\right]^{2} & 0 \leq y \leq h_{p} \\
\frac{1}{2 \eta(y)}=\tilde{A}(y) \sigma_{E}^{\prime \frac{\tilde{n}(y)-1}{2}} & h_{p} \leq y \leq \bar{h}
\end{array}
$$

where $\bar{h}$ is thickness of the wedge segment and $\sigma_{E}^{\prime}$ the effective shear stress; thirdly, check if the resulting distribution of $\eta(y)$ is in agreement with the basal shear stress boundary conditions (i.e., equation 5.32). This three-step procedure is repeated until two values for $s_{0}$ and $\gamma$ are found that satisfy all the constraints.

For those wedge segments (such as in the thin-skinned part of the wedge) where the temperature throughout the whole depth is below the critical temperature (and therefore $h_{p} \geq \bar{h}$ ), plastic flow is the only deformation pattern. In this case only the first and the third steps are required to take to compute the acceptable values for parameters $s_{0}$ and $\gamma$.

The process of calculating $s_{0}$ and $\gamma$ described above is a two point boundary value problem and can be solved by several numerical techniques (for detailed discussion see Press et al. , 1992). One of these techniques, the so-called shooting method is adopted in this thesis.

The thickness of the top plastic layer, $h_{p}$, depends on both the geothermal gradient and the material composition of the wedge. The critical temperature $T_{c}(y)$ is about one half of $T_{m}(y)$, which is here assumed constant for a given material (cf. subsection 6.4.1). Comparison between $T_{c}(y)$ and the temperature distribution $T(y)$ gives $h_{p}$ (Figure 6.7). 


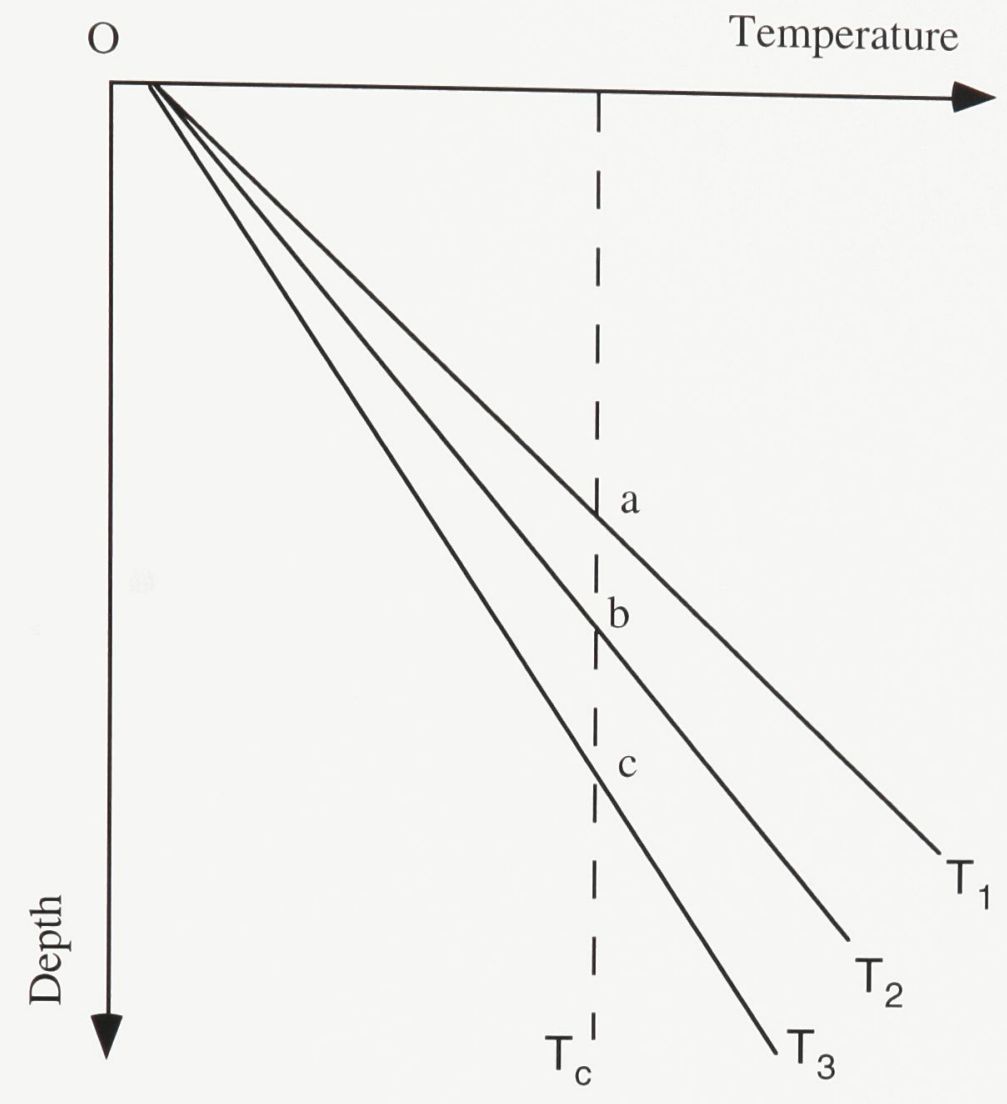

Figure 6.7 Determination of the thickness of the top plastic layer. $T_{c}$ is the depth distribution of the critical plastic temperature, assuming uniform composition; $T_{1}, T_{2}$, and $T_{3}$ are three geotherms with different geothermal gradients. Depths at points $a, b$, and $c$ are the thickness of the top plastic layers for these three geotherms. 


\subsubsection{Effects of model parameters on the dynamics of orogenic wedges with plastic upper layer}

The model parameters investigated in this section include (a) upper and lower slopes of the wedge (i.e., $\alpha$ and $\beta$ ); (b) material composition and dry/wet conditions; (c) temperature; (d) density of the wedge material, and (e) basal shear resistance. A temperature of $0{ }^{\circ} \mathrm{C}(\approx 273 \mathrm{~K})$ on the top of the wedge is used in all computations.

Boundary conditions for all models in this chapter are specified in a way identical to that used in Section 5.5 (i.e., equations $5.38 \mathrm{a}-\mathrm{d}$ ). The variation of density of the wedge material is parameterized by the following linear function of depth

$$
\rho=\rho_{0}+\rho_{1} y
$$

where $\rho_{0}$ and $\rho_{1}$ are constants. (In most calculations, the density has been taken constant for simplicity, but this does not entail any loss of generality.)

Four cases with associated model parameters are listed in Table 6.3. The top velocity boundary conditions are specified such that the longitudinal strain rate along the top of the wedge varies linearly in the $x$-direction from $-10^{-15} \mathrm{~s}^{-1}$ at the rear to $-10^{-14} \mathrm{~s}^{-1}$ at the front of the wedge. It should be noted that, during the discussion, reference will be made to some cases not listed in Table 6.3.

Case A2 (Figures 6.8) serves as a reference case with the typical power-law rheology of anorthosite and a low geothermal gradient. The thickness of the top plastic layer is about $28.5 \mathrm{~km}$ as represented by the dashed line in the velocity field diagram (Figure 6.8a). The wedge material moves in a pattern roughly comparable to the flow of a fluid jetting out a diverging channel. The maximum shear stress trajectories (Figure 6.8b) 
Table 6.3 Parameters for viscoplastic wedges with $L=200 \mathrm{~km}$ and top shortening rate increasing towards the front.

\begin{tabular}{ccccc}
\hline & \multicolumn{5}{c}{ Case $^{*}$} \\
\cline { 2 - 5 } Parameter & $\mathrm{A} 2$ & $\mathrm{~B} 2$ & $\mathrm{C} 2$ & $\mathrm{D} 2$ \\
\hline$\alpha$ & $3^{\circ}$ & $3^{\circ}$ & $3^{\circ}$ & $3^{\circ}$ \\
$\beta$ & $6^{\circ}$ & $6^{\circ}$ & $6^{\circ}$ & $6^{\circ}$ \\
$u_{0}\left(\times 10^{-10} \mathrm{~m} \mathrm{~s}^{-1}\right)$ & 0 & 0 & 0 & 0 \\
$u_{1}\left(\times 10^{-15} \mathrm{~s}^{-1}\right)$ & -1 & -1 & -1 & -1 \\
$u_{2}\left(\times 10^{-20} \mathrm{~m}^{-1} \mathrm{~s}^{-1}\right)$ & -2.25 & -2.25 & -2.25 & -2.25 \\
$v_{0}\left(\times 10^{-10} \mathrm{~m} \mathrm{~s}^{-1}\right)$ & -0.4 & -0.4 & -0.4 & -0.4 \\
$v_{1}\left(\times 10^{-15} \mathrm{~s}^{-1}\right)$ & 0.2 & 0.2 & 0.2 & 0.2 \\
$\tau_{0}\left(\mathrm{MPa}^{\circ}\right.$ & -80 & -80 & -80 & -160 \\
$\tau_{1}\left(\mathrm{MPa} \mathrm{km}^{-1}\right)$ & 0.35 & 0.35 & 0.35 & 0.75 \\
$T_{0}\left(\mathrm{~K}^{\circ}\right)$ & 273 & 273 & 273 & 273 \\
$T_{1}\left(\mathrm{~K} \mathrm{~km}^{-1}\right)$ & 15 & 15 & 25 & 15 \\
$\rho_{0}\left(\mathrm{~kg} \mathrm{~m}^{-3}\right)$ & $2700^{\S}$ & 2700 & $2700^{\S}$ & $2700^{\S}$ \\
$\rho_{1}\left(\mathrm{~kg} \mathrm{~m}^{-3} \mathrm{~km}^{-1}\right)$ & 0 & 0 & 0 & 0
\end{tabular}

* Cases A2, B2, C2, and D2 refer to Figures 6.8, 6.9, 6.10, and 6.11.

A2: Power-law of anorthosite, low thermal gradient and small basal shear stress

B2: Power-law of quartzite, low thermal gradient and small basal shear stress

C2: Power-law of anorthosite, high thermal gradient and small basal shear stress

D2: Power-law of anorthosite, low thermal gradient and large basal shear stress

$\S$ a constant density is assumed for both anorthosite and quartzite given the trivial effect of variations of density on the dynamics of orogenic wedges

$\dagger$ rheological parameters for anorthosite: $A_{0}=2.06 \times 10^{-23}\left(\mathrm{~Pa}^{-3.2} \mathrm{~s}^{-1}\right), n=3.2, Q=238\left(\mathrm{~kJ} \mathrm{~mol}^{-1}\right)$

$\ddagger \quad$ rheological parameters for quartzite: $A_{0}=2.512 \times 10^{-20}\left(\mathrm{~Pa}^{-2.4} \mathrm{~s}^{-1}\right), n=2.4, Q=156\left(\mathrm{~kJ} \mathrm{~mol}^{-1}\right)$

indicate a homogenous thrust deformation regime throughout the wedge. The effective shear strain rate $\dot{\varepsilon}_{E}^{\prime}$ (Figure 6.8c) varies mainly in the longitudinal direction within the upper plastic layer, increasing from $10^{-15} \mathrm{~s}^{-1}$ at the rear to $10^{-14} \mathrm{~s}^{-1}$ at the front of the wedge; $\dot{\varepsilon}_{E}^{\prime}$ becomes a increasing function of the depth only within the rear lower powerlaw region of the wedge. The effective shear stress $\sigma_{E}^{\prime}$ (Figure 6.8d) varies mainly in the $y$-direction, firstly increasing with depth within the upper plastic layer and then 
decreasing with depth in the lower power-law region (Note that the small bump in the effective shear stress contour map when the power-law creep zone appears is basically an artifact of calculations, due to the iteration being stopped to speed up the computation process. Similar observations also apply to the effective shear stress contour maps for all the following cases.) The effective viscosity (Figure 6.8e) varies in both the $x$ - and the $y$ directions in the top plastic layer, increasing with depth and decreasing from the rear towards the front of the wedge; within the rear lower power-law region of the wedge the effective viscosity decreases rapidly with depth. (Note that the effective viscosity in the plastic zone of a viscoplastic wedge can be understood, based on its formal definition, as the ratio between the deviatoric stress and the corresponding strain rate which is a result of the assigned boundary conditions.) The nongravitational normal compressive stress $F(y)$ at the rear of the wedge responsible for the deformation regime (Figure 6.8f) first increases in magnitude from $-45 \mathrm{MPa}$ on the top to $-160 \mathrm{MPa}$ at the bottom of the top plastic layer and then decreases rapidly at the bottom of the wedge. The nongravitational shear stress $G(y)$ at the rear of the wedge does not show sharp changes.

To study the effect of top and basal slopes on the dynamics of wedges, a separate case was designed (not listed in Table 6.3). All model parameters are identical to those of case A1 except that its top and basal slopes are $\alpha=\beta=4.5^{\circ}$. Comparison of solutions between these two cases reveals a great deal of similarity in terms of deformation and longitudinal strain rate. The major difference is observed in the nongravitational normal stress required to maintain the deformation regime at the rear of the wedge: the magnitude of $F(y)$ in the new case is about 3-5 MPa less than that of case A2.

Wedges with variable density have also been considered, with a density of $2700 \mathrm{~kg} \mathrm{~m}^{-3}$ at the top linearly increasing to $2900 \mathrm{~kg} \mathrm{~m}^{-3}$ at the depth of $30 \mathrm{~km}^{\text {(not listed }}$ 


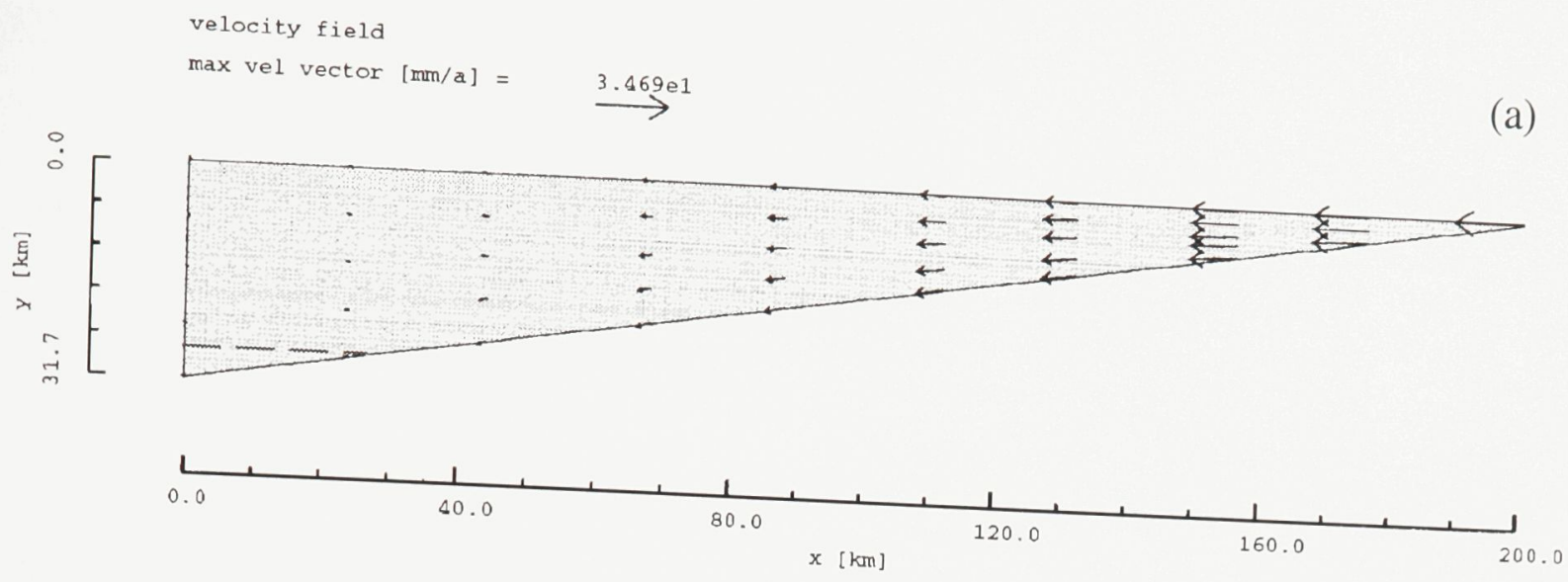

Potential Faulting Trajectories

Internal Friction Angle [deg] $=45$

(b)

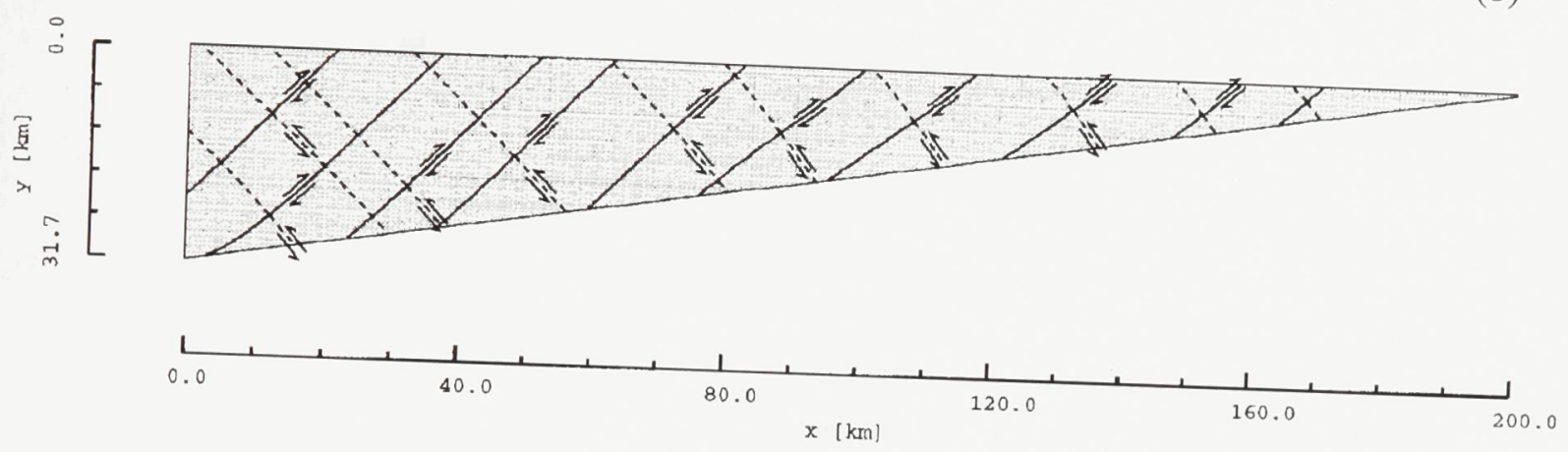

second invar. strain rate, $[1 / \mathrm{s}]$

(c)
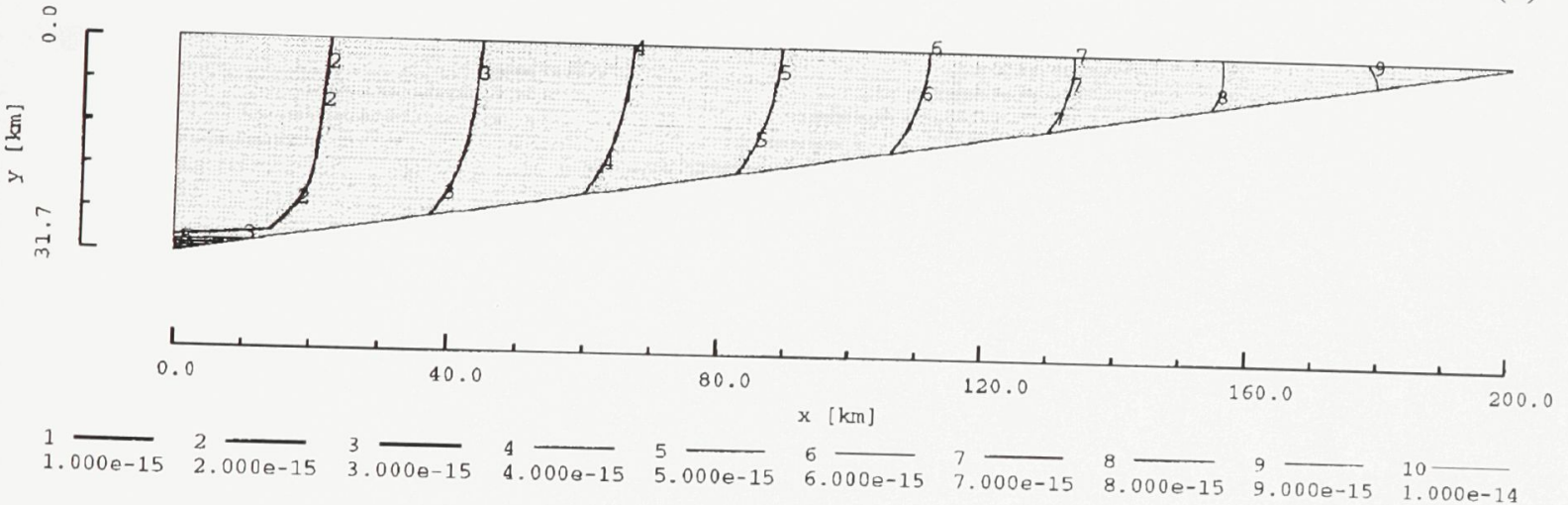

Figure 6.8 Stress and deformation within the wedge for case A2 of Table 6.3. (a) Velocity field; (b) maximum shear stress trajectories; (c) second invariant of strain rate tensor; 

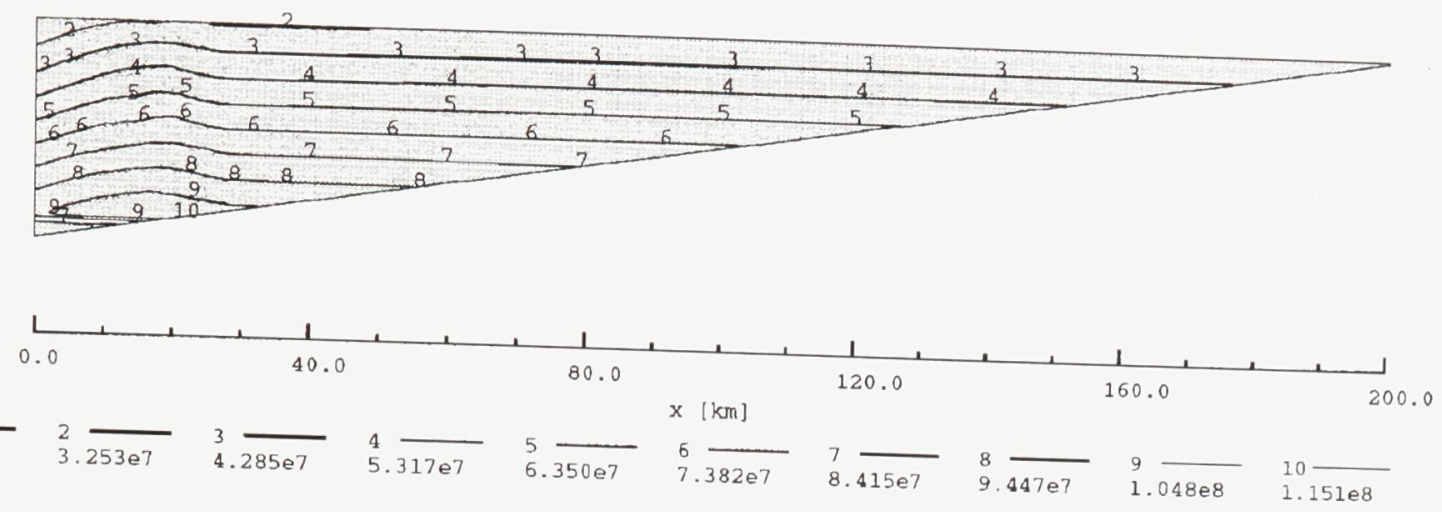

Log $10[$ eff viscosity] [Pa s]
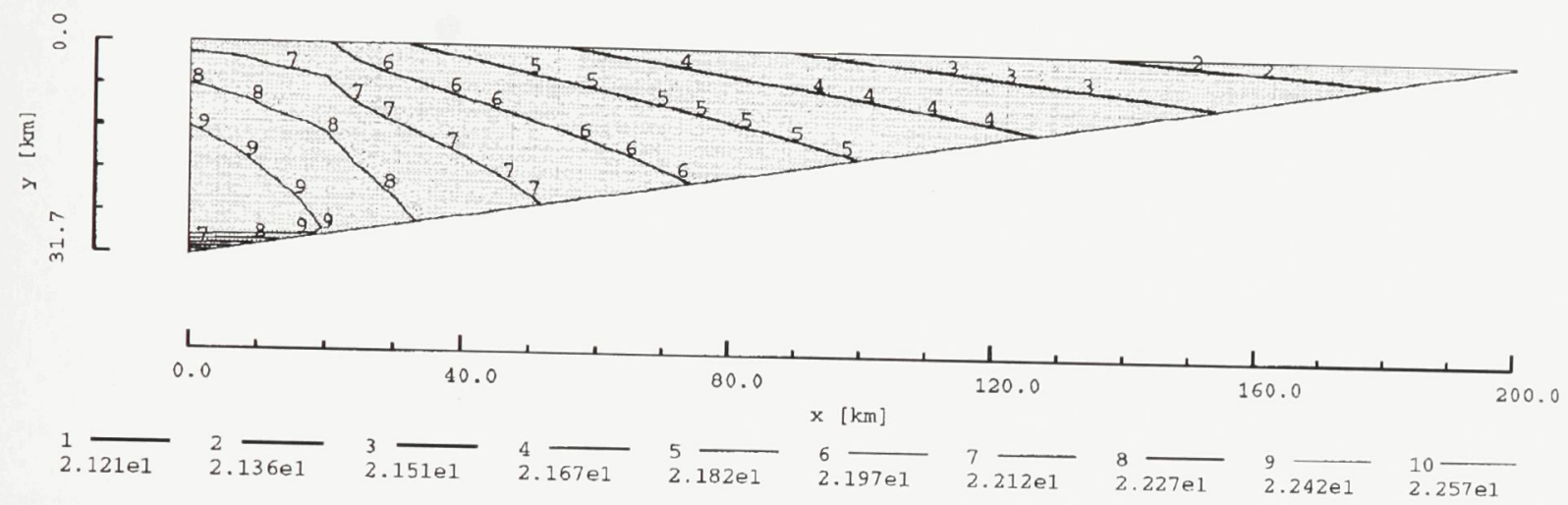

Figure 6.8

(d) second invariant of deviatoric stress tensor; (e) effective viscosity;

(continued) 


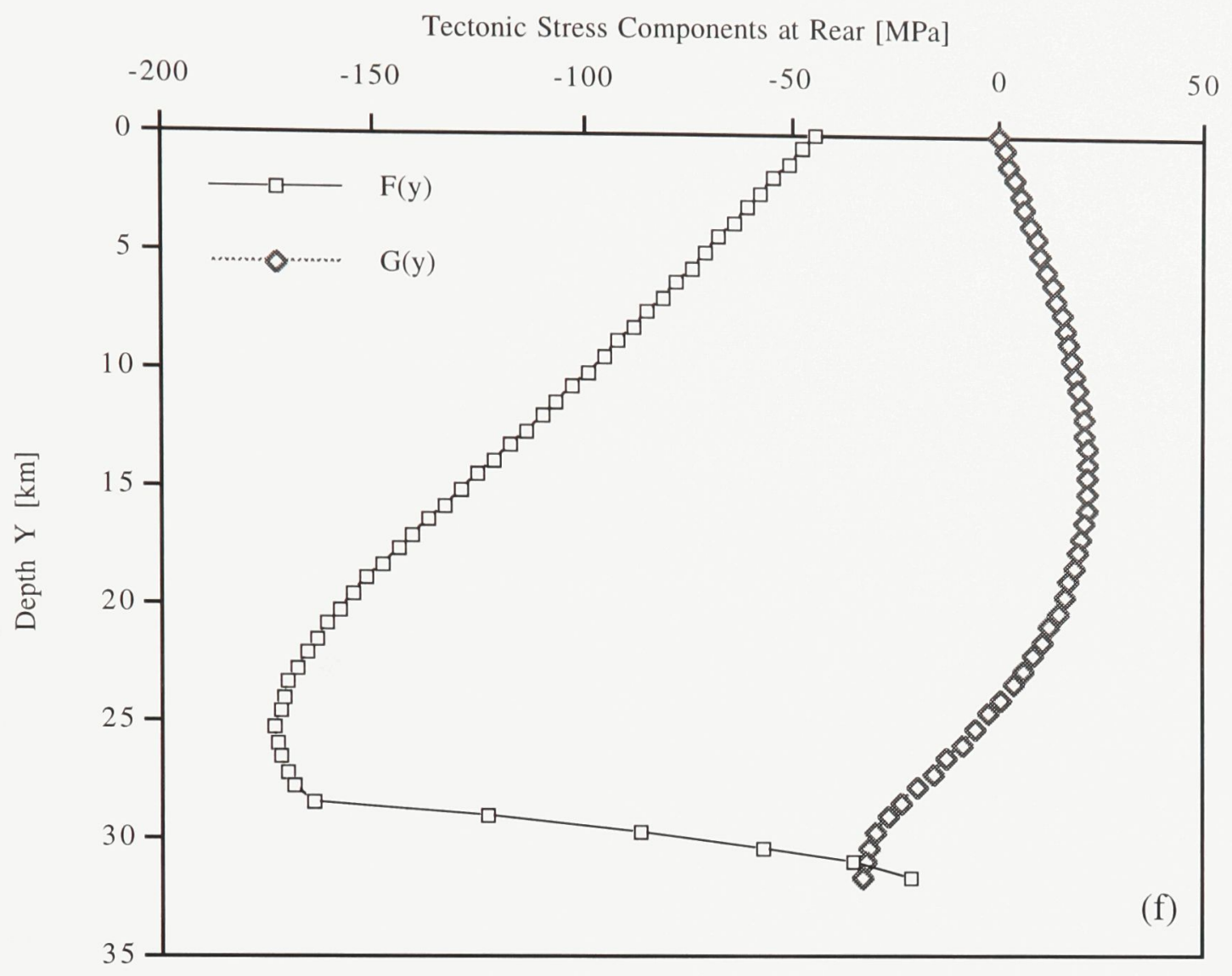

Figure 6.8 (f) tectonic-induced normal and shear stresses along the rear boundary of the wedge necessary to maintain the deformation. 
in Table 6.3). Compared to case A2, the only significant effect of the increased density is observed in the state of stress at the rear of the wedge: for the same boundary conditions, the nongravitational normal stress required to maintain the deformation regime at the rear of the wedge with increasing material density is smaller by an amount of about $5 \mathrm{MPa}$ than that with constant material density. This also justifies our assumption of constant density independent of rock type.

The rock material (quartzite) parameterized in case B2 (Figure 6.9) is much softer than the anorthosite of case A2 (see Tables 6.1 and 6.2). Since boundary conditions for cases A2 and B2 are identical (see Table 6.3), comparison between these two cases shows the effects of rheology on the deformation and stress pattern. Most of the deformation is concentrated in the rear lower power-law region of the wedge (Figure 6.9a) where the dominant flow pattern is laminar (Figure 6.9b). The strong negative horizontal component of velocity appearing in the rear lower power-law region is a consequence of the rapid decrease in the effective viscosity combined with the basal shear stress. The spatial distribution of the effective shear strain rate $\dot{\varepsilon}_{E}^{\prime}$ (Figure 6.9c) shows a similar pattern. The effective shear stress $\sigma_{E}^{\prime}$ (Figure 6.9d) varies mainly in the $y$-direction, increasing from about $6 \mathrm{MPa}$ at the top to about $79 \mathrm{MPa}$ at the bottom of the wedge. The effective viscosity (Figure 6.9e) does not vary significantly in the top plastic layer but decreases mainly in the $y$-direction from $7.8 \times 10^{21} \mathrm{~Pa} \mathrm{~s}$ at the bottom of the top plastic layer to $1.4 \times 10^{19} \mathrm{~Pa} \mathrm{~s}$ at the bottom of the wedge. Similar to case A2 (Figure 6.8f), the nongravitational normal stress at the rear of the wedge (Figure 6.9f) first increases in magnitude from -12 $\mathrm{MPa}$ at the top to $-80 \mathrm{MPa}$ at the bottom of the top plastic layer, then decreases rapidly towards the bottom of the wedge; the nongravitational shear stress at the rear of the wedge does not show a significant change when crossing the bottom of the plastic layer. 


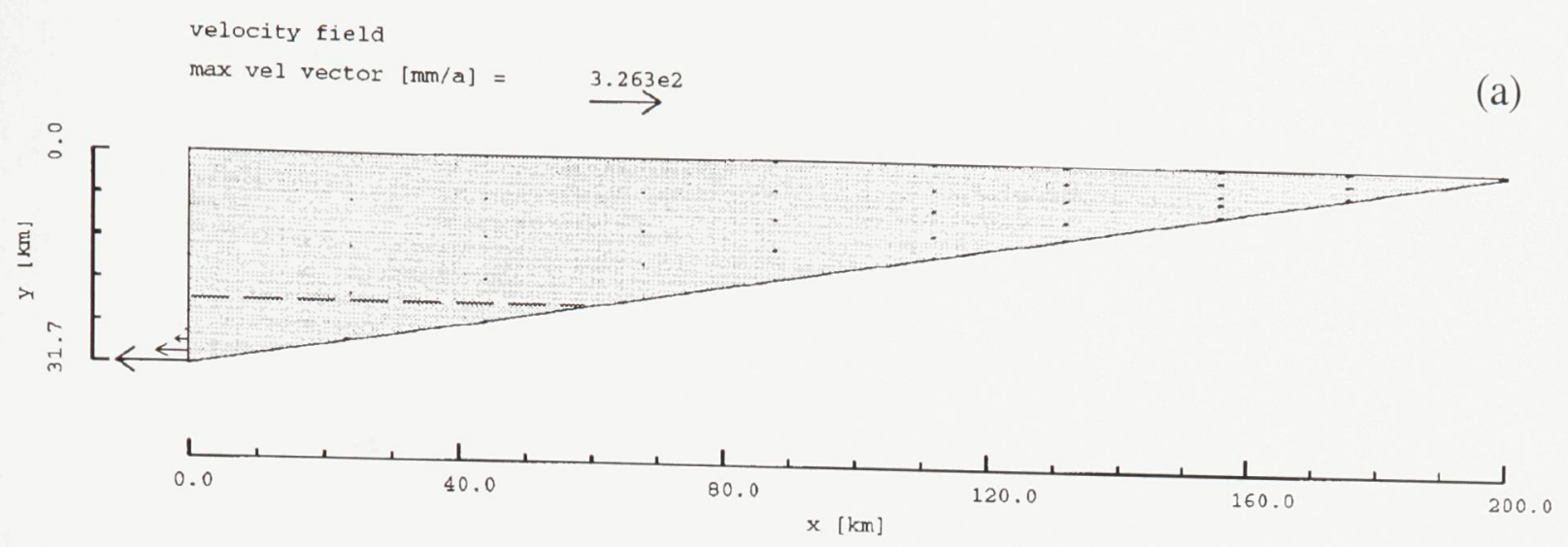

Potential Faulting Trajectories

Internal Friction Angle $[\mathrm{deg}]=45$

(b)
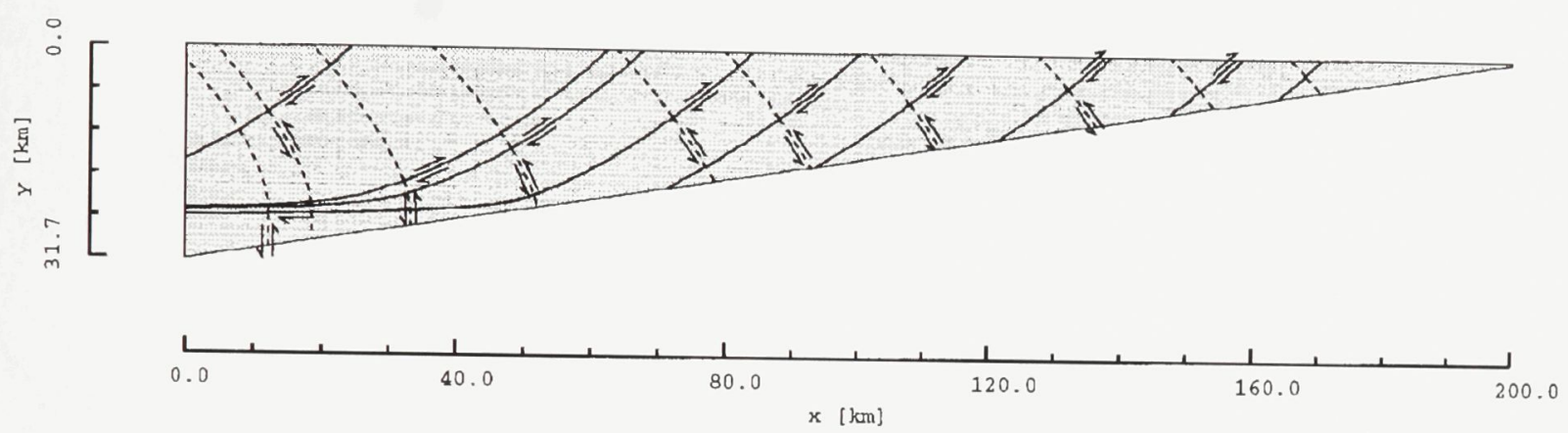

second invar. strain rate, [1/s]

(c)
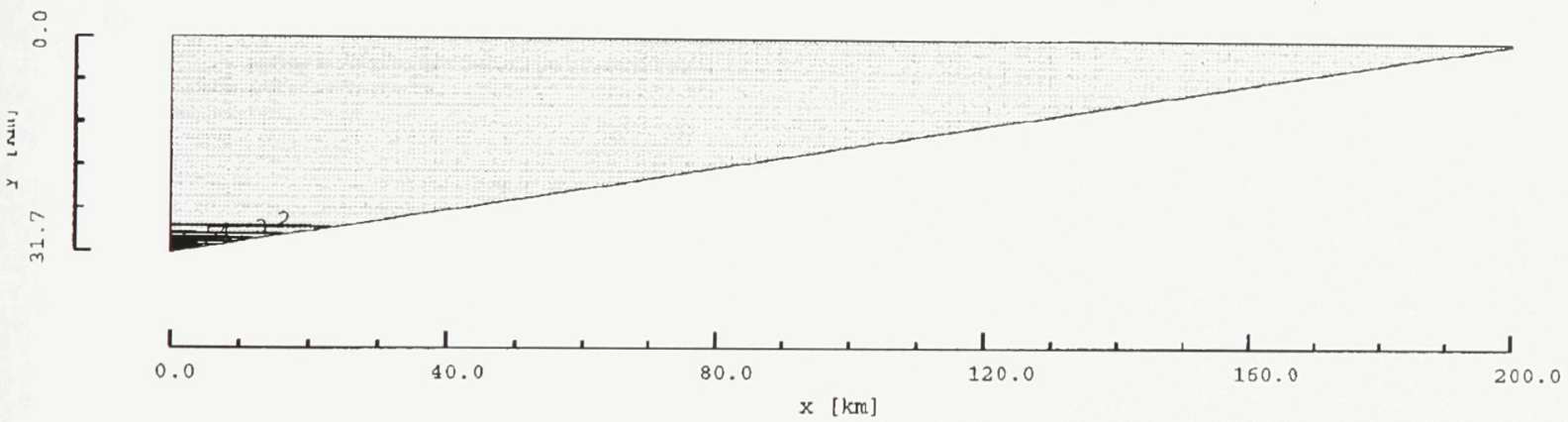

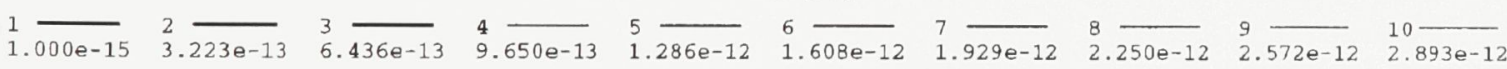

Figure 6.9 Stress and deformation within the wedge for case B2 of Table 6.3. (a) Velocity field; (b) maximum shear stress trajectories; (c) second invariant of strain rate tensor; (continued) 

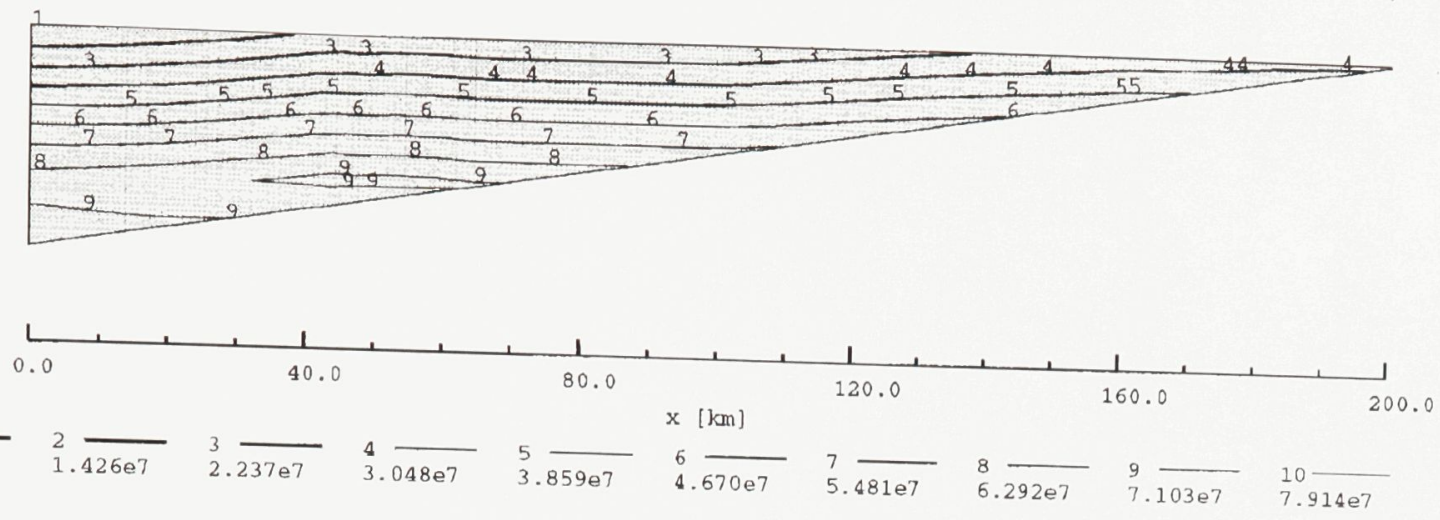

$\log 10$ [eff viscosity) [Pa s]
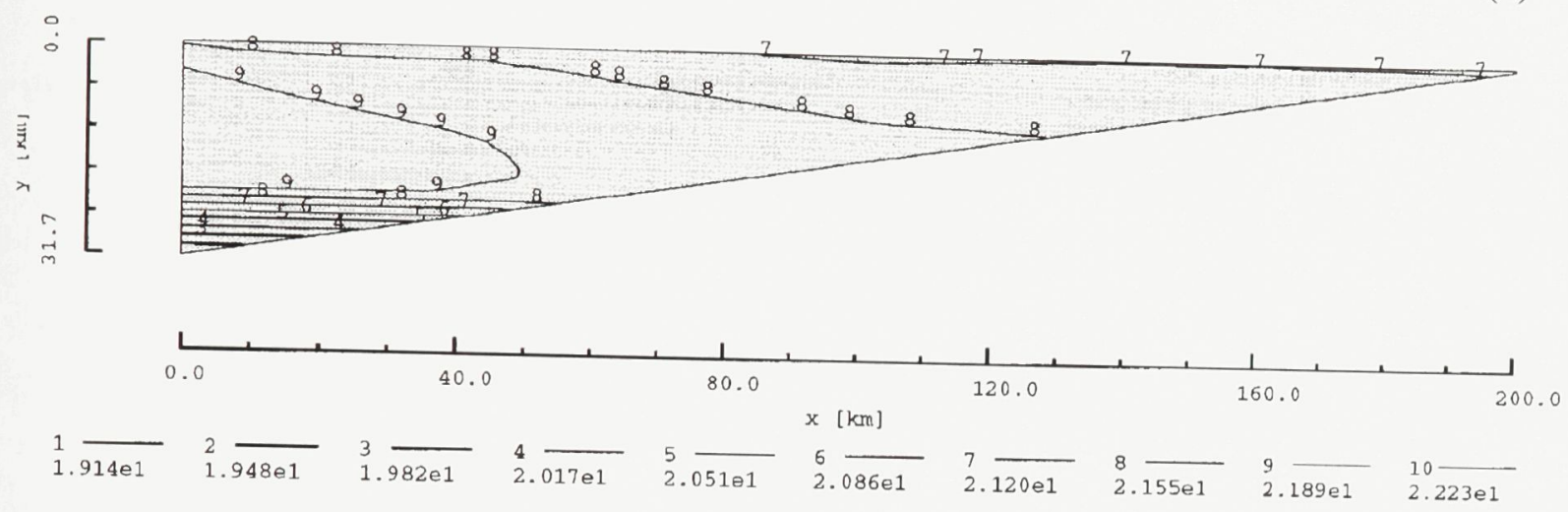

Figure 6.9

(d) second invariant of deviatoric stress tensor; (e) effective viscosity;

(continued) 


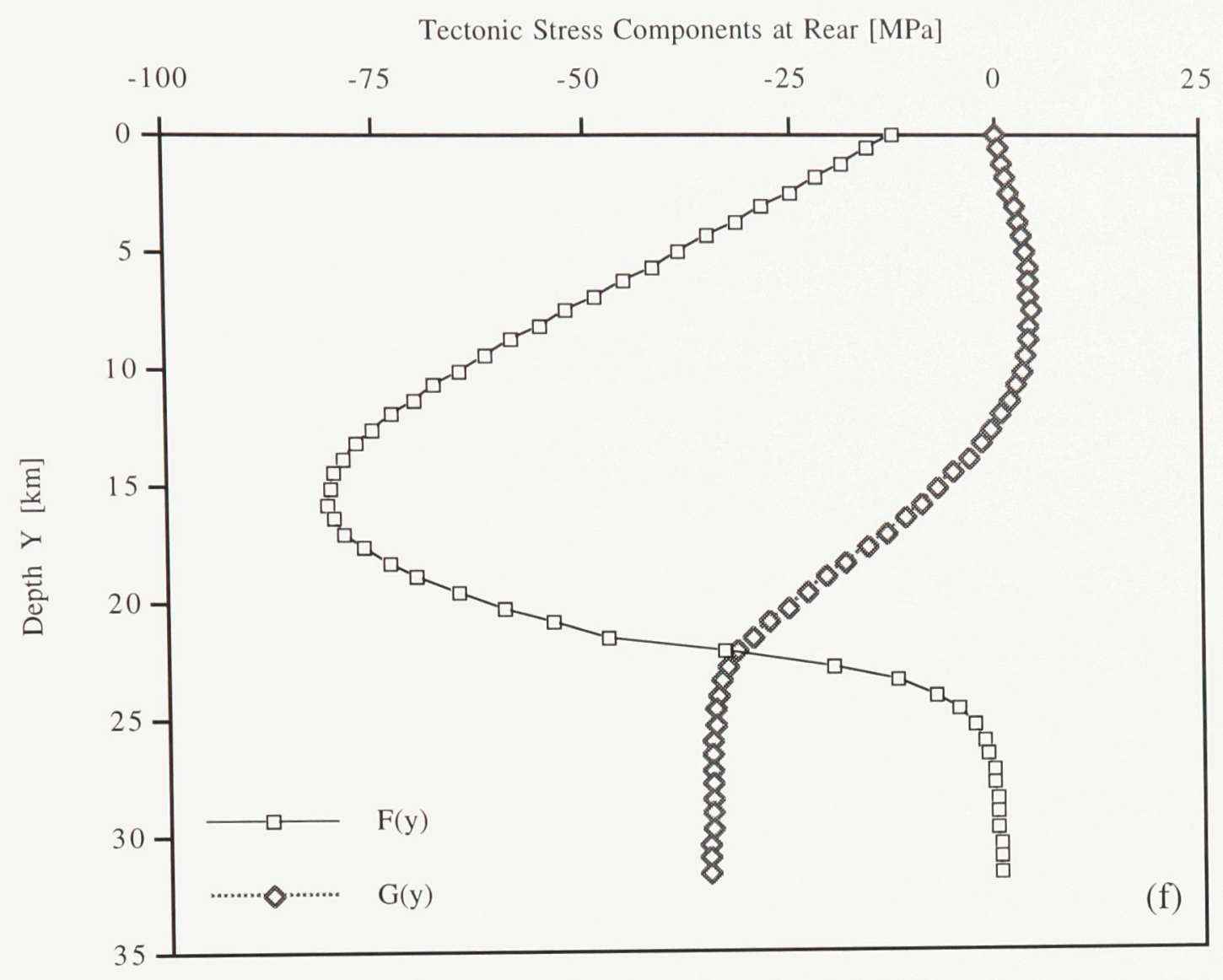

Figure 6.9 (f) tectonic-induced normal and shear stresses along the rear boundary of the wedge necessary to maintain the deformation. 
The effect of hydration within the wedge was examined by comparing case B2 (with dry quartzite material) to a case with wet quartzite material (not listed in Table 6.3). All the boundary conditions are the same for this new case and case B2. Comparison shows that (a) wedges with wet material show a larger region of predominant laminar flow; (b) the depth variation of longitudinal strain rate is larger in wet wedges than in dry wedges; and (c) the nongravitational normal stress at the rear of the wedge is also smaller for the wet than that for the dry wedge.

Results for case C2 are shown in Figure 6.10. Comparison between cases A2 and C2 shows the effect of the thermal gradient on the stress, strain rate and velocity fields within a wedge with a top plastic layer. The thickness of the plastic layer in case $\mathrm{C} 2$ (with high thermal gradient of $25 \mathrm{~K} \mathrm{~km}^{-1}$ ) is $17 \mathrm{~km}$ compared to $28.5 \mathrm{~km}$ in case A2. Similar to case B2, most of the deformation within the wedge in case $\mathrm{C} 2$ is concentrated in the rear lower power-law region (Figure 6.10a) where the predominant flow pattern is laminar (Figure 6.10b), as shown also in the spatial distribution of the effective shear strain rate $\dot{\varepsilon}_{E}^{\prime}$ (Figure 6.10c). The effective shear stress $\sigma_{E}^{\prime}$ (Figure 6.10d) varies in both the $x$-and $y$-directions in the top plastic layer, but varies mainly in the $y$-direction within the lower power-law region. The effective viscosity (Figure 6.10e) does not vary significantly in the top plastic layer, but decreases mainly in the $y$-direction in the viscous layer from $8.7 \times 10^{21} \mathrm{~Pa}$ s to $4.9 \times 10^{16} \mathrm{~Pa}$ at the bottom of the wedge. The nongravitational normal stress at the rear of the wedge (Figure 6.10f) first increases in magnitude from -20 MPa at the top to $-75 \mathrm{MPa}$ at the bottom of the top plastic layer and then decreases rapidly towards the bottom of the wedge.

The effect of temperature on the dynamics of wedge deformation is very similar to that of hydrated minerals within the wedge: both increasing temperature and $\mathrm{H}_{2} \mathrm{O}$-content make the wedge material softer. 


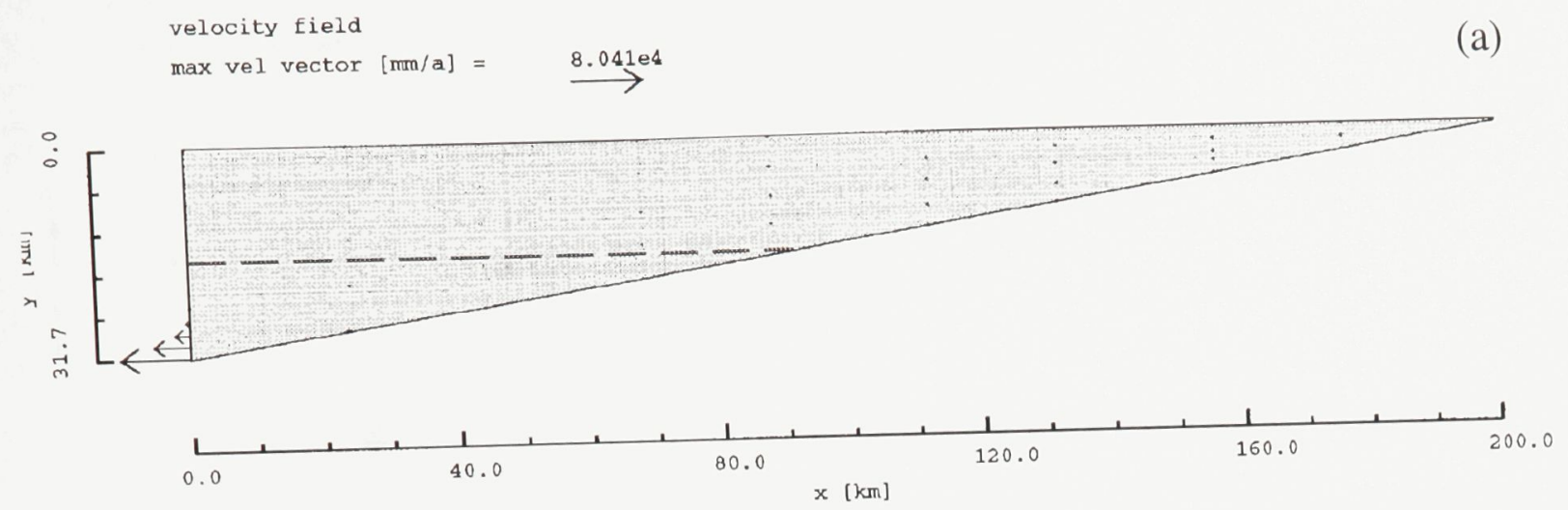

(b)

Potential Faulting Trajectories

Internal Friction Angle [deg] $=45$

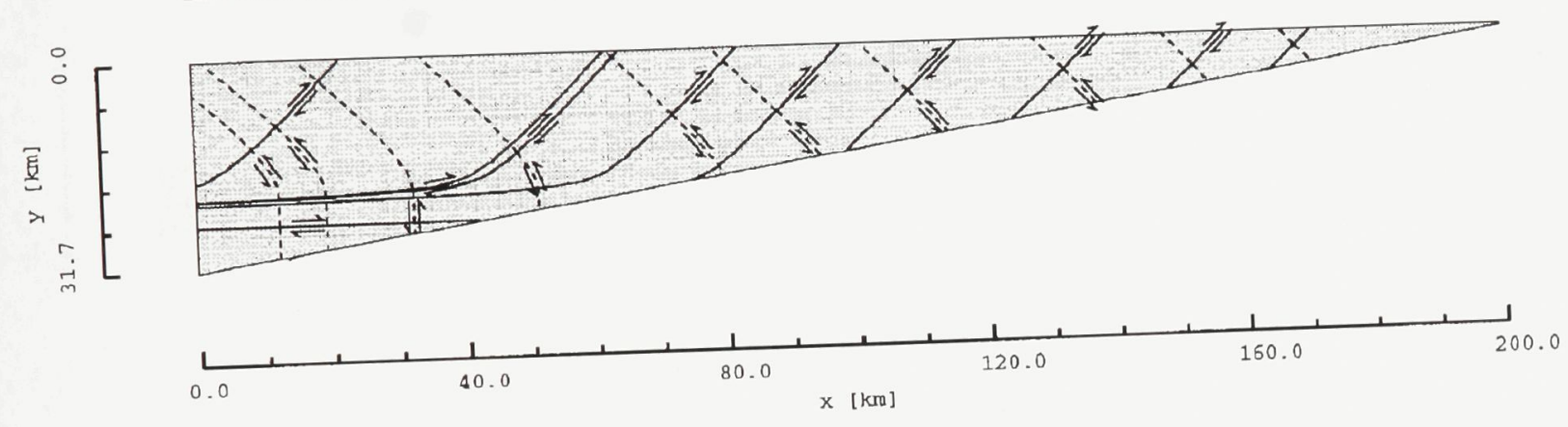

(c)

second invar. strain rate, $[1 / \mathrm{s}]$

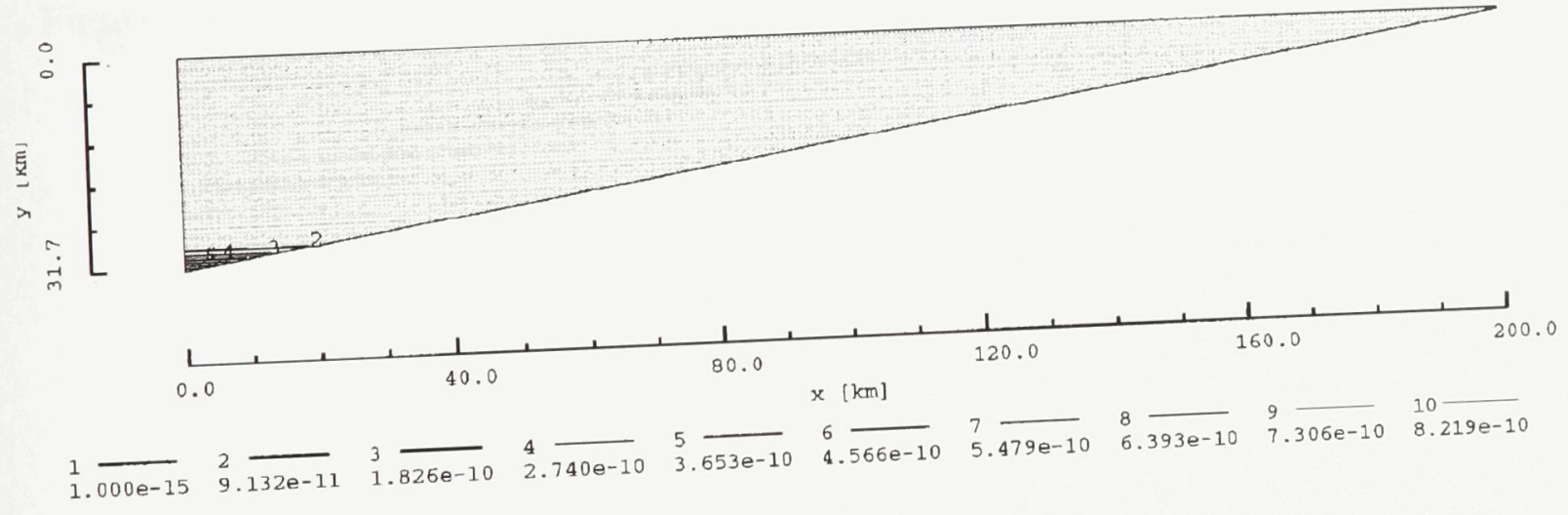

Figure 6.10 Stress and deformation within the wedge for case $\mathrm{C} 2$ of Table 6.3. (a) Velocity field; (b) maximum shear stress trajectories; (c) second invariant of strain rate tensor; 

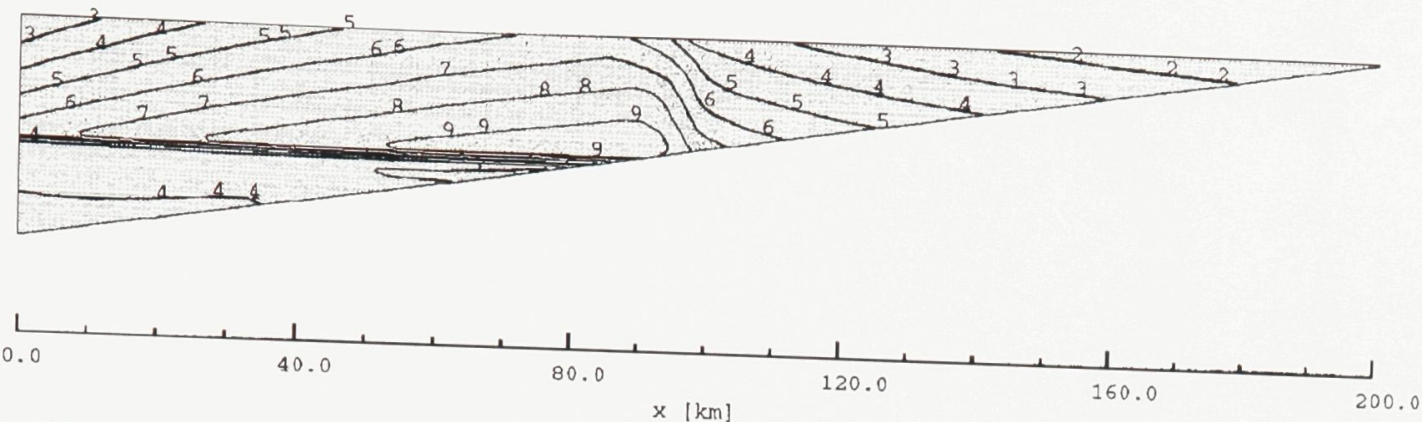

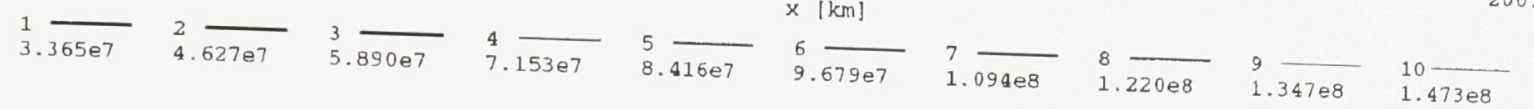

Log10[eff viscosity] [Pa s]
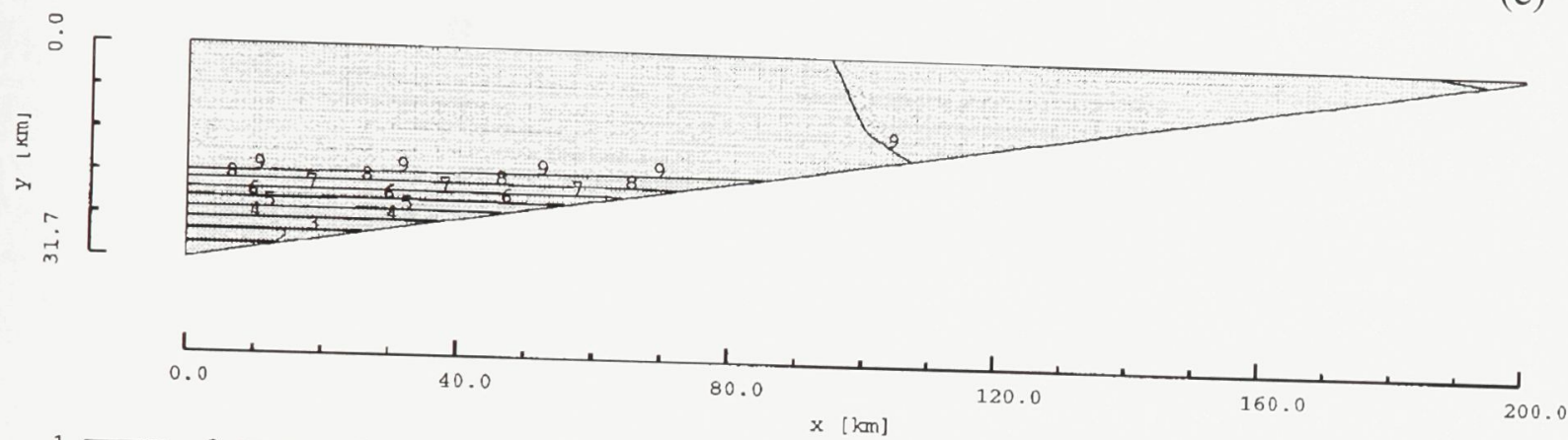

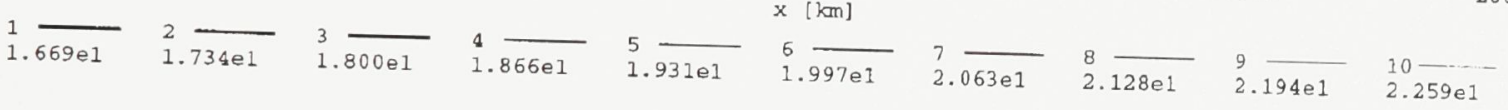

Figure 6.10

(d) second invariant of deviatoric stress tensor; (e) effective viscosity;

(continued) 


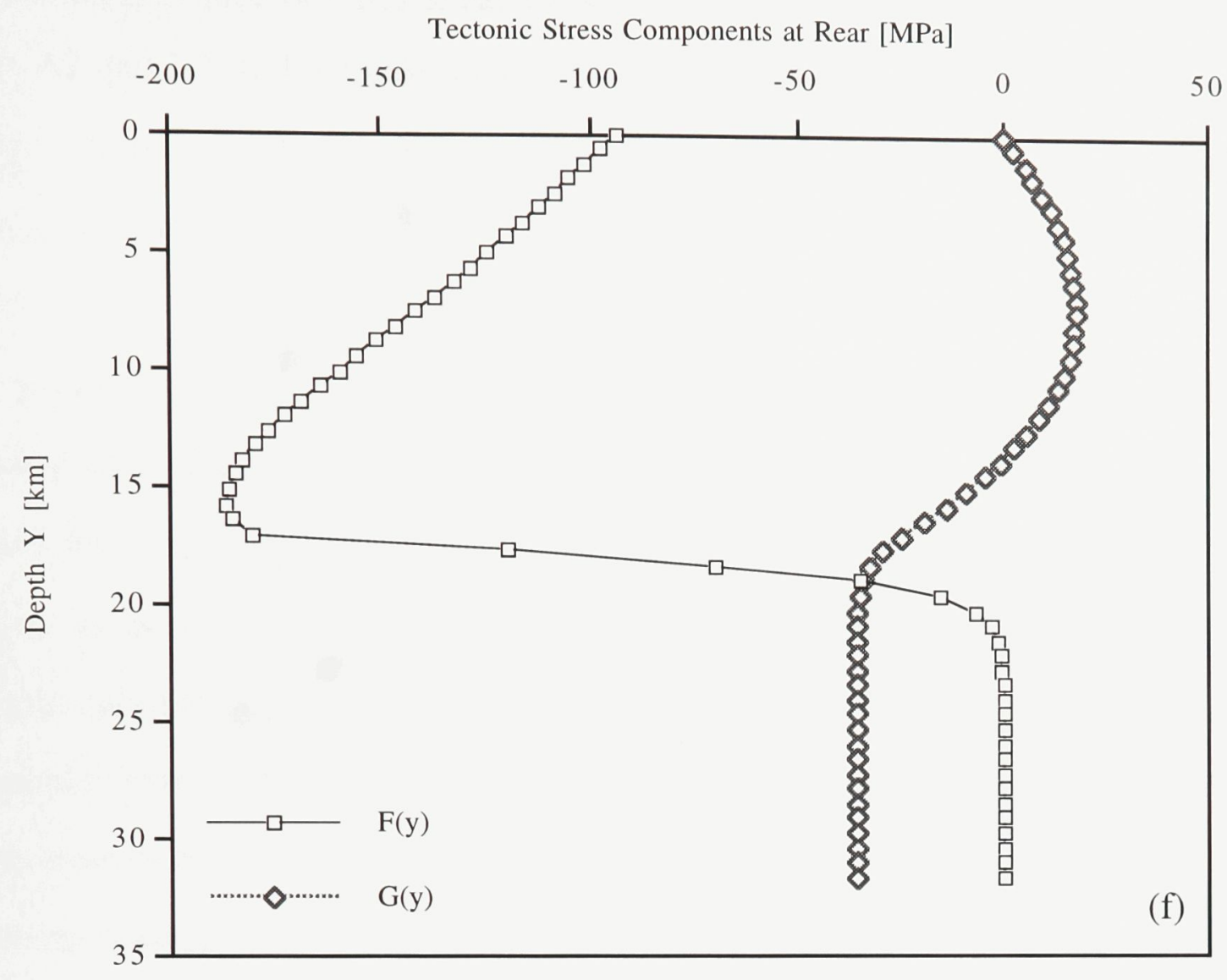

Figure 6.10 (f) tectonic-induced normal and shear stresses along the rear boundary of the wedge. 
The significance of basal shear stress was investigated by the comparison between cases A2 and D2. In both cases, the basal shear stress $\tau_{b}$ is $-10 \mathrm{MPa}$ at the front of the wedge; $\tau_{b}$ in case D2 increases linearly in magnitude to $-160 \mathrm{MPa}$ at the rear of the wedge, compared to -80 MPa in case A2. Results for case D2 are shown in Figure 6.11. A larger $\tau_{b}$ increases the shear strain rate and therefore the longitudinal flow in the lower rear power-law region of the wedge (Figure 6.11a), decreases the slopes of potential thrust planes (Figure 6.11b), and increases the effective shear strain rate $\dot{\varepsilon}_{E}^{\prime}$ (Figure $6.11 \mathrm{c}$ ) and the effective shear stress $\sigma_{E}^{\prime}$ (Figure 6.11d). The effective viscosity (Figure 6.11e), as usual, decreases significantly within the rear lower power-law region. The nongravitational normal stress at the rear of the wedge (Figure 6.11f) first increases in magnitude from $-160 \mathrm{MPa}$ on the top to $-280 \mathrm{MPa}$ and then gradually decreases towards the bottom of the wedge; the nongravitational shear stress varies nonlinearly with depth, reaching a maximum (absolute) value of about $-110 \mathrm{MPa}$ at the bottom of the wedge.

\subsubsection{Orogenic wedges with various deformation patterns}

All the cases computed in previous subsection (see Table 6.3) had the same velocity boundary condition at the top of the wedge, resulting in longitudinal compression. In this subsection, attention is focused on the effects of velocity boundary conditions: wedges in extension, wedges in compression, and wedges with frontal compression and rear extension. Three cases are studied (Table 6.4). Common assumptions are that (a) the $v$ component of the velocity on the top of the wedge decreases linearly in the $x$-direction from $-5 \mathrm{~mm} \mathrm{a}^{-1}$ (upward) at the rear to zero at the front of the wedge; (b) the basal shear stress decreases linearly with position from $-80 \mathrm{MPa}$ at the rear to zero at the front of the wedge; and (c) the density of rock material remains constant throughout the wedge with $\rho$ $=2700 \mathrm{~kg} \mathrm{~m}^{-3}$. 


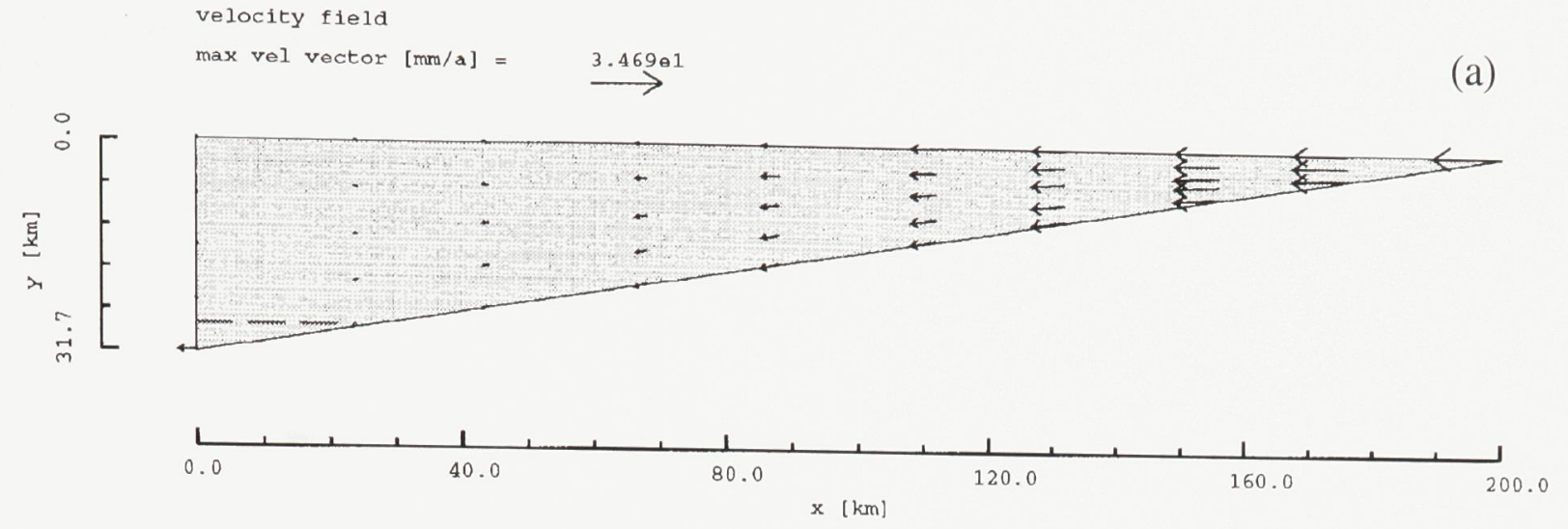

Potential Faulting Trajectories

Internal Friction Angle [deg] $=\mathbf{4 5}$

(b)
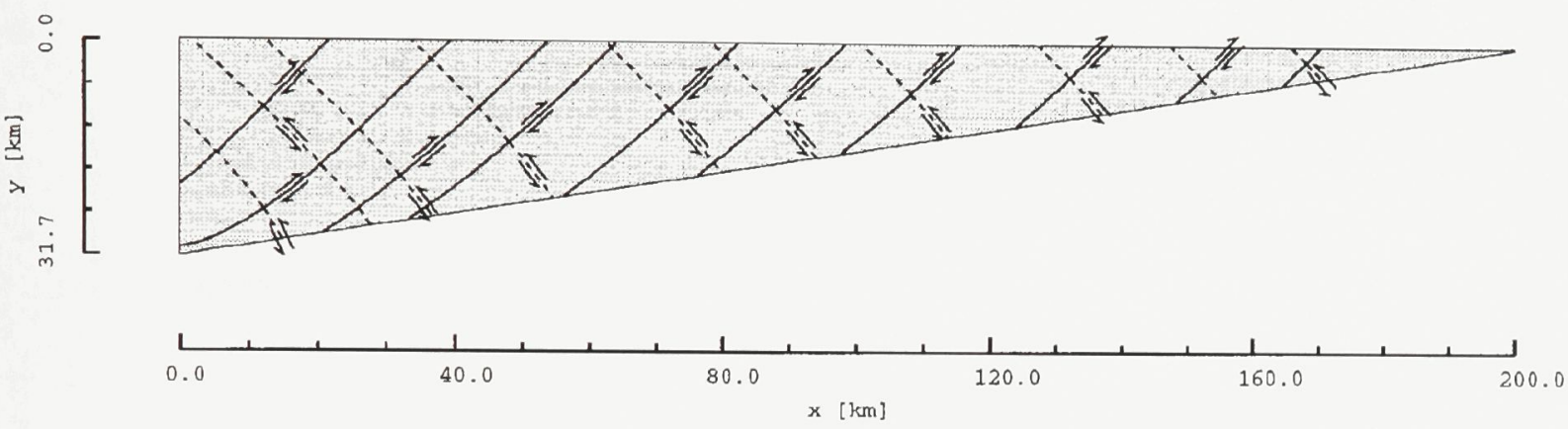

second invar. strain rate, [1/s]

(c)

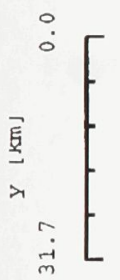
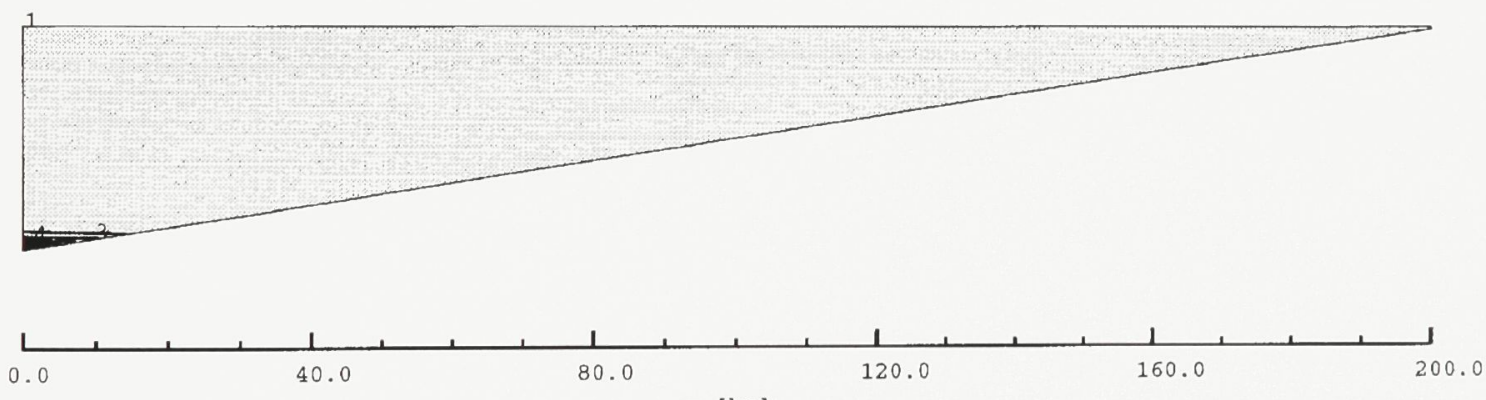

$x[\mathrm{~km}]$

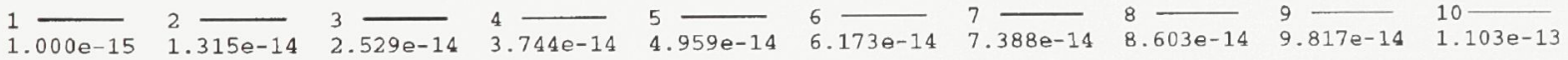

Figure 6.11 Stress and deformation within the wedge for case D2 of Table 6.3. (a) Velocity field; (b) maximum shear stress trajectories; (c) second invariant of strain rate tensor;

(continued) 

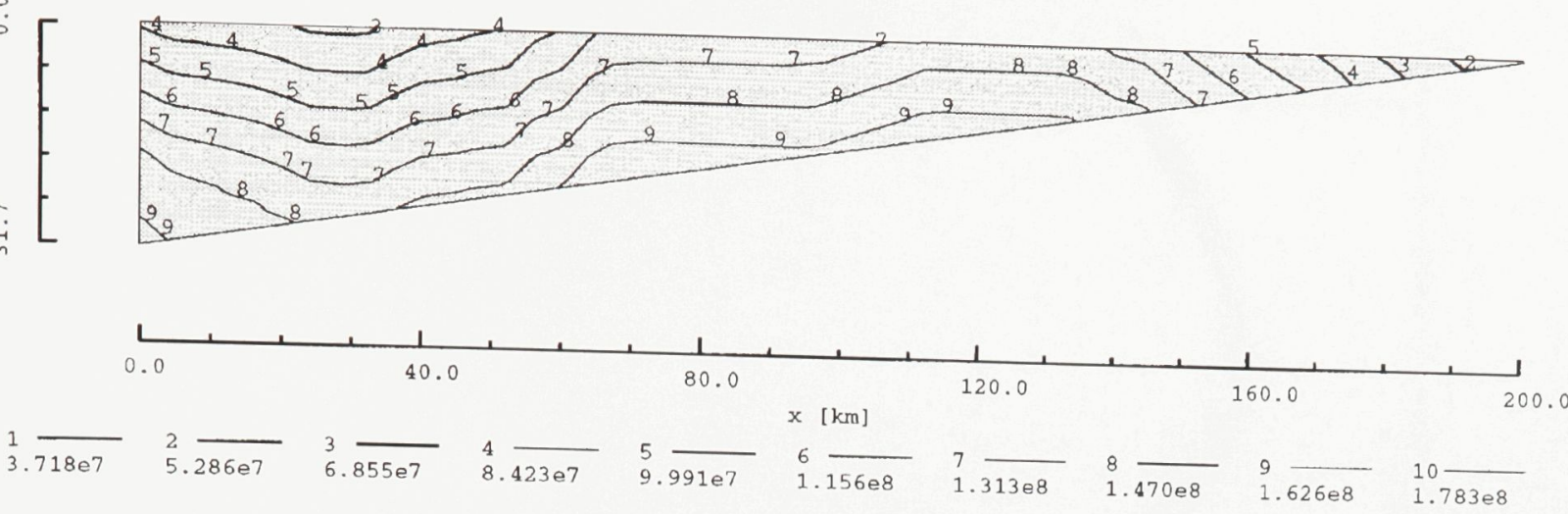

$\log 10[e f f$ viscosity] [Pa s]
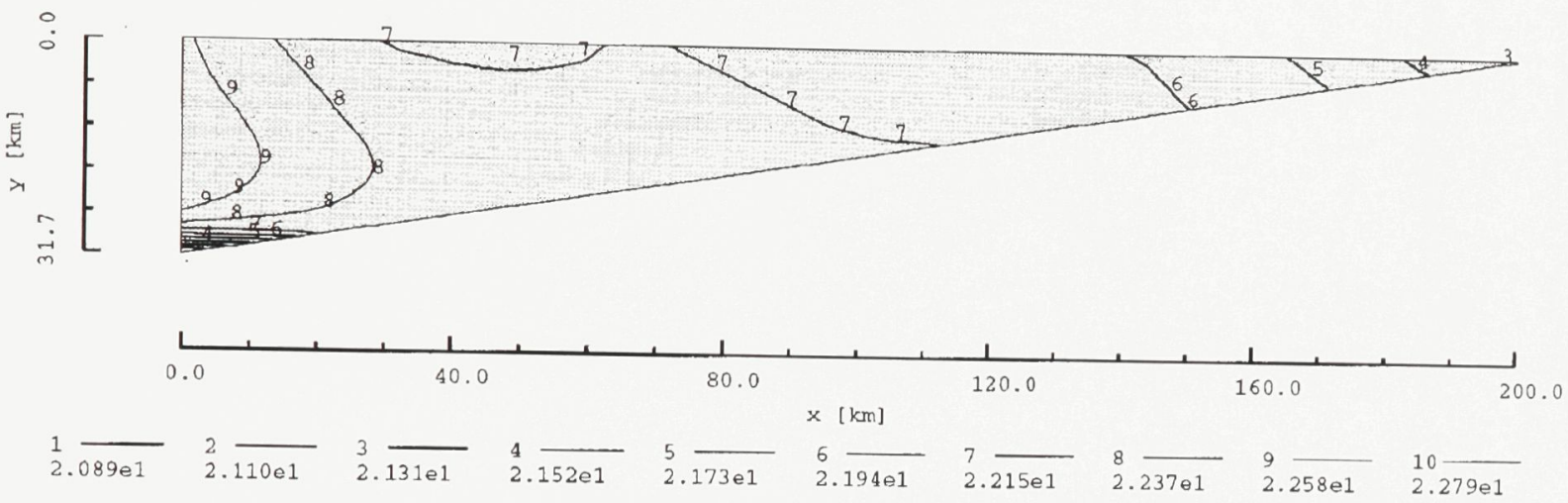

Figure 6.11 (d) second invariant of deviatoric stress tensor; (e) effective viscosity;

(continued) 


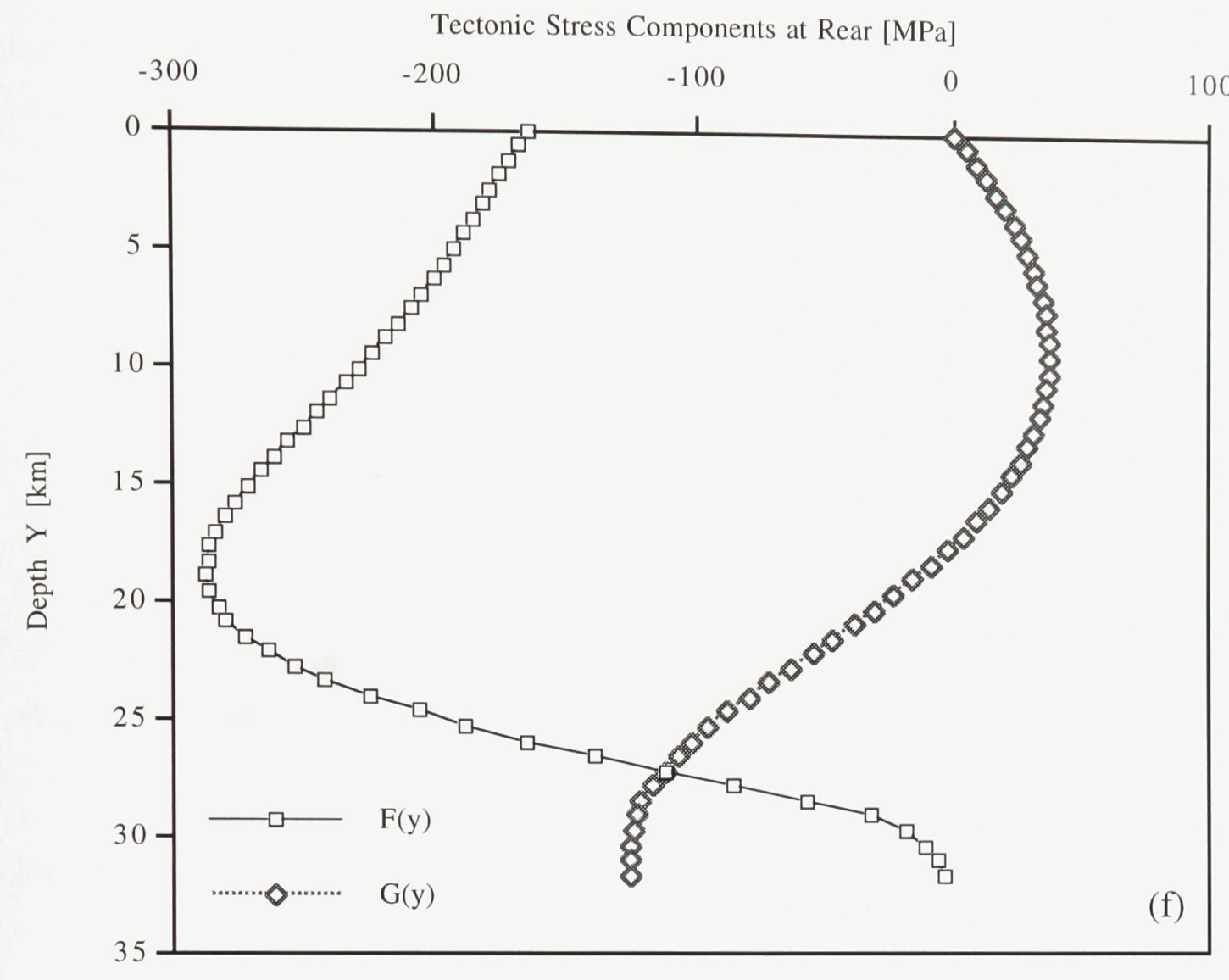

Figure 6.11 (f) tectonic-induced normal and shear stresses along the rear boundary of the wedge. 
Table 6.4 Parameters for an anorthosite wedge ${ }^{\dagger}$ with $L=200 \mathrm{~km}, \alpha=3^{\circ}$ and $\beta=6^{\circ}$, subject to a geothermal gradient of $15 \mathrm{~K} \mathrm{~km}^{-1}$.

\begin{tabular}{|c|c|c|c|c|}
\hline & \multirow[b]{2}{*}{ Parameter $\ddagger$} & \multicolumn{3}{|c|}{ Case* } \\
\hline & & A3 & B3 & $\mathrm{C} 3$ \\
\hline & $u_{0}\left(\times 10^{-10} \mathrm{~m} \mathrm{~s}^{-1}\right)$ & 0 & 0 & 0 \\
\hline & $u_{1}\left(\times 10^{-15} \mathrm{~s}^{-1}\right)$ & 1 & -1 & 1 \\
\hline \multirow{2}{*}{-} & $u_{2}\left(\times 10^{-20} \mathrm{~m}^{-1} \mathrm{~s}^{-1}\right)$ & -0.225 & -2.25 & -1 \\
\hline & \multicolumn{4}{|c|}{$\begin{array}{l}\text { * Cases A3, B3, and } \mathrm{C} 3 \text { refer to Figures } 6.12,6.13 \text {, and } 6.14 \\
\text { A3: Extensional wedge } \\
\text { B3: Compressional wedge } \\
\text { C3: Wedge with extension at the rear and compression at the front } \\
\dagger \quad \text { rheological parameters for anorthosite: } A_{0}=2.06 \times 10^{-23}\left(\mathrm{~Pa}^{-3.2} \mathrm{~s}^{-1}\right), n=3.2, Q=238\left(\mathrm{~kJ} \mathrm{~mol}^{-1}\right)\end{array}$} \\
\hline
\end{tabular}

\subsubsection{Extensional wedge}

The whole wedge in case A3 (Figure 6.12) is in extension with decreasing longitudinal extension from $10^{-15} \mathrm{~s}^{-1}$ at the rear to $10^{-16} \mathrm{~s}^{-1}$ at the front of the wedge. The predominant flow direction is from the rear towards the front of the wedge, with maximum velocity magnitude at the rear lower power-law corner region (Figure 6.12a). The trajectories of the maximum shear stress (Figure 6.12b) show a series of potential normal faults concave upward and dipping towards the front. The effective shear strain rate $\dot{\varepsilon}_{E}^{\prime}$ (Figure 6.12c) varies mainly with depth with most of the deformation concentrated at the rear lower power-law region. The effective shear stress $\sigma_{E}^{\prime}$ (Figure $6.12 \mathrm{~d}$ ) varies mainly in the $y$-direction (the small staircase variations are introduced by the computation algorithm as explained in the context of case A2). The effective viscosity (Figure 6.12e) decreases significantly within the rear lower power-law region. The nongravitational normal stress at the rear of the wedge required to maintain the deformation regime is extensional (Figure 6.12f), increasing with depth in the top plastic 


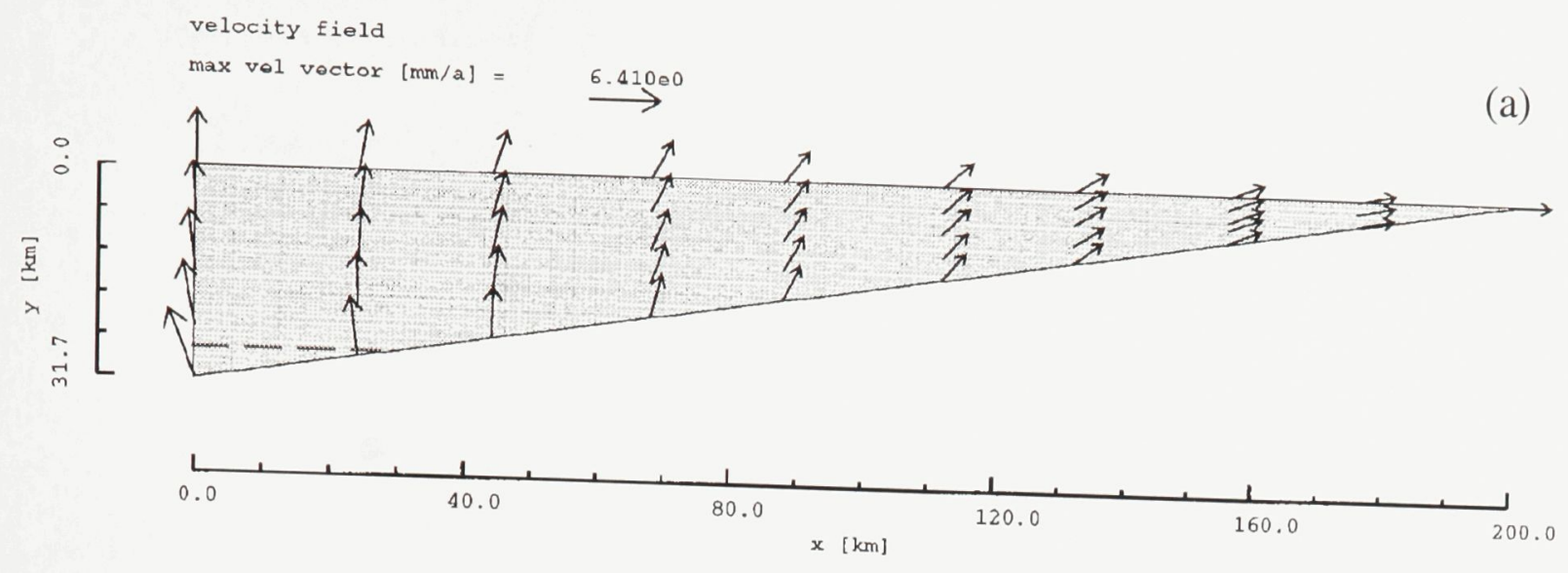

Potential Faulting Trajectories

Internal Friction Angle [deg] $=45$

(b)

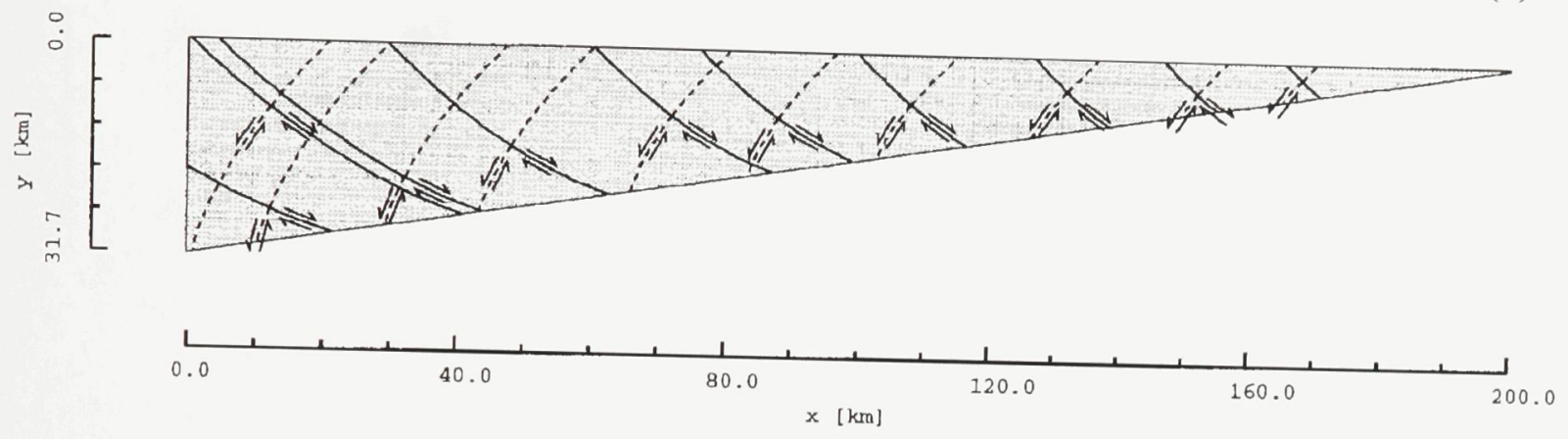

second invar. strain rate, $[1 / s]$

(c)
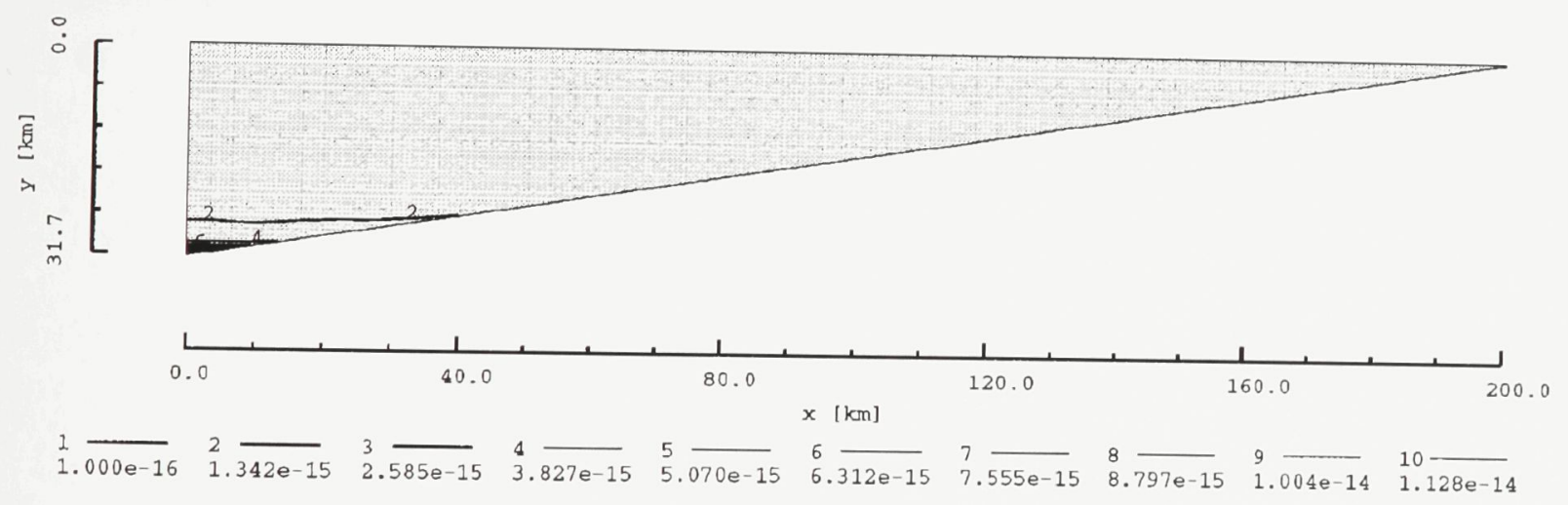

Figure 6.12 Stress and deformation within the wedge for case A3 of Table 6.4. (a) Velocity field; (b) maximum shear stress trajectories; (c) second invariant of strain rate tensor;

(continued) 

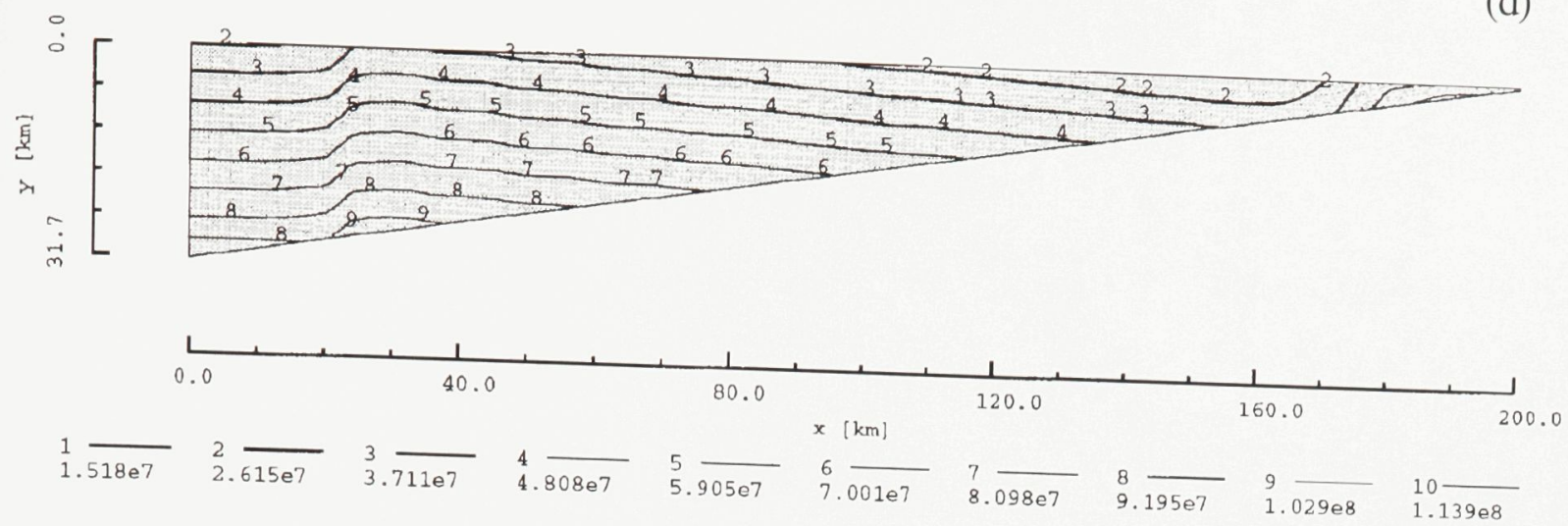

Log10[eff viscosity] [Pa s]

(e)
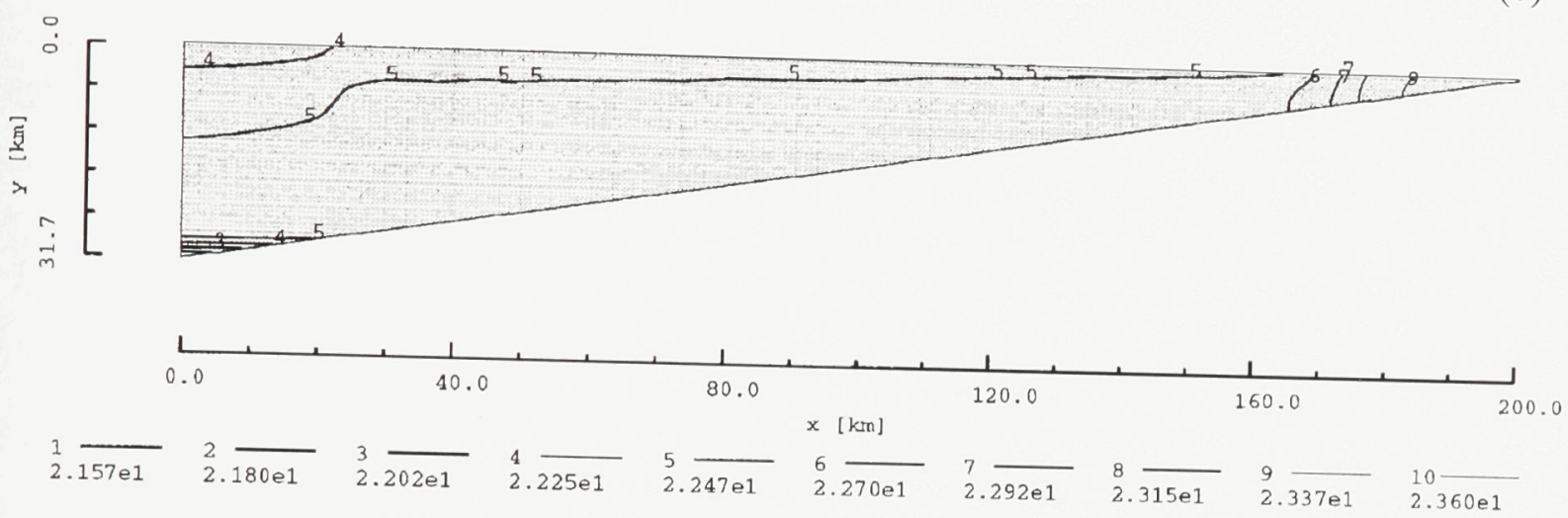

Figure 6.12

d) second invariant of deviatoric stress tensor; (e) effective viscosity;

(continued) 


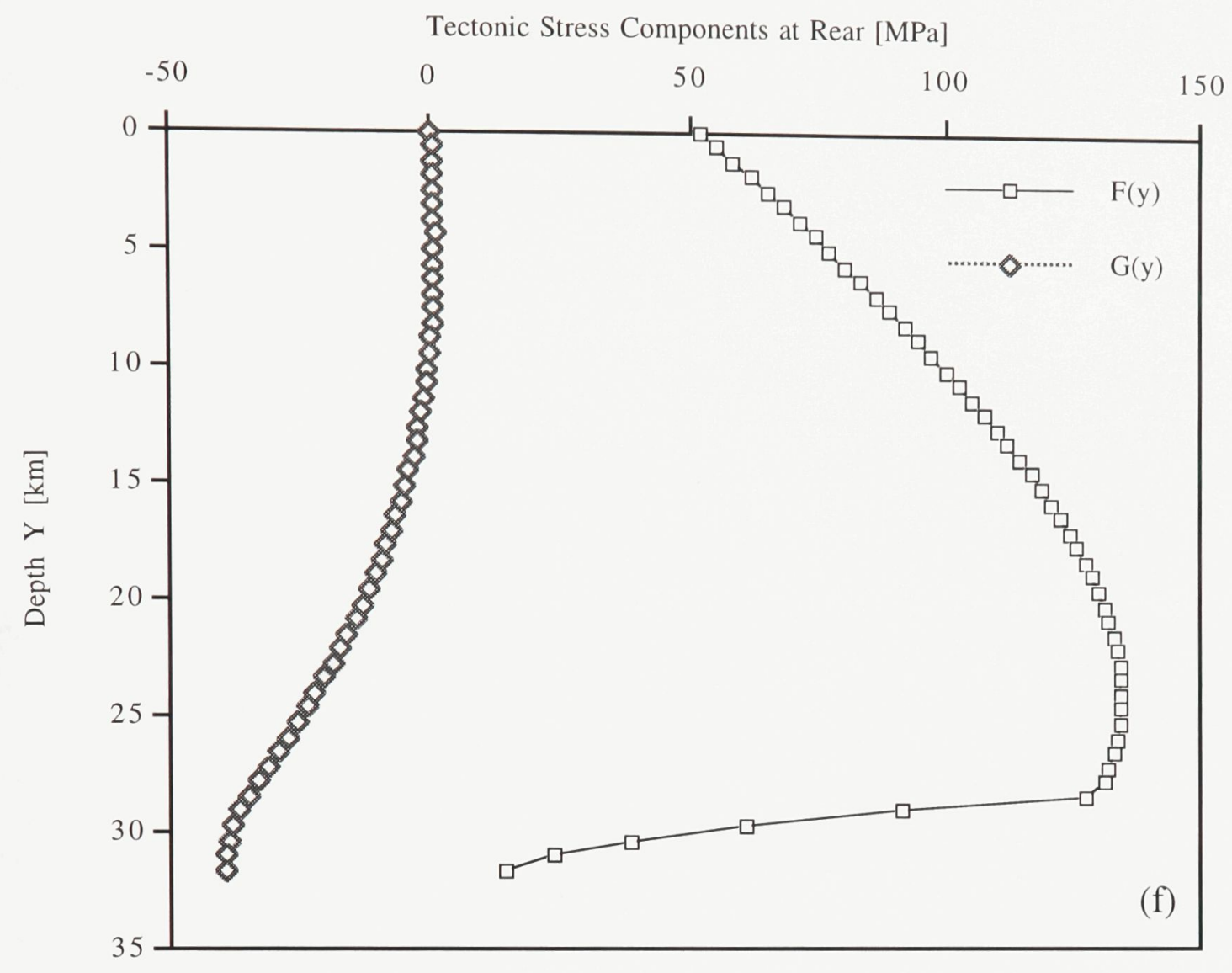

Figure 6.12 (f) tectonic-induced normal and shear stresses along the rear boundary of the wedge. 
layer and then rapidly decreasing; the nongravitational shear stress at the rear of the wedge increases nonlinearly with depth, reaching a maximum magnitude of about -40 $\mathrm{MPa}$ at the bottom of the wedge.

\subsubsection{Compressional wedge}

The whole wedge in case B3 (Figure 6.13) is in compression with increasing longitudinal compression from $-10^{-15} \mathrm{~s}^{-1}$ at the rear to $-10^{-14} \mathrm{~s}^{-1}$ at the front of the wedge. The longitudinal component of the velocity decreases gradually from about -3.5 $\mathrm{cm} \mathrm{a}^{-1}$ at the front to zero at the rear while the vertical component increases from zero at the front to $-0.5 \mathrm{~cm} \mathrm{a}^{-1}$ at the rear (Figure 6.13a). The maximum shear stress trajectories (Figure $6.13 \mathrm{~b}$ ) indicate a compressive deformation regime throughout the wedge, represented by a series of upwardly concave thrusts dipping towards the hinterland of the wedge. The effective shear strain rate $\dot{\varepsilon}_{E}^{\prime}$ (Figure $6.13 \mathrm{c}$ ) varies mainly in the longitudinal direction within the upper plastic layer, increasing from $10^{-15} \mathrm{~s}^{-1}$ at the rear to $10^{-14} \mathrm{~s}^{-1}$ at the front of the wedge; $\dot{\varepsilon}_{E}^{\prime}$ becomes an increasing function of depth only within the rear lower power-law region. The effective shear stress $\sigma_{E}^{\prime}$ (Figure 6.13d) varies mainly in the $y$-direction. The effective viscosity $\eta$ (Figure 6.13e) varies in both the $x$ - and the $y$ directions in the top plastic layer, increasing with depth and decreasing from the rear towards the front of the wedge; $\eta$ decreases with depth within the rear lower power-law region. The nongravitational normal stress at the rear of the wedge is compressional (Figure 6.13f), increasing within the top plastic layer and decreasing rapidly in the nonlinearly viscous layer; the nongravitational shear stress at the rear of the wedge varies nonlinearly with depth. 


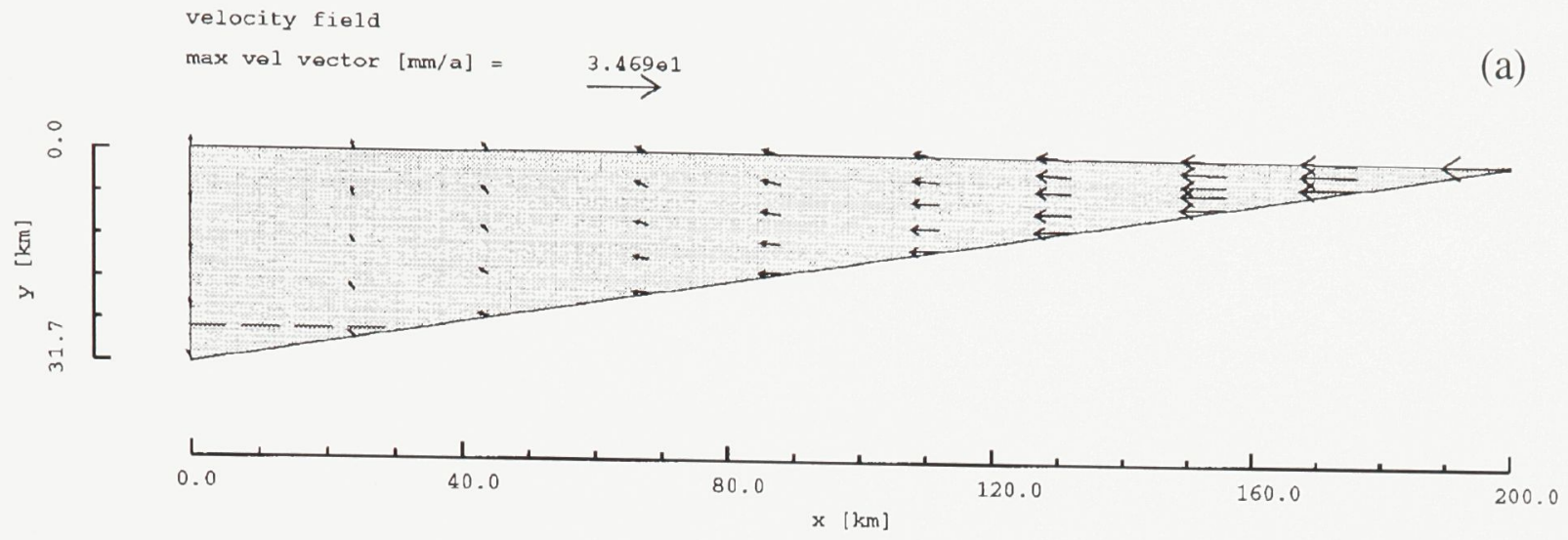

Potential Faulting Trajectories

Internal Friction Angle [deg] $=45$

(b)
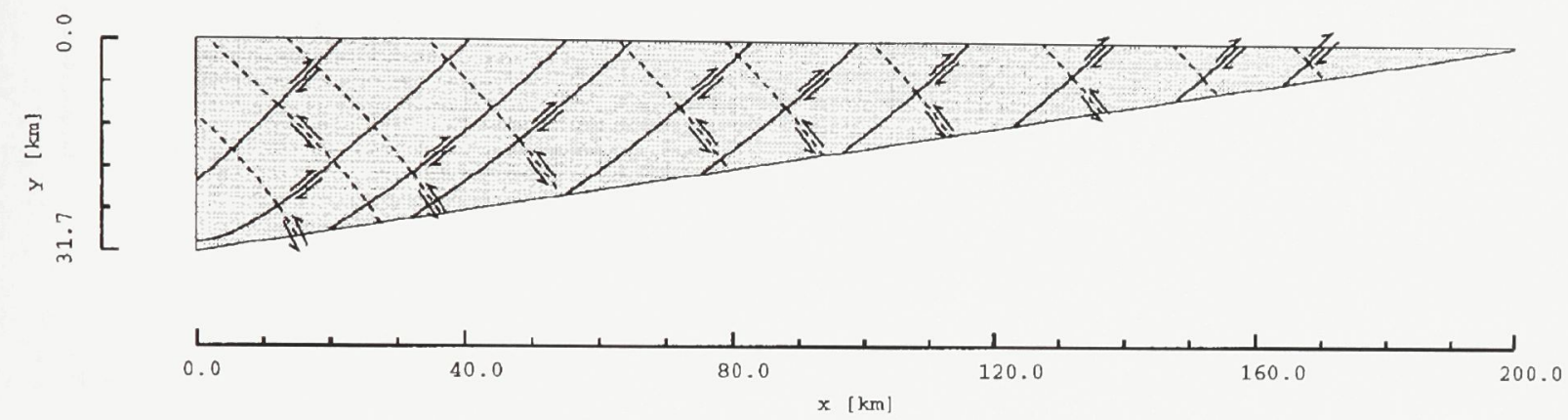

second invar. strain rate, [1/s]

(c)
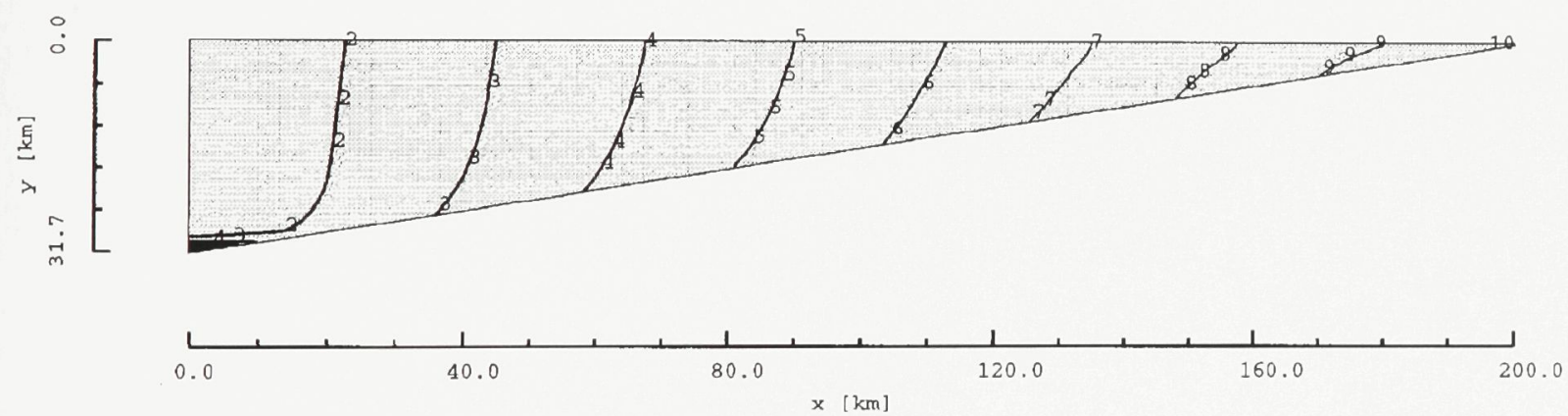

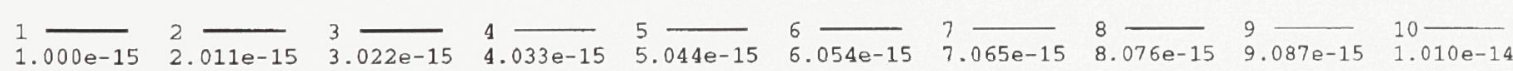

Figure 6.13 Stress and deformation within the wedge for case B3 of Table 6.4. (a) Velocity field; (b) maximum shear stress trajectories; (c) second invariant of strain rate tensor; (continued) 

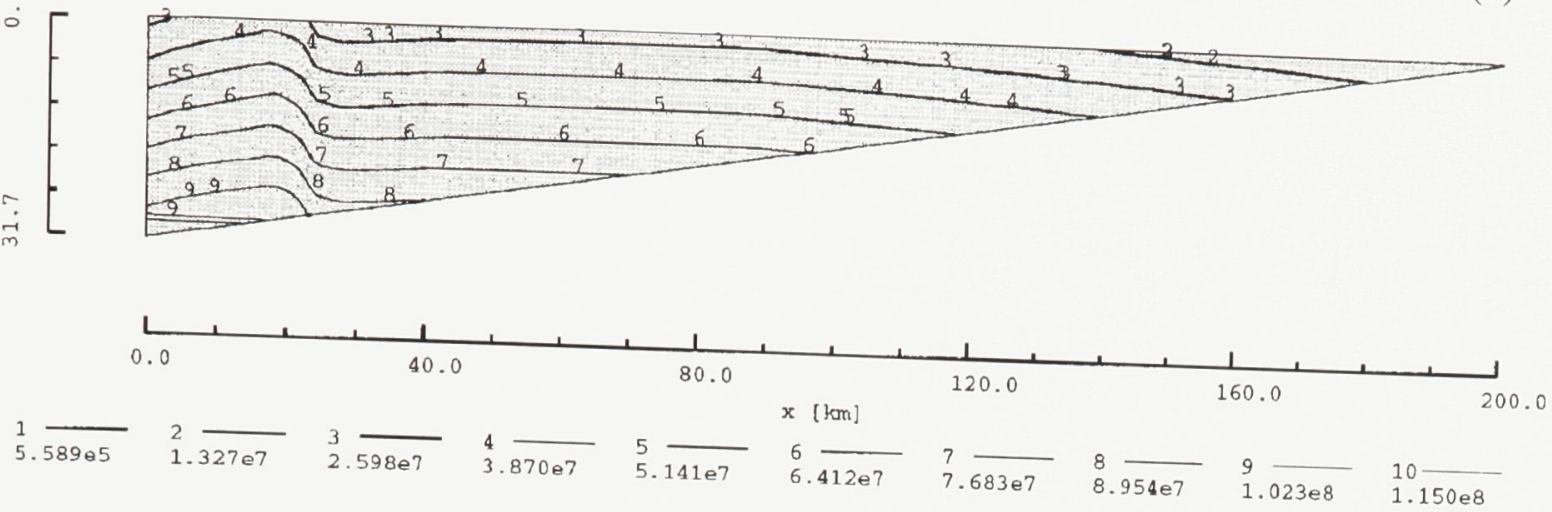

Log10[eff viscosity] [Pa s]
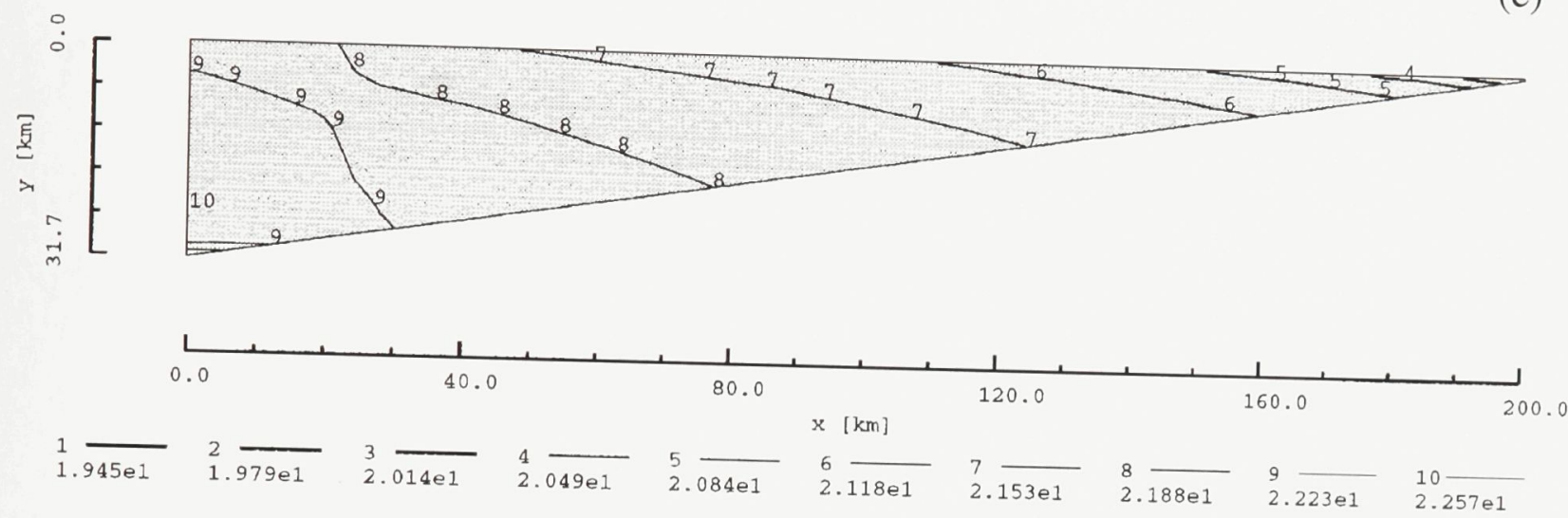

Figure 6.13 (d) second invariant of deviatoric stress tensor; (e) effective viscosity;

(continued) 


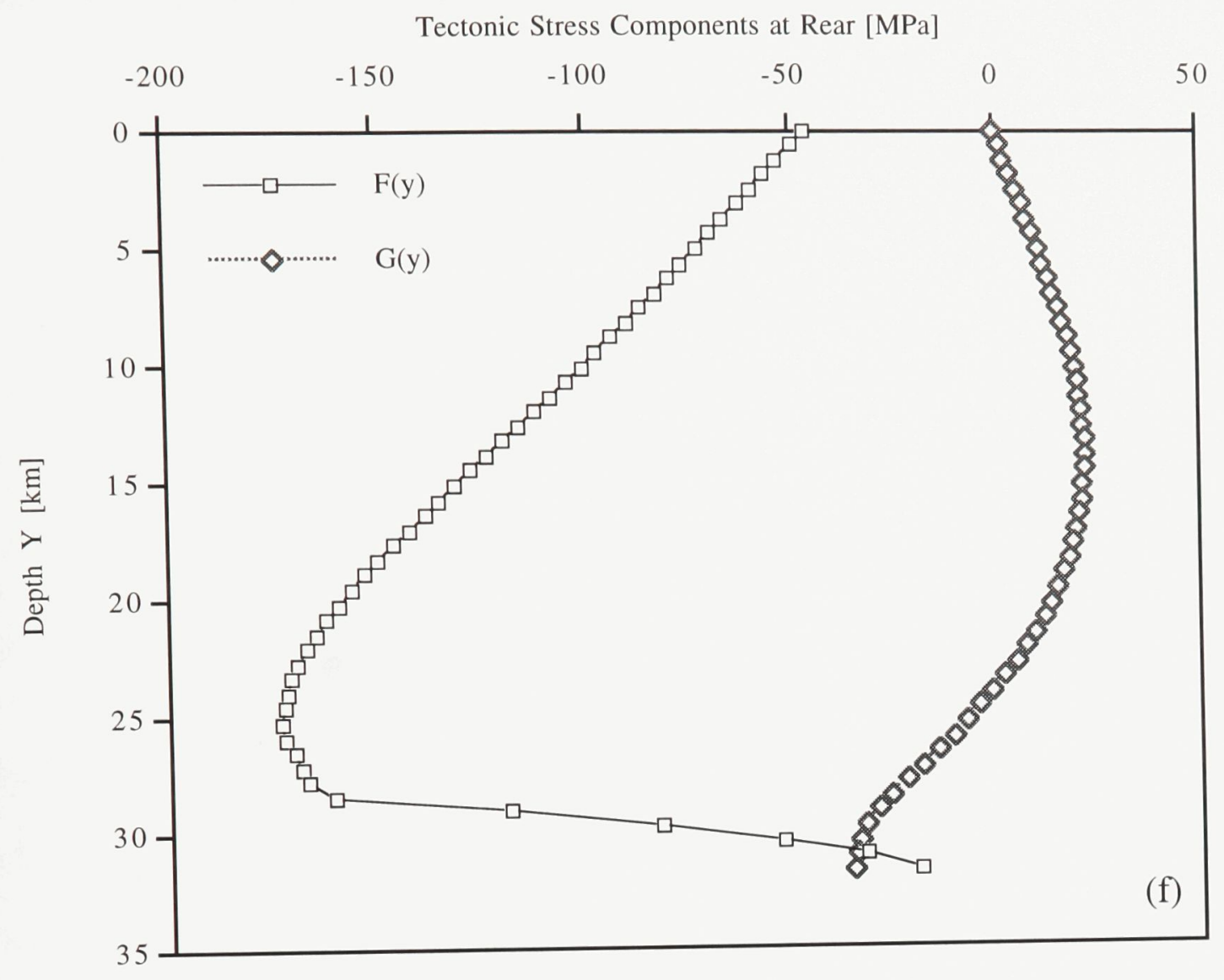

Figure 6.13 (f) tectonic-induced normal and shear stresses along the rear boundary of the wedge. 


\subsubsection{Wedge with rear extension and frontal compression}

The wedge in case C3 (Figure 6.14) is given a velocity boundary condition resulting in a longitudinal deformation pattern which is positive (extensional) at the rear and negative (compressional) at the front of the wedge. Changes of the velocity field within the wedge are much more significant in direction than in magnitude (Figure 6.14a): the movement of wedge material at the front is basically longitudinal while that at the rear becomes mainly vertical. The maximum shear stress trajectories (Figure 6.14b) reflect the existence of a compressional and an extensional deformation regime at the front and at the rear of the wedge, respectively. The effective shear strain rate $\dot{\varepsilon}_{E}^{\prime}$ (Figure 6.14c) is highly concentrated in the rear lower power-law region. The effective shear stress $\sigma_{E}^{\prime}$ (Figure 6.14d) varies mainly in the $y$-direction. The effective viscosity $\eta$ (Figure 6.14e) varies in both the $x$ - and the $y$ - directions with maximum $\eta$ (corresponding to minimum strain rate) at the boundary region separating the rear extension from the frontal compression. The nongravitational normal stress at the rear of the wedge (Figure 6.14f) is tensional, increasing with depth, as usual, in the top plastic layer and decreasing with depth in the lower viscous region. The nongravitational shear stress at the rear of the wedge varies nonlinearly with depth.

The above three cases share a low geothermal gradient. If the geothermal gradient is higher, the size of the nonlinear viscous region increases. By analogy with the cases in the previous section, a laminar flow pattern is expected to become more predominant in the lower viscous part of the wedge; the effective strain rate is also expected to be increasingly concentrated in the viscous area. 


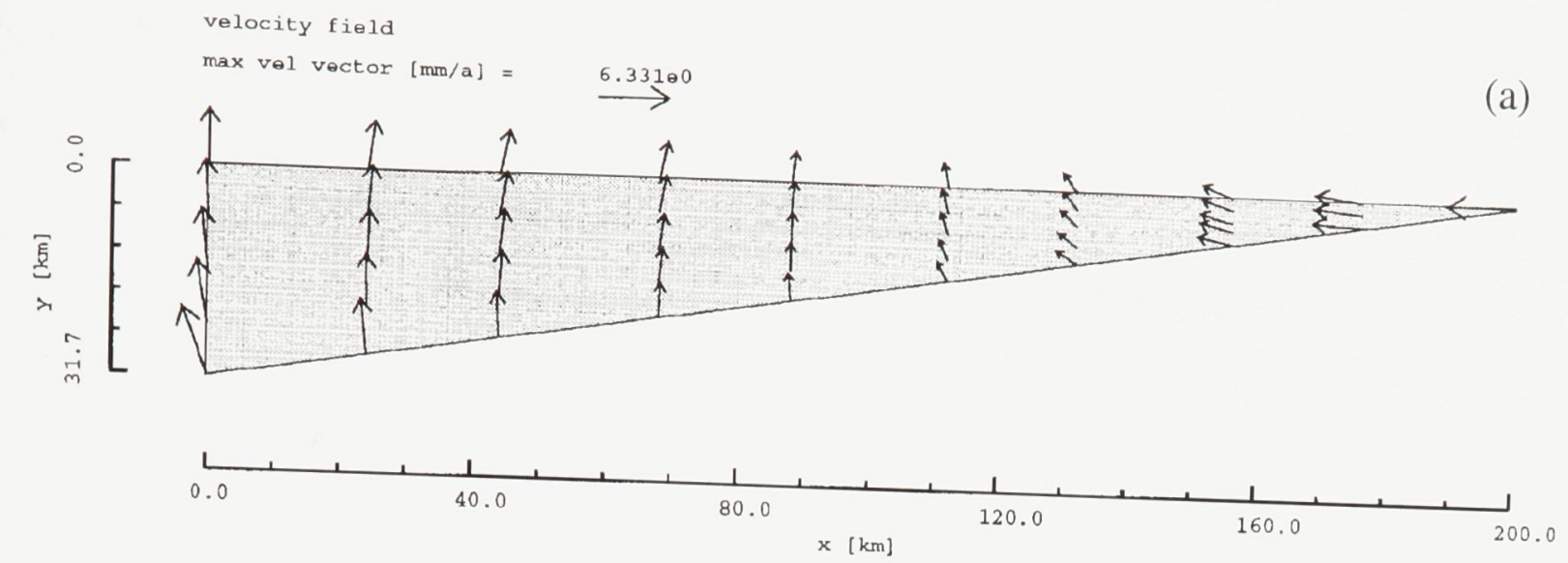

Potential Faulting Trajectories

Internal Friction Angle [deg] $=45$

(b)
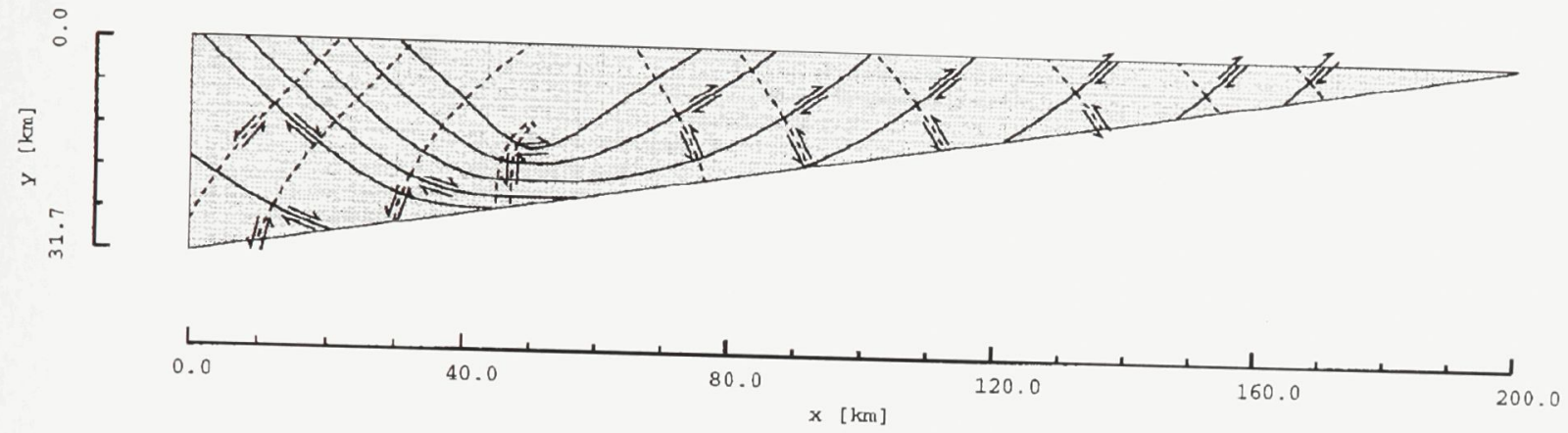

second invar. strain rate, [1/s]

(c)
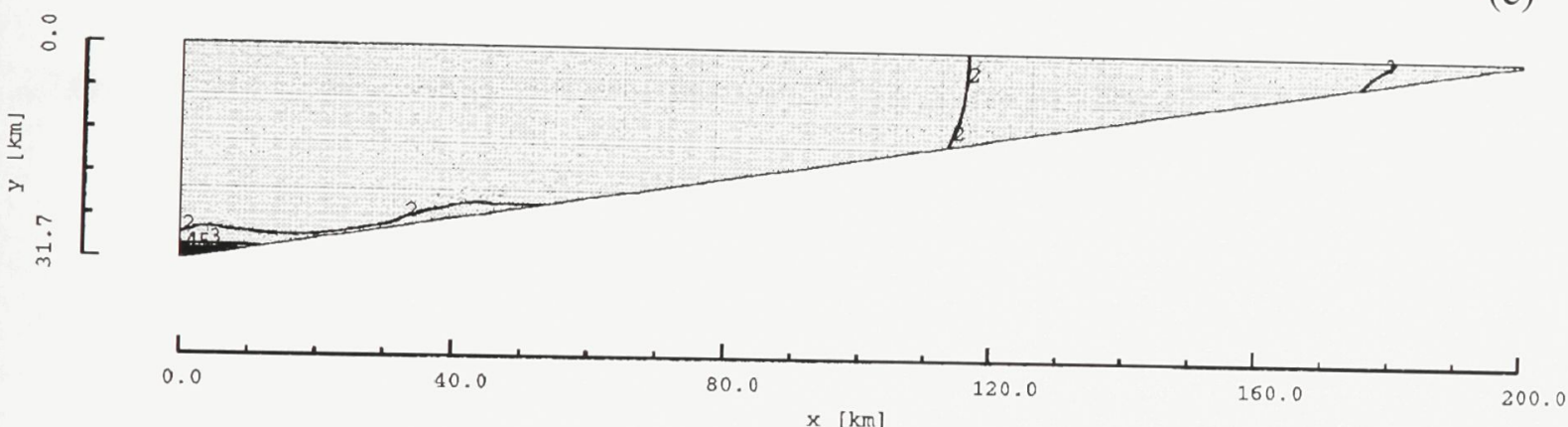

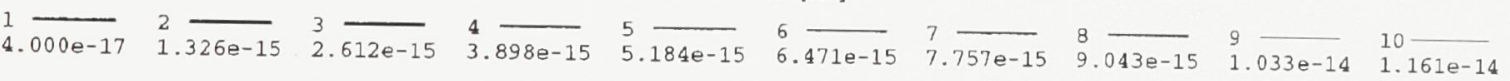

Figure 6.14 Stress and deformation within the wedge for case C3 of Table 6.4. (a) Velocity field; (b) maximum shear stress trajectories; (c) second invariant of strain rate tensor;

(continued) 

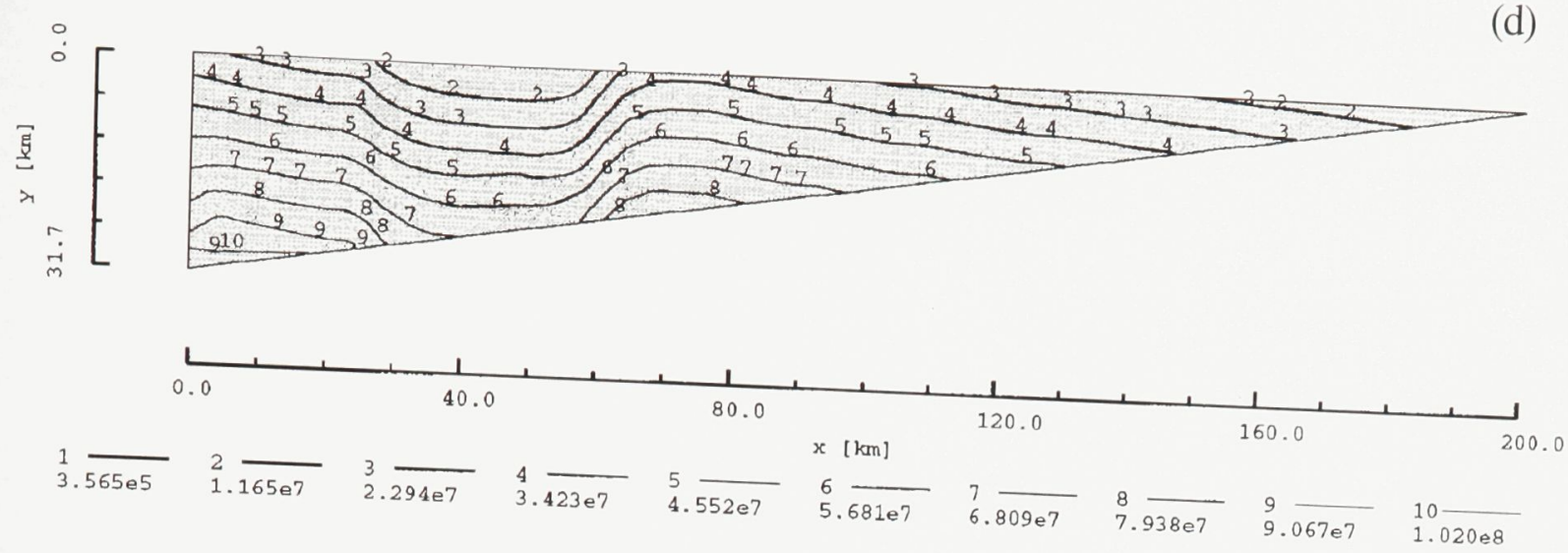

$\log 10[$ efE viscosity] [Pa 3$]$

(e)
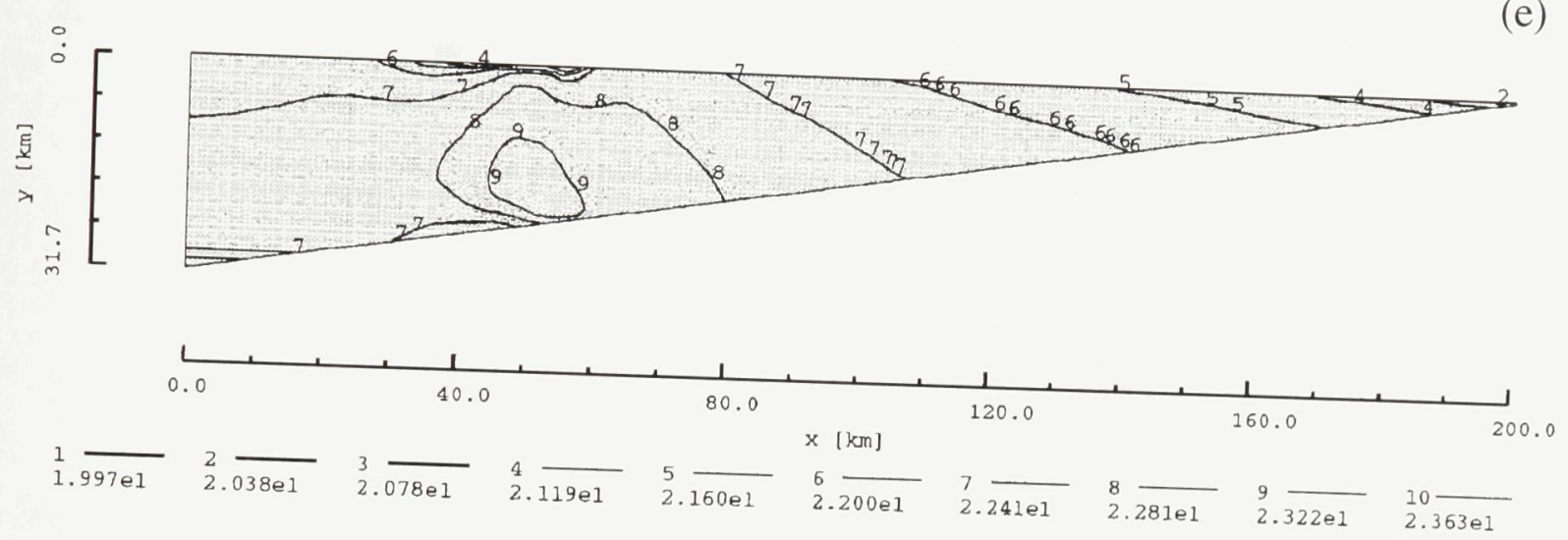

Figure 6.14

(d) second invariant of deviatoric stress tensor; (e) effective viscosity;

(continued) 


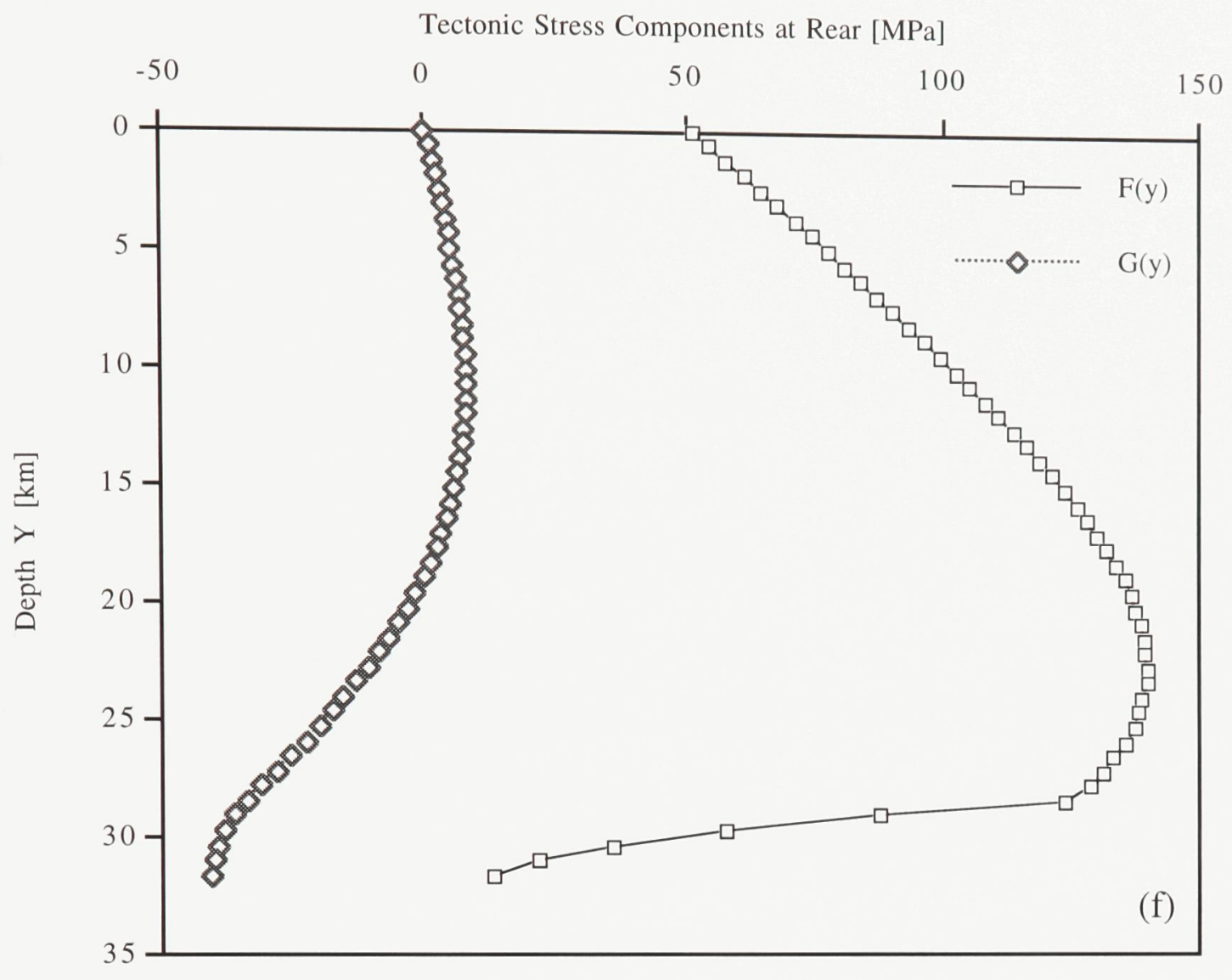

Figure 6.14 (f) tectonic-induced normal and shear stresses along the rear boundary of the wedge. 


\subsection{Some observations}

The cases studied in this chapter, as well as those in the previous chapter, show that wedges with linear, nonlinear, and a combination of rheologies, submitted to prescribed longitudinal strain rates on their top surfaces and prescribed shear stresses on their bases, can deform in compression, extension, or a combination of the two (compression in the frontal part and extension in the back). The detailed patterns of velocity, maximum shear stress trajectories, effective deviatoric stress and strain rate, and effective viscosity depend on the bulk rheology, geothermal gradient, hydration, and boundary conditions. Some solutions include relatively important vertical velocity components throughout the rear of the wedges. In nature, these could be due to underplating, with consequent exhumation of rocks (assuming that erosion keeps pace with uplift).

The conditions at the "backstop" of the wedge which result in these stress and deformation patterns are expressed in terms of the function $F(y)$, which depends mainly on bulk rheology plus the prescribed longitudinal strain rates on the top and the prescribed shear stresses on the base of the wedge (the other function $G(y)$ is related to $F(y)$.). $\quad F(y)$ represents the nongravitational horizontal normal stress on the rear boundary, i.e., the normal stress in excess of the lithostatic. As a rule, the absolute value of $F(y)$ (which is compressive in some cases and tensile in others, but never changes sign in a given case) decreases with increasing ductility (i.e., depth) for depth-dependent viscous rheology. For plastic rheology overlying a viscous layer, $F(y)$ increases in magnitude with depth within the top plastic layer, and then decreases with increasing depth in the viscous layer. These general trends are in agreement with usual measurements and analyses of stresses in the crust (cf. e.g. Ranalli, 1995), although the details of the depth variation may not be entirely corresponding to natural conditions. 


\section{Chapter 7}

\section{Conclusion}

\subsection{Main results}

(1) On the scale of an individual thrust sheet, a solution for sheer and normal stress along, the basal thrust shows that, for wedge-shaped overthrust blocks and/or nonlinear boundary conditions, static (frictional) stability conditions are exceeded only towards the use of the block. This results confirms the observation by Price $(1988,1990)$ that the solution to the paradox of large overthrusts lies in the consecutive - rather than synchronous - nature of slip (cf. chapter 3 ).

(2) On the scale of a whole orogenic wedge, an analytical solution for velocity and stress in a two-dimensional plane strain incompressible block with power-law creep rheology has been derived and explicitly expressed in terms of bulk rheology, state of stress at the rear boundary of the block, and a function $C(x, y)$. representing the deviation of the normal stress in $y$-direction from the lithostatic overburden. Although a general analytical expression for the function $C(x, y)$ is difficult to derive in the general case, such a systematic description of the dynamics of a two-dimensional block provides the necessary theoretical background on which solutions for two special cases of relevance to orogenic wedges are derived, namely,

(i) In the particular case in which the viscous rheology of the material is linear (i.e., Newtonian flow), the function $C(x, y)$ becomes determinable from the stress and/or velocity boundary conditions, making the problem analytically solvable (cf. Chapter 4);

(ii) When the top slope of the block is small and the vertical gradient of the vertical normal stress is negligible compared to the longitudinal gradient of the shear stress, the 
function $C(x, y)$ becomes zero, and a closed form for the velocity and stress solution is derived in terms of a generalized power-law creep rheology and stress/velocity boundary conditions ( $c f$. Chapter 5).

(3) The solution for power-law creep rheology and linearly varying longitudinal strain rate (case 2(ii) above) is a two-dimensional extension of a classic one-dimensional solution for ice sheets (Nye, 1957). Also, some semi-qualitative tectonic models for orogenic wedges (Platt, 1986) can be derived from the two-dimensional solution.

(4) Stress and velocity fields within orogenic wedges vary with material rheology, temperature, and boundary conditions. In all cases, computational results match reasonably well geological observables such as stress and strain rate intensities, maximum shear trajectories, etc. Orogenic wedges are deformed by the nongravitational normal stress at the rear of the wedge (i.e., $F(y)$ ), the longitudinal compressive stress due to the top slope of the wedge (i.e., $\bar{\rho} g_{x} y$ ), and the basal shear stress (i.e., $\tau_{b}$ ). Large basal shear stress favours compression and low basal shear stress favours extension within the wedge.

(5) The depth distribution of the nongravitational normal stress required to maintain the deformation regime (i.e., $F(y)$ ) varies with wedge rheology and basal shear stress. In general, it decreases with increasing ductility. In rheologically layered wedges, it increases in magnitude with depth when bulk rheology is plastic, but decreases when the rheology is power-law viscous.

(6) Wedges under appropriate stress and velocity boundary conditions are able to deform with different patterns in their longitudinal direction, that is, compression near the front and extension near the rear of the wedge ( $c f$. chapter 6). However, in order to have a normal faulting regime at the rear, the nongravitational stress $F(y)$ must be tensional. This does not preclude topography-induced extension in a compressional regime, as the present models do not take into accounts the effects of changing topography. 
(7) Although velocity boundary conditions are assigned on the surface of the wedges, rather than on its basement, computational results suggest that the uplift rates at the surface and along the basement do not vary significantly at the rear of the wedges. This implies that assigned vertical components of velocity can be taken to simulate underplating. In an analogous manner, basement-parallel components of velocity can be taken to simulate dragging by underthrusting.

\subsection{Discussion}

The nonlinear viscous solution provides a comprehensive, analytical means to represent the stress and deformation of an orogenic wedge composed of material lacking a long-term yield strength. Under any nonzero deviatoric stress, such an orogenic wedge tends to flow internally (and therefore the surface slope $\alpha$ is modified), until it reaches a stable configuration, in which the gravitational forces generated by its surface slope balance the given basal shear stress (say, exerted by the subduction). Therefore, the surface slope of the wedge should be envisioned as a dynamic variable which is adjusted throughout the whole deformation process of the wedge.

This solution can also be readily applied to investigate the exhumation of high pressure-low temperature metamorphic rocks near the rear of an unstable orogenic wedge in terms of the pressure-temperature conditions at various times (Rubie, 1984; Platt and Lister, 1985; Platt, 1993; Ranalli, 1995). Combined with a given erosion rate at the top surface of the wedge, the velocity expression of the solution (i.e., equation 5.30c) provides an estimation for the exhumation rate of the material throughout the wedge. Integration of the exhumation rate with depth leads to the time variation of the pressure conditions of rocks within the wedge. As a first approximation, the depth variation of temperature can be independently assumed, neglecting viscous shear heating. 
Consideration of viscous shear heating will lead to thermomechanical coupling, which significantly increases the complexity of the problem (for a detailed discussion, see Ranalli, 1995).

Various mechanisms for the uplift of high-pressure metamorphic rocks near the rear of an orogenic wedge have been proposed, including (a) buoyant rise (Ernst, 1984); (b) entrainment of high-pressure blocks in relatively low-density diapirs (England and Holland, 1979; Carlson, 1981; Moore, 1984); and (c) regional uplift coupled with erosion at the surface (Platt, 1975; Rubie, 1984). Computational results in this thesis indicate that uplift rates at the top and underplating rates along the basement do not differ significantly at the rear of the wedge, suggesting that erosion rate at the surface, coupled with tectonic extension, could be an essential factor in determining the P-T-t path for high-pressure metamorphic rocks in large orogenic wedges.

In order to test the applicability of the nonlinear viscous solution, computational results should be compared with relevant geological observations (such as strain rate at the surface and P-T-t paths of metamorphic rocks near the rear of the wedge), which also provide necessary boundary constraints to the dynamic modelling of each individual orogenic wedge. For instance, precise strain rate data can be obtained using Global Positioning System technology in various parts of orogenic belts (Crespi et al., 1996). Such surface strain rate data can be readily used as boundary conditions in modelling the dynamics of orogenic wedges.

There are two major problems that have to be dealt with in the study of the stress and deformation fields in orogenic wedges. The first is to derive a closed form of a general solution capable of describing orogenic wedges with complex nonlinear rheologies and complex deformation patterns which may vary in the longitudinal and the vertical directions. The second is to implement particular cases of the solution in a way suitable to the modelling of wedge deformation under acceptably realistic conditions. 
One of the basic assumptions adopted in this thesis in the derivation of the analytical solution is that the gradient of shear stress in the $x$-direction is negligible compared with the gradient of vertical normal stress in the $y$-direction, which results in $C(x, y)=0$, that is, the normal stress in $y$-direction is equal to the lithostatic overburden at any depth. This approximation may hold well in the shallower part of the wedge, but not necessarily in the rear deeper parts where deformation is often concentrated. Therefore, future studies of orogenic dynamics should include, if possible, estimation of the spatial distribution of the function $C(x, y)$ and its effect on the evolution of orogenic wedges. This pattern is of general tectonic significance, since the "zero $C(x, y)$ " assumptions has long been taken as one of the basic approximation in numerous geological and tectonic models ( $c f$. Elliott, 1976; Davis et al., 1983; Platt, 1986).

Another basic assumption in this thesis is that the longitudinal strain rate, at any depth, is a linear function of the distance from the backstop. this allows the accommodation of strain rates and theologies varying in the longitudinal direction, by segmentation of the wedge. In theory, it should be possible to accommodate nonlinearlyvarying longitudinal strain rates, which is another problem awaiting solution.

In the present analytical solution, the nongravitational stresses along the rear vertical boundary plane (i.e., $F(y)$ and $G(y)$ ) are derived quantities, i.e., those necessary to maintain a linear longitudinal strain rate under specified top and bottom boundary conditions. Ideally, they should be assigned as part of prescribed boundary conditions, but this is not possible in the framework of the present solution. Also, the velocity boundary condition should be specified on the wedge basement instead of on the top surface. Such improvements may be realized by adopting finite element method formalism, which in the present context involves a further subdivision of the wedge, not only in the longitudinal direction but also in the vertical direction. Values of $F(y)$ and 
$G(y)$ could be assigned at a number of points along the rear vertical boundary. However, implementation of such boundary conditions may prove to be difficult.

On the positive side, the analytical solution presented in this thesis has the advantage of generality. Since a large number of geological and dynamic parameters have been included, this solution can be applied to model the dynamics of different types of orogenic wedges and other geological processes. 


\section{References}

Anderson, E. M., The dynamics of faulting and dyke formation with applications to Britain, 2nd edn. Oliver \& Boyd, Edinburgh 1951

Artyushkov, E. V., Geodynamic, Elsevier, Amsterdam, 1983

Atkinson, B. K., Stress corrosion and the rate-dependent tensile failure of a fine-grained quartz rock, Tectonophyscis, 65, 281 - 290, 1980

Beaumont, C. and G. Quinlan, A geodynamic framework for interpreting crustal-scale seismic-reflectivity patterns in compressional orogens, Geophys. J. Int., 116, 754-783, 1994

Beaumont, C., P. Fullsack, and J. Hamilton, Erosional control of active compressional orogens, in McClay, K. R. (ed.) Thrust Tectonics, Chapman \& Hall, 1 -18, 1992a

Beaumont, C., P. Fullsack, J. Hamilton, and S. D. Willett, Preliminary results from a mechanical model of the tectonics of compressive crustal deformation, in Ross, G. M. (ed.) Alberta basement transects, workshop report, Volume 28, Lithoprobe Secretariat, University of British Columbia, $22-61,1992 b$

Bebout, G. E., Geometry and mechanics of fluid flow at 15 to $45 \mathrm{~km}$ depths in an early Cretaceous accretionary complex, Geophys. Res. Lett., 18, 923 -926, 1991

Behrmann, J. H., K. Brown, J. C. Moore, A. Mascle, and E. Taylor, Evolution of structures and fabrics in the Barbados accretionary prism: insights from Leg 110 of the Ocean Drilling Program, J. Struct. Geol., 10, 577 - 591, 1988

Birch, F., Role of fluid pressure in mechanics of overthrust faulting: Discussion, Geol. Soc. Am. Bull., 72, 1441-1444, 1961

Blacic, J. D., and J. M. Christie, Plasticity and hydrolytic weakening of quartz single crystals, J. Geophys. Res., 89, 4223 - 4239, 1975

Bott, M. H. P., Stress distribution and plate boundary force associated with collision mountain ranges, Tectonophysics, 182, 193 - 209, 1990 
Bott, M. H. P., Modelling the plate-driving mechanism, J. Geol. Soc., 150, 941 - 951 , 1993

Boyer, S., and D. Elliott, Thrust systems, Am. Assoc. Petrol. Geol. Bull., 66, 1196 - 1230, 1982

Brown, R. L., C. Beaumont, and S. D. Willett, Comparison of the Selkirk fan structure with mechanical models: Implications for interpretation of the southern Canadian Cordillera, Geology, 21, $1015-1018,1993$

Brown, R. L., S. D. Carr, B. J. Johnson, V. J. Coleman, F. A. Cook, and J. L. Varsek, The Monashee decollement of the southern Canadian Cordillera: a crustal-scale shear zone linking the Rocky Mountain foreland belt to lower crust beneath accreted terranes, in McClay, K. R. (ed.) Thrust Tectonics, Chapman \& Hall, 357 -364, 1992

Burchchfiel, B. C., and L. H. Royden, North-south extension within the convergent Himalaya region, Geology, 13, 679-682, 1985

Burchchfiel, B. C., Z. Chen, K. V. Hodges, Y. Liu, L. H. Royden, C. Deng, and J. Xu, The south Tibetan detachment system, Himalayan orogeny: Extension contemporaneous with and parallel to shortening in a collisional mountain belt, Geol. Soc. Am. Spec. Pap., 269, 1-41, 1992

Burg, J. P., and G. M. Chen, Tectonics and structural zonation of southern Tibet, China, Nature, 311, 219-223, 1984

Byerlee, J., Friction of rocks, Pure and Applied Geophysics, 116, 615 - 626, 1978

Carlson, C., Upwardly mobile melanges, serpentinite protrusions and transport of tectonic blocks in accretionary prisms, Geol. Soc. Am. Abs. Prog., 13, 48, 1981

Chapple, W. M., Mechanics of thin-skinned fold-and-thrust belts, Geol. Soc. Am. Bull., $89,1189-1198,1978$

Crespi, J. M., Y.-C. Chan, and M. S. Swaim, Synorogenic extension and exhumation of the Taiwan hinterland, Geology, 24, 247-250, 1996 
Dahlen, F. A., Noncohesive critical Coulomb wedges: An exact solution, J. Geophys. Res., 89, 10,125 - 10,133, 1984

Dahlen, F. A., Critical taper model of fold-and-thrust belts and accretionary wedges, Ann. Rev. Earth Planet. Sci., 18, 55-99, 1990

Dahlen, F. A. and T. D. Barr, Brittle frictional mountain building 1, Deformation and mechanical energy budget, J. Geophys. Res., 94, 3906 - 3922, 1989

Dahlen, F. A., and J. Suppe, Mechanics, growth, and erosion of mountain belts, in Clark, S. P., Jr., B. C. Burchfiel, and J. Suppe, (eds.) Processes in Continental Lithospheric Deformation, Geol. Soc. Am. Spec. Paper, 218, 161 - 208, 1988

Dahlen, F. A., J. Suppe and D. M. Davis, Mechanics of fold-and-thrust belts and accretionary wedges: cohesive Coulomb theory, J. Geophys. Res., 89, 10,087 $10,101,1984$

Dalmayrac, B., and P. Molnar, Parralel thrust and normal faulting in Peru and constraints on the state of stress, Earth Planet. Sci. Let., 55, 473 - 481, 1981

De Bremaecker, J. Cl., Thrust sheet motion and earthquake mechanisms, Earth and Planetary Sci. Lett. , 83, 159 - 166, 1987

Dechesne, R. G., and E. W. Mountjoy, Multiple decollements at deep levels of the Southern Canadian Rocky Mountain Main Ranges, Alberta and British Columbia, in Mitra, S. and G. W. Fisher (eds.) Structural geology of fold and thrust belts, Johns Hopkins University Press, Baltimore, 225 - 238, 1992

Dewey, J. F. , Extensional collapse of orogens, Tectonics, 7, 1123 - 1139, 1988

Elliott, D., The motion of thrust sheets, J. Geophys. Res., 81, 949 -963, 1976

England, P. C. and T. J. B. Holland, Archimedes and the Tauern ecologites: the role of buoyancy in the preservation of the exotic tectonic blocks, Earth Planet. Sci. Lett., 44, 287-294, 1979

Ernst, W. G., California blueschists, subduction, and the significance of tectonostratigraphic terranes, Geology, 12, 436-441, 1984 
Eshelby, J. D., Dislocation theory for geophysical application, Phil. Trans. R. Soc. London, A, 274, 331 - 338, 1973

Etheridge, M. A., Differential stress magnitudes during regional deformation and metamorphism: Upper bound imposed by tensile fracturing, Geology, 11, 231 - 234, 1983

Fisher, D. and T. Byrne, The character and distribution of mineralized fractures in the Kodiak Formation, Alaska: implications for fluid flow in an underthrust sequence, $J$. Geophys. Res., 95, 9069 -9080, 1990

Fletcher, R. C., Approximate analytical solutions for a cohesive fold-and-thrust wedge: some results for lateral variation in wedge properties and for finite wedge angle, $J$. Geophys. Res., 94, 10,347 -10,354, 1989

Fowler, C. M. R., The Solid Earth, Cambridge University Press, Cambridge, 1990

Fung, Y. C., A first course in continuum mechanics, Prentice-Hall, Englewood Cliffs, 1969

Gansser, A., Geology of the Himalaya, Wiley-Interscience, New York, 1964

Gottschalk, R. R. and J. S. Oldow, Low angle normal faults in the south-central Brooks Range fold and thrust belt, Artica Alaska, Geology, 16, 395 - 399, 1988

Hafner, W., Stress distribution and faulting, Geol. Soc. Am. Bull., 62, 373 - 398, 1951

Harrison, T. M., P. Copeland, W. S. F. Kidd, and A. Yin, Raising Tibet, Science, 255, $1663-1670,1992$

Hsü, K. J., Role of cohesive strength in the mechanics of overthrust faulting and landsliding, Geol. Soc. Am. Bull., 80, 927 - 952, 1969

Hubbert, M. K., and W. W. Rubey, Role of fluid pressure in mechanics of overthrust faulting -I, Mechanics of fluid-filled solids and its application to overthrust faulting, Geol. Soc. Am. Bull., 70, 115 - 166, 1959

Hudleston, P. J., A comparison between glacial movement and thrust sheet or nappe emplacement and associated structures, in Mitra, S. and G. W. Fisher (eds.) 
Structural geology of fold and thrust belts, Johns Hopkins University Press, Baltimore, 81 - 91, 1992

Hyndman, R. D. and K. Wang, Thermal constraints on the zone of major thrust earthquake failure: the Cascadia Subduction Zone, J. Geophys. Res., 98, 2039 - 2060, 1993

Jaeger, J. C. and N. G. W. Cook, Fundamentals of rock mechanics, 3rd edn., Methuen, London, 1969

Kirby, S., H., Rheology of the lithosphere, Rev. Geophys. Space Phys, 21, 1458 - 1487, 1983

Kreyszig, E., Advanced Engineering Mathematics, John Wiley \& Sons, 1988

Liu, J. Y. and G. Ranalli, Stress in an overthrust sheet and propagation of thrusting: An Airy stress function solution, Tectonics, 11, 549 - 559, 1992

Liu, J. Y. and G. Ranalli, A 2-dimensional power-law rheology model for subductionaccretionary orogenic complexes (Abstract), EOS , 75, 598, 1994

Lliboutry, L. A., Very Slow Flows of Solids, Martinus Nijhoff Dordrecht, 1987

Lyon-Caen, H., and P. Molnar, Contraints on the structure of the Himalaya from an analysis of gravity anomalies and a flexural model of the lithosphere, J. Geophys. Res., 88, 8171-8191, 1983

Malavieille, J., Modélisation expérimentale des chevauchementss imbriqués: Application aux chaînes de montagnes, Société Géologique de France, Bulletin, 18, Ser. 7, 129 138,1984

Mandl, G. and G.K. Shippam, Mechanical model of thrust sheet gliding and imbrication, in McClay, K.R. and N.J. Price (eds.) Thrust and nappe tectonics, Geol. Soc. London, Spec. Publ. 9, 79 - 98, 1981

Mandl, G., Mechanics of tectonic faulting, Elsevier, Amsterdam, 1988

McGarr, A., and N. C. Gay, State of stress in the Earth's crust, Ann. Rev. Earth Planet. Sci.,. 6, 405 - 436, 1978 
McClay, K. R., Thrust tectonics: an introduction, in McClay, K. R. (ed.) Thrust tectonics, Chapman \& Hall, London, 41 - 52, 1992

Molnar, P. and H. Lyon-Caen, Some simple physical aspects of the support, structure, and evolution of mountain belts, Geol. Soc. Am. Spec. Pap., 31, 357 - 396, 1988

Moore, D., Metamorphic history of a high-grade blueschist exotic block from the Franciscan Complex, California, J. Petro., 25, 126-150, 1984

Moore, J. C., and P. Vrolijk, Fluids in accretionary prisms, Rev. Geophys., 30, 113 - 135, 1992

Nádai, A., Plasticity, McGraw-Hill, New York, 1931

Nye, J. F., The flow of glaciers and ice-sheets as a problem in plasticity, Proc. Roy. Soc. London, A, 207, 554 - 572, 1951

Nye, J. F., The distribution of stress and velocity in glaciers and ice sheets, Proc. Roy. Soc. London, A, 239, $113-133,1957$

Oldow, J. S., B. D’Argenio, L. Ferranti, G. Pappone, E. Marsella, and M. Sacchi, Largescale longitudinal extension in the southern Apennines contractional belt, Italy, $J$. Geology, 21, $1123-1126,1993$

Paterson, W. S. B., The physics of glaciers, 2nd ed., Pergamon Press, London, 1981

Pavlis, T. L., Origin and age of the Border Ranges fault of Southern Alaska and its bearing on the Mesozic tectonic evolution of Alaska, Tectonics, 1, 343 - 368, 1982

Pavlis, T. L. and R. L. Bruhn, Deep-seated flow as a mechanism for the uplift of broad forearc ridges and its role in the exposure of high $\mathrm{P} / \mathrm{T}$ metamorphic terranes, Tectonics, $2,473-497,1983$

Pfiffner, O. A. and J. G. Ramsay, Constraints on geological strain rates: Arguments from finite strain states of naturally, deformed rocks, J. Geophys. Res., 87, $311-321,1982$

Platt, J. P., Metamorphic and deformation process in the Franciscan Complex, California: some insights from the Catalina schist terrane, Geol. Soc. Am. Bull., $86,1337-1347,1975$ 
Platt, J. P., Dynamics of orogenic wedges and the uplift of high-pressure metamorphic rocks, Geol. Soc. Am. Bull., 97, 1037 - 1053, 1986

Platt, J. P., The uplift of high-pressure--low-temperature metamorphic rocks, Phil. Trans. R. Soc. Lond., A.321, 87 - 103, 1987

Platt, J. P., Exhumation of high-pressure rocks, Terra Nova, 5, 119 - 133, 1993

Platt, J. P. and G. S. Lister, Structural history of high-pressure metamorphic rocks in the Vanoise massif, French Alps, and their relation to Alpine tectonic events, $J$. Struct. Geol., 7, 19-36, 1985

Polino, R., G. V. Dal Piaz, and G. Gosso, Tectonic erosion at the Adria margin and accretionary processes for the Cretaceous orogeny of the Alps, Mém. Soc. Géol. Fr.ance, $156,345-367,1990$

Price, N. J., and J. W. Cosgrove, Analysis of geological structures, Cambridge Univeristy Press, Cambridge, 1990

Price, R. A., Large-scale gravitational flow of supracrustal rocks, southern Canadian Rockies, in De Jong, K. A. and R. Scholten (eds.) Gravity and tectonics, Wiley, New York, $491-502,1973 a$

Price, R. A., The mechanical paradox of large overthrusts, Geol. Soc. Am., Abst. Programs, 5, 772, 1973 b

Price, R. A., The Cordilleran foreland thrust-and-fold belt in the Southern Canadian Rocky Mountains, in McClay, K. J. and N. J. Price (eds.) Thrust and nappes tectonics, Geol. Soc. London, Spec. Publ., 9, 427 - 448, 1981

Price, R. A., The mechanical paradox of large overthrusts, Geol. Soc. Am. Bull., 100, $1898-1908,1988$

Price, R. A., The mechanical paradox of large overthrusts: reply, Geol. Soc. Am. Bull., $102,531-532,1990$

Press, W. H., S. A. Teukolsky, W. T. Vetterling, and B. P. Flannery, Numerical recipes, 2nd ed., Cambridge University Press, Cambridge, 1992 
Ranalli, G. (ed.), Heat flow, rock mechanics, and seismicity, Tectonophysics, 217, 1 115,1993

Ranalli, G., Rheology of the earth, 2nd ed., Chapman \& Hall, London, 1995

Ranalli, G. and D. C. Murphy, Rheological stratification of the lithosphere, Tectonophysics, $132,281-295,1987$

Reade, T. M., The mechanics of overthrusts, Geol. Mag., 5, 518, 1908

Reiner, M., Lectures on theoretical rheology, 3rd ed., North-Holland, Amsterdam, 1960

Royden, L. H., The Steady state thermal structure of eroding orogenic belts and accretionary prisms, J. Geophys. Res., 98, 4,487 - 4,507, 1993

Rubie, D. C., A thermal-tectonic model for high-pressure metamorphism and deformation in the Sesia zone, western Alps, J. Geol., 92, 21-36, 1984

Rutter, E. H., The kinematics of rock deformation by pressure solution, Roy. Soc. London Phil. Trans., 283A, $203-219,1976$

Rutter, E. H., Pressure solution in nature, theory and experiment, J. Geol. Soc. London, 140, $725-740,1983$

Savage, J. C., Dislocations in seismology, in Nabarro, F. R. N. (ed.) Dislocations in solids, V. 3, North-Holland, Amsterdam, 1980

Smoluchowski, M. S., Some remarks on the mechanics of overthrusts, Geol. Magazine, 6, $204-205,1909$

Spiegel, M. R., Schaum's outline of theory and problems of advanced mathematics for engineers and scientists, McGraw-Hill, New York, 1971

Suppe, J., A retrodeformable cross section of northern Taiwan, Geol. Soc. China Proc., 23, $46-55,1980$

Suppe, J., Principles of structural geology, Prentice-Hall, Englewood Cliffs, 1985

Timoshenko, S. P. and J. N. Goodier, Theory of elasticity, 3rd ed, McGraw-Hill, New York, 1970

Turcotte, D. and G. Schubert, Geodynamics, John Wiley \& Sons, New York, 1982 
Twiss, R. J. and E. M. Moores, Structural Geology, W. H. Freeman and Company, New York, 1992

von Huene, R., Tectonic processes along the front of modern convergent margins -research of the past decade, Rev. Earth Planet. Sci., 351-381, 1984

Vrolijk, P. J., Tectonic driven fluid flow in the Kodiak accretionary complex, Alaska, Geology, 15, $466-469,1987$

Washington, P. A., The mechanical paradox of large overthrusts: Alternative interpretation, Geol. Soc. Am. Bull., 102, 529 - 530, 1990

Westbrook, G. K., The Barbados Ridge complex: tectonics of a mature forearc system, in Legett, J. K. (ed.) Trench-forearc geology: sedimentation and tectonics on modern and ancient active plate margins, Geol. Soc. London, 275 - 290, 1982

Willett, S., Dynamic and kinematic growth and change of a Coulomb wedge, in McClay, K. R. (ed.) Thrust tectonics, Chapman \& Hall, London, 19 - 31, 1992

Willett, S., Beaumont, C., and P. Fullsack, Mechanical model for the tectonics of doubly vergent compressional orogens, Geology, 21, 371 -374, 1993

Wojtal, S., One-dimensional models for plane and non-plane power-law flow in shortening and elongating thrust zones, in McClay, K. R. (ed.) Thrust tectonics, Chapman \& Hall, London, 41 - 52, 1992

Woodward, N. B., Geological applicability of critical-wedge thrust-belt models, Geol. Soc. Am. Bull., 99, 827 - 832, 1987

Xiao, H. B., F. A. Dahlen, and J. Suppe, Mechanics of extensional wedges, J. Geophys. Res., 96, 10,301 - 10,318, 1991

Yin, A., A mechanical model for a wedge-shaped thrust sheet (Abstract), EOS, 67, 1242, 1986

Yin, A., Geometry, kinematics, and a mechanical analysis of a strip of the Lewis allochthon from Peril Peak to Bison Mountain, Glacier National Park, Montana, Ph.D. thesis, Univ. of S. Calif. Los Aneles, 1988 
Yin, A., Origin of regional, rooted low-angle normal faults: a mechanical model and its implications, Tectonics, 8, 469 - 482, 1989

Yin, A., Mechanics of wedge-shaped fault blocks 1, an elastic solution for compressional wedges, J. Geophys. Res., 98, 14,245 - 14,256, 1993

Zhao, W. L., D. M. Davis, F. A. Dahlen and J. Suppe, Origin of convex accretionary wedges: evidence from Barbados, J. Geophys. Res., 91, 10,246 - 10,258, 1986 